\title{
Methyl-shifted farnesyldiphosphate derivatives are substrates for sesquiterpene cyclases
}

Vanessa Harms, Benjamin Schröder, Clara Oberhauser, Cong Duc Tran, Sven Winkler,

Gerald Dräger and Andreas Kirschning*

1. Materials and methods

1.1 General information

1.2 Genes, strains, plasmids, expression and growth conditions

1.3 Protein purification

1.4 Analytical enzyme tests

\section{Chemical Syntheses}

2.1 Synthesis of tris(tetra- $n$-butylammonium)hydrogenpyrophosphate trihydrate (S1) and general description of diphosphate synthesis via the chlorides

2.2 Synthesis of farnesylpyrophosphate derivative $\mathbf{9}$

2.3 Synthesis of farnesylpyrophosphate derivative $\mathbf{1 0}$

2.4 Synthesis of farnesylpyrophosphate derivative $\mathbf{1 1}$

2.5 Synthesis of farnesylpyrophosphate derivative $\mathbf{1 2}$

\section{Optimisation of biotransformations with FPP as substrate}

3.1 Temperature and $\mathrm{pH}$

3.2 Concentration of enzyme, substrate and $\mathrm{Mg}$

3.4 Non-natural substrates

4. Biotransformations of Bot2 with unnatural farnesylpyrophosphates

4.1 General procedure for the enzymatic conversion of Bot2 with $\mathbf{9 , 1 1}$ and $\mathbf{1 2}$

4.1.1 Defined parameters

4.2 Structure elucidation of $\mathbf{2 5}$

4.3 Structure elucidation of $\mathbf{2 6}$ and $\mathbf{2 7}$

4.4 Structure elucidation of $\mathbf{2 8}$

4.4.1 Energetic calculations for $\mathbf{2 8}$

4.4.2 Structure elucidation of thermic product $\mathbf{3 1}$

\section{References (supporting information)}

\section{Attachments}

A. GC chromatograms and MS spectra

B. Copies of ${ }^{1} \mathrm{H}$ - and ${ }^{13} \mathrm{C}-\mathrm{NMR}$ spectra (chemical synthesis)

Note: Copies of NMR spectra of synthetic compounds are found in the attachments, while NMR-

spectra of new terpenoids are embedded in the main SI text. 


\section{Materials and methods}

\subsection{General information}

${ }^{1} \mathrm{H}$ NMR spectra were recorded at $400 \mathrm{MHz}$ with a BRUKER Avance- 400 and BRUKER Ascend-400 as well as at $500 \mathrm{MHz}$ with a BRUKER DRX-500 spectrometer or at $600 \mathrm{MHz}$ with an BRUKER Ascend600 or BRUKER Avance III-600 at $298 \mathrm{~K}$ or $255 \mathrm{~K}$ for low temperature NMR measurements. ${ }^{13} \mathrm{C}$ NMR spectra were recorded at $100 \mathrm{MHz}$ with a BRUKER Avance-400 and BRUKER Ascend-400 or at 125 MHz with a BRUKER DRX-500 or at $151 \mathrm{MHz}$ with a BRUKER Ascend-600/ Avance III-600 instrument. ${ }^{31} \mathrm{P}$ NMR spectra were recorded at $162 \mathrm{MHz}$ with a BRUKER Avance-400 and BRUKER Ascend-400 or at $202 \mathrm{MHz}$ with a BRUKER DRX-500 instrument. Multiplicities are described using the following abbreviations: $\mathrm{s}=$ singlet, $\mathrm{d}=$ doublet, $\mathrm{t}=$ triplet, $\mathrm{q}=$ quartet, $\mathrm{sex}=$ sextet, $\mathrm{m}=$ multiplet, $\mathrm{b}=$ broad. Chemical shift values of ${ }^{1} \mathrm{H}$ and ${ }^{13} \mathrm{C}$ NMR spectra are commonly reported in ppm relative to residual solvent signal as internal standard. The multiplicities refer to the resonances in the offresonance decoupled spectra and were elucidated using phase-sensitive HSQC experiments. Mass spectra were obtained with a lockspray dual ion source in combination with a WATERS Alliance 2695 LC system, or with a type Q-TOF premier (MICROMASS) spectrometer (ESI mode) in combination with a WATERS Acquity UPLC system equipped with a WATERS Acquity UPLC BEH C18 $1.7 \mu \mathrm{m}$ (SN 01473711315545) column (solvent A: water $+0.1 \%\{\mathrm{v} / \mathrm{v}\}$ formic acid, solvent B: MeCN or $\mathrm{MeOH}$ \{given in experimental part $\}+0.1 \%\{\mathrm{v} / \mathrm{v}\}$ formic acid; flow rate $=0.4 \mathrm{~mL} / \mathrm{min}$; gradient $\{\mathrm{t}$ [min]/solvent B [\%]\}: $\{0 / 5\}\{2.5 / 95\} \quad\{6.5 / 95\} \quad\{6.6 / 5\} \quad\{8 / 5\}$; retention times $\left\{\mathrm{r}_{\mathrm{t}}\right\}$ given in the experimental part). Ion mass signals $(\mathrm{m} / \mathrm{z}$ ) are reported as values in atomic mass units.

GC-O analyses were carried out with an Agilent GC 7890B chromatograph with Gerstel CIS4 Cold Injector, coupled to a Gerstel OPD 3 sniffer. Samples were analysed on a Zebron ZB-FFAP (7KGG009-11) column (60 m x $0.25 \mathrm{~mm}$ i.d. x film thickness $0.25 \mu \mathrm{m}$ ). Carrier gas, He; flow $1.4 \mathrm{~mL} / \mathrm{min}$; injector temp.: $50^{\circ} \mathrm{C}$ to $260{ }^{\circ} \mathrm{C}$ at $12{ }^{\circ} \mathrm{C} / \mathrm{s}$; split ratio 1:5; temp. program: $50{ }^{\circ} \mathrm{C}$ to $230{ }^{\circ} \mathrm{C}$ at $8{ }^{\circ} \mathrm{C} / \mathrm{min}$ held isothermal for $22.5 \mathrm{~min}$. Transfer to Gerstel ODP 3 is made through $1 \mathrm{~m} \times 150 \mu \mathrm{m}$ column without stationary phase at $280^{\circ} \mathrm{C}$.

GC/MS analyses were carried out either with an HP 6890 chromatograph with Gerstel MPSII and KAS 4 injector, coupled to an HP 5973 quadrupole mass selective detector. Samples were analysed on an Optima 5 column (poly(5\%-phenyl-95\%-methylsiloxane), $30 \mathrm{~m} \times 0.32 \mathrm{~mm}$ i.d. $\times$ film thickness $0.25 \mu \mathrm{m})$. Carrier gas, He; injector temp., $60^{\circ} \mathrm{C}$ to $300^{\circ} \mathrm{C}$ at $12^{\circ} \mathrm{C} / \mathrm{min}$, splitless; temp. program: $50^{\circ} \mathrm{C}$ (isothermal $1 \mathrm{~min}$ ) to $300^{\circ} \mathrm{C}$, at $20^{\circ} \mathrm{C} / \mathrm{min}$ and held isothermal for $6 \mathrm{~min}$ at $300^{\circ} \mathrm{C}$; ion source: $\mathrm{EI}$, ionisation energy, $70 \mathrm{eV}$; EI mass spectra were acquired over the mass range of $40-500 \mathrm{amu}$. Further GC/MS analyses were carried out on with an Agilent 7890B GC with 5977B GC/MSD and Gerstel MPS Robotic XL with KAS 4C injector. Samples were analysed on an Optima 5HT column, $30 \mathrm{~m} \mathrm{x}$ $250 \mu \mathrm{m}$ i.d. $\mathrm{x}$ film thickness $0.25 \mu \mathrm{m}$ ). Carrier gas, He; injector temp., $60^{\circ} \mathrm{C}$ to $300^{\circ} \mathrm{C}$ at $12^{\circ} \mathrm{C} / \mathrm{min}$, splitless; temp. program: $50^{\circ} \mathrm{C}$ (isothermal $1 \mathrm{~min}$ ) to $300^{\circ} \mathrm{C}$, at $20^{\circ} \mathrm{C} / \mathrm{min}$ and held isothermal for 6.5 min at $300^{\circ} \mathrm{C}$; FID: $300^{\circ} \mathrm{C}, \mathrm{H}_{2}: 30 \mathrm{~mL} / \mathrm{min}, \mathrm{N}_{2}: 25 \mathrm{~mL} / \mathrm{min}$, MSD: ion source: $\mathrm{EI} 70 \mathrm{eV}, 230{ }^{\circ} \mathrm{C}$; detector: quadrupole, EI mass spectra were acquired over the mass range of 30 -650 amu.

HR-GC/MS analyses were carried out on a Waters GCT Premier mass spectrometer coupled with an Agilent 6890n GC with CTC CombiPAL sampler. Samples were analysed on an Optima 5HT column, $30 \mathrm{~m} \times 250 \mu \mathrm{m}$ i.d. $\times$ film thickness $0.25 \mu \mathrm{m}$ ). Carrier gas, He; injector temp. $300^{\circ} \mathrm{C}$, split ratio 1:40; temp. program: $50^{\circ} \mathrm{C}$ (isothermal $1 \mathrm{~min}$ ) to $300^{\circ} \mathrm{C}$, at $20^{\circ} \mathrm{C} / \mathrm{min}$ and held isothermal for $6.5 \mathrm{~min}$ at $300^{\circ} \mathrm{C}$; FID: $300^{\circ} \mathrm{C}, \mathrm{H}_{2}: 30 \mathrm{~mL} / \mathrm{min}, \mathrm{N}_{2}: 25 \mathrm{~mL} / \mathrm{min}$, GCT-Premier: ion source: $\mathrm{EI} 70 \mathrm{eV}, 250{ }^{\circ} \mathrm{C}$; detector-voltage: $2500 \mathrm{~V}$, EI mass spectra were acquired over the mass range of $20-800 \mathrm{amu}$. 
Chiral GC analyses of the thermic cyclisation product $\mathbf{3 1}$ was carried out using an Agilent HP6890 Series chromatograph with FID as detector and hot injection. The sample was analysed on an heptakis(6- $O$-TBDMS-2,3-di- $O$-methyl)- $\beta$-cyclodextrine $(25 \mathrm{~m} \times 0.25 \mathrm{~mm}$ i.d, film thickness 0.25 $\mu \mathrm{m}$ ). Carrier gas: $\mathrm{H}_{2}$, injection: split 1:5, temp. program: $40^{\circ} \mathrm{C}$ to $180^{\circ} \mathrm{C}$ at $2^{\circ} \mathrm{C} / \mathrm{min}$ (run time $90 \mathrm{~min}$ ), FID: temp. $250{ }^{\circ} \mathrm{C}, \mathrm{H}_{2} 30 \mathrm{~mL} / \mathrm{min}$, air $350 \mathrm{~mL} / \mathrm{min}, \mathrm{N}_{2}$ make-up $20 \mathrm{~mL} / \mathrm{min}$.

Table S1: Retention time on chiral GC.

\begin{tabular}{cc}
\hline Product & $\begin{array}{c}\text { 2,3-Me-6-TBDMS- } \boldsymbol{\beta}-\mathrm{CD} \\
\text { Retention times [min] }\end{array}$ \\
\hline $\mathbf{3 1}$ & 39,42 \\
\hline
\end{tabular}

Preparative GC (pGC) were carried out with an Agilent 7890 GC System with G4513A autosampler combined with a 7693 cooling tray and hot injection. Samples were isolated on an ZB-WAX column (Phenomenex, $30 \mathrm{~m}$ x $0.53 \mathrm{~mm}$ i.d. x $3 \mu \mathrm{m}$ film thickness) and fractionated with a Gerstel PFC. The temperature gradient was optimised for each isolation, carrier gas: $\mathrm{H}_{2}(5 \mathrm{~mL} / \mathrm{min})$, injector temp.: $250^{\circ} \mathrm{C}$, splitless, injection volume: $1 \mu \mathrm{L}$, detector: FID, temperature: $275^{\circ} \mathrm{C}$. pGC with a nonpolar $\mathrm{ZB}-$ 1 column (Phenomenex, $30 \mathrm{~m} \times 0.53 \mathrm{~mm}$ i.d. $\times 3 \mu \mathrm{m}$ film thickness) was carried out on a HP 6890 chromatograph with HP 7683 autosampler and hot injection. Isolated compounds were isolated with a Gerstel PFC. The temperature gradient was optimised for each isolation, carrier gas: $\mathrm{H}_{2}(5 \mathrm{~mL} / \mathrm{min})$, injector temp.: $250{ }^{\circ} \mathrm{C}$, splitless, injection volume: $1 \mu \mathrm{L}$, detector: FID, temperature: $250^{\circ} \mathrm{C}, \mathrm{H}_{2}: 40$ $\mathrm{mL} / \mathrm{min}, \mathrm{N}_{2}: 45 \mathrm{~mL} / \mathrm{min}$.

GC-IR measurements were done on a Thermo Fisher Nicolet 6700 with an ZB-1 column (Phenomenx, $60 \mathrm{~m} \times 0.53 \mathrm{~mm}$ i.d. $\times 0,25 \mu \mathrm{m})$ coupled to a FT-IR. temperature programme: $60^{\circ} \mathrm{C}-4^{\circ} \mathrm{C} / \mathrm{min}-250$ ${ }^{\circ} \mathrm{C}$, carrier gas: $\mathrm{He}$, flow rate: $1,0 \mathrm{~mL} / \mathrm{min}$.

Preparative HPLC (pHPLC) was performed using an Agilent 1100 system with a Phenomenex Luna Silica column $(250 \mathrm{~mm} \times 10 \mathrm{~mm} \times 5 \mu \mathrm{m})$. For a continuous gradient, the solvents $n$-hexane and tertbutylmethyl ether were used. The gradient was otpimised for each separation: flow rate: $3 \mathrm{~mL} / \mathrm{min}$, temperature: room temperature, injection volume: $50 \mu \mathrm{L}$. The UV detection was carried out at $210 \mathrm{~nm}$.

Heating of reactions was performed in oil baths. Analytical thin-layer chromatography was performed using precoated silica gel plates (Macherey-Nagel, Düren) and the spots were visualised with UV light at $254 \mathrm{~nm}$ or alternatively by staining with ninhydrin, permanganate or 4-methoxybenzaldehyde solutions. ${ }^{[\mathrm{S} 1]}$ Commercially available reagents, chromatography type or dry solvents were used as received or purified by standard techniques according to the literature. ${ }^{[\mathrm{S} 1]}$ The preparation of 3,3dimethylallyl bromide is reported in reference [S2] and amine $\mathbf{S 2 4}$ in reference [S3]. Isoprene $\mathbf{S 2}$ and geraniol S5 are commercially available.

\subsection{Genes, strains, plasmids and growth conditions}

The genes used in this work were synthesised by GENEWIZ LLC as a construct with pUC57 and are optimised for expression in E. coli. The fundamental protein sequences, used for the gene synthesis, are listed in table S2. Cloning of sesquiterpene cyclases was carried out in E. coli TOP10 with pET28a(+) plasmids, leading to a $N$-terminal polyhistidine tag for the enzyme through expression. E. coli cultures were grown at $37{ }^{\circ} \mathrm{C}$ and $180 \mathrm{rpm}$ in Luria-Bertani medium (LB medium: $0.5 \%$ yeast extract, $1 \%$ tryptone/peptone, $0.05 \%$ sodium chloride) supplemented with $50 \mu \mathrm{g} \mathrm{mL}^{-1}$ kanamycine. For heterologous expression $2 \mathrm{TY}$ medium (1\% yeast extract, $1.6 \%$ tryptone/pepton, $0.5 \%$ sodium chloride) supplemented with $50 \mu \mathrm{g} \mathrm{mL}^{-1}$ kanamycin was inoculated by $2 \%$ pre-culture of $E$. coli BL21 (DE3) and grown at $37{ }^{\circ} \mathrm{C}$ and $180 \mathrm{rpm}$ to an optical density (OD) of 0.6. Then, the expression 
controlled by the T7lac-promotor was induced by adding $0.5 \mathrm{mM}$ IPTG (isopropyl- $\beta$ thiogalactopyranoside) and the temperature was decreased to $16^{\circ} \mathrm{C}$ for overnight cultivation.

Table S2: Enzymes and corresponding gene bank accession numbers used in this study.

\begin{tabular}{ccc}
\hline enzyme & gene bank accession number & reference \\
\hline Bot2 & Q6WP50 & [S4] \\
\hline kb & & \\
& &
\end{tabular}

Figure S1: Agarose gel for construct verification. After ligation with pET28a(+), the gene construct was verified by restriction digest. Marker bands were labelled in kb size.

\subsection{Protein purification}

Terpene cyclase Bot2 was overexpressed as described above. After harvesting, the cells were suspended in lysis buffer (100 mM Tris, $40 \mathrm{mM} \mathrm{NaCl}, 25 \mathrm{mM}$ imidazole), disrupted by sonification (sonotrode: KE76, I= 45\%, 30s/30s) and the histidine-tagged protein was purified by affinity chromatography. Therefore, Protino ${ }^{\circledR}$ Ni-NTA-Agarose (Macherey-Nagel GmbH \& Co. KG) was used for small amounts of protein, while large amounts were purified by ÄKTA pure 25L with a HisTrap FF column (GE Healthcare). Protein concentrations were determined by using UV/VIS spectroscopy.

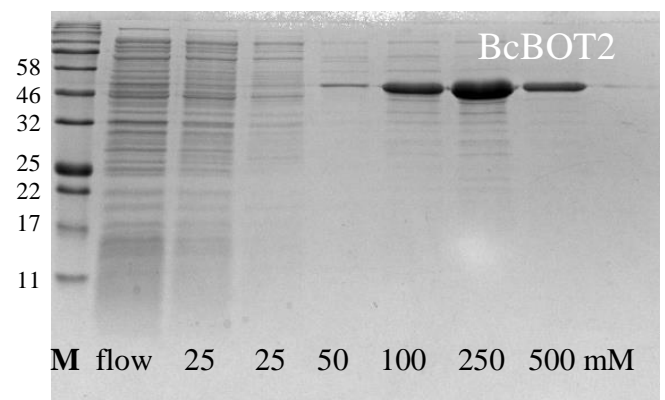

Figure S2: SDS-Page for purification of BcBOT2. Purification was done by discontinous imidazol gradient (25 mM up to $500 \mathrm{mM}$ ). As marker an Colour Prestained Protein Standard, Broad Range 10$20 \%$ Tris-glycine was used. Selected marker bands were labelled with kb size.

Enzyme purification and yield: Heterologous expression under the above mentioned conditions and protein purification by FPLC System Äkta pure (GE Healthcare, Chicago, USA) with a Ni-NTA column (HiTrap IMAC FF $5 \mathrm{~mL}, \mathrm{Ni}^{2+}$ ) gave an average enzyme yield of $220 \mathrm{mg} / \mathrm{L}$ culture for Bot2, respectively.

\subsection{Analytical enzyme tests}

Analytical enzyme tests were performed in $0.5 \mathrm{~mL}$ scale in HEPES (50 mM, pH 7.5) buffer with DTT (dithiothreitol, $5 \mathrm{mM}$ ). Purified enzyme was transferred to the chosen buffer by gel filtration with PD10 Desalting Columns (GE Healthcare) and used in a concentration of $0.01 \mathrm{~g} \mathrm{~L}^{-1}$. Then FPP (farnesyl pyrophosphate) or an unnatural substrate in concentrations of $150 \mu \mathrm{M}$ as well as $\mathrm{MgCl}_{2}(5 \mathrm{mM})$ was added. Incubation at $25^{\circ} \mathrm{C}$ (ThermoMixer) for $20 \mathrm{~min}$ was followed by extraction with $100 \mu \mathrm{L} \mathrm{n}$ hexane. Extracts were analysed by GC-MS. 


\section{Chemical syntheses}

Molecules S2, 14, S3, 15, 16, 17, S5 and S8 have been reported before including analytical data. References are given with the synthetic protocols.

2.1 Synthesis of tris(tetra- $n$-butylammonium)hydrogenpyrophosphate trihydrate (S1) ${ }^{[\mathrm{S} 5]}$ and general description of diphosphate synthesis via the chloride

a) Adjustment of the ion exchange column (DOWEX AG 50WX8, 100-200 mesh):

Ammonium form: Wash with a $\mathrm{NH}_{3}$ solution $\left(\mathrm{H}_{2} \mathrm{O}(200 \mathrm{~mL})+\right.$ conc. $\left.\mathrm{NH}_{3}(50 \mathrm{~mL})\right)$, then wash with $\mathrm{H}_{2} \mathrm{O}$ until neutral $\mathrm{pH}$.

Protonated form: Wash with $\mathrm{HCl}(3 \mathrm{M})$, then wash with $\mathrm{H}_{2} \mathrm{O}$ until neutral.

b) $1 \mathrm{~L}$ ion exchange buffer: $\mathrm{H}_{2} \mathrm{O}(980 \mathrm{~mL})+i-\mathrm{PrOH}(20 \mathrm{~mL})+\mathrm{NH}_{4} \mathrm{HCO}_{3}(2 \mathrm{~g})$

c) A solution of disodium-dihydrogenpyrophosphate $(4.44 \mathrm{~g}, 20.0 \mathrm{mmol}, 1$ eq.) and ammonia (28$30 \%, 1.42 \mathrm{~mL})$ in $\mathrm{H}_{2} \mathrm{O}(35 \mathrm{~mL})$ was eluted with water through a cartridge containing the ion exchange resin (Dowex AG 50WX8, $\mathrm{H}^{+}$-form) $(100 \mathrm{~mL})$. Then, the solution was titrated using an aqueous solution of tetra- $N$-butylammoniumhydroxide (40\%) until a $\mathrm{pH}$-value of 7.3 was reached. The solvent was removed under reduced pressure to furnish the title compound $\mathbf{S 1}(18.8 \mathrm{~g}, 19.9 \mathrm{mmol}, 99 \%)$ as a colourless solid material.

${ }^{1} \mathrm{H}-\mathrm{NMR}\left(400 \mathrm{MHz}, \mathrm{D}_{2} \mathrm{O}\right) \delta$ [ppm]: 3.13-3.06 (m, $\left.24 \mathrm{H}, \mathrm{Bu}\right), 1.57-1.47$ (m, 24 H, Bu), 1.28 (sex, $J=7.4 \mathrm{~Hz}, 24 \mathrm{H}, \mathrm{Bu}), 0.88(\mathrm{t}, J=7.4 \mathrm{~Hz}, 36 \mathrm{H}, \mathrm{Bu}) ;{ }^{13} \mathrm{C}-\mathrm{NMR}\left(100 \mathrm{MHz}, \mathrm{D}_{2} \mathrm{O}\right) \delta[\mathrm{ppm}]: 58.1(\mathrm{t}$, $J=2.8 \mathrm{~Hz}, \mathrm{Bu}), 23.4(\mathrm{Bu}), 19.3(\mathrm{t}, J=1.5 \mathrm{~Hz}, \mathrm{Bu}), 12.6(\mathrm{Bu}) ;{ }^{31} \mathrm{P}-\mathrm{NMR}\left(202 \mathrm{MHz}, \mathrm{D}_{2} \mathrm{O}\right): \delta$ [ppm]: -5.99 (s, 2 P).

The analytic data are in accordance with those published in the literature. ${ }^{\text {[S5] }}$

d) $\mathrm{N}$-Chlorosuccinimide $(1.5 \mathrm{mmol}, 1.1 \mathrm{eq}$. $)$ in dry dichloromethane was cooled to $-30{ }^{\circ} \mathrm{C}$. Then, dimethylsulfide ( $1.56 \mathrm{mmol}, 1.2$ eq.) was slowly added and stirring was continued for 5 minutes at 0 ${ }^{\circ} \mathrm{C}$. Then, the solution was cooled to $-40{ }^{\circ} \mathrm{C}$ and a solution of the allyl alcohol $(1.30 \mathrm{mmol}, 1.0 \mathrm{eq}$.) in dry dichloromethane $(2 \mathrm{~mL})$ was slowly added. The reaction mixture was stirred for $2 \mathrm{~h}$ at $0{ }^{\circ} \mathrm{C}$ and treated with brine. The aqueous phase was extracted with $n$-pentane $(3 \mathrm{x})$ and the combined organic phases were washed with brine. The organic phase was dried $\left(\mathrm{MgSO}_{4}\right)$, filtered and concentrated under reduced pressure. The resulting crude chloride was directly used in the next step. To tris(tetra- $\mathrm{N}$ butylammonium) hydrogenpyrophosphate trihydrate $\mathbf{S 1}(1.70 \mathrm{mmol}, 2.5$ eq.) in acetonitrile $(5.0 \mathrm{~mL})$ was slowly added a solution of the chloride $(0.86 \mathrm{mmol}, 1.0$ eq.) in acetonitrile $(3.5 \mathrm{~mL})$ and the reaction mixture was stirred for $2 \mathrm{~h}$ at room temperature. Then, the solvent was removed under reduced pressure and the residue was taken up in ion exchange buffer (ammonium bicarbonate $(2.0 \mathrm{~g})$ in isopropanol / water $(2 \%(\mathrm{v} / \mathrm{v}) ; 1 \mathrm{~L} ; \hat{=} 25 \mathrm{mM}$ ammonium bicarbonate) and treated with an ion exchange resin (DOWEX AG 50W-X8, 100-200 mesh, ammonium form) to provide the corresponding ammonium salt. The solvent was removed under reduced pressure.

For larger scales (> $1 \mathrm{mmol}$ of the alcohol) the eluate collected from the ion exchange purification step was first diluted with acetonitrile and then the solvent mixture was removed under reduced pressure. The residue was dissolved in a solution of ammonium bicarbonate $(0.005 \mathrm{M})$ and transferred into centrifuge tubes. A mixture of acetonitrile and isopropanol (1:1) was added. After mixing, a solid material formed which was removed by centrifugation and decanatation of the supernatant. This procedure was repeated several times and a sample of the solid residue was analysed by NMRspectroscopy in order to check the progress of ion exchange. The solid residue was purified by chromatography (?) in a buffer system consisting of ammonium bicarbonate (4.0 g) in water/ isopropanol / acetonitrile ( $3 \times 250 \mathrm{~mL} ; \hat{=} 50 \mathrm{mM}$ ammonium bicarbonate) over cellulose as stationary phase. The product was obtained as a colourless solid after lyophilisation. 


\subsection{Synthesis of farnesyldiphosphate derivative 9}

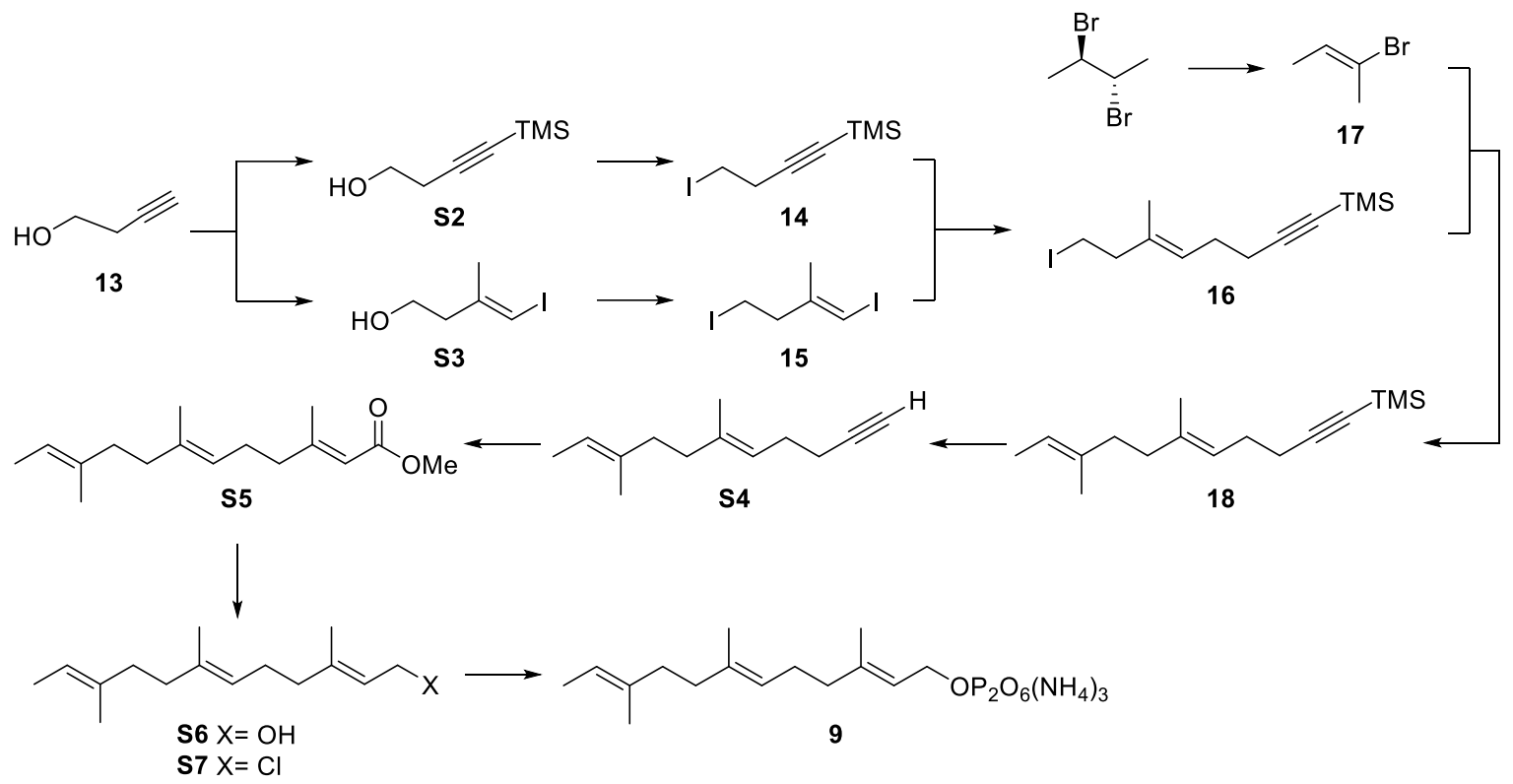

\section{4-(Trimethylsilyl)but-3-yn-1-ol (S2) ${ }^{[\mathrm{S6}]}$}

But-3-yl-1-ol (10 g, $0.14 \mathrm{~mol}, 1$ eq.) was dissolved in THF $(140 \mathrm{~mL})$ and cooled to $-78{ }^{\circ} \mathrm{C}$. n-Butyllithium (2.5 $\mathrm{M}$ in hexane, $120 \mathrm{ml}, 0.30 \mathrm{~mol}, 2.1 \mathrm{eq}$.) was added dropwise at $-78{ }^{\circ} \mathrm{C}$ and the reaction mixture was stirred for $1 \mathrm{~h}$ at room temperature. The resulting suspension was recooled to $-78{ }^{\circ} \mathrm{C}$ and chlorotrimethylsilane ( $40 \mathrm{~mL}, 0.30 \mathrm{~mol}, 2.1$ eq.) was slowly added. The reaction mixture was stirred for a another $2 \mathrm{~h}$ at room temperature. After full conversion of the starting material was observed (TLC), the reaction was terminated by the addition of water $(300 \mathrm{~mL})$ and an aqueous $\mathrm{HCl}$ solution $(1 \mathrm{M}, 150 \mathrm{~mL})$. The phases were separated and the crude product was extracted from the aqueous phase with $\mathrm{Et}_{2} \mathrm{O}$. The combined organic layers were dried over $\mathrm{MgSO}_{4}$, filtered and concentrated under reduced pressure. The crude product was purified by column chromatography (silica, petroleum ether:ethyl acetate $=10: 1)$ to yield the title compound $\mathbf{S 2}(20.30 \mathrm{~g}, 0.14 \mathrm{~mol}$, quant.) as a colourless oil.

The analytical data are in accordance with those reported in the literature. ${ }^{[\mathrm{S} 6]}$

$\mathbf{R}_{\mathbf{f}}=0.5$ (petroleum ether:ethyl acetate $\left.=4: 1\right) ;{ }^{1} \mathbf{H}-\mathbf{N M R}\left(400 \mathrm{MHz}, \mathrm{CDCl}_{3}\right): \delta=3.71(\mathrm{t}, J=6.3 \mathrm{~Hz}$, $2 \mathrm{H}, 1-\mathrm{H}), 2.50(\mathrm{t}, J=6.3 \mathrm{~Hz}, 2 \mathrm{H}, 2-\mathrm{H}), 1.75(\mathrm{bs}, 1 \mathrm{H}, \mathrm{OH}), 0.16(\mathrm{~s}, 9 \mathrm{H}, \mathrm{TMS}) \mathrm{ppm} ;{ }^{13} \mathrm{C}-\mathrm{NMR}$ (100 MHz, $\left.\mathrm{CDCl}_{3}\right): \delta=103.4$ (C-3), 87.2 (C-4), 61.0 (C-1), 24.4 (C-2), 0.21 (TMS) ppm.

\section{(4-Iodobut-1-yn-1-yl)trimethylsilane (14) ${ }^{[\mathrm{S} 7]}$}

Alcohol S2 (3 g, $21.08 \mathrm{mmol}$, 1 eq.), triphenylphosphine $(8.29 \mathrm{~g}, 31.62 \mathrm{mmol}, 1.5$ eq.) and imidazole (2.15 g, $31.62 \mathrm{mmol}, 1.5$ eq.) were dissolved in $\mathrm{CH}_{2} \mathrm{Cl}_{2}(80 \mathrm{~mL})$. The mixture was cooled to $0{ }^{\circ} \mathrm{C}$ and iodine $(8.03 \mathrm{~g}, 31.62 \mathrm{mmol}, 1.5$ eq. $)$ was added. The dark reaction mixture was stirred for $2 \mathrm{~h}$ at room temperature under the exclusion of light. After no further conversion of the starting material was observed (TLC), removal of $\mathrm{CH}_{2} \mathrm{Cl}_{2}$ under reduced pressure and addition of $n$-pentane lead to the precipitation of triphenylphosphine and triphenylphosphine oxide. The solid impurities were filtered off and the filter cake washed with $n$-pentane and $\mathrm{Et}_{2} \mathrm{O}$. If further impurities precipitated on concentration of the filtrate, the previous step was repeated until all phosphine and phosphine oxide impurities were removed. The combined organic filtrates were washed successively with a saturated aqueous $\mathrm{Na}_{2} \mathrm{SO}_{2} \mathrm{O}_{3}$ and $\mathrm{NaCl}$ solution and the phases were separated. The combined organic layers were dried over $\mathrm{MgSO}_{4}$, filtered and concentrated under reduced pressure. The crude 
product was purified by column chromatography (silica, petroleum ether) to yield the title compound 14 (4.95 g, $19.64 \mathrm{mmol}, 93 \%)$ as a colourless oil.

The analytical data are in accordance with those reported in the literature. ${ }^{[\mathrm{S} 8]}$

$\mathbf{R}_{\mathbf{f}}=0.35$ (petroleum ether); ${ }^{1} \mathbf{H}-\mathbf{N M R}\left(400 \mathrm{MHz}, \mathrm{CDCl}_{3}\right): \delta=3.22(\mathrm{t}, J=7.5 \mathrm{~Hz}, 2 \mathrm{H}, 1-\mathrm{H}), 2.79(\mathrm{t}$, $J=7.5 \mathrm{~Hz}, 2 \mathrm{H}, 2-\mathrm{H}), 0.16$ (s, $9 \mathrm{H}, \mathrm{TMS}) \mathrm{ppm} ;{ }^{13} \mathbf{C}-\mathrm{NMR}\left(100 \mathrm{MHz}, \mathrm{CDCl}_{3}\right): \delta=105.2(\mathrm{C}-4), 86.9$ (C-3), 25.2 (C-1), 1.2 (C-2), 0.1 (TMS) ppm.

\section{(E)-4-Iodo-3-methylbut-3-en-1-ol (S3) ${ }^{[S 9]}$}

To zirconocene dichloride $\left(83.4 \mathrm{mg}, 0.29 \mathrm{mmol}, 0.2\right.$ eq.) in $\mathrm{CH}_{2} \mathrm{Cl}_{2}$ (2 mL) was slowly added trimethylaluminum ( $2 \mathrm{M}$ in hexane, $2.21 \mathrm{~mL}, 4.42 \mathrm{mmol}, 3.1$ eq.) at $-25^{\circ} \mathrm{C}$ and the reaction mixture stirred for $10 \mathrm{~min}$. Subsequently, $\mathrm{H}_{2} \mathrm{O}(0.04 \mathrm{~mL}, 2.21 \mathrm{mmol}, 1.55$ eq. $)$ was added very carefully and the reaction mixture stirred for another $10 \mathrm{~min}$ at $-25^{\circ} \mathrm{C}$. In a separate flask, but-3-yl-1-ol $(100 \mathrm{mg}$, $1.43 \mathrm{mmol}, 1$ eq.) in $\mathrm{CH}_{2} \mathrm{Cl}_{2}(2 \mathrm{~mL})$ was pretreated with trimethylaluminum $(0.25 \mathrm{~mL}, 0.49 \mathrm{mmol}$, 0.34 eq.) at $0{ }^{\circ} \mathrm{C}$. The mixture was stirred for $20 \mathrm{~min}$ at $0{ }^{\circ} \mathrm{C}$ and then added dropwise to the reaction mixture. The reaction was continued for $18 \mathrm{~h}$ at room temperature, after which it was recooled to $-25^{\circ} \mathrm{C}$ and iodine (543 mg, $2.14 \mathrm{mmol}, 1.5$ eq.) in $\mathrm{CH}_{2} \mathrm{Cl}_{2}(4 \mathrm{~mL})$ was added slowly. After $2 \mathrm{~h}$, no further conversion of the starting material was observed (TLC) and the reaction was terminated by addition of a saturated aqueous $\mathrm{Na}, \mathrm{K}$-tartrate solution. The phases were separated, the aqueous phase was extracted with $\mathrm{Et}_{2} \mathrm{O}$ and the combined organic layers were washed successively with a saturated aqueous $\mathrm{Na}_{2} \mathrm{SO}_{2} \mathrm{O}_{3}$ and $\mathrm{NaCl}$ solution, dried over $\mathrm{MgSO}_{4}$, filtered and concentrated under reduced pressure. The crude product was purified by column chromatography (silica, petroleum ether:ethyl acetate $=5: 1)$ to yield the title compound $\mathbf{S 3}(247 \mathrm{mg}, 1.16 \mathrm{mmol}, 81 \%)$ as a yellow oil.

The analytical data are in accordance with those reported in the literature. ${ }^{[\mathrm{S} 10]}$

$\mathbf{R}_{\mathbf{f}}=0.35$ (petroleum ether:ethyl acetate $\left.=4: 1\right) ;{ }^{1} \mathbf{H}-\mathbf{N M R}\left(400 \mathrm{MHz}, \mathrm{CDCl}_{3}\right): \delta=6.03(\mathrm{~s}, 1 \mathrm{H}, 4-\mathrm{H})$, $3.73(\mathrm{t}, J=6.2 \mathrm{~Hz}, 2 \mathrm{H}, 1-\mathrm{H}), 2.49(\mathrm{t}, J=6.2 \mathrm{~Hz}, 2 \mathrm{H}, 2-\mathrm{H}), 1.88\left(\mathrm{~s}, 3 \mathrm{H}, \mathrm{CH}_{3}\right) \mathrm{ppm} ;{ }^{13} \mathbf{C}-\mathbf{N M R}$ $\left(100 \mathrm{MHz}, \mathrm{CDCl}_{3}\right): \delta=144.7(\mathrm{C}-3), 77.0(\mathrm{C}-4), 60.3(\mathrm{C}-1), 42.6(\mathrm{C}-2), 23.9\left(\mathrm{CH}_{3}\right) \mathrm{ppm}$.

\section{(E)-1,4-Diiodo-2-methylbut-1-ene (15) ${ }^{[\text {S11] }}$}

Vinyl iodide $\mathbf{S 3}$ ( $1 \mathrm{~g}, 4.72 \mathrm{mmol}, 1 \mathrm{eq}$ ), triphenylphosphine $(1.86 \mathrm{~g}, 7.07 \mathrm{mmol}, 1.5 \mathrm{eq}$.$) and$ imidazole (481 mg, $7.07 \mathrm{mmol}, 1.5$ eq.) were dissolved in $\mathrm{CH}_{2} \mathrm{Cl}_{2}(20 \mathrm{~mL})$. The mixture was cooled to $0{ }^{\circ} \mathrm{C}$ and iodine $(1.79 \mathrm{~g} ; 7.07 \mathrm{mmol} ; 1.5$ eq.) was added. The dark reaction mixture was stirred for $2 \mathrm{~h}$ at room temperature under the exclusion of light. After no further conversion of the starting material was observed (TLC), removal of $\mathrm{CH}_{2} \mathrm{Cl}_{2}$ under reduced pressure and addition of $n$-pentane lead to the precipitation of triphenylphosphine and triphenylphosphine oxide. The solid impurities were filtered off and the filter cake was washed with $n$-pentane and $\mathrm{Et}_{2} \mathrm{O}$. If further impurities precipitated on concentration of the filtrate, the previous step was repeated until all phosphine and phosphine oxide impurities were removed. The combined organic filtrates were washed successively with a saturated aqueous $\mathrm{Na}_{2} \mathrm{SO}_{2} \mathrm{O}_{3}$ and $\mathrm{NaCl}$ solution and the phases were separated. The combined organic layers were dried over $\mathrm{MgSO}_{4}$, filtered and concentrated under reduced pressure. The crude product was purified by column chromatography (silica, petroleum ether) to yield the title compound 15 (1.39 g, $4.32 \mathrm{mmol}, 91 \%)$ as a light pink oil.

The analytical data are consistent with those reported in the literature. ${ }^{[\mathrm{S} 8]}$

$\mathbf{R}_{\mathrm{f}}=0.6$ (petroleum ether); ${ }^{1} \mathbf{H}-\mathbf{N M R}\left(400 \mathrm{MHz}, \mathrm{CDCl}_{3}\right): \delta=6.07(\mathrm{~s}, 1 \mathrm{H}, 4-\mathrm{H}), 3.22(\mathrm{t}, J=7.5 \mathrm{~Hz}$, $2 \mathrm{H}, 1-\mathrm{H}), 2.76(\mathrm{t}, J=7.5 \mathrm{~Hz}, 2 \mathrm{H}, 2-\mathrm{H}), 1.85\left(\mathrm{~s}, 3 \mathrm{H}, \mathrm{CH}_{3}\right) \mathrm{ppm} ;{ }^{13} \mathrm{C}-\mathrm{NMR}\left(100 \mathrm{MHz}, \mathrm{CDCl}_{3}\right)$ : $\delta=146.1(\mathrm{C}-3), 77.9(\mathrm{C}-4), 43.4(\mathrm{C}-2), 23.3\left(\mathrm{CH}_{3}\right), 2.3(\mathrm{C}-1) \mathrm{ppm}$. 


\section{(E)-(8-Iodo-6-methyloct-5-en-1-yn-1-yl)trimethylsilane $(\mathbf{1 6})^{[\mathbf{S 1 1}]}$}

$t$-Butyllithium (1.9 M in hexane, $0.76 \mathrm{~mL}, 1.44 \mathrm{mmol}, 3.1$ eq.) was added dropwise to iodide $\mathbf{1 4}$ (176 mg, $0.70 \mathrm{mmol}, 1.5$ eq.) in $\mathrm{Et}_{2} \mathrm{O}(0.7 \mathrm{~mL})$ at $-78{ }^{\circ} \mathrm{C}$ and the reaction mixture stirred for $30 \mathrm{~min}$. Then, freshly flame-dried zinc bromide $(157 \mathrm{mg}, 0.70 \mathrm{mmol}, 1.5$ eq.) in THF $(0.7 \mathrm{~mL})$ was added slowly to the reaction mixture, after which it was stirred for a further $30 \mathrm{~min}$ at $0{ }^{\circ} \mathrm{C}$. In a separate flask, $\mathrm{Pd}(\mathrm{dppf}) \mathrm{Cl}_{2} \cdot\left(\mathrm{CH}_{2} \mathrm{Cl}_{2}\right)(7.6 \mathrm{mg} ; 0.01 \mathrm{mmol} ; 0.02$ eq. $)$ in THF $(0.5 \mathrm{~mL})$ was pretreated with vinyl iodide 15 (150 mg; $0.47 \mathrm{mmol}, 1$ eq.). The resulting red suspension was stirred for $20 \mathrm{~min}$ and then added to the reaction mixture. The reaction was warmed to room temperature and stirred for $3 \mathrm{~h}$. After no further conversion of the iodides were observed (TLC), the reaction was terminated by addition of $\mathrm{H}_{2} \mathrm{O}$. The phases were separated, the aqueous phase was extracted with $\mathrm{Et}_{2} \mathrm{O}$ and the combined organic layers were washed with a saturated aqueous $\mathrm{NaCl}$ solution, dried over $\mathrm{MgSO}_{4}$, filtered and concentrated under reduced pressure. The crude product was purified by column chromatography (silica, petroleum ether) to yield the title compound $16(120 \mathrm{mg}, 0.37 \mathrm{mmol}, 81 \%)$ as a light yellow oil.

The analytical data are consistent with those reported in the literature. ${ }^{\left[{ }^{[11]}\right.}$

$\mathbf{R}_{\mathbf{f}}=0.3$ (petroleum ether); ${ }^{1} \mathbf{H}-\mathbf{N M R}\left(400 \mathrm{MHz}, \mathrm{CDCl}_{3}\right): \delta=5.25(\mathrm{~m}, 1 \mathrm{H}, 4-\mathrm{H}), 3.22(\mathrm{t}, J=7.6 \mathrm{~Hz}$, $2 \mathrm{H}, 1-\mathrm{H}), 2.54(\mathrm{t}, J=7.6 \mathrm{~Hz}, 2 \mathrm{H}, 2-\mathrm{H}), 2.25(\mathrm{~m}, 4 \mathrm{H}, 5-\mathrm{H}, 6-\mathrm{H}), 1.63\left(\mathrm{~s}, 3 \mathrm{H}, \mathrm{CH}_{3}\right), 0.14(\mathrm{~s}, 9 \mathrm{H}$, TMS) ppm; ${ }^{13}$ C-NMR (100 MHz, $\mathrm{CDCl}_{3}$ ): $\delta=135.2$ (C-3), 125.7 (C-4), 107.2 (C-7), 84.7 (C-8), 43.9 (C-2), 27.5 (C-5), 20.2(C-6), $15.6\left(\mathrm{CH}_{3}\right), 4.8$ (C-1), 0.3 (TMS) ppm.

\section{(E)-2-Bromobut-2-ene (17) ${ }^{[\mathbf{S 1 2 ]}}$}

meso-2,3-Dibromobutane (9.7 g, $44.93 \mathrm{mmol}, 1$ eq.) was dissolved in ethylene glycol $(20 \mathrm{~mL})$ and the reaction solution heated to $120{ }^{\circ} \mathrm{C}$. Potassium hydroxide $(3.02 \mathrm{~g}, 56.11 \mathrm{mmol}, 1.2$ eq.) dissolved in ethylene glycol $(50 \mathrm{~mL})$ was added dropwise and the reaction mixture was stirred under reflux for $2 \mathrm{~h}$. After distillative isolation and drying over $\mathrm{MgSO}_{4}$, title compound 17 (4.9 g, $36.27 \mathrm{mmol}, 81 \%$ ) was obtained as a colourless, volatile oil.

The analytical data are in accordance with those reported in the literature. ${ }^{[\mathrm{S} 9]}$

${ }^{1} \mathbf{H}-\mathbf{N M R}\left(400 \mathrm{MHz}, \mathrm{CDCl}_{3}\right.$ ): $\delta=5.88$ (qq, $J=7.1 \mathrm{~Hz}, 1.4 \mathrm{~Hz}, 1 \mathrm{H}, 3-\mathrm{H}$ ), 2.20 (qi, $J=1.2 \mathrm{~Hz}, 3 \mathrm{H}$, $1-\mathrm{H}), 1.61(\mathrm{dq}, J=6.9 \mathrm{~Hz}, 1.2 \mathrm{~Hz}, 3 \mathrm{H}, 4-\mathrm{H}) \mathrm{ppm} ;{ }^{13} \mathbf{C}-\mathrm{NMR}\left(100 \mathrm{MHz}, \mathrm{CDCl}_{3}\right): \delta=126.7(\mathrm{C}-3)$, 119.6 (C-2), 22.9 (C-1), 15.1 (C-4) ppm.

\section{((5E,9E)-6,9-Dimethylundeca-5,9-dien-1-yn-1-yl)trimethylsilane (18)}

$t$-Butyllithium (1.9 M in hexane, $3.62 \mathrm{~mL}, 6.87 \mathrm{mmol}, 2.2$ eq.) was added dropwise to iodide $\mathbf{1 6}$ $\left(1.0 \mathrm{~g}, 3.12 \mathrm{mmol}, 1\right.$ eq.) in $\mathrm{Et}_{2} \mathrm{O}(3 \mathrm{~mL})$ at $-78^{\circ} \mathrm{C}$ and the reaction mixture stirred for $30 \mathrm{~min}$. Then, freshly flame-dried zinc bromide ( $1.05 \mathrm{~g}, 4.68 \mathrm{mmol}, 1.5 \mathrm{eq}$.) in THF $(5 \mathrm{~mL})$ was added slowly to the reaction mixture, after which it was stirred for a further $30 \mathrm{~min}$ at $0{ }^{\circ} \mathrm{C}$. In a separate flask, $\mathrm{Pd}(\mathrm{dppf}) \mathrm{Cl}_{2} \cdot\left(\mathrm{CH}_{2} \mathrm{Cl}_{2}\right)(127 \mathrm{mg} ; 0.16 \mathrm{mmol} ; 0.05 \mathrm{eq}$. $)$ in THF $(6 \mathrm{~mL})$ was pretreated with vinyl bromide 17 ( $843 \mathrm{mg} ; 6.24 \mathrm{mmol}, 2$ eq.). The resulting red suspension was stirred for $20 \mathrm{~min}$ and then added to the reaction mixture. The reaction was warmed to room temperature and stirred for $3 \mathrm{~h}$. After no further conversion of the substrates were observed (TLC), the reaction was terminated by addition of $\mathrm{H}_{2} \mathrm{O}$. The phases were separated, the aqueous phase was extracted with $\mathrm{Et}_{2} \mathrm{O}$ and the combined organic layers were washed with a saturated aqueous $\mathrm{NaCl}$ solution, dried over $\mathrm{MgSO}_{4}$, filtered and concentrated under reduced pressure. The crude product was purified by column chromatography (silica, petroleum ether) to yield the title compound $18(574 \mathrm{mg}, 2.31 \mathrm{mmol}, 74 \%)$ as a colourless oil.

$\mathbf{R}_{\mathbf{f}}=0.45$ (petroleum ether); ${ }^{1} \mathbf{H}-\mathbf{N M R}\left(400 \mathrm{MHz}, \mathrm{CDCl}_{3}\right): \delta=5.25-5.12(\mathrm{~m}, 2 \mathrm{H}, 1-\mathrm{H}, 6-\mathrm{H}), 2.24-2.22$ (m, $\left.4 \mathrm{H}, 2 \times \mathrm{CH}_{2}\right), 2.08\left(\mathrm{~m}, 4 \mathrm{H}, 2 \times \mathrm{CH}_{2}\right), 1.64\left(\mathrm{~m}, 3 \mathrm{H}, \mathrm{CH}_{3}\right), 1.62\left(\mathrm{~m}, 3 \mathrm{H}, \mathrm{CH}_{3}\right), 1.59(\mathrm{~d}, J=7 \mathrm{~Hz}$, $3 \mathrm{H}, 1-\mathrm{Me}$ ), 0.17 (s, $9 \mathrm{H}, \mathrm{TMS}) \mathrm{ppm} ;{ }^{13} \mathrm{C}-\mathrm{NMR}\left(100 \mathrm{MHz}, \mathrm{CDCl}_{3}\right): \delta=136.9$ (C-5), 135.9 (C-2), 122.6 (C-6), 118.4 (C-1), 107.6 (C-9), 84.4 (C-10), 38.5 (C-3, C-4), 27.5 (C-7), 20.5 (C-8), 16.3 (Me), 
$15.8(\mathrm{Me}), 13.5$ (Me), 0.3 (TMS) ppm; HRMS [EI] $\mathrm{m} / z$ calculated for $\mathrm{C}_{15} \mathrm{H}_{25} \mathrm{Si}\left[\mathrm{M}-\mathrm{CH}_{3}\right]$ 233.1726, found 233.1726 .

\section{(5E,9E)-6,9-Dimethylundeca-5,9-dien-1-yne (S4)}

To compound 18 (50 $\mathrm{mg}, \quad 0.20 \mathrm{mmol}, 1 \mathrm{eq}$.) dissolved in an aqueous methanol solution ( $\mathrm{MeOH}: \mathrm{H}_{2} \mathrm{O}=9: 1,1 \mathrm{~mL}$ ) was added potassium hydroxide (34 mg, $0.6 \mathrm{mmol}, 3 \mathrm{eq}$.) and the reaction mixture stirred for $4 \mathrm{~h}$ at room temperature. After no further conversion of the starting material was observed (TLC), $\mathrm{Et}_{2} \mathrm{O}$ was added. The phases were separated, the aqueous phase was extracted with $\mathrm{Et}_{2} \mathrm{O}$ and the combined organic layers were washed with a saturated aqueous $\mathrm{NaCl}$ solution, dried over $\mathrm{MgSO}_{4}$, filtered and concentrated under reduced pressure. The crude colourless oil was directly used in the next step without further purification.

\section{Methyl (2E,6E,10E)-3,7,10-trimethyldodeca-2,6,10-trienoate (S5) ${ }^{[\mathrm{S} 13]}$}

Trimethylaluminum ( $2 \mathrm{M}$ in hexane, $0.2 \mathrm{~mL}, 0.4 \mathrm{mmol}, 2$ eq.) was slowly added to zirconocene dichloride (58 mg, $0.2 \mathrm{mmol}, 1$ eq.) in $\mathrm{CH}_{2} \mathrm{Cl}_{2}(1 \mathrm{~mL})$ at $0{ }^{\circ} \mathrm{C}$. After stirring for $10 \mathrm{~min}$, alkyne $\mathbf{S} 4$ ( $35 \mathrm{mg}, 0.2 \mathrm{mmol}, 1$ eq.) in $\mathrm{CH}_{2} \mathrm{Cl}_{2}(1 \mathrm{~mL})$ was added and the mixture stirred for $20 \mathrm{~h}$ at room temperature. Then, methyl chloroformate $(0,08 \mathrm{~mL}, 0,99 \mathrm{mmol}, 5 \mathrm{eq}$.) was slowly added and the reaction was stirred for another $16 \mathrm{~h}$ at room temperature. After no further conversion of the starting material was observed (TLC), the reaction was terminated by the addition of a saturated aqueous $\mathrm{NH}_{4} \mathrm{Cl}$ solution. The phases were separated, the aqueous phase was extracted with $\mathrm{Et}_{2} \mathrm{O}$ and the combined organic layers were washed with a saturated aqueous $\mathrm{NaCl}$ solution, dried over $\mathrm{MgSO}_{4}$, filtered and concentrated under reduced pressure. The crude product was purified by column chromatography (silica, petroleum ether:ethyl acetate $=50: 1 \rightarrow 20: 1$ ) to yield the title compound $\mathbf{S 5}$ (38 $\mathrm{mg}, 0.15 \mathrm{mmol}, 77 \%$ over two steps) as a colourless oil.

$\mathbf{R}_{\mathbf{f}}=0.45$ (petroleum ether:ethyl acetate $\left.=50: 1\right) ;{ }^{1} \mathbf{H}-\mathbf{N M R}\left(400 \mathrm{MHz}, \mathrm{CDCl}_{3}\right): \delta=5.66(\mathrm{~s}, 1 \mathrm{H}, 10-\mathrm{H})$, 5.66 (q, $J=6.5 \mathrm{~Hz}, 1 \mathrm{H}, 1-\mathrm{H}), 5.07$ (m, $1 \mathrm{H}, 6-\mathrm{H}), 3.68$ (s, $3 \mathrm{H}, \mathrm{OMe}), 2.16-2.15$ (m, 7 H, 7-H, 8-H, 9Me), 2.04 (m, 4 H, 3-H, 4-H), 1.59-1.58 (m, 6 H, 2-Me, 5-Me), 1.56 (m, 3 H, 1-Me) ppm; ${ }^{13} \mathbf{C}-\mathbf{N M R}$ (100 MHz, $\left.\mathrm{CDCl}_{3}\right): \delta=167.4(\mathrm{C}-\mathrm{OOMe}), 160.3$ (C-9), 136.5 (C-5), 135.9 (C-2), 122.9 (C-6), 118.4 (C-1), 115.3 (C-10), 50.9 (OMe), 41.1 (C-8), 38.5 (C-3, C-4), 38.5 (C-3, C-4), 26.1 (C-7), 19.0 (9-Me), 16.1 (5-Me), 15.8 (2-Me), 13.5 (1-Me), ppm; HRMS [ESI] $\mathrm{m} / z$ calculated for $\mathrm{C}_{16} \mathrm{H}_{26} \mathrm{O}_{2}+\mathrm{Na}^{+}\left[\mathrm{M}+\mathrm{Na}^{+}\right]$273.1831, found 273.1837.

\section{(2E,6E,10E)-3,7,10-Trimethyldodeca-2,6,10-triene-1-ol (S6)}

Methyl ester $\mathbf{S 5}\left(100 \mathrm{mg}, 0.40 \mathrm{mmol}, 1\right.$ eq.) in $\mathrm{CH}_{2} \mathrm{Cl}_{2}(2 \mathrm{~mL})$ was cooled to $0{ }^{\circ} \mathrm{C}$ and slowly treated with diisobutylaluminium hydride $(0.88 \mathrm{~mL}, 0.88 \mathrm{mmol}, 2.2$ eq. $)$. After stirring the reaction mixture at room temperature for $2 \mathrm{~h}$, no further conversion of the starting material was observed (TLC). The reaction was terminated by the addition of a saturated aqueous $\mathrm{K}-$, Na-tartrate solution. After stirring for $18 \mathrm{~h}$, the phases were separated and the aqueous phase was extracted with $\mathrm{Et}_{2} \mathrm{O}$. The combined organic layers were washed with a saturated aqueous $\mathrm{NaCl}$ solution, dried over $\mathrm{MgSO}_{4}$, filtered and concentrated under reduced pressure. The crude product was purified by column chromatography ( silica, petroleum ether:ethyl acetate $=10: 1 \rightarrow 5: 1)$ to yield the title compound $\mathbf{S 6}$ (70 mg, $0.31 \mathrm{mmol}, 80 \%)$ as a colourless oil.

$\mathbf{R}_{\mathbf{f}}=0.55$ (petroleum ether:ethyl acetate $\left.=3: 1\right) ;{ }^{1} \mathbf{H}-\mathbf{N M R}\left(400 \mathrm{MHz}, \mathrm{CDCl}_{3}\right): \delta=5.41(\mathrm{tq}, J=6.9 \mathrm{~Hz}$, $1.3 \mathrm{~Hz}, 1 \mathrm{H}, 10-\mathrm{H}), 5.19$ (q, $J=6.6 \mathrm{~Hz}, 1 \mathrm{H}, 1-\mathrm{H}), 5.10(\mathrm{t}, J=6.7 \mathrm{~Hz}, 1 \mathrm{H}, 6-\mathrm{H}), 4.15$ (d, $J=6.9 \mathrm{~Hz}$, $2 \mathrm{H}, 11-\mathrm{H}), 2.13-2.01$ (m, 8 H, 3-H, 4-H, 7-H, 8-H), 1.68 (m, 3 H, 9-Me), 1.59 (m, 6 H, 2-Me, 5-Me), $1.56(\mathrm{~d}, J=6.6 \mathrm{~Hz}, 3 \mathrm{H}, 1-\mathrm{Me}) \mathrm{ppm} ;{ }^{13} \mathrm{C}-\mathrm{NMR}\left(100 \mathrm{MHz}, \mathrm{CDCl}_{3}\right): \delta=140.0(\mathrm{C}-9), 136.0(\mathrm{C}-5)$, 135.7 (C-2), 123.8 (C-10), 123.5 (C-6), 118.4 (C-1), 115.3 (C-10), 59.6 (C-11), 39.7 (C-8), 38.6 (C-3, C-4), 38.5 (C-3, C-4), 26.4 (C-7), 16.4 (9-Me), 16.1 (5-Me), 15.8 (2-Me), 13.5 (1-Me), ppm; HRMS [ESI] $m / z$ calculated for $\mathrm{C}_{15} \mathrm{H}_{26} \mathrm{O}+\mathrm{Na}^{+}[\mathrm{M}+\mathrm{Na}]^{+} 245.1881$, found 245.1882 . 
(2E,6E,10E)-1-chloro-3,7,10-trimethyldodeca-2,6,10-triene (S7)

Allyl chloride $\mathbf{S 7}$ was prepared according to the general procedure for the synthesis of allyl chlorides starting from allyl alcohol $\mathbf{S 6}$ (60 mg, $0.27 \mathrm{mmol}, 1 \mathrm{eq}$.) and was obtained as a yellow oil.

$\mathbf{R}_{\mathbf{f}}=0.8$ (petroleum ether:ethyl acetate $=4: 1$ ); HRMS [ESI] $\mathrm{m} / z$ calculated for $\mathrm{C}_{15} \mathrm{H}_{25} \mathrm{Cl}+\mathrm{Na}^{+}$ $[\mathrm{M}+\mathrm{Na}]^{+}$263.1542, found 263.1549 .

\section{(2E,6E,10E)-3,7,10-Trimethyldodeca-2,6,10-trien-1-yl diphosphate (9)}

Starting from allyl chloride $\mathbf{S} 7$ (64 $\mathrm{mg}, 0.27 \mathrm{mmol}$, 1 eq.), the title compound $\mathbf{9}$ was prepared according to the general procedure for the synthesis of diphosphates. Diphosphate 9 (86 $\mathrm{mg}, 0.20$ mmol, $75 \%$ over two steps) was obtained as a colourless semisolid foam containing minor impurities of diphosphate salt $\mathbf{S 1}$.

${ }^{1} \mathbf{H}-N M R\left(400 \mathrm{MHz}, \mathrm{D}_{2} \mathrm{O}\right): \delta=5.38(\mathrm{t}, J=6.6 \mathrm{~Hz}, 1 \mathrm{H}, 10-\mathrm{H}), 5.21(\mathrm{q}, J=6.6 \mathrm{~Hz}, 1 \mathrm{H}, 1-\mathrm{H}), 5.14(\mathrm{t}$, $J=6.8 \mathrm{~Hz}, 1 \mathrm{H}, 6-\mathrm{H}), 4.39(\mathrm{t}, J=6.6 \mathrm{~Hz}, 2 \mathrm{H}, 11-\mathrm{H}), 2.12-2.05(\mathrm{~m}, 8 \mathrm{H}, 3-\mathrm{H}, 4-\mathrm{H}, 7-\mathrm{H}, 8-\mathrm{H}), 1.63$ (m, $3 \mathrm{H}, 9-\mathrm{Me}), 1.58-1.46$ (m, $9 \mathrm{H}, 1-\mathrm{Me}, 2-\mathrm{Me}, 5-\mathrm{Me}) \mathrm{ppm} ;{ }^{13} \mathbf{C}-\mathrm{NMR}\left(500 \mathrm{MHz}, \mathrm{D}_{2} \mathrm{O}\right): \delta=142.7$ (C-9), 137.2 (C-5), 136.8 (C-2), 124.3 (C-6), 119.9 (d, C-10), 119.0 (C-1), 62.4(d, C-10), 38.8 (C-8), 37.4 (d, C-3, C-4), 25.6 (C-7), 15.6 (5-Me), 15.1 (9-Me), 14.7 (2-Me), 12.6 (1-Me) ppm; ${ }^{31} \mathbf{P}-\mathbf{N M R}$ $\left(160 \mathrm{MHz}, \mathrm{D}_{2} \mathrm{O}\right): \delta=-6.30(\mathrm{~d}, J=22.3 \mathrm{~Hz}),-10.04(\mathrm{~d}, J=22.0 \mathrm{~Hz}) \mathrm{ppm} ;$ HRMS [ESI] $\mathrm{m} / z$ calculated for $\mathrm{C}_{15} \mathrm{H}_{27} \mathrm{O}_{7} \mathrm{P}_{2}\left[\mathrm{M}-3 \times \mathrm{NH}_{3}\right]$ 281.1232, found 281.1232.

\subsection{Synthesis of farnesylpyrophosphate derivative 10}

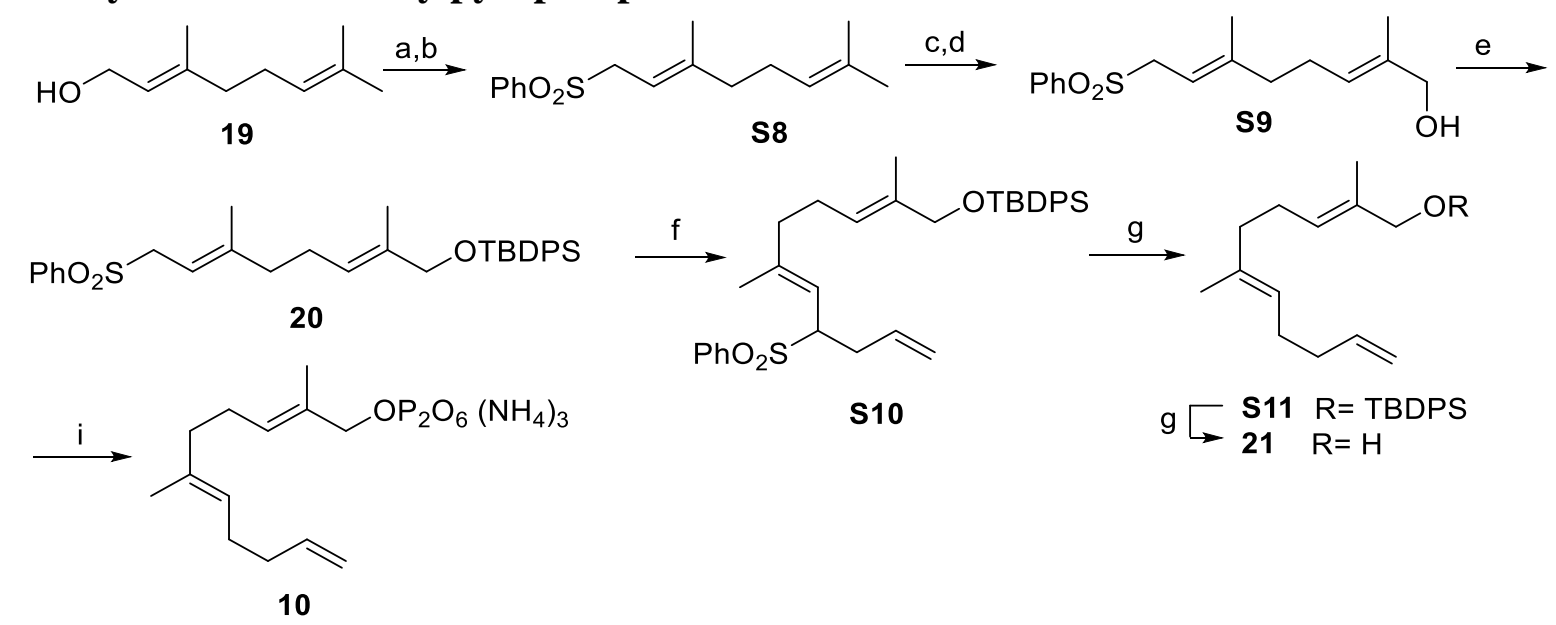

\section{(E)-[(3,7-Dimethylocta-2,6-diene-1-yl)sulfonyl]benzene (S8)}

Tetrabromomethane $\left(29.2 \mathrm{~g}, 88.0 \mathrm{mmol}, 1.10\right.$ equiv.) was added at $-20{ }^{\circ} \mathrm{C}$ to a solution of geraniol (19) (12.3 g, 80.0 mmol, 1.0 equiv.) and $\mathrm{PPh}_{3}$ (23.1 g, 88.0 mmol, 1.10 equiv.) in $\mathrm{CH}_{2} \mathrm{Cl}_{2}(30 \mathrm{~mL})$ under a nitrogen atmosphere and then stirred for $4.5 \mathrm{~h}$. Then, $\mathrm{n}$-pentane $(200 \mathrm{~mL})$ was added and the precipitate was filtered off. The solvent of the filtrate was removed under reduced pressure. Again npentane was added and the process was repeated until no more solids precipitated. The resulting oil was dripped into a suspension of sodium benzene sulphinate ( $14.5 \mathrm{~g}, 88.0 \mathrm{mmol}, 1.10$ equiv.) in DMF $(65 \mathrm{~mL})$ at ambient temperature and stirred for $20 \mathrm{~h} . \mathrm{H}_{2} \mathrm{O}(50 \mathrm{~mL})$, a total aqueous $\mathrm{NaHCO}_{3}$ solution $(80 \mathrm{~mL})$ and tert-butylmethyl ether $(70 \mathrm{~mL})$ were added, the organic phase separated and the aqueous phase extracted with tert-butylmethyl ether $(2 \times 50 \mathrm{~mL})$. The combined organic phases were washed with $\mathrm{H}_{2} \mathrm{O}$ and brine, dried over $\mathrm{MgSO}_{4}$, filtered and the solvent removed under reduced pressure. Column chromatographic purification on silica gel $(\mathrm{PE} / \mathrm{EtOAc}=15: 1)$ provided the title compound $\mathbf{S 8}$ $(13.7 \mathrm{~g}, 49.1 \mathrm{mmol}, 61 \%)$ as a colourless oil.

${ }^{1}$ H-NMR $\left(400 \mathrm{MHz}, \mathrm{CDCl}_{3}\right): \delta(\mathrm{ppm})=7.89-7.84(\mathrm{~m}, 2 \mathrm{H}, \mathrm{Ar}), 7.66-7.60(\mathrm{~m}, 1 \mathrm{H}, \mathrm{Ar}), 7.55-7.50$ (m, $2 \mathrm{H}, \mathrm{Ar}), 5.21-5.15(\mathrm{~m}, 1 \mathrm{H}, 2-\mathrm{H}), 5.06-5.00(\mathrm{~m}, 1 \mathrm{H}, 6-\mathrm{H}), 3.80(\mathrm{~d}, J=8.0 \mathrm{~Hz}, 2 \mathrm{H}, 1-\mathrm{H}), 2.01-$ 
1.98 (m, 4 H, 4-H, 5-H), 1.68 (s, 3 H, 7-Me), 1.58 (s, 3 H, 7-Me), 1.30 (s, $3 \mathrm{H}, 3-\mathrm{Me}$ ); ${ }^{13} \mathrm{C}-\mathbf{N M R}$ $\left(100 \mathrm{MHz}, \mathrm{CDCl}_{3}\right): \delta(\mathrm{ppm})=146.5(\mathrm{Ar}), 138.8(\mathrm{C}-3), 133.6(\mathrm{Ar}), 132.2(\mathrm{C}-7), 129.1$ (Ar), 128.7 (Ar), 123.6 (C-6), 110.4 (C-2), 56.2 (C-1), 39.8 (C-4), 26.3 (C-5), 25.8 (7-Me), 17.8 (7-Me), 16.3 (3-Me).

These data correspond to the data given in the literature. ${ }^{[\mathrm{S} 14]}$

\section{(2E,6E)-2,6-Dimethyl-8-(phenylsulfonyl)octa-2,6-diene-1-ol (S9)}

A suspension of selenium dioxide $(0.54 \mathrm{~g}, 4.91 \mathrm{mmol}, 0.10$ equiv. $)$ and salicylic acid $(0.68 \mathrm{~g}, 4.91$ mmol, 0.10 equiv.) in $\mathrm{CH}_{2} \mathrm{Cl}_{2}(50 \mathrm{~mL})$ was mixed with a solution of tert- $\mathrm{BuOOH}\left(70 \%\right.$ in $\mathrm{H}_{2} \mathrm{O}, 23.6$ $\mathrm{mL}, 172 \mathrm{mmol}, 3.50$ equiv.) at ambient temperature and stirred for $10 \mathrm{~min}$. Sulfone $\mathbf{S 7}$ (13.7 g, 49.1 mmol, 1.00 equiv.) was then added and the reaction mixture stirred for $20 \mathrm{~h} . \mathrm{H}_{2} \mathrm{O}(50 \mathrm{~mL})$ and a saturated aqueous $\mathrm{NaHCO}_{3}$ solution $(50 \mathrm{~mL})$ were added, the organic phase was separated and the aqueous phase extracted with tert-butylmethyl ether $(2 \times 100 \mathrm{~mL})$. The combined organic phases were dried over $\mathrm{MgSO}_{4}$, filtered and the solvent removed under reduced pressure.

The crude product was taken up in $\mathrm{MeOH}(50 \mathrm{~mL})$, mixed portionwise with sodium borohydride (0.93 g, $24.6 \mathrm{mmol}, 0.50$ equivalent) at $0{ }^{\circ} \mathrm{C}$ and then stirred for $30 \mathrm{~min} . \mathrm{H}_{2} \mathrm{O}(5 \mathrm{~mL})$ was added and the mixture concentrated under reduced pressure. $\mathrm{H}_{2} \mathrm{O}(50 \mathrm{~mL})$ and tert-butylmethyl ether $(50 \mathrm{~mL})$ were added, the organic phase was separated and the aqueous phase extracted with tert-butylmethyl ether (2 $\times 50 \mathrm{~mL}$ ). The combined organic phases were dried over $\mathrm{MgSO}_{4}$, filtered and the solvent was removed under reduced pressure. Column chromatographic purification on silica gel (PE/EtOAc $=8: 1 \rightarrow 4: 1 \rightarrow$ 2:1) provided the title compound $\mathbf{S 9}(9.23 \mathrm{~g}, 31.4 \mathrm{mmol}, 64 \%)$ as a colourless oil.

${ }^{1} \mathbf{H}-\mathrm{NMR}\left(400 \mathrm{MHz}, \mathrm{CDCl}_{3}\right): \delta(\mathrm{ppm})=7.89-7.84(\mathrm{~m}, 2 \mathrm{H}, \mathrm{Ar}), 7.66-7.61(\mathrm{~m}, 1 \mathrm{H}, \mathrm{Ar}), 7.57-7.50$ $(\mathrm{m}, 2 \mathrm{H}, \operatorname{Ar}), 5.36-5.30(\mathrm{~m}, 1 \mathrm{H}, 3-\mathrm{H}), 5.22-5.16(\mathrm{~m}, 1 \mathrm{H}, 7-\mathrm{H}), 3.99(\mathrm{~s}, 2 \mathrm{H}, 1-\mathrm{H}), 3.79(\mathrm{~d}$, $J=7.9 \mathrm{~Hz}, 2 \mathrm{H}, 8-\mathrm{H}), 2.15-2.02(\mathrm{~m}, 4 \mathrm{H}, 4-\mathrm{H}, 5-\mathrm{H}), 1.65$ (s, $3 \mathrm{H}, 2-\mathrm{Me}), 1.37$ (s, $3 \mathrm{H}, 6-\mathrm{Me}) ;{ }^{13} \mathrm{C}-$ NMR (100 MHz, CDCl $): \delta(\mathrm{ppm})=146.2(\mathrm{Ar}), 139.0(\mathrm{C}-6), 135.7(\mathrm{C}-2), 133.7(\mathrm{Ar}), 129.2(\mathrm{Ar})$, 128.5 (Ar), 124.8 (C-3), 110.5 (C-7), 68.8 (C-1), 56.2 (C-8), 39.3 (C-5), 25.6 (C-4), 16.3 (6-Me), 13.8 (2-Me).

These data correspond to the data given in the literature. ${ }^{\left[{ }^{[15]}\right.}$

\section{$t$-Butyl $\{[(2 E, 6 E)-2,6$-dimethyl-8-(phenylsulfonyl)octa-2,6-diene-1-yl]oxy\}diphenylsilane (20)}

A solution of alcohol S9 (3.00 g, $10.2 \mathrm{mmol}, 1.0$ equiv.) and imidazole $(1.74 \mathrm{~g}, 25.5 \mathrm{mmol}, 2.50$ equiv.) in $\mathrm{CH}_{2} \mathrm{Cl}_{2}(11 \mathrm{~mL})$ was mixed with TBDPSCl (2.74 mL, $10.7 \mathrm{mmol}, 1.05$ equiv.) at ambient temperature in a nitrogen atmosphere and then stirred for $2 \mathrm{~h}$. A saturated aqueous $\mathrm{NH}_{4} \mathrm{Cl}$ solution $(25$ $\mathrm{mL}$ ) was added, the organic phase separated and the aqueous phase extracted with tert-butylmethyl ether $(2 \times 20 \mathrm{~mL})$. The combined organic phases were washed with a total aqueous $\mathrm{NH}_{4} \mathrm{Cl}$ solution (30 $\mathrm{mL}$ ), dried over $\mathrm{MgSO}_{4}$, filtered and the solvent removed under reduced pressure. Column chromatographic purification on silica gel $(\mathrm{PE} /$ EtOAc $=20: 1 \rightarrow 15: 1 \rightarrow 10: 1)$ provided the title compound 20 (4.86 g, $9.11 \mathrm{mmol}, 89 \%)$ as a colourless oil.

${ }^{1} \mathbf{H}-\mathbf{N M R}\left(400 \mathrm{MHz}, \mathrm{CDCl}_{3}\right): \delta(\mathrm{ppm})=7.89-7.84(\mathrm{~m}, 2 \mathrm{H}, \mathrm{Ar}), 7.69-7.65(\mathrm{~m}, 4 \mathrm{H}, \mathrm{Ar}), 7.64-7.59$ (m, $1 \mathrm{H}, \mathrm{Ar}), 7.55-7.49$ (m, $2 \mathrm{H}, \mathrm{Ar}), 7.45-7.34$ (m, $6 \mathrm{H}, \mathrm{Ar}), 5.39-5.32(\mathrm{~m}, 1 \mathrm{H}, 3-\mathrm{H}), 5.23-5.16$ (m, $1 \mathrm{H}, 7-\mathrm{H}), 4.03$ (s, $2 \mathrm{H}, 1-\mathrm{H}), 3.79$ (d, $J=7.9 \mathrm{~Hz}, 2 \mathrm{H}, 8-\mathrm{H}), 2.11-1.98$ (m, $4 \mathrm{H}, 4-\mathrm{H}, 5-\mathrm{H}), 1.58$ (s, $3 \mathrm{H}, \mathrm{Me}), 1.32$ (s, $3 \mathrm{H}, \mathrm{Me}), 1.05$ (s, $9 \mathrm{H}, \mathrm{TBDPS}) ;{ }^{13} \mathbf{C}-\mathbf{N M R}\left(100 \mathrm{MHz}, \mathrm{CDCl}_{3}\right) \delta(\mathrm{ppm})=146.4$ (Ar), 138.8 (C-6), 135.7 (Ar), 134.7(C-2), 133.9 (Ar), 133.7 (Ar), 129.7 (Ar), 129.1 (Ar), 128.7 (Ar), 127.7 (Ar), 123.3 (C-3), 110.6 (C-7), 68.9 (C-1), 56.2 (C-8), 39.6 (C-5), 27.0 (TBDPS), 25.8 (C-4), 19.5 (TBDPS), 16.3 (Me), 13.7 (Me); HRMS [ESI] $\mathrm{m} / z$ calculated for $\mathrm{C}_{18} \mathrm{H}_{24} \mathrm{O}_{4} \mathrm{~S}+\mathrm{Na}^{+}[\mathrm{M}+\mathrm{Na}]^{+}$ 359.1293, found 359.1291. 


\section{$t$-Butyl-\{[(2E,6E)-2,6-dimethyl-8-(phenylsulfonyl)undeca-2,6,10-triene-1-yl]oxy $\}$ - diphenylsilane (S10)}

A solution of sulfone 20 (3.46 g, $6.50 \mathrm{mmol}, 1.0$ equiv.) in DME (20 mL) was mixed dropwise with KHMDS ( $0.5 \mathrm{M}$ in toluene, $13.7 \mathrm{~mL}, 6.83 \mathrm{mmol}, 1.05$ equiv.) at $-40{ }^{\circ} \mathrm{C}$ in a nitrogen atmosphere and then stirred for $0.5 \mathrm{~h}$. Allyl bromide (24a) $(0.84 \mathrm{~mL}, 9.75 \mathrm{mmol}, 1.50$ equiv.) was then added dropwise and the mixture stirred for another $3 \mathrm{~h}$. A saturated aqueous $\mathrm{NH}_{4} \mathrm{Cl}$ solution $(30 \mathrm{~mL}), \mathrm{H}_{2} \mathrm{O}(30 \mathrm{~mL})$ and tert-butylmethyl ether $(30 \mathrm{~mL})$ were added, the organic phase was separated and the aqueous phase extracted with tert-butylmethyl ether $(2 \times 30 \mathrm{~mL})$. The combined organic phases were dried over $\mathrm{MgSO}_{4}$, filtered and the solvent removed under reduced pressure. Column chromatographic purification on silica gel $(\mathrm{PE} / \mathrm{EtOAc}=25: 1 \rightarrow 20: 1)$ provided the title compound $\mathbf{S 1 0}(3.54 \mathrm{~g}, 6.18$ mmol, $95 \%)$ as a colourless oil.

${ }^{1} \mathbf{H}-\mathbf{N M R}\left(400 \mathrm{MHz}, \mathrm{CDCl}_{3}\right): \delta(\mathrm{ppm})=7.86-7.82(\mathrm{~m}, 2 \mathrm{H}, \mathrm{Ar}), 7.69-7.65(\mathrm{~m}, 4 \mathrm{H}, \mathrm{Ar}), 7.64-7.58$ (m, $1 \mathrm{H}, \mathrm{Ar}), 7.53-7.47(\mathrm{~m}, 2 \mathrm{H}, \mathrm{Ar}), 7.45-7.34(\mathrm{~m}, 6 \mathrm{H}, \mathrm{Ar}), 5.63$ (ddt, $J=17.0,10.1,7.0 \mathrm{~Hz}, 1 \mathrm{H}$, 10-H), 5.36-5.30 (m, $1 \mathrm{H}, 3-\mathrm{H}), 5.10-5.04\left(\mathrm{~m}, 1 \mathrm{H}, 11-\mathrm{H}_{\mathrm{b}}\right), 5.02-4.96\left(\mathrm{~m}, 2 \mathrm{H}, 7-\mathrm{H}, 11-\mathrm{H}_{\mathrm{a}}\right), 4.03(\mathrm{~s}$, $2 \mathrm{H}, 1-\mathrm{H}), 3.80(\mathrm{td}, J=10.5,3.5 \mathrm{~Hz}, 1 \mathrm{H}, 8-\mathrm{H}), 2.96-2.87\left(\mathrm{~m}, 1 \mathrm{H}, 9-\mathrm{H}_{\mathrm{a}}\right), 2.45-2.35\left(\mathrm{~m}, 1 \mathrm{H}, 9-\mathrm{H}_{\mathrm{b}}\right)$, 2.06-1.93 (m, 4 H, 4-H, 5-H), 1.59 (s, $3 \mathrm{H}, \mathrm{Me}), 1.20$ (d, $J=1.2 \mathrm{~Hz}, 3 \mathrm{H}, \mathrm{Me}$ ), 1.06 (s, 9 H, TBDPS); ${ }^{13}$ C-NMR $\left(100 \mathrm{MHz}, \mathrm{CDCl}_{3}\right) \delta(\mathrm{ppm})=145.7(\mathrm{Ar}), 138.0(\mathrm{C}-6), 135.7(\mathrm{Ar}), 134.7$ (C-2), 134.0 (C-10), 133.6 (Ar), 133.4 (Ar), 129.7 (Ar), 129.4 (Ar), 128.9 (Ar), 127.7 (Ar), 123.6 (C-7), 118.1 (C-3), 117.0 (C-11), 69.1 (C-1), 64.5 (C-8), 39.6 (C-5), 32.3 (C-9), 27.0 (TBDPS), 26.1 (C-4), 19.5 (TBDPS), 16.7 (Me), 13.7 (Me); HRMS [ESI] $\mathrm{m} / z$ calculated for $\mathrm{C}_{35} \mathrm{H}_{44} \mathrm{O}_{3} \mathrm{SiS}+\mathrm{Na}^{+}[\mathrm{M}+\mathrm{Na}]^{+}$ 595.2678, found 595.2676.

\section{$\boldsymbol{t}$-Butyl $\{[(2 E, 6 E)-2,6$-dimethylundeca-2,6,10-triene-1-yl]oxy\}diphenylsilane (S11)}

A suspension of sulfone $\mathbf{S 9}$ (0.53 g, $0.93 \mathrm{mmol}, 1.00$ equiv.) and $\mathrm{Pd}(\mathrm{dppe}) \mathrm{Cl}_{2}$ (26 mg, $0.05 \mathrm{mmol}, 0.05$ equiv.) in THF ( $8 \mathrm{~mL}$ ) was mixed dropwise with $\mathrm{LiBHEt}_{3}(1.7 \mathrm{M}$ in THF, $1.09 \mathrm{~mL}, 1.85 \mathrm{mmol}, 2.0$ equiv.) at $0{ }^{\circ} \mathrm{C}$ in a nitrogen atmosphere. The mixture was stirred for $3 \mathrm{~h}$ at this temperature and then mixed with $\mathrm{H}_{2} \mathrm{O}(15 \mathrm{~mL})$, brine $(30 \mathrm{~mL})$ and tert-butylmethyl ether $(30 \mathrm{~mL})$. The organic phase was separated and the aqueous phase extracted with tert-butylmethyl ether $(2 \times 30 \mathrm{~mL})$. The combined organic phases were dried over $\mathrm{MgSO}_{4}$, filtered and the solvent removed under reduced pressure. Column chromatographic purification on silica gel $(\mathrm{PE} / \mathrm{EtOAc}=100: 0 \rightarrow 50: 1)$ provided the title compound $\mathbf{S 1 1}(0.33 \mathrm{~g}, 0.76 \mathrm{mmol}, 81 \%)$ as a colourless oil.

${ }^{1} \mathbf{H}-N M R\left(400 \mathrm{MHz}, \mathrm{CDCl}_{3}\right): \delta(\mathrm{ppm})=7.72-7.67(\mathrm{~m}, 4 \mathrm{H}, \mathrm{Ar}), 7.45-7.35(\mathrm{~m}, 6 \mathrm{H}, \mathrm{Ar}), 5.88-5.77$ (m, $1 \mathrm{H}, 10-\mathrm{H}), 5.47-5.41(\mathrm{~m}, 1 \mathrm{H}, 3-\mathrm{H}), 5.19-5.13(\mathrm{~m}, 1 \mathrm{H}, 7-\mathrm{H}), 5.04-4.98\left(\mathrm{~m}, 1 \mathrm{H}, 11-\mathrm{H}_{\mathrm{b}}\right), 4.96-$ $4.92\left(\mathrm{~m}, 1 \mathrm{H}, 11-\mathrm{H}_{\mathrm{a}}\right), 4.05$ (s, $\left.2 \mathrm{H}, 1-\mathrm{H}\right), 2.18-2.00$ (m, 8 H, 4-H, 5-H, 8-H, 9-H), 1.63 (s, $\left.3 \mathrm{H}, 6-\mathrm{Me}\right)$, 1.61 (s, $3 \mathrm{H}, 2-\mathrm{Me}), 1.07$ (s, $9 \mathrm{H}, \mathrm{TBDPS})$; ${ }^{13} \mathrm{C}-\mathrm{NMR}\left(100 \mathrm{MHz}, \mathrm{CDCl}_{3}\right) \delta(\mathrm{ppm})=138.9(\mathrm{C}-10)$, 135.7 (Ar), 135.4 (Ar), 134.1 (C-6), 134.0 (C-2), 129.7 (Ar), 127.7 (Ar), 124.4 (C-7), 124.1 (C-3), 114.5 (C-11), 69.2 (C-1), 39.6 (C-5), 34.1 (C-9), 27.6 (C-8), 27.0 (TBDPS), 26.3 (C-4), 19.5 (TBDPS), 16.2 (6-Me), 13.7 (2-Me); HRMS [ESI] $\mathrm{m} / \mathrm{z}$ calculated for $\mathrm{C}_{29} \mathrm{H}_{40} \mathrm{OSi}+\mathrm{Na}^{+}[\mathrm{M}+\mathrm{Na}]^{+}$ 455.2746, found 455.2748 .

\section{(2E,6E)-2,6-Dimethylundeca-2,6,10-triene-1-ol (21)}

The silyl ether $\mathbf{S 1 0}(0.31 \mathrm{~g}, 0.71 \mathrm{mmol}, 1.0$ equiv.) in THF $(7 \mathrm{~mL})$ was mixed with TBAF (1 M in THF, $2.1 \mathrm{~mL}, 2.1 \mathrm{mmol}, 3.0$ equiv.) in a nitrogen atmosphere at ambient temperature and then stirred for $2 \mathrm{~h} . \mathrm{H}_{2} \mathrm{O}(30 \mathrm{~mL})$ and $\mathrm{Et}_{2} \mathrm{O}(30 \mathrm{~mL})$ were added, the organic phase was separated and the aqueous phase extracted with $\mathrm{Et}_{2} \mathrm{O}(2 \times 30 \mathrm{~mL})$. The combined organic phases were dried over $\mathrm{MgSO}_{4}$, filtered and the solvent was removed under reduced pressure. Column chromatographic purification on silica gel $(\mathrm{PE} / \mathrm{EtOAc}=100: 0 \rightarrow 25: 1 \rightarrow 20: 1)$ provided the title compound $\mathbf{2 1}(0.12 \mathrm{~g}, 0.63 \mathrm{mmol}, 89 \%)$ as a colourless oil. 
${ }^{1} \mathbf{H}-\mathbf{N M R}\left(400 \mathrm{MHz}, \mathrm{CDCl}_{3}\right): \delta(\mathrm{ppm})=5.88-5.76(\mathrm{~m}, 1 \mathrm{H}, 10-\mathrm{H}), 5.41-5.36(\mathrm{~m}, 1 \mathrm{H}, 3-\mathrm{H}), 5.17-$ $5.11(\mathrm{~m}, 1 \mathrm{H}, 7-\mathrm{H}), 5.04-4.98\left(\mathrm{~m}, 1 \mathrm{H}, 11-\mathrm{H}_{\mathrm{b}}\right), 4.97-4.92\left(\mathrm{~m}, 1 \mathrm{H}, 11-\mathrm{H}_{\mathrm{a}}\right), 3.99$ (s, $\left.2 \mathrm{H}, 1-\mathrm{H}\right), 2.18-$ 1.98 (m, 8 H, 4-H, 5-H, 8-H, 9-H), 1.66 (s, 3 H, 2-Me), 1.61 (s, 3 H, 6-Me); ${ }^{13}$ C-NMR (100 MHz, $\left.\mathrm{CDCl}_{3}\right) \delta(\mathrm{ppm})=138.9(\mathrm{C}-10), 135.2(\mathrm{C}-6), 134.9(\mathrm{C}-2), 126.2(\mathrm{C}-3), 124.2(\mathrm{C}-7), 114.6(\mathrm{C}-11), 69.2$ (C-1), 39.4 (C-5), 34.1 (C-9), 27.5 (C-8), 26.3 (C-4), 16.2 (6-Me), 13.8 (2-Me); HRMS [ESI] m/z calculated for $\mathrm{C}_{13} \mathrm{H}_{22} \mathrm{O}+\mathrm{Na}^{+}[\mathrm{M}+\mathrm{Na}]^{+} 217.1568$, found 217.1566 .

\section{Trisammonium (2E,6E)-2,6-Dimethylundeca-2,6,10-triene-1-yl-diphosphate (10)}

The synthesis was performed according to the general procedure for the synthesis of diphosphates starting from allyl alcohol 21 ( $0.30 \mathrm{~g}, 1.54 \mathrm{mmol}, 1.0$ equiv.). The title compound $\mathbf{1 0}(0.318 \mathrm{~g}, 0.785$ mmol, 51\%) was obtained as a colourless semisolid foam.

${ }^{1} \mathrm{H}-\mathrm{NMR}\left(400 \mathrm{MHz}, \mathrm{D}_{2} \mathrm{O}\right): \delta(\mathrm{ppm})=5.99-5.86(\mathrm{~m}, 1 \mathrm{H}, 10-\mathrm{H}), 5.60-5.53(\mathrm{~m}, 1 \mathrm{H}, 3-\mathrm{H}), 5.29-5.23$ $(\mathrm{m}, 1 \mathrm{H}, 7-\mathrm{H}), 5.11-5.05\left(\mathrm{~m}, 1 \mathrm{H}, 12-\mathrm{H}_{\mathrm{a}}\right), 5.03-4.98\left(\mathrm{~m}, 1 \mathrm{H}, 12-\mathrm{H}_{\mathrm{b}}\right), 4.32(\mathrm{~d}, J=5.4 \mathrm{~Hz}, 2 \mathrm{H}, 1-\mathrm{H})$, 2.25-2.04 (m, 8 H, 4-H, 5-H, 8-H, 9-H), 1.70 (s, 3 H, 2-Me), 1.64 (s, $3 \mathrm{H}, 6-\mathrm{Me}) ;{ }^{13} \mathrm{C}-\mathbf{N M R}$ $\left(100 \mathrm{MHz}, \mathrm{D}_{2} \mathrm{O}\right): \delta(\mathrm{ppm})=139.6(\mathrm{C}-10), 136.6(\mathrm{C}-6), 132.5$ (d, J = 8.4 Hz, C-2), $128.5(\mathrm{C}-3), 124.2$ (C-7), 114.4 (C-11), 71.4 (d, J = 5.4 Hz, C-1), 38.3 (C-5), 33.2 (C-9), 26.6 (C-8), 25.6 (C-4), 15.3 $(\mathrm{Me}), 13.0(\mathrm{Me}) ;{ }^{31} \mathrm{P}-\mathrm{NMR}\left(160 \mathrm{MHz}, \mathrm{D}_{2} \mathrm{O}\right): \delta(\mathrm{ppm})=-6.07(\mathrm{~d}, J=20.2 \mathrm{~Hz}),-10.3(\mathrm{~d}, J=20.4 \mathrm{~Hz})$;

\subsection{Synthesis of farnesylpyrophosphate derivative 11}

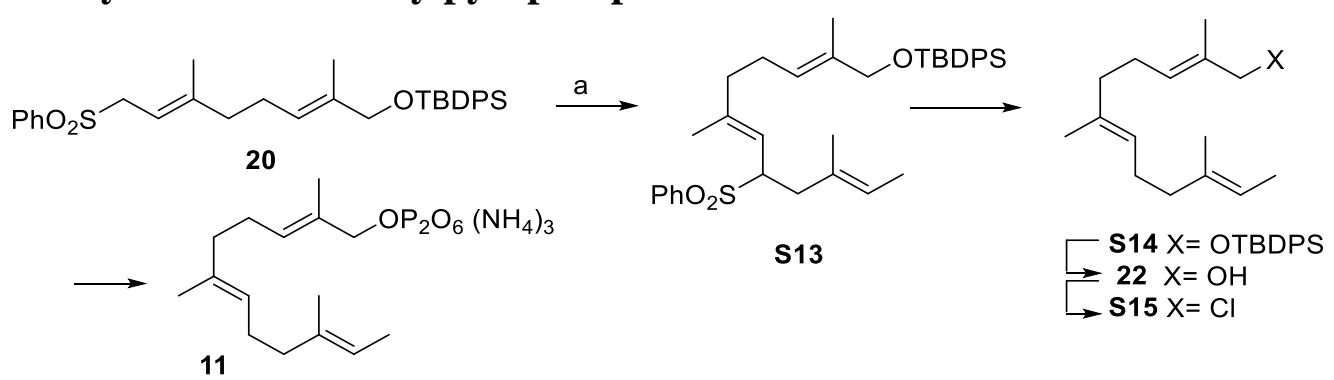

\section{$t$-Butyldiphenyl-\{[(2E,6E,10E)-2,6,10-trimethyl-8-(phenylsulfonyl)dodeca-2,6,10-triene-1- yl]oxy\}silane (S13)}

A solution of sulfone 20 (3.46 g, $6.50 \mathrm{mmol}, 1.0$ equiv.) in DME (20 mL) was mixed dropwise with KHMDS (0.5 M in toluene, $13.7 \mathrm{~mL}, 6.83 \mathrm{mmol}, 1.05$ equiv. $)$ at $-40{ }^{\circ} \mathrm{C}$ in a nitrogen atmosphere and then stirred for $0.5 \mathrm{~h}$. Then (E)-1-bromo-2-methylbut-2-ene (24b) (1.26 g, $8.45 \mathrm{mmol}, 1.30$ equiv.) was added dropwise and the mixture stirred for another $2 \mathrm{~h}$. A saturated aqueous $\mathrm{NH}_{4} \mathrm{Cl}$ solution (30 $\mathrm{mL}), \mathrm{H}_{2} \mathrm{O}(30 \mathrm{~mL})$ and tert-butylmethyl ether $(40 \mathrm{~mL})$ were added, the organic phase was separated and the aqueous phase was extracted with tert-butylmethyl ether $(2 \times 40 \mathrm{~mL})$. The combined organic phases were dried over $\mathrm{MgSO}_{4}$, filtered and the solvent removed under reduced pressure. Column chromatographic purification on silica gel $(\mathrm{PE} / \mathrm{EtOAc}=25: 1 \rightarrow 20: 1)$ provided title compound $\mathbf{S 1 3}$ (3.80 g, $6.33 \mathrm{mmol}, 97 \%)$ as a colourless oil.

${ }^{1} \mathbf{H}-N M R\left(400 \mathrm{MHz}, \mathrm{CDCl}_{3}\right): \delta(\mathrm{ppm})=7.86-7.83(\mathrm{~m}, 2 \mathrm{H}, \mathrm{Ar}), 7.69-7.65(\mathrm{~m}, 4 \mathrm{H}, \mathrm{Ar}), 7.62-7.58$ (m, $1 \mathrm{H}, \mathrm{Ar}), 7.53-7.47$ (m, $2 \mathrm{H}, \mathrm{Ar}), 7.44-7.35$ (m, $6 \mathrm{H}, \mathrm{Ar}), 5.35-5.29(\mathrm{~m}, 1 \mathrm{H}, 3-\mathrm{H})$, 5.24-5.17 (m, $1 \mathrm{H}, 11-\mathrm{H}), 4.94-4.89$ (m, $1 \mathrm{H}, 7-\mathrm{H}), 4.03$ (s, $2 \mathrm{H}, 1-\mathrm{H}), 3.88$ (td, J=10.6, $3.1 \mathrm{~Hz}, 1 \mathrm{H}, 8-\mathrm{H}), 2.91-$ $2.84\left(\mathrm{~m}, 1 \mathrm{H}, 9-\mathrm{H}_{\mathrm{a}}\right), 2.32-2.25\left(\mathrm{~m}, 1 \mathrm{H}, 9-\mathrm{H}_{\mathrm{b}}\right), 2.02-1.90(\mathrm{~m}, 4 \mathrm{H}, 4-\mathrm{H}, 5-\mathrm{H}), 1.59$ (s, $\left.3 \mathrm{H}, 2-\mathrm{Me}\right)$, 1.51-1.47 (m, $6 \mathrm{H}, 10-\mathrm{Me}, 11-\mathrm{Me}), 1.18$ (d, $J=1.3 \mathrm{~Hz}, 3 \mathrm{H}, 6-\mathrm{Me}), 1.06$ (s, $9 \mathrm{H}$, TBDPS); ${ }^{13} \mathrm{C}-\mathrm{NMR}$ $\left(100 \mathrm{MHz}, \mathrm{CDCl}_{3}\right) \delta(\mathrm{ppm})=145.1(\mathrm{Ar}), 138.2(\mathrm{C}-10), 135.7(\mathrm{Ar}), 134.7(\mathrm{C}-2), 134.0(\mathrm{Ar}), 133.5$ (Ar), 130.6 (C-6), 129.71 (Ar), 129.4 (Ar), 128.8 (Ar), 127.7 (Ar), 123.8 (C-3), 122.6 (C-11), 117.5 (C-7), 69.2 (C-1), 63.6 (C-8), 39.7 (C-5), 37.6 (C-9), 27.0 (TBDPS), 26.3 (C-4), 19.5 (TBDPS), 16.5 (6-Me), $15.7(\mathrm{Me}), 13.6(\mathrm{Me}), 13.6(\mathrm{Me})$; HRMS [ESI] $\mathrm{m} / z$ calculated for $\mathrm{C}_{37} \mathrm{H}_{48} \mathrm{O}_{3} \mathrm{SiS}+\mathrm{Na}^{+}$ $[\mathrm{M}+\mathrm{Na}]^{+}$623.2991, found 623.2992. 


\section{$t$-Butyldiphenyl $\{[(2 E, 6 E, 10 E)-2,6,10$-trimethyldodeca-2,6,10-triene-1-yl]oxy $\}$ silane (S14)}

A suspension of sulfone $\mathbf{S 1 1}\left(1.08 \mathrm{~g}, 1.79 \mathrm{mmol}, 1.00\right.$ equiv.) and $\mathrm{Pd}(\mathrm{dppe}) \mathrm{Cl}_{2}(51 \mathrm{mg}, 0.09 \mathrm{mmol}$, 0.05 equiv.) in THF (16 mL) was mixed dropwise with LiBHEt (1.7 M in THF, $2.1 \mathrm{~mL}, 3.58 \mathrm{mmol}$, 2.0 equiv.) at $0{ }^{\circ} \mathrm{C}$ in a nitrogen atmosphere. The mixture was stirred for $3 \mathrm{~h}$ at this temperature and then mixed with $\mathrm{H}_{2} \mathrm{O}(30 \mathrm{~mL})$, brine $(60 \mathrm{~mL})$ and tert-butylmethyl ether $(60 \mathrm{~mL})$. The organic phase was separated and the aqueous phase extracted with tert-butylmethyl ether $(2 \times 60 \mathrm{~mL})$. The combined organic phases were dried over $\mathrm{MgSO}_{4}$, filtered and the solvent removed under reduced pressure. Column chromatographic purification on silica gel $(\mathrm{PE} / \mathrm{EtOAc}=100: 0 \rightarrow 50: 1)$ provided the title compound $\mathbf{S 1 4}(0.39 \mathrm{~g}, 0.86 \mathrm{mmol}, 48 \%)$ as a colourless oil.

${ }^{1} \mathbf{H}-N M R\left(400 \mathrm{MHz}, \mathrm{CDCl}_{3}\right): \delta(\mathrm{ppm})=7.71-7.67(\mathrm{~m}, 4 \mathrm{H}, \mathrm{Ar}), 7.44-7.35(\mathrm{~m}, 6 \mathrm{H}, \mathrm{Ar}), 5.47-5.41$ (m, $1 \mathrm{H}, 3-\mathrm{H}), 5.24-5.17$ (m, $1 \mathrm{H}, 11-\mathrm{H}), 5.16-5.11(\mathrm{~m}, 1 \mathrm{H}, 7-\mathrm{H}), 4.05$ (s, $2 \mathrm{H}, 1-\mathrm{H}), 2.17-1.95$ (m, $8 \mathrm{H}, 4-\mathrm{H}, 5-\mathrm{H}, 8-\mathrm{H}, 9-\mathrm{H}), 1.63-1.55$ (m, $12 \mathrm{H}, 4 \times \mathrm{Me}$ ), 1.07 (s, $9 \mathrm{H}, \mathrm{TBDPS}) ;{ }^{13} \mathbf{C}-\mathbf{N M R}(100 \mathrm{MHz}$, $\left.\mathrm{CDCl}_{3}\right) \delta(\mathrm{ppm})=135.9(\mathrm{C}-10), 135.7(\mathrm{Ar}), 134.9(\mathrm{C}-6), 134.1(\mathrm{Ar}), 134.0(\mathrm{C}-2), 129.7$ (Ar), 127.7 (Ar), 124.6 (C-3/C-7), 124.4 (C-3/C-7), 118.4 (C-11), 69.2 (C-1), 39.9 (C-5/C-9), 39.6 (C-5/C-9), 27.0 (TBDPS), 26.8 (C-4/C-8), 26.3 (C-4/C-8), 19.5 (TBDPS), 16.1 (Me), 15.8 (Me), 13.7 (Me), 13.5 (Me); HRMS [ESI] $m / z$ calculated for $\mathrm{C}_{31} \mathrm{H}_{44} \mathrm{OSi}+\mathrm{Na}^{+}[\mathrm{M}+\mathrm{Na}]^{+} 483.3059$, found 483.3064 .

\section{(2E,6E,10E)-2,6,10-Trimethyldodeca-2,6,10-trien-1-ol (22)}

TBDPS-protected alcohol $\mathbf{S 1 4}(0.38 \mathrm{~g}, 0.81 \mathrm{mmol}, 1.0$ equiv.) in THF $(7 \mathrm{~mL})$ was mixed with TBAF (1 M in THF, $2.5 \mathrm{~mL}, 2.50 \mathrm{mmol}, 3.0$ equiv.) under a nitrogen atmosphere at ambient temperature and then stirred for $2.5 \mathrm{~h} . \mathrm{H}_{2} \mathrm{O}(30 \mathrm{~mL})$ and $\mathrm{Et}_{2} \mathrm{O}(30 \mathrm{~mL})$ were added, the organic phase was separated and the aqueous phase extracted with $\mathrm{Et}_{2} \mathrm{O}(2 \times 30 \mathrm{~mL})$. The combined organic phases were dried over $\mathrm{MgSO}_{4}$, filtered and the solvent removed under reduced pressure. Column chromatographic purification on silica gel $(\mathrm{PE} / \mathrm{EtOAc}=30: 1 \rightarrow 25: 1)$ provided the title compound $22(0.17 \mathrm{~g}, 0.77$ mmol, $94 \%)$ as a colourless oil.

${ }^{1} \mathbf{H}-\mathbf{N M R}\left(400 \mathrm{MHz}, \mathrm{CDCl}_{3}\right): \delta(\mathrm{ppm})=5.42-5.36(\mathrm{~m}, 1 \mathrm{H}, 3-\mathrm{H}), 5.24-5.17(\mathrm{~m}, 1 \mathrm{H}, 11-\mathrm{H}), 5.15-$ 5.09 (m, 1 H, 7-H), 3.99 (s, 2 H, 1-H), 2.16-1.96 (m, 8 H, 4-H, 5-H, 8-H, 9-H), 1.67 (s, 3 H, 2-Me), 2.61-1.59 (m, $6 \mathrm{H}, 6-\mathrm{Me}, 10-\mathrm{Me}), 1.58-1.55(\mathrm{~m}, 3 \mathrm{H}, 11-\mathrm{Me}) ;{ }^{13} \mathbf{C}-\mathbf{N M R}\left(100 \mathrm{MHz}, \mathrm{CDCl}_{3}\right) \delta$ $(\mathrm{ppm})=135.9(\mathrm{C}-10), 134.8(\mathrm{C}-6), 134.7(\mathrm{C}-2), 126.4(\mathrm{C}-3), 124.7$ (C-7), $118.5(\mathrm{C}-11), 69.2(\mathrm{C}-1)$, 39.8 (C-5/C-9), 39.4 (C-5/C-9), 26.8 (C-4/C-8), 26.4 (C-4/C-8), 16.1 (Me), 15.8 (Me), 13.8 (Me), 13.5 (Me); MS (EI): $m / z$ (rel. intensities): 204.2 (30) [M- $\left.\mathrm{H}_{2} \mathrm{O}\right], 189.2$ (29) [M- $\left.\mathrm{H}_{2} \mathrm{O}-\mathrm{Me}\right], 161.1$ (20) [M$\left.\mathrm{H}_{2} \mathrm{O}-\mathrm{C}_{3} \mathrm{H}_{7}\right], 107.1$ (100) $\left[\mathrm{C}_{8} \mathrm{H}_{11}\right], 93.1$ (100) $\left[\mathrm{C}_{7} \mathrm{H}_{9}\right], 69.1$ (34) $\left[\mathrm{C}_{5} \mathrm{H}_{9}\right], 55.1$ (38) $\left[\mathrm{C}_{4} \mathrm{H}_{7}\right], 41.0$ (40) $\left[\mathrm{C}_{3} \mathrm{H}_{5}\right]$; HRMS [ESI] $\mathrm{m} / \mathrm{z}$ calculated for $\mathrm{C}_{15} \mathrm{H}_{24}{ }^{+}\left[\mathrm{M}-\mathrm{H}_{2} \mathrm{O}\right]$ 204.1878, found 204.1882.

\section{Trisammonium (2E,6E,10E)-2,6,10-Trimethyldodeca-2,6,10-triene-1-yl-diphosphate (11)}

The synthesis was performed according to the general procedure for the synthesis of diphosphates starting from allyl alcohol 22 ( $0.30 \mathrm{~g}, 1.35 \mathrm{mmol}, 1.0$ equiv.). The title compound $\mathbf{1 1}(0.25 \mathrm{~g}, 0.59$ mmol, $43 \%$ ) was obtained as a colourless semisolid foam.

${ }^{1} \mathbf{H}-\mathrm{NMR}\left(400 \mathrm{MHz}, \mathrm{D}_{2} \mathrm{O}\right): \delta(\mathrm{ppm})=5.56-5.47(\mathrm{~m}, 1 \mathrm{H}, 3-\mathrm{H}), 5.23-5.09(\mathrm{~m}, 2 \mathrm{H}, 7-\mathrm{H}, 11-\mathrm{H}), 4.34-$ 4.28 (m, $2 \mathrm{H}, 1-\mathrm{H}), 2.13-1.93$ (m, $8 \mathrm{H}, 4-\mathrm{H}, 5-\mathrm{H}, 8-\mathrm{H}, 9-\mathrm{H}), 1.61-1.51$ (m, $12 \mathrm{H}, 4 \times \mathrm{Me}) ;{ }^{13} \mathrm{C}-\mathbf{N M R}$ $\left(100 \mathrm{MHz}, \mathrm{D}_{2} \mathrm{O}\right): \delta(\mathrm{ppm})=135.7(\mathrm{C}-6), 135.3(\mathrm{C}-10), 132.1(\mathrm{~d}, J=8.2 \mathrm{~Hz}, \mathrm{C}-2), 128.6(\mathrm{C}-3), 123.9$ (C-7), 118.2 (C-11), 72.1 (C-1), 39.5 (C-5/C-9), 38.9 (C-5/C-9), 26.5 (C-4/C-8), 26.5 (C-4/C-8), 15.7 (Me), $15.3(\mathrm{Me}), 13.1(\mathrm{Me}), 12.9(\mathrm{Me}) ;{ }^{31} \mathbf{P}-\mathbf{N M R}\left(160 \mathrm{MHz}, \mathrm{D}_{2} \mathrm{O}\right): \delta(\mathrm{ppm})=-10.01--11.31(\mathrm{~m}$, $2 \mathrm{P})$; HRMS [ESI] $m / z$ calculated for $\mathrm{C}_{15} \mathrm{H}_{27} \mathrm{O}_{7} \mathrm{P}_{2}\left[\mathrm{M}-3 \times \mathrm{NH}_{3}\right] 281.1232$, found 281.1231. 


\subsection{Synthesis of farnesylpyrophosphate derivative 12}

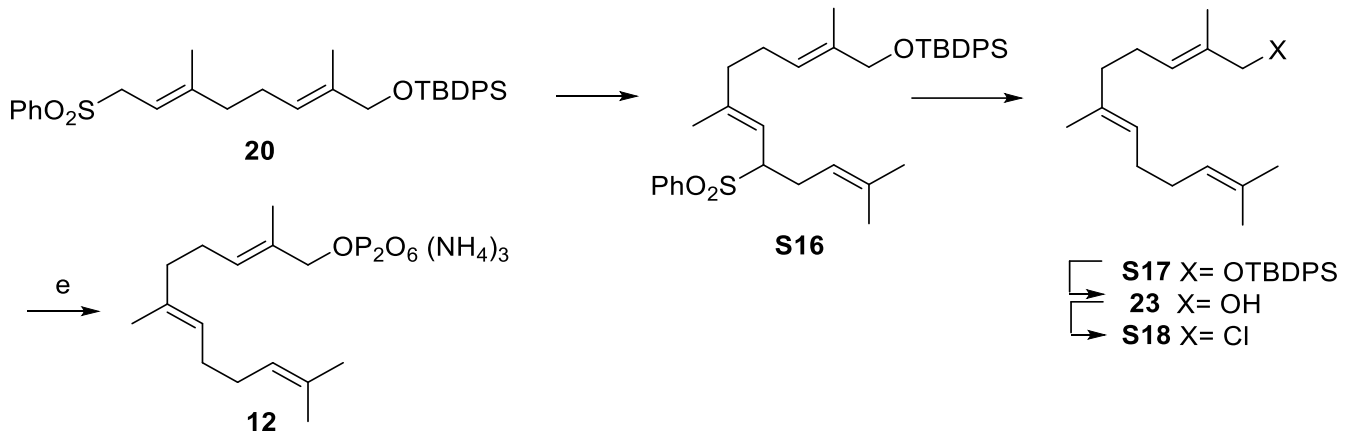

\section{$t$-Butyldiphenyl-\{[(2E,6E)-2,6,11-trimethyl-8-(phenylsulfonyl)dodeca-2,6,10-triene-1- yl]oxy\}silane (S16)}

A solution of sulfone 20 (3.46 g, $6.50 \mathrm{mmol}, 1.00$ equiv.) in DME (20 mL) was mixed dropwise with KHMDS (0.5 M in toluene, $13.7 \mathrm{~mL}, 6.83 \mathrm{mmol}, 1.05$ equiv.) at $-40{ }^{\circ} \mathrm{C}$ in a nitrogen atmosphere and then stirred for $0.5 \mathrm{~h}$. Then, 1-bromo-3-methylbut-2-ene $(\mathbf{2 4 c})(90 \%, 1.26 \mathrm{~mL}, 9.75 \mathrm{mmol}, 1.50$ equivalent) was added dropwise and the mixture was stirred for another $3 \mathrm{~h}$. A saturated aqueous $\mathrm{NH}_{4} \mathrm{Cl}$ solution $(30 \mathrm{~mL}), \mathrm{H}_{2} \mathrm{O}(30 \mathrm{~mL})$ and tert-butylmethyl ether $(40 \mathrm{~mL})$ were added, the organic phase was separated and the aqueous phase was extracted with tert-butylmethyl ether $(2 \times 40 \mathrm{~mL})$. The combined organic phases were dried over $\mathrm{MgSO}_{4}$, filtered and the solvent removed under reduced pressure. Column chromatographic purification on silica gel (PE/EtOAc $=25: 1 \rightarrow 20: 1)$ provided the title compound $\mathbf{S 1 6}$ (3.28 g, $5.46 \mathrm{mmol}, 84 \%)$ as a colourless oil.

${ }^{1} \mathbf{H}-N M R\left(400 \mathrm{MHz}, \mathrm{CDCl}_{3}\right): \delta(\mathrm{ppm})=7.87-7.82(\mathrm{~m}, 2 \mathrm{H}, \mathrm{Ar}), 7.70-7.65(\mathrm{~m}, 4 \mathrm{H}, \mathrm{Ar}), 7.62-7.57$ (m, $1 \mathrm{H}, \operatorname{Ar}), 7.53-7.47(\mathrm{~m}, 2 \mathrm{H}, \operatorname{Ar}), 7.45-7.34(\mathrm{~m}, 6 \mathrm{H}, \operatorname{Ar}), 5.37-5.31(\mathrm{~m}, 1 \mathrm{H}, 3-\mathrm{H}), 5.00(\mathrm{~d}$, $J=10.4 \mathrm{~Hz}, 1 \mathrm{H}, 7-\mathrm{H}), 4.97-4.91(\mathrm{~m}, 1 \mathrm{H}, 10-\mathrm{H}), 4.04(\mathrm{~s}, 2 \mathrm{H}, 1-\mathrm{H}), 3.73(\mathrm{td}, J=10.4,3.4 \mathrm{~Hz}, 1 \mathrm{H}$, 8-H), 2.91-2.82 (m, $\left.1 \mathrm{H}, 9-\mathrm{H}_{\mathrm{a}}\right), 2.39-2.29$ (m, $\left.1 \mathrm{H}, 9-\mathrm{H}_{\mathrm{b}}\right), 2.04-1.93$ (m, $\left.4 \mathrm{H}, 4-\mathrm{H}, 5-\mathrm{H}\right), 1.63$ (s, $3 \mathrm{H}$, 11-Me), 1.59 (s, $6 \mathrm{H}, 2-\mathrm{Me}, 11-\mathrm{Me}), 1.20$ (d, $J=1.1 \mathrm{~Hz}, 3 \mathrm{H}, 6-\mathrm{Me}), 1.06$ (s, $9 \mathrm{H}$, TBDPS); ${ }^{13} \mathbf{C}-\mathbf{N M R}$ $\left(100 \mathrm{MHz}, \mathrm{CDCl}_{3}\right) \delta(\mathrm{ppm})=145.3(\mathrm{Ar}), 138.3(\mathrm{C}-6), 135.7(\mathrm{Ar}), 135.1(\mathrm{C}-2), 134.7(\mathrm{C}-11), 134.0$ (Ar), 133.5 (Ar), 129.7 (Ar), 129.3 (Ar), 128.8 (Ar), 127.7 (Ar), 123.7 (C-10), 118.8 (C-7), 117.2 (C-3), 69.1 (C-1), 64.9 (C-8), 39.7 (C-5), 27.0 (TBDPS), 26.7 (C-9), 26.2 (C-4), 25.9 (Me), 19.5 (TBDPS), 18.2 (Me), 16.7 (Me), 13.7 (Me); HRMS [ESI] $\mathrm{m} / z$ calculated for $\mathrm{C}_{37} \mathrm{H}_{48} \mathrm{O}_{3} \mathrm{SiS}+\mathrm{Na}^{+}$ $[\mathrm{M}+\mathrm{Na}]^{+}$623.2991, found 623.2991.

\section{$t$-Butyldiphenyl-\{[(2E,6E)-2,6,11-trimethyldodeca-2,6,10-triene-1-yl]oxy\}silane (S17)}

A suspension of sulfone $\mathbf{S 1 3}$ (3.28 g, $5.46 \mathrm{mmol}, 1.0$ equiv.) and $\mathrm{Pd}(\mathrm{dppe}) \mathrm{Cl}_{2}(157 \mathrm{mg}, 0.27 \mathrm{mmol}$, 0.05 equiv.) in THF (30 mL) was mixed dropwise with $\mathrm{LiBHEt}_{3}(1.7 \mathrm{M}$ in THF, $10.9 \mathrm{~mL}, 10.9 \mathrm{mmol}$, 2.0 equiv.) at $0{ }^{\circ} \mathrm{C}$ in a nitrogen atmosphere. The mixture was stirred for $1.5 \mathrm{~h}$ at this temperature and then mixed with $\mathrm{H}_{2} \mathrm{O}(30 \mathrm{~mL})$, brine $(50 \mathrm{~mL})$ and tert-butylmethyl ether $(70 \mathrm{~mL})$. The organic phase was separated and the aqueous phase extracted with tert-butylmethyl ether $(2 \times 50 \mathrm{~mL})$. The combined organic phases were dried over $\mathrm{MgSO}_{4}$, filtered and the solvent was removed under reduced pressure. Column chromatographic purification on silica gel (PE/EtOAc $=100: 0 \rightarrow 80: 1)$ provided the title compound $\mathbf{S 1 7}$ (1.30 g, $2.83 \mathrm{mmol} ; 52 \%)$ as a colourless oil.

${ }^{1} \mathbf{H}-\mathbf{N M R}\left(400 \mathrm{MHz}, \mathrm{CDCl}_{3}\right): \delta(\mathrm{ppm})=7.71-7.67(\mathrm{~m}, 4 \mathrm{H}, \mathrm{Ar}), 7.45-7.35(\mathrm{~m}, 6 \mathrm{H}, \mathrm{Ar}), 5.47-5.41$ (m, 1 H, 3-H), 5.19-5.10 (m, 2 H, 7-H, 10-H), 4.05 (s, 2 H, 1-H), 2.18-1.98 (m, 8 H, 4-H, 5-H, 8-H, 9-H), 1.69 (s, $3 \mathrm{H}, \mathrm{Me}$ ), 1.62 (s, $3 \mathrm{H}, \mathrm{Me}$ ), 1.61 (s, $3 \mathrm{H}, \mathrm{Me}$ ), 1.60 (s, $3 \mathrm{H}, \mathrm{Me}$ ), 1.06 (s, $9 \mathrm{H}, \mathrm{TBDPS}$ ); ${ }^{13}$ C-NMR $\left(100 \mathrm{MHz}, \mathrm{CDCl}_{3}\right) \delta(\mathrm{ppm})=135.7$ (Ar), 135.1 (C-6), 134.1 (Ar), 134.0 (C-2), 131.6 (C-11), 129.7 (Ar), 127.7 (Ar), 124.6 (DB), 124.6 (DB), 124.5 (DB), 69.2 (C-1), 39.6 (C-5), 28.5 (C-8/C-9), 28.4 (C-8/C-9), 27.0 (TBDPS), 26.3 (C-4), 25.9 (Me), 19.5 (TBDPS), 17.9 (Me), 16.2 
(Me), 13.7 (Me); HRMS [ESI] $\mathrm{m} / z$ calculated for $\mathrm{C}_{31} \mathrm{H}_{44} \mathrm{OSi}+\mathrm{Na}^{+}[\mathrm{M}+\mathrm{Na}]^{+} 483.3059$, found 483.3067 .

\section{(2E,6E)-2,6,11-Trimethyldodeca-2,6,10-triene-1-ol (23)}

The TBDPS-protected alcohol S14 (1.30 g, $2.83 \mathrm{mmol}, 1.0$ equiv.) in THF (15 mL) was mixed with TBAF (1 $\mathrm{M}$ in THF, $8.5 \mathrm{~mL}, 8.50 \mathrm{mmol}, 3.0$ equiv.) under a nitrogen atmosphere at ambient temperature and then stirred for $3 \mathrm{~h} . \mathrm{H}_{2} \mathrm{O}(40 \mathrm{~mL})$ and $\mathrm{Et}_{2} \mathrm{O}(30 \mathrm{~mL})$ were added, the organic phase was separated and the aqueous phase extracted with $\mathrm{Et}_{2} \mathrm{O}(2 \times 30 \mathrm{~mL})$. The combined organic phases were dried over $\mathrm{MgSO}_{4}$, filtered and the solvent removed under reduced pressure. Column chromatographic purification on silica gel (PE/EtOAc $=30: 1 \rightarrow 20: 1)$ provided the title compound 23 $(0.58 \mathrm{~g}, 2.61 \mathrm{mmol}, 92 \%)$ as a colourless oil.

${ }^{1} \mathbf{H}-\mathbf{N M R}\left(400 \mathrm{MHz}, \mathrm{CDCl}_{3}\right): \delta(\mathrm{ppm})=5.42-5.37(\mathrm{~m}, 1 \mathrm{H}, 3-\mathrm{H}), 5.18-5.09(\mathrm{~m}, 2 \mathrm{H}, 7-\mathrm{H}, 10-\mathrm{H}), 3.99$ (s, $2 \mathrm{H}, 1-\mathrm{H}), 2.17-2.10$ (m, $2 \mathrm{H}, 4-\mathrm{H}), 2.05-1.98$ (m, $6 \mathrm{H}, 5-\mathrm{H}, 8-\mathrm{H}, 9-\mathrm{H}), 1.69$ (s, $3 \mathrm{H}, 11-\mathrm{Me}), 1.67$ (s, $3 \mathrm{H}, 2-\mathrm{Me}), 1.61-1.69$ (m, $6 \mathrm{H}, 6-\mathrm{Me}, 11-\mathrm{Me}) ;{ }^{13} \mathbf{C}-\mathbf{N M R}\left(100 \mathrm{MHz}, \mathrm{CDCl}_{3}\right) \delta(\mathrm{ppm})=134.9$ (C-6), 134.8 (C-2), 131.7 (C-11), 126.3 (C-3), 124.7 (C-7/C-10), 124.5 (C-7/C-10), 69.2 (C-1), 39.5 (C-5), 28.5 (C-8/C-9), 28.4 (C-8/C-9), 26.4 (C-4), 25.9 (11-Me), 17.9 (11-Me), 16.2 (6-Me), 13.8 (2-Me); MS (EI): $\mathrm{m} / z$ (rel. intensities): 204.2 (54) [M- $\left.\mathrm{H}_{2} \mathrm{O}\right], 189.2$ (54) $\left[\mathrm{M}-\mathrm{H}_{2} \mathrm{O}-\mathrm{Me}\right], 161.1$ (81) [M$\left.\mathrm{H}_{2} \mathrm{O}-\mathrm{C}_{3} \mathrm{H}_{7}\right], 135.1$ (57) $\left[\mathrm{C}_{10} \mathrm{H}_{15}\right], 107.1$ (96) $\left[\mathrm{C}_{8} \mathrm{H}_{11}\right], 93.1$ (100) $\left[\mathrm{C}_{7} \mathrm{H}_{9}\right], 69.1$ (90) $\left[\mathrm{C}_{5} \mathrm{H}_{9}\right], 55.1$ (48) $\left[\mathrm{C}_{4} \mathrm{H}_{7}\right], 41.0$ (62) $\left[\mathrm{C}_{3} \mathrm{H}_{5}\right]$; HRMS [ESI] $\mathrm{m} / z$ calculated for $\mathrm{C}_{15} \mathrm{H}_{24}{ }^{+}\left[\mathrm{M}-\mathrm{H}_{2} \mathrm{O}\right] 204.1878$, found 204.1879.

\section{Trisammonium (2E,6E)-2,6,11-Trimethyldodeca-2,6,10-triene-1-yl-diphosphate (12)}

The synthesis was performed according to the general procedure for the synthesis of diphosphates starting from allyl alcohol $\mathbf{2 3}$ ( $0.30 \mathrm{~g}, 1.35 \mathrm{mmol}, 1.00$ equiv.). The target compound $\mathbf{1 2}(0.34 \mathrm{~g}, 0.77$ mmol, $57 \%$ ) was obtained as a colourless semisolid foam.

${ }^{1} \mathbf{H}-\mathbf{N M R}\left(400 \mathrm{MHz}, \mathrm{D}_{2} \mathrm{O}\right): \delta(\mathrm{ppm})=5.54-5.47(\mathrm{~m}, 1 \mathrm{H}, 3-\mathrm{H}), 5.19-5.08(\mathrm{~m}, 2 \mathrm{H}, 7-\mathrm{H}, 10-\mathrm{H}), 4.31$ ( $\left.\mathrm{s}_{\mathrm{br}}, 2 \mathrm{H}, 1-\mathrm{H}\right), 2.10-1.93$ (m, $\left.8 \mathrm{H}, 4-\mathrm{H}, 5-\mathrm{H}, 8-\mathrm{H}, 9-\mathrm{H}\right), 1.67$ (s, $3 \mathrm{H}, \mathrm{Me}$ ), 1.65 (s, $3 \mathrm{H}, \mathrm{Me}$ ), 1.59 (s, $3 \mathrm{H}, \mathrm{Me}), 1.58(\mathrm{~s}, 3 \mathrm{H}, \mathrm{Me}) ;{ }^{13} \mathrm{C}-\mathrm{NMR}\left(100 \mathrm{MHz}, \mathrm{D}_{2} \mathrm{O}\right): \delta(\mathrm{ppm})=135.5(\mathrm{C}-6), 132.1(\mathrm{~d}, J=8.1 \mathrm{~Hz}$, C-2), 131.2 (C-3), 128.6 (C-11), 124.5 (C-7), 123.9 (C-10), 72.0 (d, J=4.8 Hz, C-1), 38.9 (C-5), 28.2 (C-8/C-9), 28.1 (C-8/C-9), 26.5 (C-4), 25.3 (Me), 17.3 (Me), 15.7 (Me), 13.1 (Me); ${ }^{31} \mathbf{P}-\mathbf{N M R}$ $\left(160 \mathrm{MHz}, \mathrm{D}_{2} \mathrm{O}\right): \delta(\mathrm{ppm})=-9.98--11.52(\mathrm{~m}, 2 \mathrm{P}) ;$ HRMS $[\mathrm{ESI}] \mathrm{m} / z$ calculated for $\mathrm{C}_{15} \mathrm{H}_{27} \mathrm{O}_{7} \mathrm{P}_{2}[\mathrm{M}-$ $\left.3 \times \mathrm{NH}_{3}\right]$ 281.1232, found 281.1234.

\section{Optimisation of biotransformations with FPP (17) as substrate}

All optimisations with $\mathrm{BcBOT} 2$ were performed in triplicate with $\beta$-caryophyllene, respectively, as internal standards for semi-quantification. First, a broad optimisation was carried out with FPP (17) as test substrate. The optimisation was directed towards improving the yield of the major cyclisation product. Next, selected biotransformations were optimised with unnatural substrates with respect to the role of the temperature for semi-preparative scale.

The extraction procedure was optimised with respect to time, concentration and number of extraction steps. FPP $(150 \mu \mathrm{M})$ was used as test substrate and BcBOT2 $(0.01 \mathrm{~g} / \mathrm{L})$ as test enzyme. The yield of presilphiperfolan- $8 \beta$-ol was determined by semi-quantitative evaluation using $\beta$-caryophyllene as internal standard. At least $100 \mu \mathrm{L}$ of $n$-hexane are required for each extraction process. A one-time extraction and $30 \mathrm{~s}$ extraction time the product could be fully transferred into the organic phase. Longer extraction times and larger volumes did not result in higher yields. Furthermore, multi-step extraction showed that after the second extraction step no additional product could be collected. 


\subsection{Temperature and $\mathrm{pH}$}

The temperature was optimised probing a range between $5{ }^{\circ} \mathrm{C}$ and $40{ }^{\circ} \mathrm{C}$ (Thermo Mixer). Here, BcBOT2 showed highest activity between $20^{\circ} \mathrm{C}$ to $25^{\circ} \mathrm{C}$.

Figure S2: semi-quantitative determination of PSP depending on the temperature

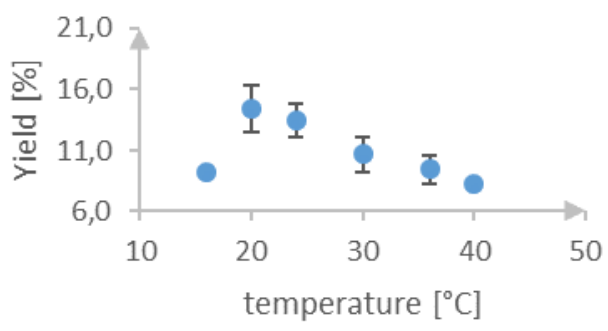

The $\mathrm{pH}$ was optimised between $\mathrm{pH} 3$ and 11 (table $\mathrm{S} 3)$. For BcBOT2 a broad optimum between $\mathrm{pH} 6$ 9 was acceptable.

Table S3. Buffers and $\mathrm{pH}$ values probed and semi-quantification of PSP-production depending on $\mathrm{pH}$ value.

\begin{tabular}{ll}
\hline pH & buffer [conc.] \\
\hline 3.1 & citric acid/ $/ \mathrm{Na}_{2} \mathrm{HPO}_{4}(0.1 \mathrm{M} / 0.2 \mathrm{M})$ \\
4.1 & citric acid/Na $\mathrm{HPO}_{4}(0.1 \mathrm{M} / 0.2 \mathrm{M})$ \\
5.0 & citric acid/ $/ \mathrm{Na}_{2} \mathrm{HPO}_{4}(0.1 \mathrm{M} / 0.2 \mathrm{M})$ \\
6.0 & MES $(50 \mathrm{mM})$ \\
7.0 & HEPES $(50 \mathrm{mM})$ \\
8.0 & HEPES $(50 \mathrm{mM})$ \\
9.2 & Tris-HCl $(50 \mathrm{mM})$ \\
10.0 & CAPS $(50 \mathrm{mM})$ \\
11.0 & CAPS $(50 \mathrm{mM})$ \\
\hline
\end{tabular}

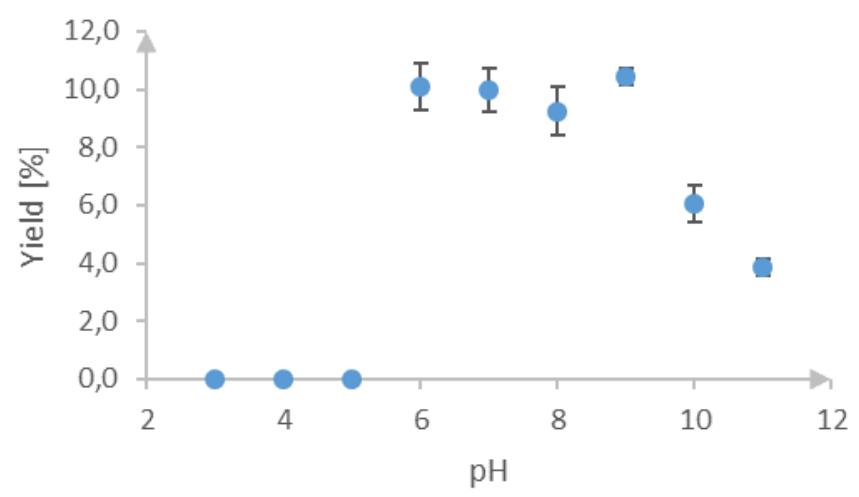

\subsection{Concentration of enzyme, substrate and $\mathrm{Mg}^{2+}$}

For determining optimal enzyme and substrate concentrations, analytical assays (concentration range between $0.005 \mathrm{~g} \cdot \mathrm{L}^{-1}$ and $0.2 \mathrm{~g} \cdot \mathrm{L}^{-1}$ for the terpene cyclase and $5 \mu \mathrm{M}$ to $150 \mu \mathrm{M}$ for FPP) were carried out. For BcBOT2 substrate inhibition was observed. Nevertheless, for BcBOT2 the optimal concentration was found to be $0.01 \mathrm{~g} \cdot \mathrm{L}^{-1}$ and $20 \mu \mathrm{M}$ for FPP. 


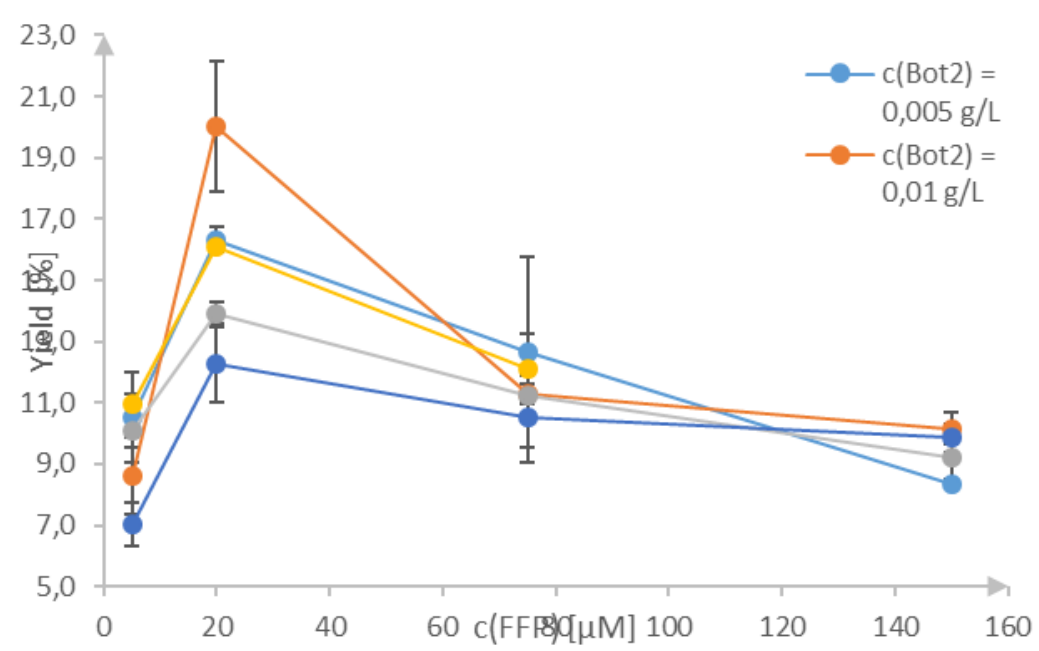

Figure S3: Production of PSP vary in relation to enzyme concentration and substrate concentration. The lines between each meaurement point are only shown for better clarity.

Also, the influence of the $\mathrm{MgCl}_{2}$ concentration (between $0.5 \mathrm{mM}$ and $50 \mathrm{mM}$ ) on substrate solubility and enzyme activity was determined. For Bot 2 a range between $5 \mathrm{mM}$ and $10 \mathrm{mM}$ is acceptable. Lower concentrations led to incomplete activation of the enzymes while higher concentrations might favour precipitation of the the pyrophosphate.

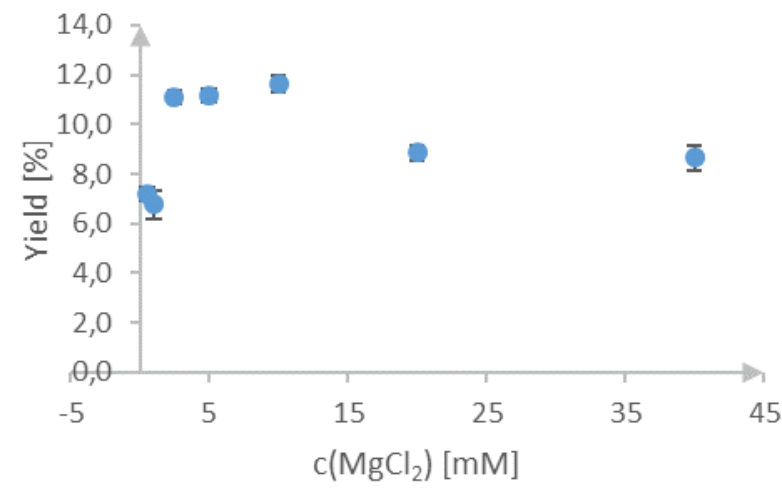

Figure S4: Production of PSP in realtion to concentration of cofactor $\mathrm{MgCl}_{2}$

\subsection{Unnatural substrates}

Finally, the influence of the temperature on the enzymatic conversion of the methylshifted non-natural pyrophosphates was optimised in order to carry out biotransformations in preparative scale. Therefore, analytical assays $\left(0.01 \mathrm{~g} / \mathrm{L} \mathrm{BcBOT} 2,150 \mu \mathrm{M}\right.$ non-natural substrate, $5 \mu \mathrm{M} \mathrm{MgCl}_{2}$, conditions: $20 \mathrm{~min}$, $0 \mathrm{rpm}$ ) were done on triplicate with non-natural substrates 9, 11 and 12 and BcBOT2 at different temepratures $\left(10^{\circ} \mathrm{C}-40^{\circ} \mathrm{C}\right)$. For semi-quantitative evaluation an internal standard ( $\beta$-Caryophyllene) was used, respectively. We found that in all cases the optimal temperature was shifted to higher temperatures (see figure 2). Furthermore, Proteinase K (from Tritirachium album) for enzyme degradation, Tween 20 als solubiliser and PPase (from S. cerevisiae) for degradation of the resulting diphosphates were tested on analytical scale to analyse potential side effects for semi-preparative scale. 
Figure S5. Optimisation of biotransformation conditions (triplicate) for BcBOT2 with FPP 1 and $\mathbf{9 , 1 1}$ and 12 as substrates (internal standards: $\beta$-caryophyllene and $\alpha$-humulene depending on retention times of all products detected).
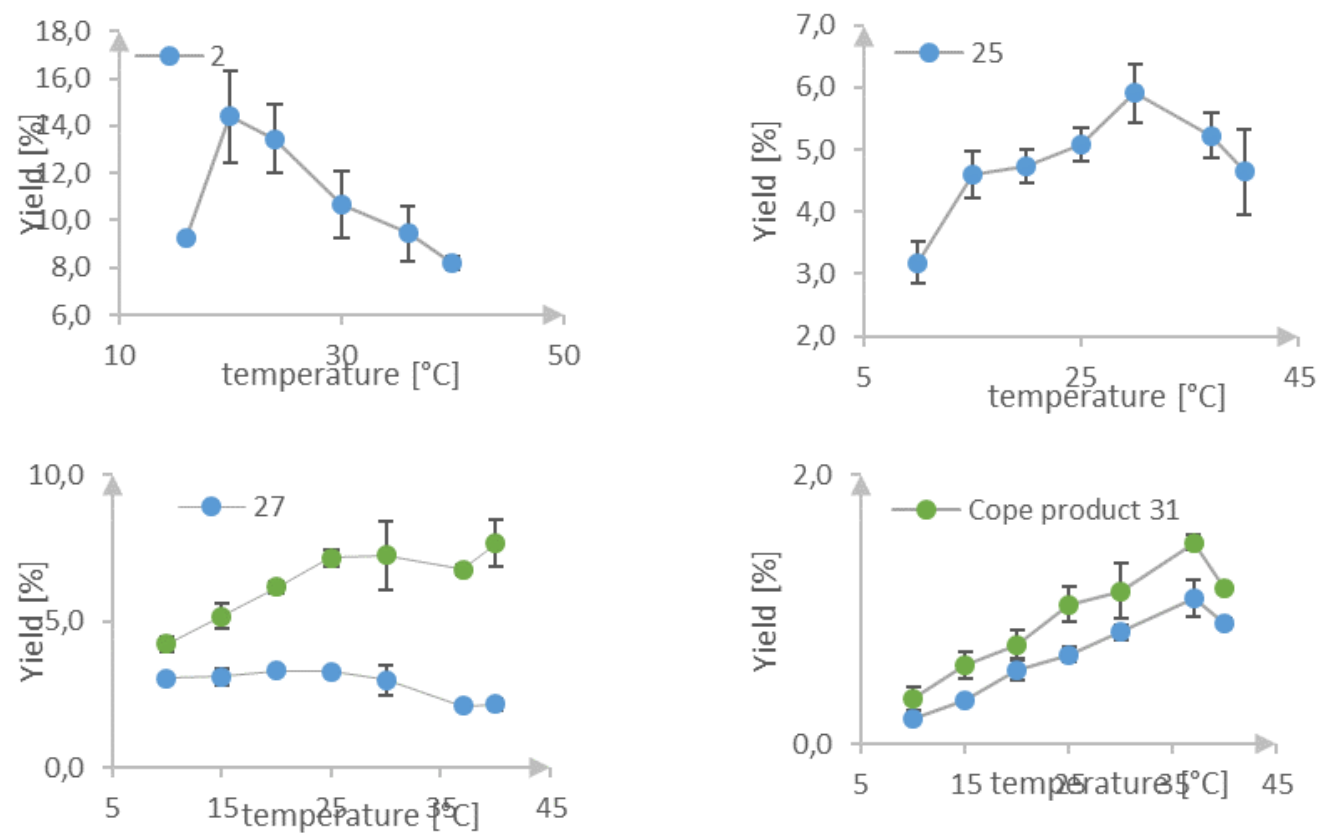

\section{Biotransformations with unnatural farnesyl pyrophosphates on semi-preparative scale}

\section{1 general procedure for the enzymatic conversion of Bot2 with 9, 11 and 12}

Semi preparative assays with $1.5 \mathrm{mM}$ substrate were performed in HEPES buffer $(50 \mathrm{mM}, \mathrm{pH} 7.5)$ supplemented with DTT $(5 \mathrm{mM})$ and $\mathrm{MgCl}_{2}$. The enzyme BcBOT2 was purified by IMAC as described above, transferred to HEPES buffer and utilised in a concentration of $0.1 \mathrm{~g} \cdot \mathrm{L}^{-1}$. Tween20 and pyrophosphatase (from S. cerevisiae) were added to prevent substrate precipitation or enzyme inhibition by inorganic diphosphate. The mixture was incubated at optimal temperature and rpm. Then, $\mathrm{CaCl}_{2}$ and Proteinase $\mathrm{K}$ were added for protein degradation during further incubation. The mixture was overlayed with n-pentane at $16^{\circ} \mathrm{C}$ and $110 \mathrm{rpm}$ overnight. Afterwards the layers were separated and the watery phase was again extracted triplicate with $n$-pentane. The combined organic phase was washed with brine, dried with $\mathrm{MgSO}_{4}$ and filtered. After removal of the solvent, yellowish and wax-like substances 25, 26, 27 and 28 were collected.

\subsubsection{Defined parameters}

Table S4: Parameters for the biotransformation of Bot2 with non-natural substrates $\mathbf{9 ,}, \mathbf{1 1}$ and $\mathbf{1 2 .}$

\begin{tabular}{|c|c|c|c|}
\hline & 9 & 11 & 12 \\
\hline c(substrate) $[\mathrm{mM}]$ & 1.5 & 1.5 & 1.5 \\
\hline $\mathbf{c}\left(\mathbf{M g C l}_{2}\right)[\mathrm{mM}]$ & 25.0 & 25.0 & 25.0 \\
\hline$\beta(B o t 2)\left[g \cdot L^{-1}\right]$ & 0.1 & 0.1 & 0.1 \\
\hline PPase [U] & 1.0 & 1.0 & 1.0 \\
\hline $\mathrm{V}$ (assay) [mL] & 46.1 & 46.1 & 44.5 \\
\hline duration [h] & 5.5 & 5.5 & 5.75 \\
\hline temperature $\left[{ }^{\circ} \mathbf{C}\right]$ & 30 & 25 & 28 \\
\hline rotation [rpm] & 180 & 180 & 180 \\
\hline $\mathrm{CaCl}_{2}[\mathrm{mM}]$ & 5.0 & 5.0 & 5.0 \\
\hline Proteinase K [U] & 75 & 75 & 75 \\
\hline duration [h] & 1.0 & 1.0 & 1.0 \\
\hline temperature $\left[{ }^{\circ} \mathbf{C}\right]$ & 28 & 25 & 25 \\
\hline rotation $[\mathrm{rpm}]$ & 180 & 180 & 180 \\
\hline
\end{tabular}




\subsection{Structure elucidation of 25}

Product 25 was isolated from the biotransformation of Bot 2 with non-natural substrate 9 in $12.8 \%$ yield. Purification was done by pGC. The purified product $(1 \mathrm{mg})$ was solved in $\mathrm{C}_{6} \mathrm{D}_{6}$.

HR-GC-MS: exact mass: 204.1878, found: 204.1877

RI $\mathrm{DB}_{\mathrm{DB}}: 1518$

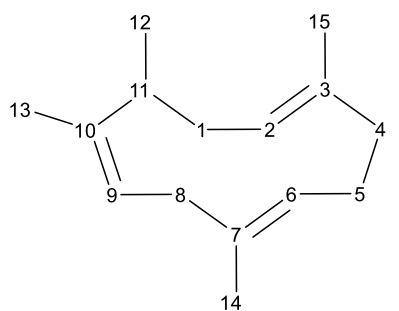

\begin{tabular}{|c|c|c|c|c|c|c|c|c|}
\hline $\mathrm{Nr}$ & $\delta^{13 C}$ & $\delta^{1 \mathrm{H}}$ & $\# \mathbf{H}$ & Multi & $\mathrm{J}(\mathrm{Hz})$ & cosy & HMBC & 1D-noe \\
\hline $\begin{array}{l}1 a \\
1 b\end{array}$ & 33.0 & $\begin{array}{l}1.90 \\
2.14\end{array}$ & 2 & $\begin{array}{c}d d \\
d d d\end{array}$ & $\begin{array}{c}5.5,10.2 \\
5.5,9.6,10.2\end{array}$ & $\begin{array}{c}1 b, 11,2 \\
1 a, 11,2\end{array}$ & 12 & $1 b, 2,12,13$ \\
\hline 2 & 126.7 & 4.97 & 1 & dd & $5.5,11.1$ & $1 a, 1 b, 15$ & $\begin{array}{c}1 a, 1 b, 4 a \\
4 b, 15\end{array}$ & $5 a, 12,14$ \\
\hline 3 & 133.5 & & & & & & $\begin{array}{l}1 a, 1 b, 4 a, \\
4 b, 5 a, 15\end{array}$ & - \\
\hline $\begin{array}{l}4 a \\
4 b\end{array}$ & 39.4 & $\begin{array}{l}2.01 \\
2.09\end{array}$ & 2 & $\begin{array}{l}d t \\
d d\end{array}$ & $\begin{array}{l}7.1,10.4 \\
7.1,10.4\end{array}$ & $\begin{array}{l}4 b \\
4 a\end{array}$ & $1 b, 2,15$ & - \\
\hline $\begin{array}{l}5 a \\
5 b\end{array}$ & 24.1 & $\begin{array}{l}2.03 \\
2.11\end{array}$ & 2 & $\begin{array}{l}\mathrm{dt} \\
\mathrm{m}\end{array}$ & $7.1,14.0$ & $\begin{array}{c}5 b, 15 \\
5 a, 6\end{array}$ & $4 a, 4 b$ & - \\
\hline 6 & 126.0 & 4.95 & 1 & $\mathrm{~m}$ & & $5 b, 14$ & $\begin{array}{c}5 b, 8 a, 8 b, \\
14\end{array}$ & $\begin{array}{c}4 a, 5 b, 8 b \\
9,11\end{array}$ \\
\hline 7 & 134.4 & & & & & & $\begin{array}{c}8 a, 8 b, 13, \\
14\end{array}$ & \\
\hline $\begin{array}{l}8 a \\
8 b\end{array}$ & 36.2 & $\begin{array}{l}2.50 \\
2.63\end{array}$ & 2 & $\begin{array}{l}d d \\
d d\end{array}$ & $\begin{array}{l}7.9,16.0 \\
7.9,16.0\end{array}$ & $\begin{array}{l}9,14 \\
9,13\end{array}$ & $9,13,14$ & $\begin{array}{c}8 b, 9,14 \\
8 a, 9,11,14\end{array}$ \\
\hline 9 & 123.3 & 5.47 & 1 & $\mathrm{td}$ & $1.4,7.9$ & $8 a, 8 b, 13$ & $8 a, 8 b, 13$ & $6,8 a, 8 b, 13$ \\
\hline 10 & 141.5 & & & & & & $\begin{array}{l}1 a, 1 b, 8 a, \\
8 b, 12,13\end{array}$ & - \\
\hline 11 & 36.2 & 2.42 & 1 & $\mathrm{~m}$ & & $1 a, 1 b, 12$ & 1a, 1b, 12 & $6,8 b, 12,15$ \\
\hline 12 & 20.0 & 0.95 & 3 & $d$ & 6.9 & 11 & 11 & $\begin{array}{c}1 a, 1 b, 11 \\
13\end{array}$ \\
\hline 13 & 17.5 & 1.63 & 3 & d & 1.0 & $8 b, 9$ & 9,11 & $1 a, 2,9,12$ \\
\hline 14 & 17.0 & 1.51 & 3 & $d$ & 0.9 & $8 a, 5 a, 6$ & $8 b$ & $\begin{array}{l}2,5 a, 5 b \\
8 a, 8 b, 9\end{array}$ \\
\hline 15 & 14.7 & 1.41 & 3 & $t$ & 0.8 & $2,5 b$ & $5 a, 4 b, 2$ & $1 b, 6,11$ \\
\hline
\end{tabular}


${ }^{1} \mathrm{H}-\mathrm{NMR}(\mathbf{2 5})-500 \mathrm{MHz}, \mathrm{C}_{6} \mathrm{D}_{6}$

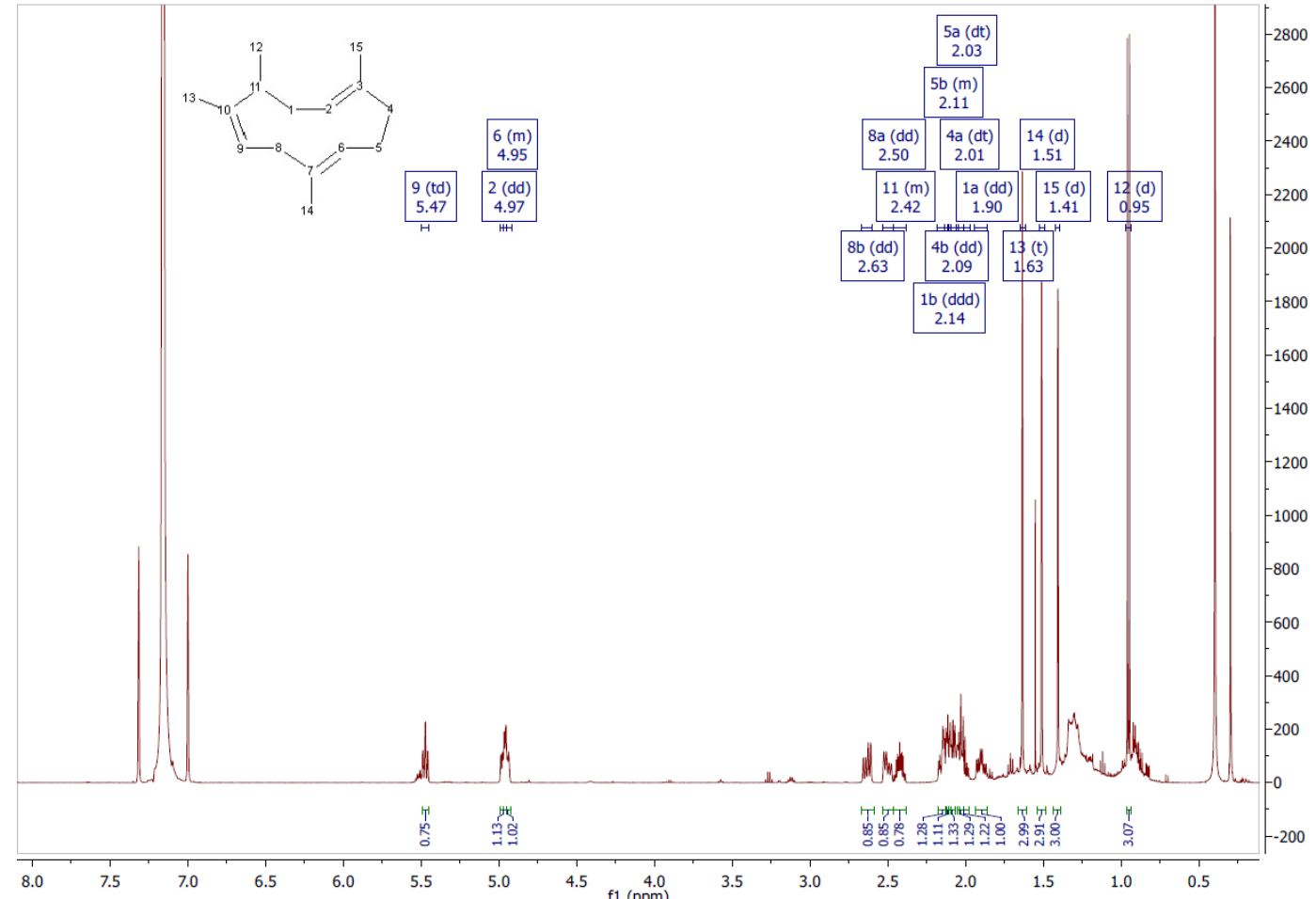

${ }^{13} \mathrm{C}-\mathrm{NMR}(\mathbf{2 5})-126 \mathrm{MHz}, \mathrm{C}_{6} \mathrm{D}_{6}$

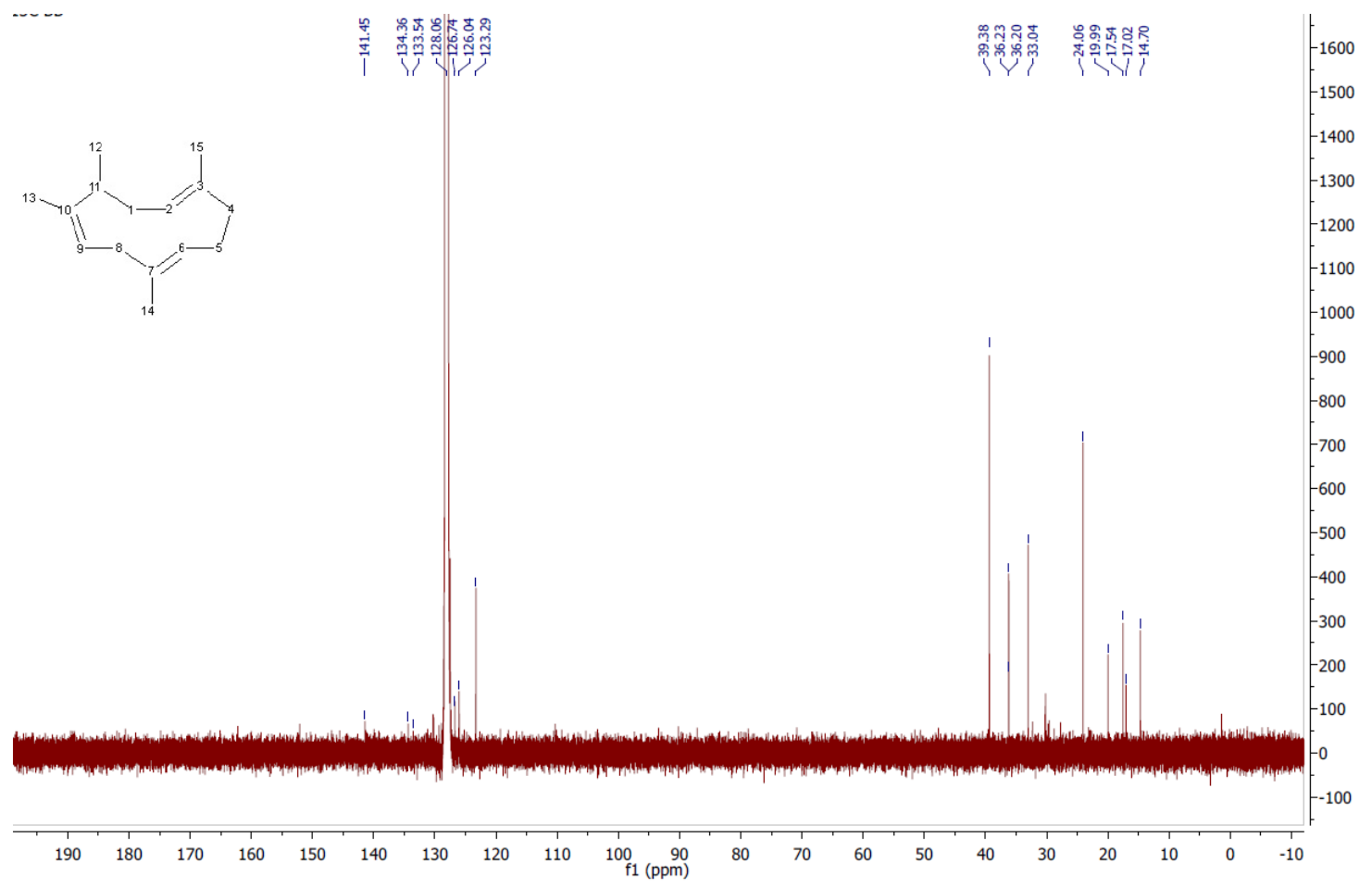


DEPT135 (25) - $126 \mathrm{MHz}, \mathrm{C}_{6} \mathrm{D}_{6}$

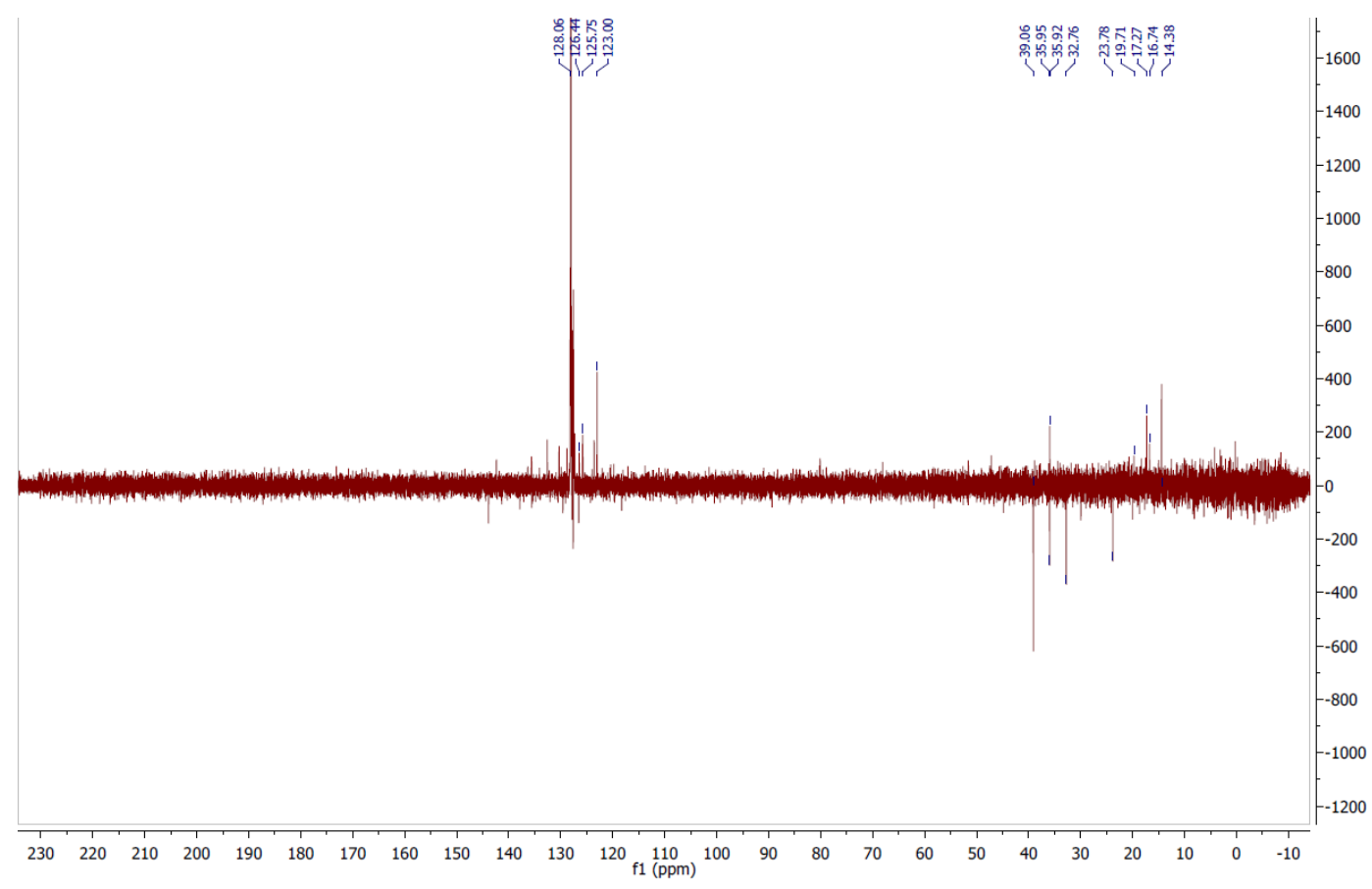

HSQC(25) - $500 \mathrm{MHz}, \mathrm{C}_{6} \mathrm{D}_{6}$

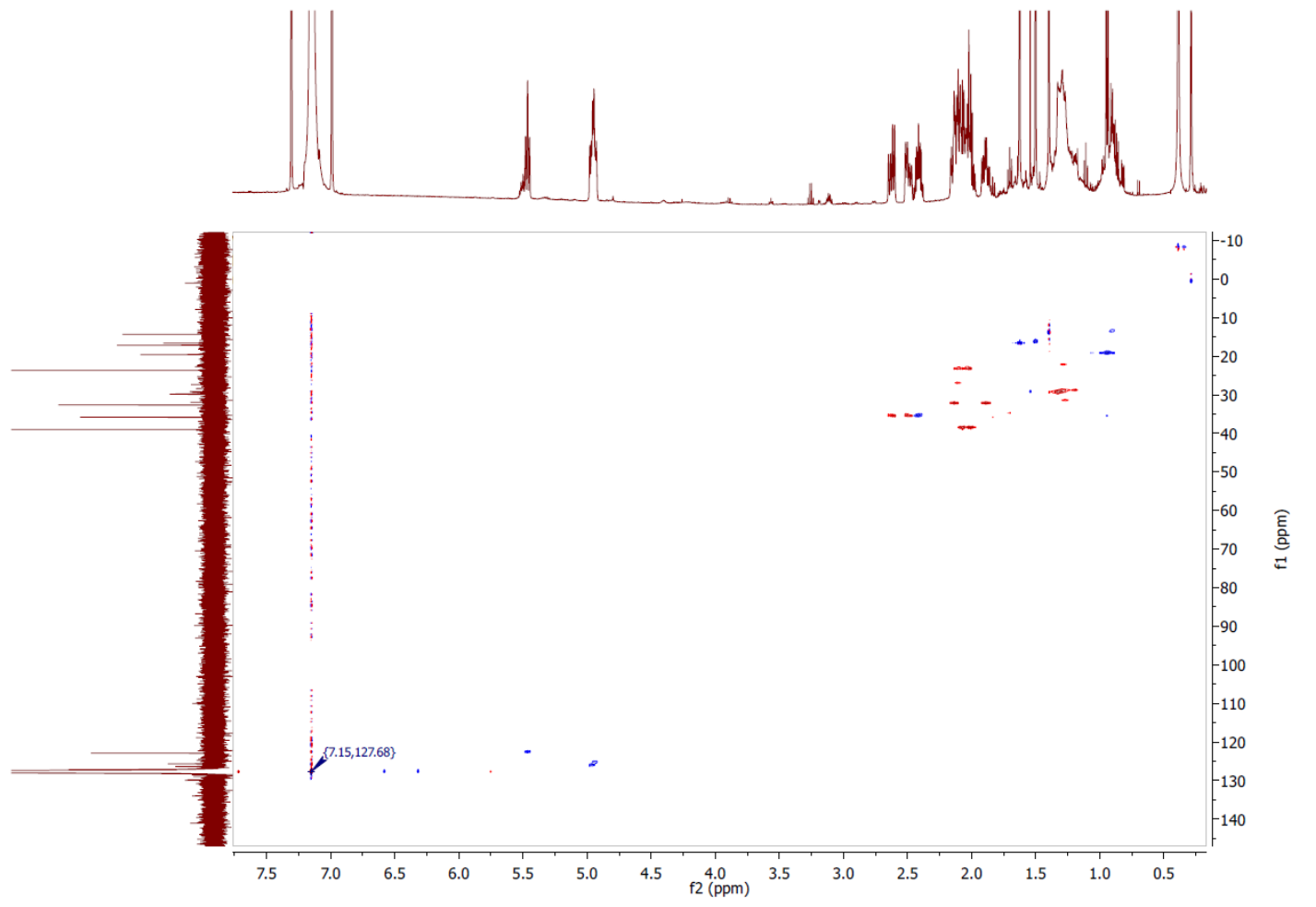


23

COSY (25) - $500 \mathrm{MHz}, \mathrm{C}_{6} \mathrm{D}_{6}$

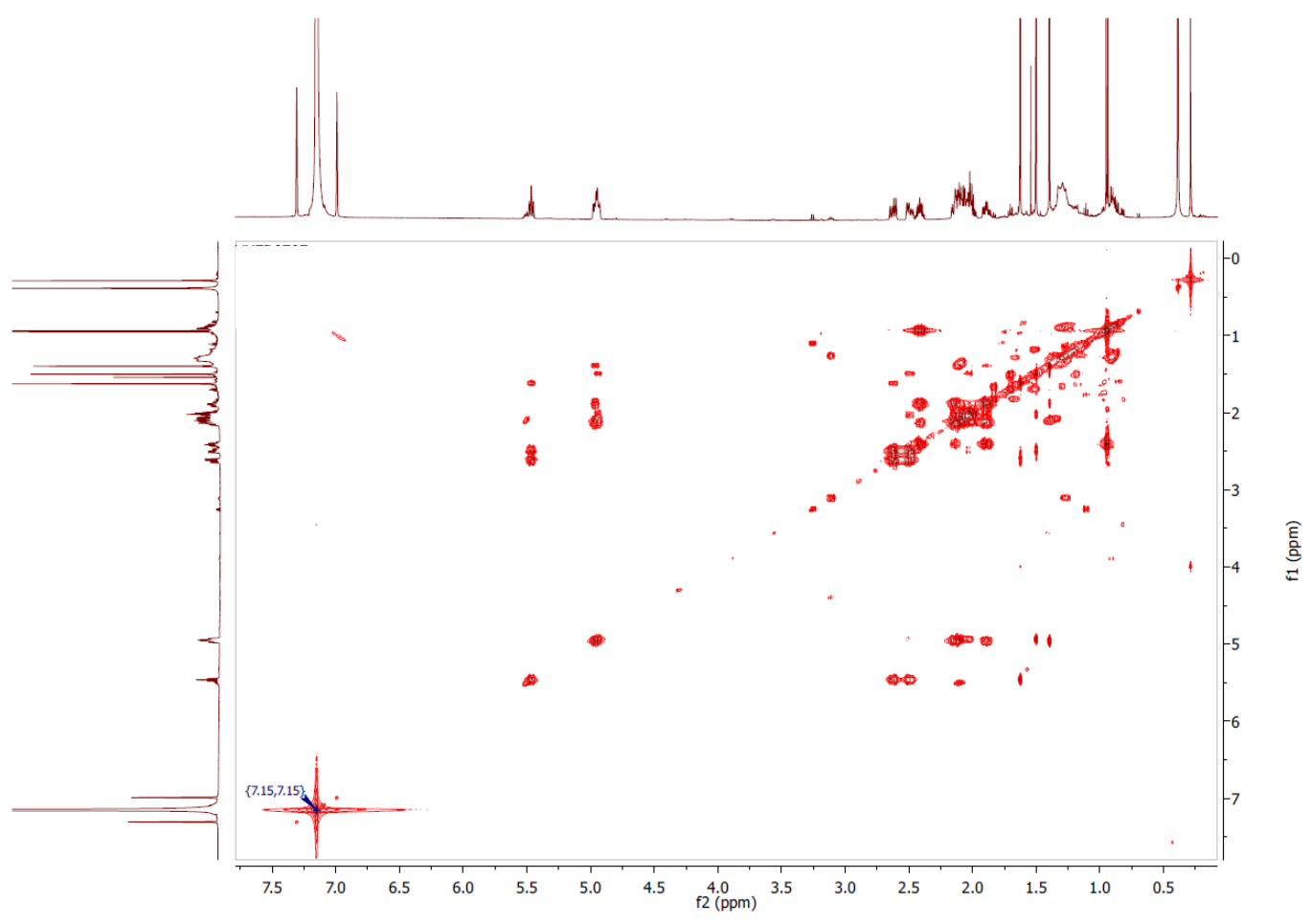

HMBC (25) - 500 MHz, $\mathrm{C}_{6} \mathrm{D}_{6}$

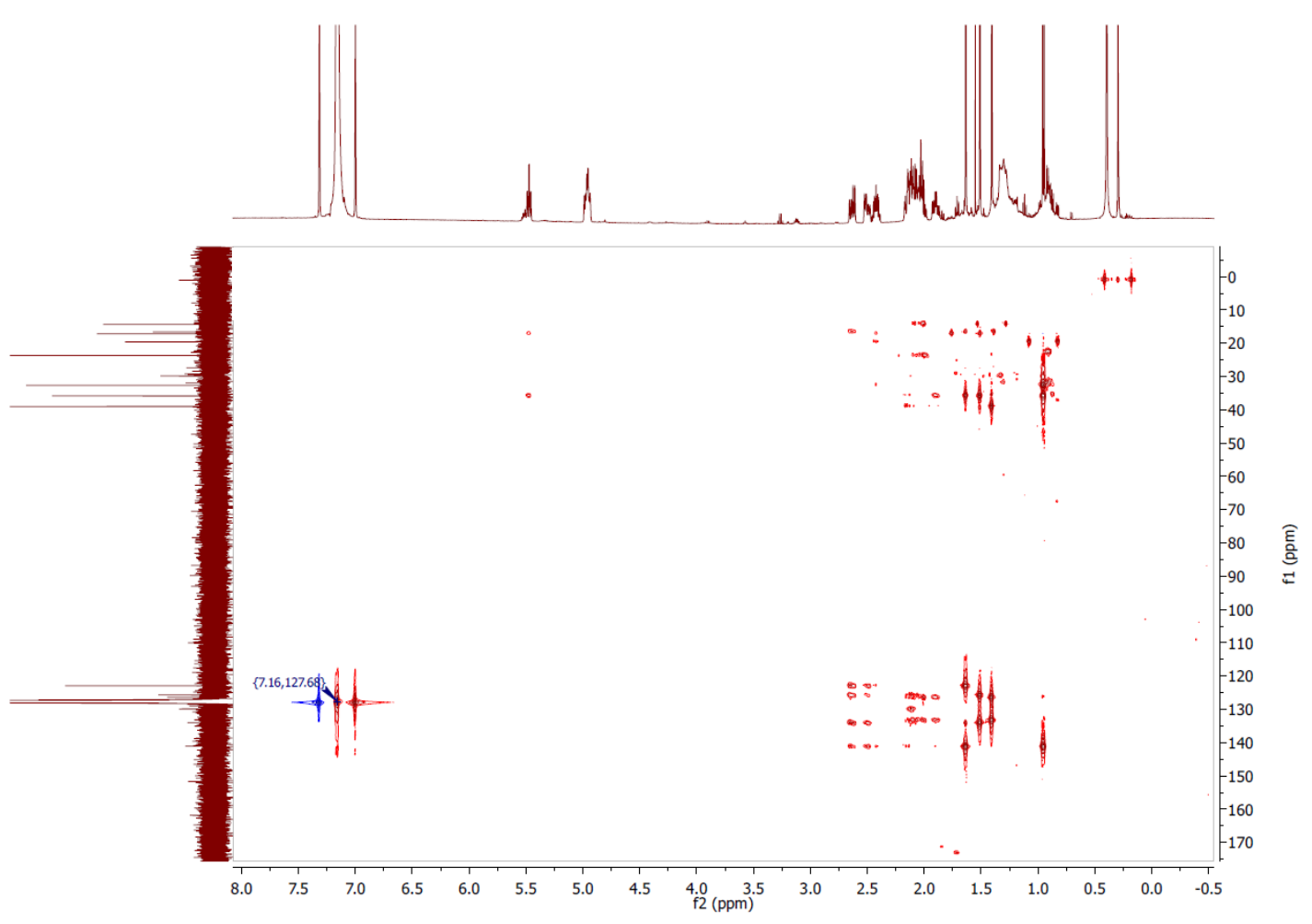


1d-NOE (25) - $500 \mathrm{MHz}, \mathrm{C}_{6} \mathrm{D}_{6}$
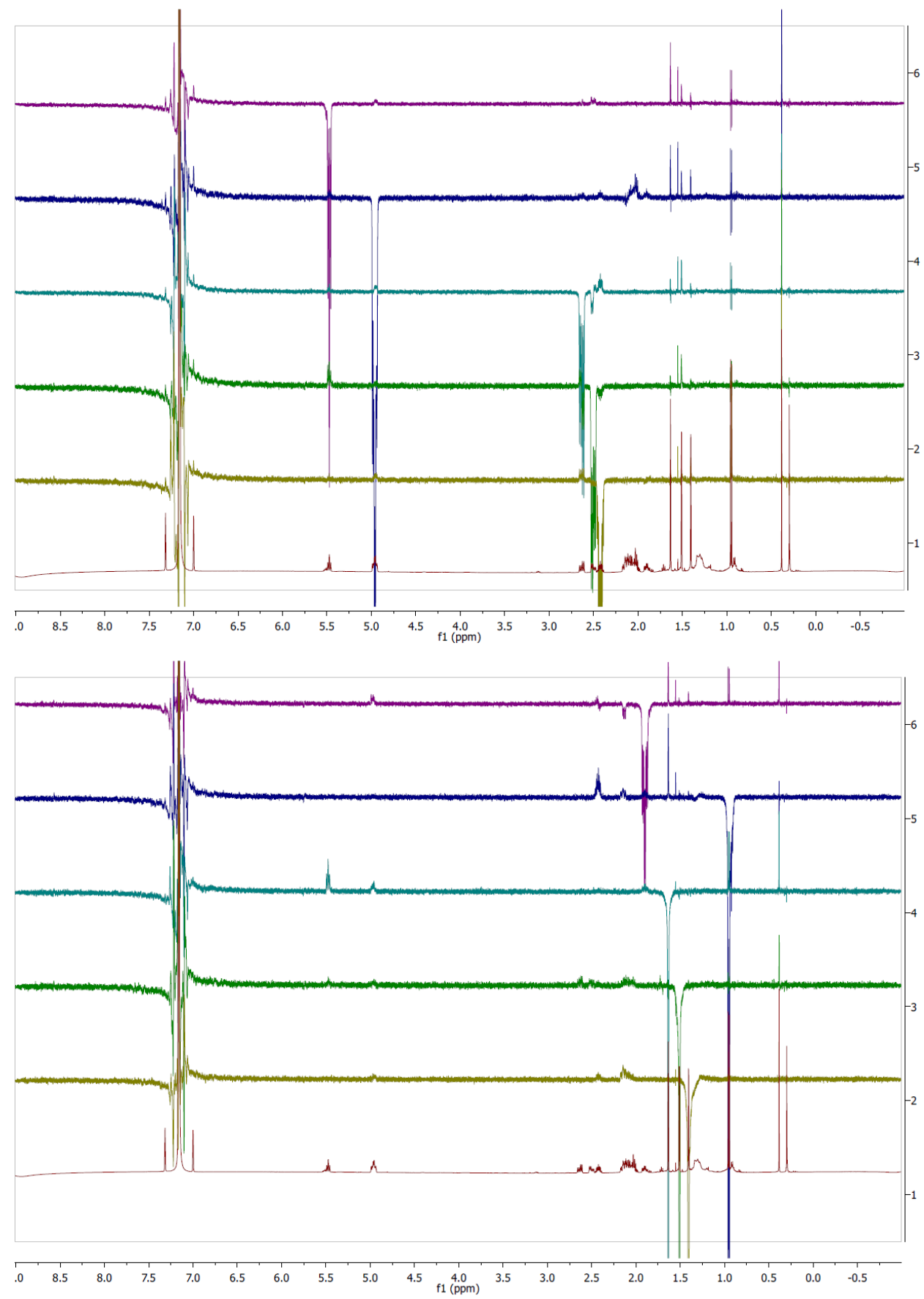

\subsection{Structure elucidation of 26 and 27}

Products 26 and 27 were isolated from the biotransformation of Bot2 with non-natural substrate $\mathbf{1 1}$ in $32.6 \%$ yield. Purification was done by pGC. The purified products (each $1 \mathrm{mg}$ ) were solved in $\mathrm{C}_{6} \mathrm{D}_{6}$ and analysed with NMR and HR-GCMS.

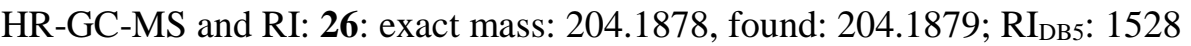

27: exact mass: 204.1878, found: 204: 1877; $\mathrm{RI}_{\mathrm{DB} 5}: 1520$ 


\section{Product 26:}

\begin{tabular}{|c|c|c|c|c|c|c|c|c|}
\hline $\mathrm{Nr}$ & $\delta^{13 C}$ & $\delta^{1 \mathrm{H}}$ & $\# \mathbf{H}$ & Multi & $\mathrm{J}(\mathrm{Hz})$ & COSY & HMBC & NOESY \\
\hline 1 & 131.9 & & & & & & $3,11 a, 12$ & \\
\hline 2 & 128.0 & 5.14 & 1 & $\mathrm{t}$ & 7.6 & 3,12 & $3,11 a, 12$ & $3,9,11 a$ \\
\hline 3 & 25.8 & $\begin{array}{l}2.56- \\
2.61\end{array}$ & 2 & $\mathrm{~m}$ & & $2,4,12,13$ & 2,4 & $2,4,12$ \\
\hline 4 & 124.8 & 5.42 & 1 & ddq & $8.7,5.0,1.5$ & 3,13 & 6,13 & 3,13 \\
\hline 5 & 140.3 & & & & & & $\begin{array}{c}3,7 b, 13 \\
14\end{array}$ & \\
\hline 6 & 41.1 & $\begin{array}{l}2.53- \\
2.57\end{array}$ & 1 & $\mathrm{~m}$ & & $7 a, 7 b, 14$ & $\begin{array}{c}4,7 a, 7 b \\
13\end{array}$ & $7 b, 9,14$ \\
\hline $\begin{array}{l}7 a \\
7 b\end{array}$ & 44.9 & $\begin{array}{l}1.86 \\
2.14\end{array}$ & $\begin{array}{l}1 \\
1\end{array}$ & $\begin{array}{l}d d \\
d d\end{array}$ & $\begin{array}{c}10.8,12.6 \\
12.7,3.9\end{array}$ & $\begin{array}{l}6,7 b \\
6,7 a\end{array}$ & 14,15 & $\begin{array}{c}7 b, 9,13,14,15 \\
6,7 a, 9,14\end{array}$ \\
\hline 8 & 137.1 & & & & & & $7 a, 7 b, 15$ & \\
\hline 9 & 126.6 & 4.86 & 1 & $d d p$ & $10.5,5.0,1.3$ & $\begin{array}{c}10 a, 10 b, 11 a \\
15\end{array}$ & $\begin{array}{l}7 a, 7 b \\
11 a, 15\end{array}$ & $6,7 b, 10 a$ \\
\hline $\begin{array}{l}10 a \\
10 b\end{array}$ & 25.3 & $\begin{array}{l}1.99 \\
2.17\end{array}$ & $\begin{array}{l}1 \\
1\end{array}$ & $\begin{array}{l}\mathrm{dq} \\
\mathrm{dtd}\end{array}$ & $\begin{array}{c}12.9,5.0,4.3 \\
12.9,10.9,4.9\end{array}$ & $\begin{array}{c}9,11 a, 11 b \\
9,11 b\end{array}$ & $11 a, 11 b$ & $\begin{array}{c}10 \mathrm{~b}, 15,9 \\
10 \mathrm{~b}, 15\end{array}$ \\
\hline $\begin{array}{l}11 a \\
11 b\end{array}$ & 39.5 & $\begin{array}{l}1.93 \\
2.08\end{array}$ & $\begin{array}{l}1 \\
1\end{array}$ & $\begin{array}{l}\mathrm{td} \\
\mathrm{dt}\end{array}$ & $\begin{array}{c}11.7,10.9,5.0 \\
1.7,4.3\end{array}$ & $\begin{array}{c}10 a, 11 b \\
10 a, 10 b, 11 a\end{array}$ & $2,10 a, 12$ & $\begin{array}{l}2,11 \mathrm{~b} \\
12,11 \mathrm{a}\end{array}$ \\
\hline 12 & 16.5 & 1.44 & 3 & $\mathrm{t}$ & 1,3 & 2,3 & $2,11 a$ & $3,11 b$ \\
\hline 13 & 17.8 & 1.58 & 3 & $\mathrm{t}$ & 1,6 & 4,6 & 4 & $4,7 a, 14$ \\
\hline 14 & 20.7 & 0.96 & 3 & $d$ & 7,0 & 6 & & $6,7 a, 7 b, 13$ \\
\hline 15 & 18.3 & 1.47 & 3 & dd & 1,$5 ; 0,8$ & $10 a, 9$ & $7 a, 7 b$ & $7 a, 10 a, 10 b$ \\
\hline
\end{tabular}


${ }^{1} \mathrm{H}-\mathrm{NMR}(\mathbf{2 6})-600 \mathrm{MHz}, \mathrm{C}_{6} \mathrm{D}_{6}$

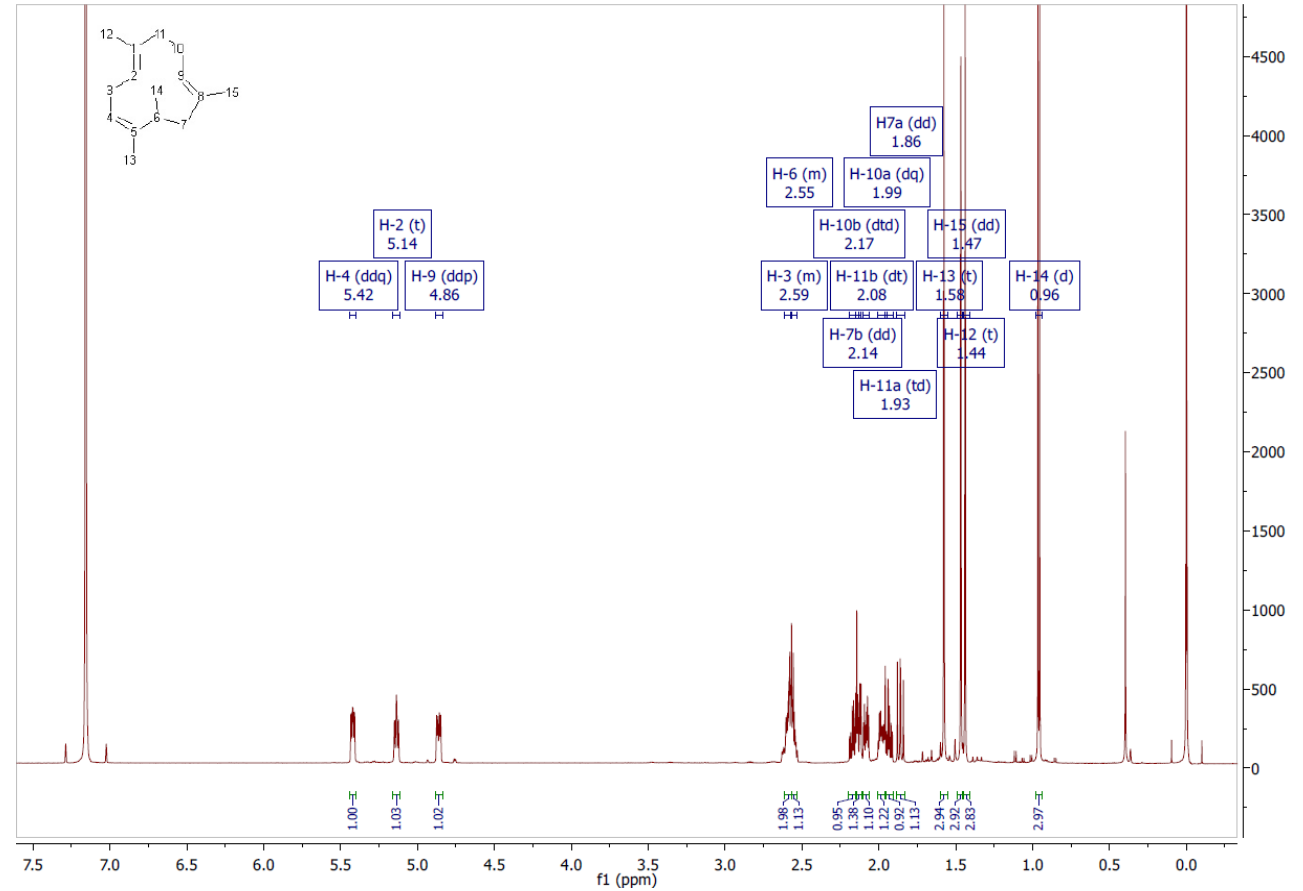

${ }^{13} \mathrm{C}-\mathrm{NMR}(\mathbf{2 6})$ - $151 \mathrm{MHz}, \mathrm{C}_{6} \mathrm{D}_{6}$

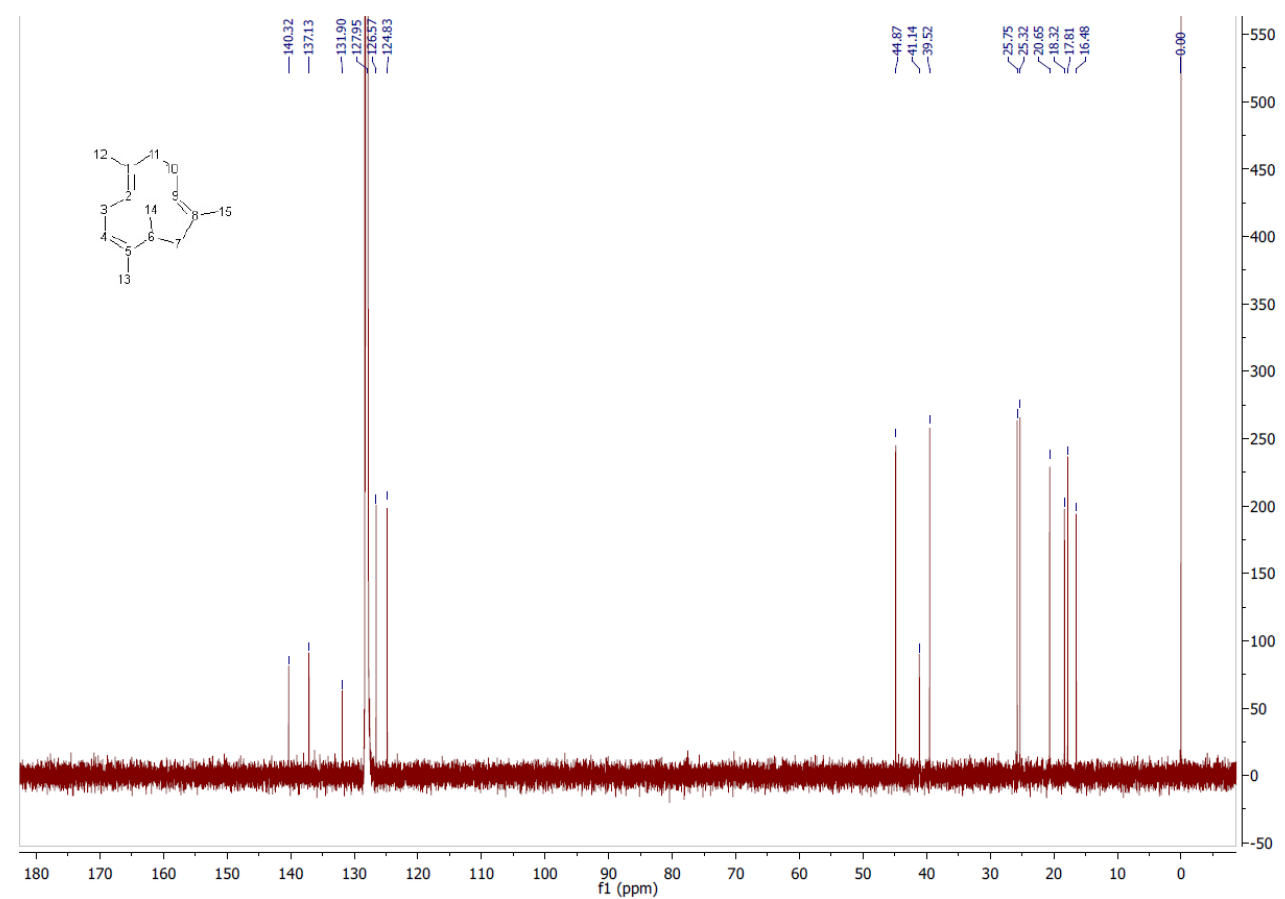


DEPT135 (26) - $151 \mathrm{MHz}, \mathrm{C}_{6} \mathrm{D}_{6}$

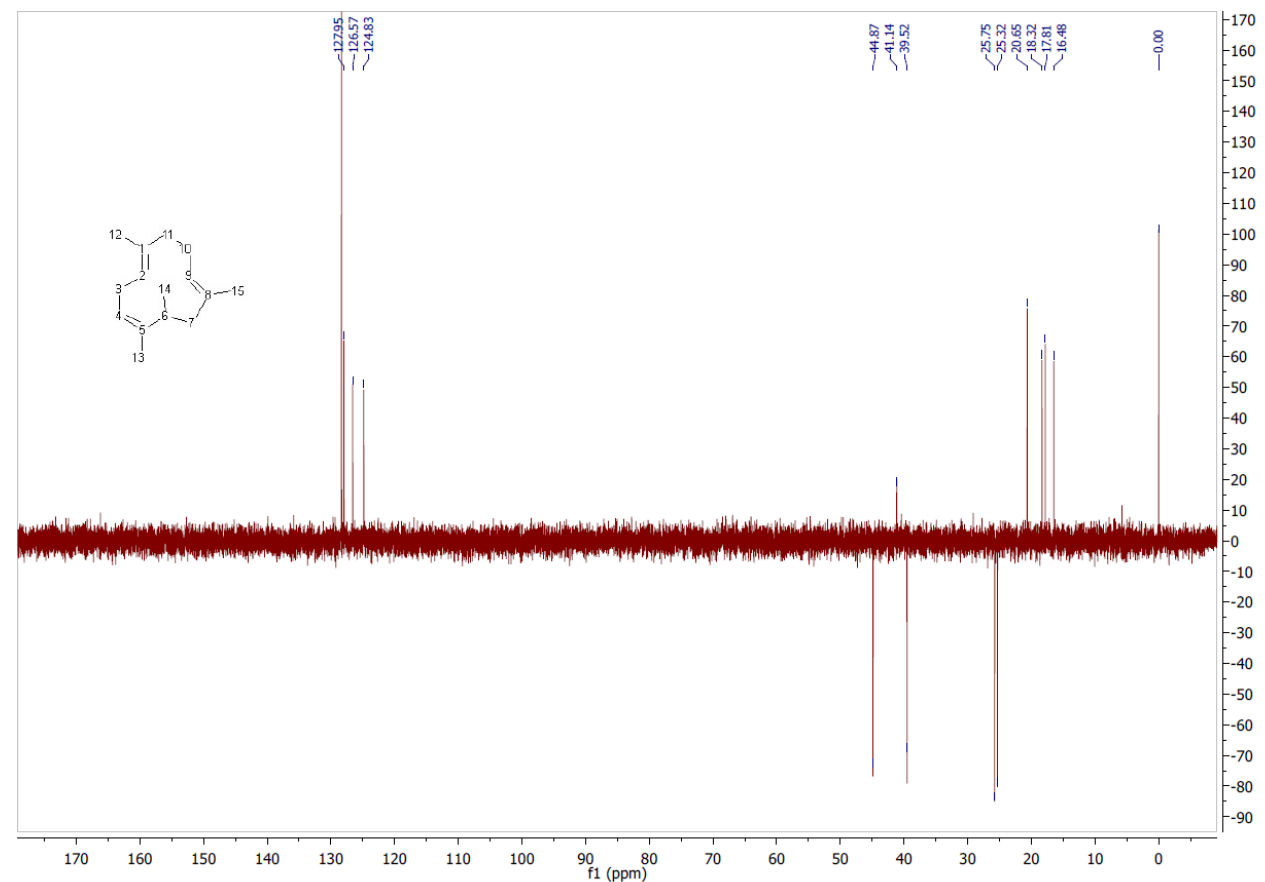

HSQC (26) - $600 \mathrm{MHz}, \mathrm{C}_{6} \mathrm{D}_{6}$

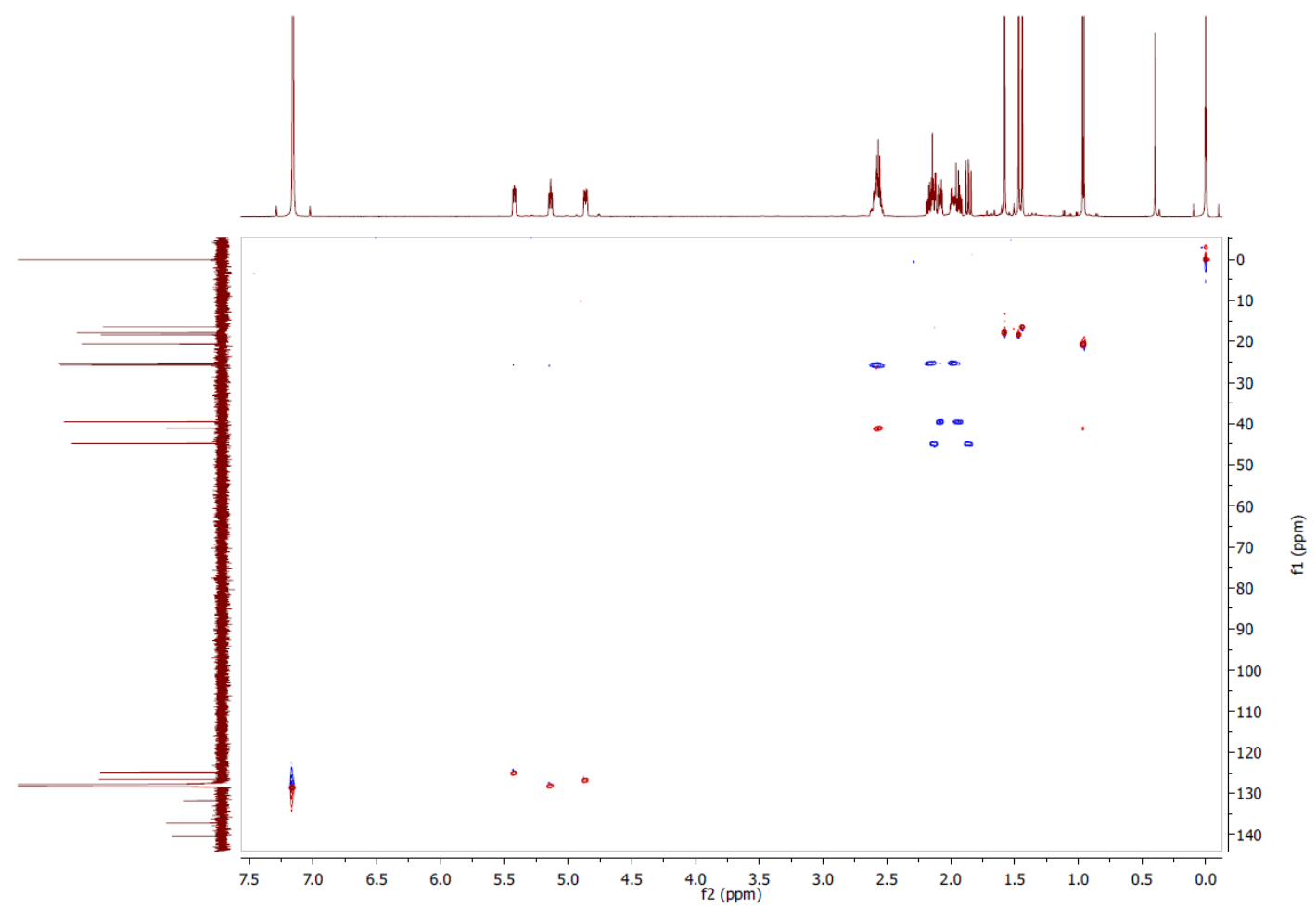


COSY (26) - $600 \mathrm{MHz}, \mathrm{C}_{6} \mathrm{D}_{6}$

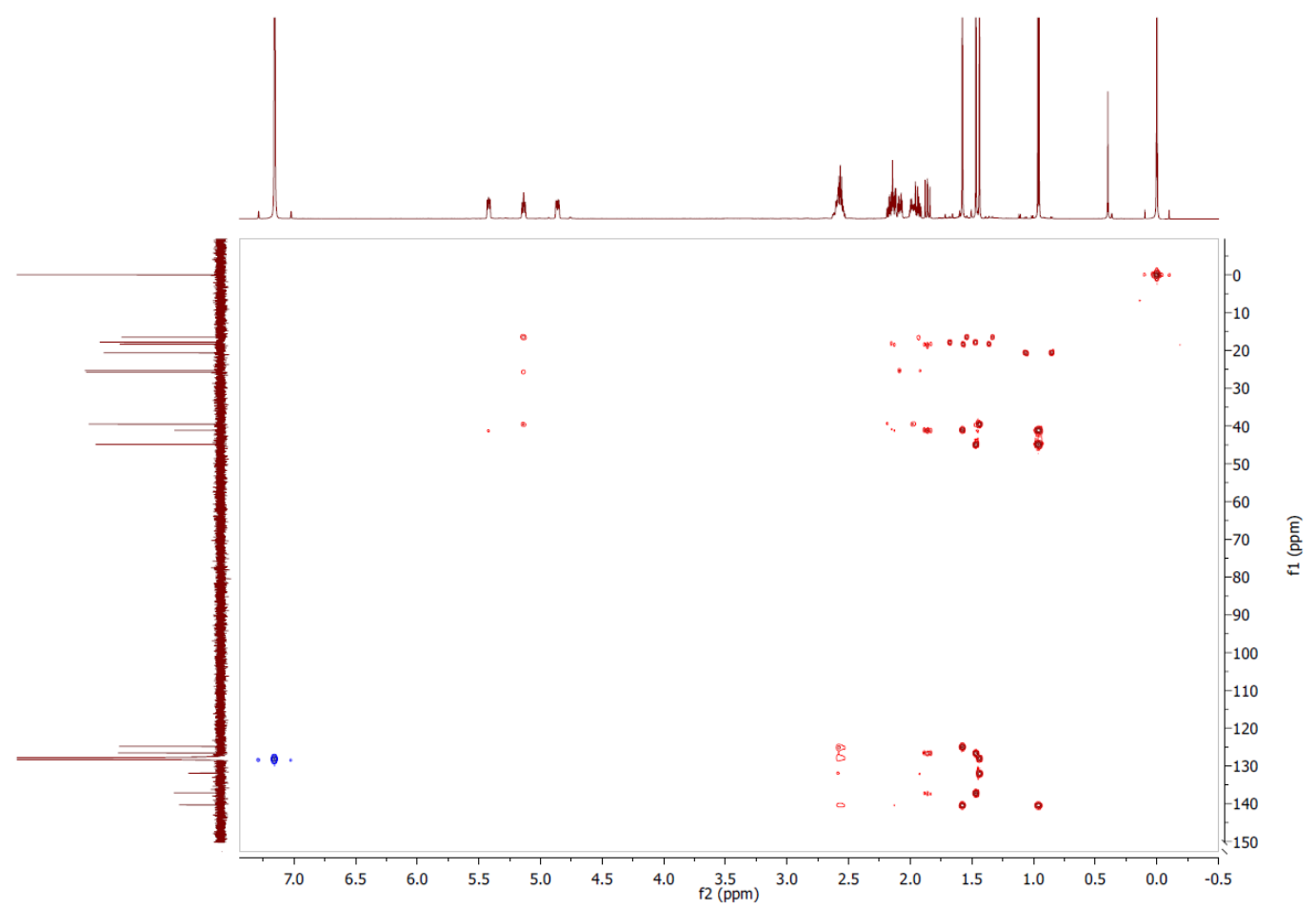

HMBC (26) - $600 \mathrm{MHz}, \mathrm{C}_{6} \mathrm{D}_{6}$

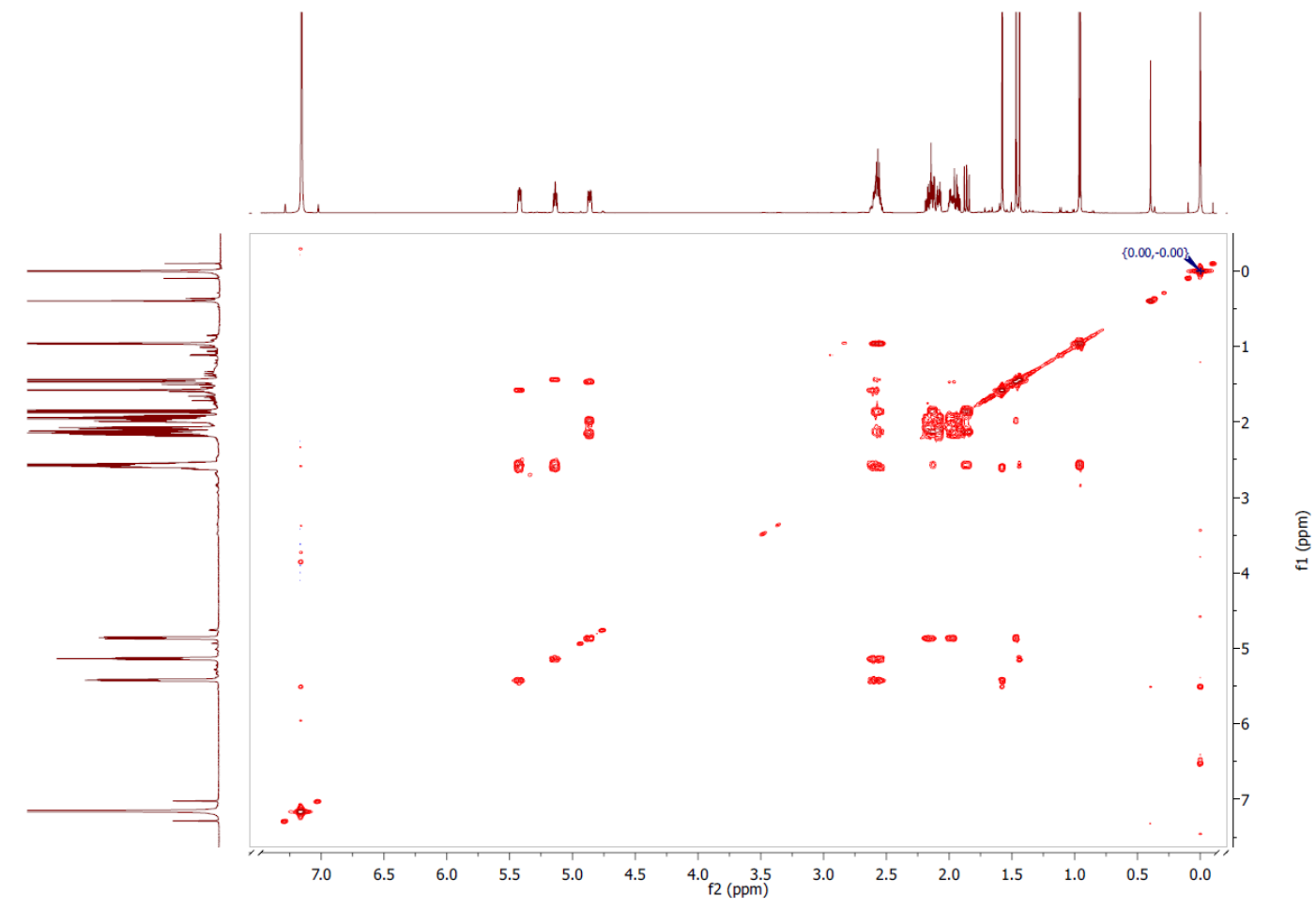


NOESY (26) - $600 \mathrm{MHz}, \mathrm{C}_{6} \mathrm{D}_{6}$

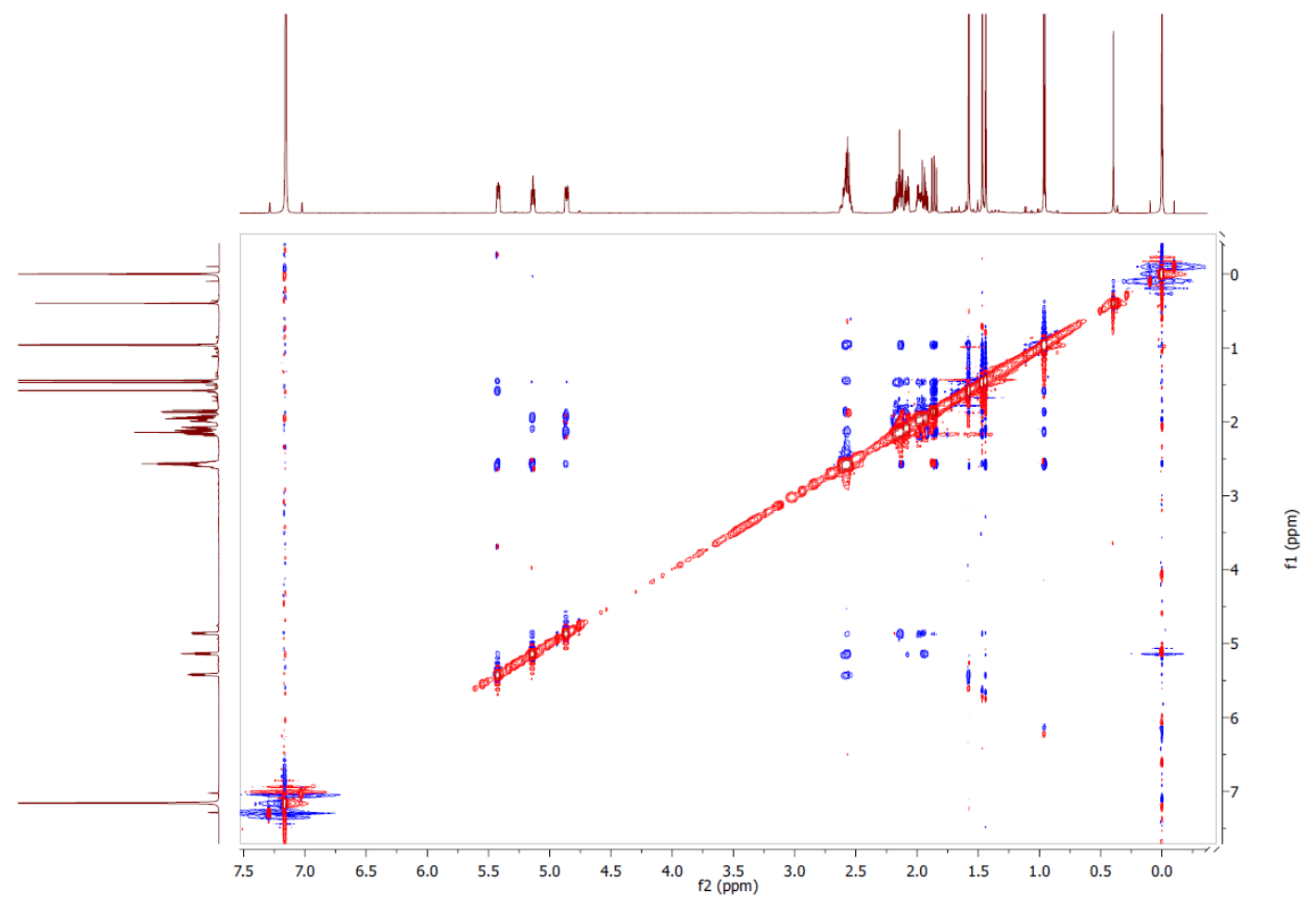

GC-IR (26)

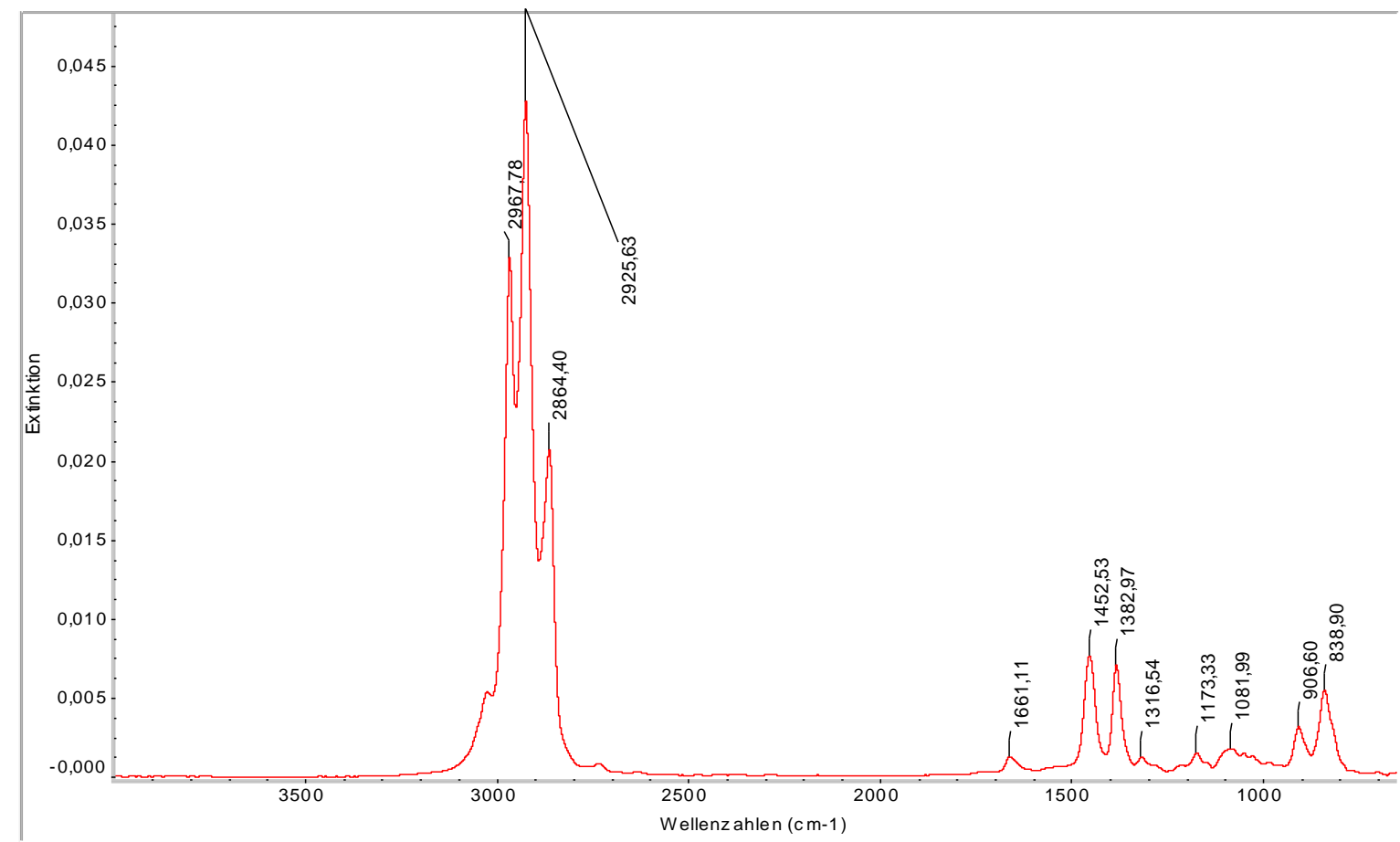




\section{Product 27:}

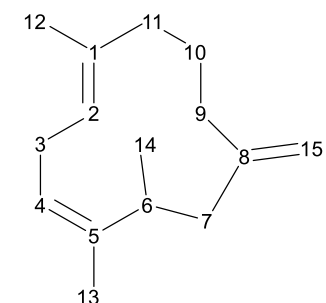

\begin{tabular}{|c|c|c|c|c|c|c|c|c|}
\hline $\mathrm{Nr}$ & $\delta^{13 c}$ & $\delta^{1 \mathrm{H}}$ & $\# \mathbf{H}$ & Multi & $\mathrm{J}(\mathrm{Hz})$ & $\cos Y$ & HMBC & NOESY \\
\hline 1 & 132.6 & & & & & & $\begin{array}{c}10,11 a, 11 b, \\
12\end{array}$ & \\
\hline 2 & 127.0 & 5.33 & 1 & $\mathrm{dh}$ & $\begin{array}{c}9.3,4.4,1.3 \\
0.7\end{array}$ & $3 a, 3 b, 12$ & 11a, 11b, 12 & $3 b, 6,11 a, 11 b$ \\
\hline $3 a$ & 26.3 & $\begin{array}{r}2.52- \\
2.46\end{array}$ & 1 & $\mathrm{~m}$ & & $2,3 b, 4,12$ & & $3 b, 4,12$ \\
\hline $3 b$ & & 2.70 & 1 & dddt & $\begin{array}{c}18.2,9.3 \\
4.3,2.1\end{array}$ & $2,3 a, 4,13$ & & $2,3 a, 12$ \\
\hline 4 & 123.4 & 5.40 & 1 & ddq & $8.7,4.3,1.5$ & $3 a, 3 b, 13$ & 6,13 & $3 a, 13$ \\
\hline 5 & 141.4 & & & & & & $6,7 a, 7 b, 13$ & \\
\hline 6 & 34.1 & 2.84 & 1 & $\mathrm{pd}$ & $7.8,7.0,5.2$ & $7 a, 7 b, 14$ & $7 a, 7 b, 13,14$ & $2,7 a, 14$ \\
\hline $7 a$ & 43.1 & 1.75 & 1 & dd & $13.1,5.2$ & $6,7 b$ & $6,14,15 a, 15 b$ & $6,7 b, 14,15 a$ \\
\hline $7 b$ & & 2.17 & 1 & dd & $13.1,7.8$ & $6,7 a$ & & $7 a, 14,15 a$ \\
\hline 8 & 148.4 & & & & & & $7 a, 7 b, 10$ & \\
\hline $\begin{array}{l}9 a \\
9 b\end{array}$ & 32.4 & $\begin{array}{l}1.78 \\
2.03\end{array}$ & $\begin{array}{l}1 \\
1\end{array}$ & $\begin{array}{c}d t \\
d d t\end{array}$ & $\begin{array}{c}16.2,5.7 \\
16.2,5.5,1.8\end{array}$ & $\begin{array}{l}9 b, 10 \\
9 a, 10\end{array}$ & $\begin{array}{c}7 a, 7 b, 10 \\
11 a, 11 b, 15 a \\
15 b\end{array}$ & $\begin{array}{c}9 b \\
9 a, 15 b\end{array}$ \\
\hline 10 & 23.6 & $\begin{array}{l}1.63- \\
1.57\end{array}$ & 2 & $\mathrm{~m}$ & & $\begin{array}{c}9 a, 9 b, 11 a \\
11 b\end{array}$ & $11 a, 11 b$ & $15 b$ \\
\hline $\begin{array}{l}11 a \\
11 b\end{array}$ & 38.7 & $\begin{array}{l}1.85 \\
2.09\end{array}$ & $\begin{array}{l}1 \\
1\end{array}$ & $\begin{array}{l}d t \\
d t\end{array}$ & $\begin{array}{l}13.1,7.4 \\
13.1,5.4\end{array}$ & $\begin{array}{l}10,11 \mathrm{~b} \\
10,11 \mathrm{a}\end{array}$ & $9 a, 9 b, 10,12$ & $\begin{array}{l}2,11 b \\
2,11 b\end{array}$ \\
\hline 12 & 17.1 & 1.50 & 3 & $\mathrm{t}$ & 1.3 & $2,3 a$ & $11 a$ & $3 a, 3 b$ \\
\hline 13 & 17.8 & 1.60 & 3 & $\mathrm{t}$ & 1.7 & $3 a, 4$ & 6 & 4,14 \\
\hline 14 & 19.7 & 0.95 & 3 & $d$ & 7.0 & 6 & $6,7 a, 7 b$ & $6,7 a, 7 b, 13$ \\
\hline $15 a$ & 109.7 & $\begin{array}{r}4.76- \\
4.74\end{array}$ & 1 & $\mathrm{~m}$ & & $9 a, 9 b, 15 b$ & $7 a, 7 b$ & $7 a, 7 b, 15 b$ \\
\hline $15 b$ & & 4.76 & 1 & $q$ & 1.8 & $9 a, 9 b, 15 a$ & & $9 b, 10,15 a$ \\
\hline
\end{tabular}


${ }^{1} \mathrm{H}-\mathrm{NMR}$ (27) - $600 \mathrm{MHz}, \mathrm{C}_{6} \mathrm{D}_{6}$

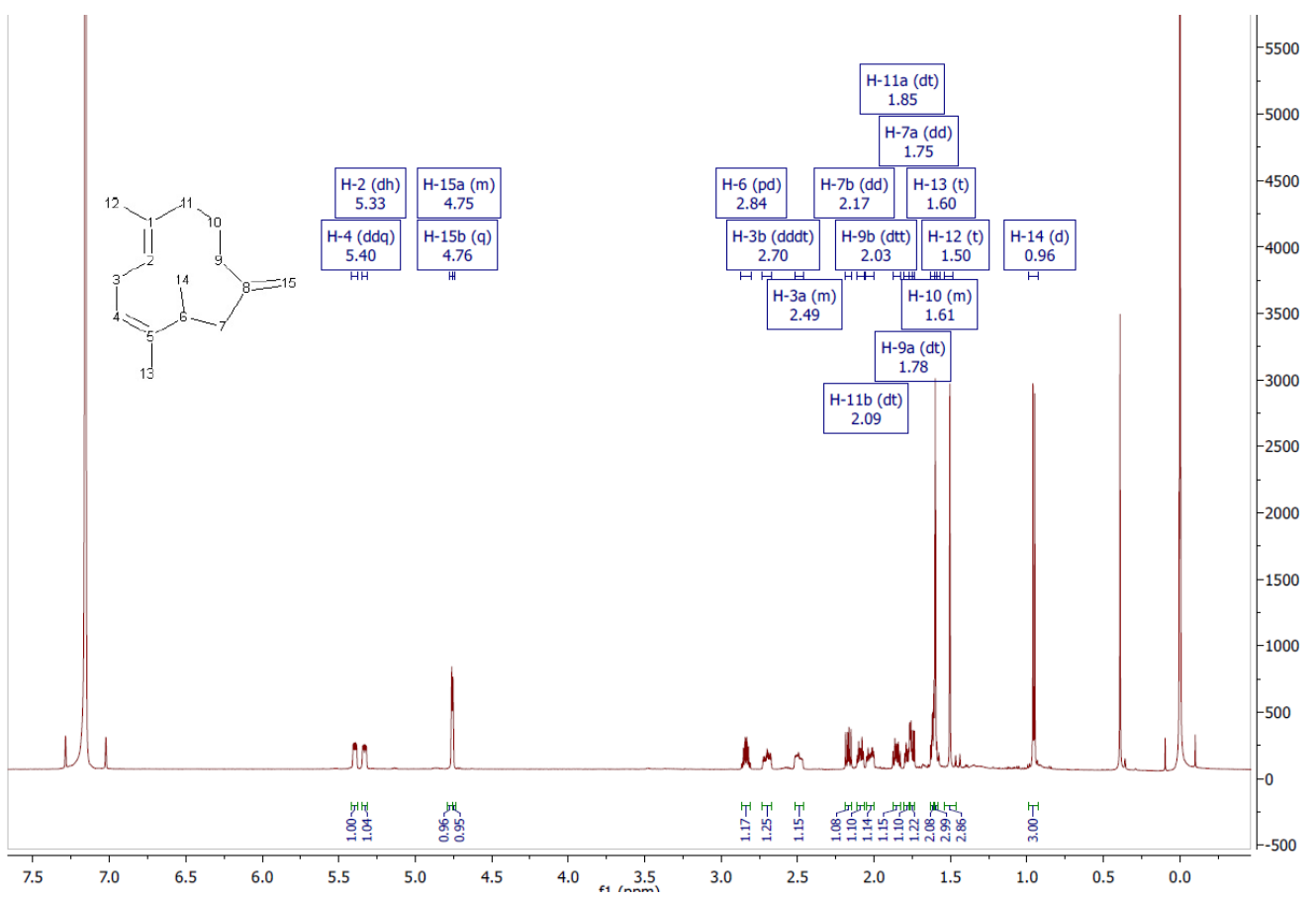

${ }^{13} \mathrm{C}-\mathrm{NMR}(27)-151 \mathrm{MHz}, \mathrm{C}_{6} \mathrm{D}_{6}$

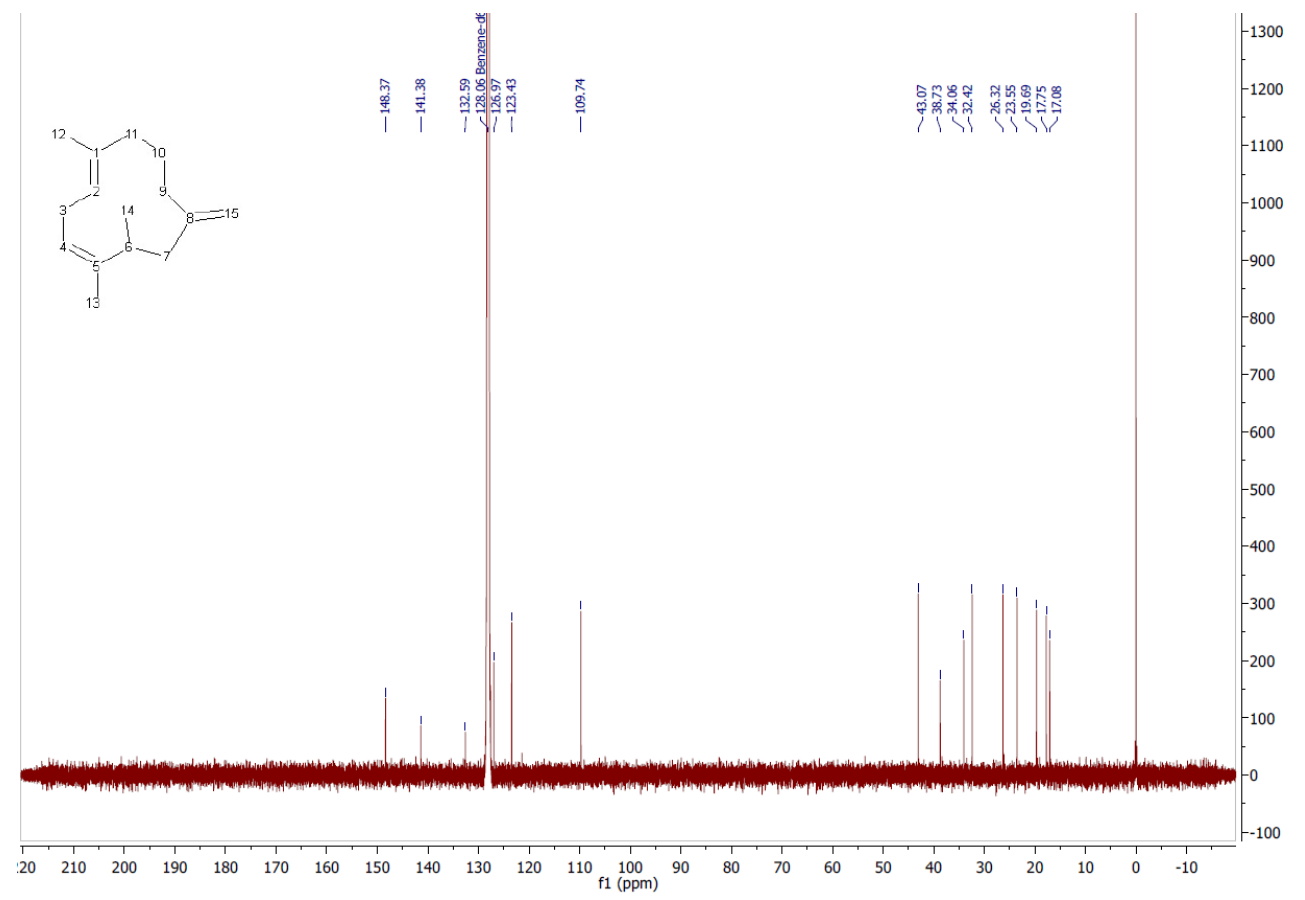


DEPT135 (27) - $151 \mathrm{MHz}, \mathrm{C}_{6} \mathrm{D}_{6}$

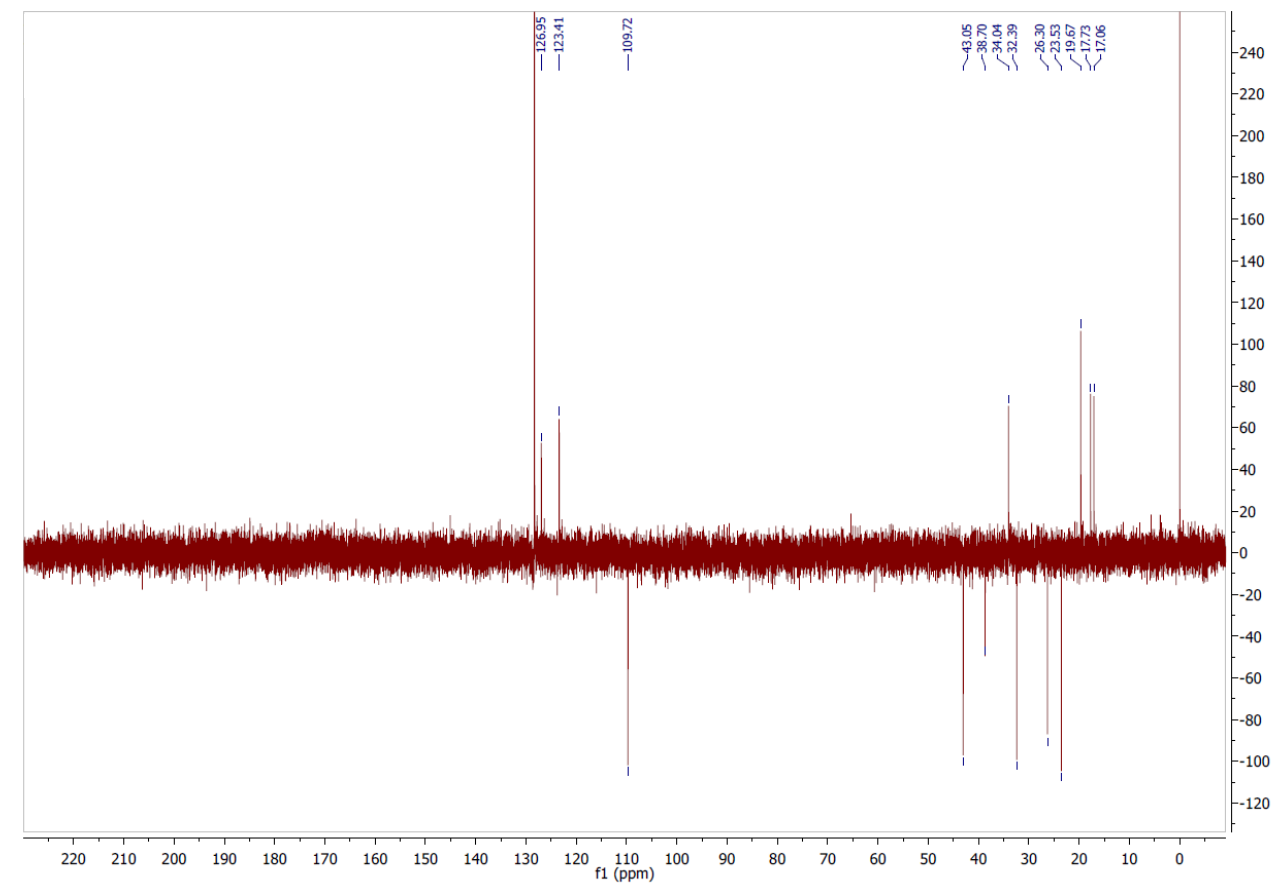

HSQC (27) - $600 \mathrm{MHz}, \mathrm{C}_{6} \mathrm{D}_{6}$

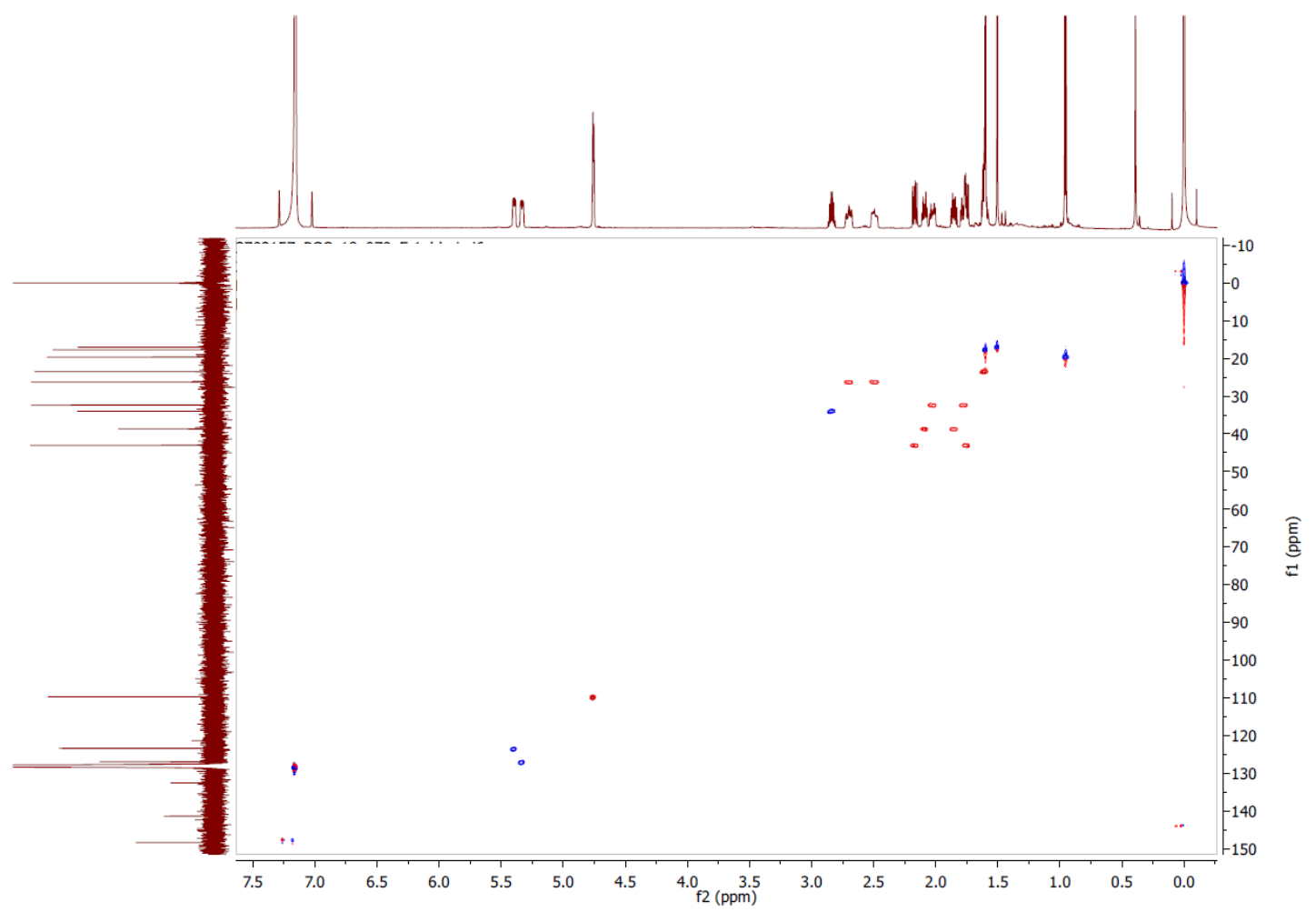


$\operatorname{COSY}(27)-600 \mathrm{MHz}, \mathrm{C}_{6} \mathrm{D}_{6}$

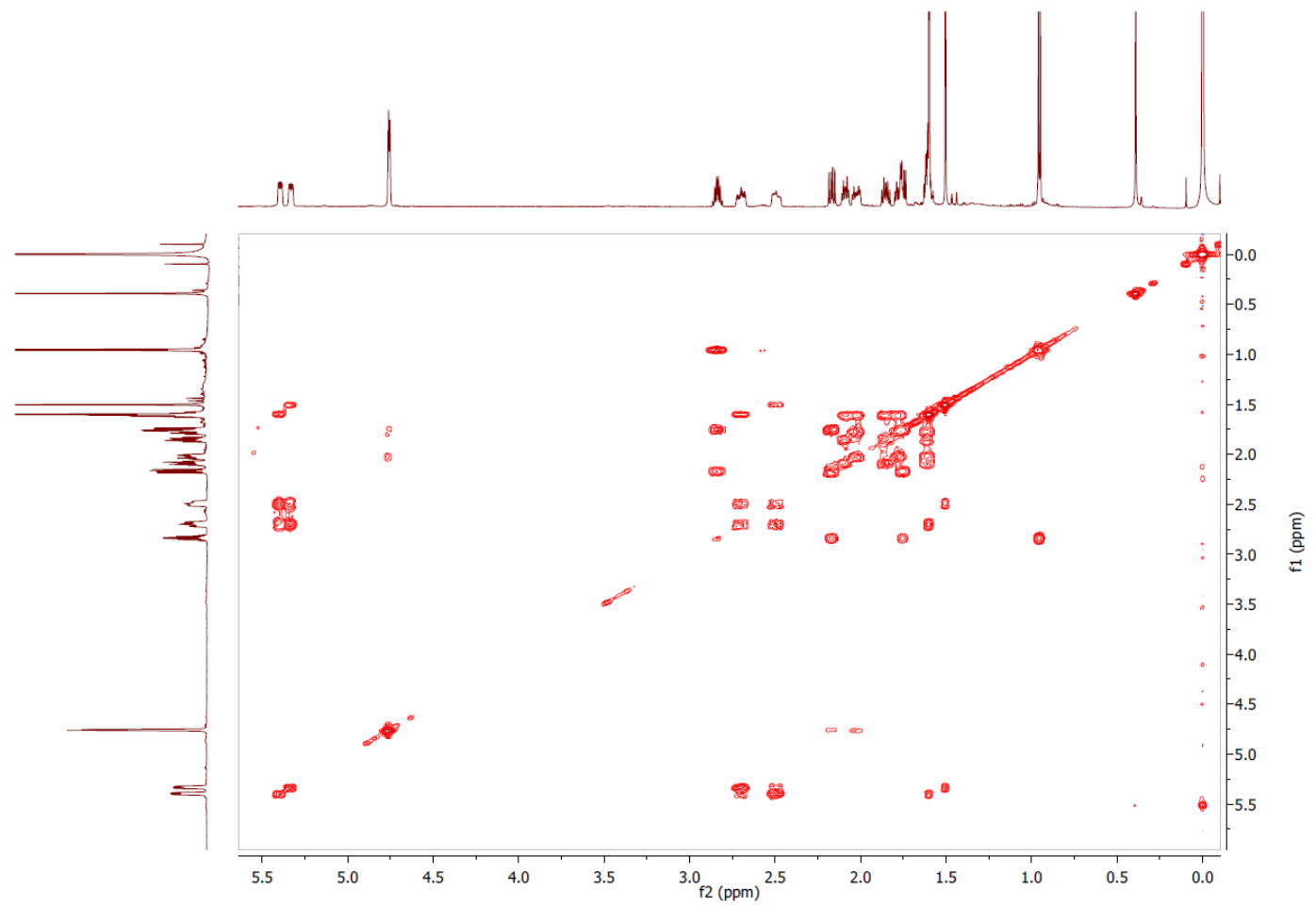

HMBC (27) - $600 \mathrm{MHz}, \mathrm{C}_{6} \mathrm{D}_{6}$

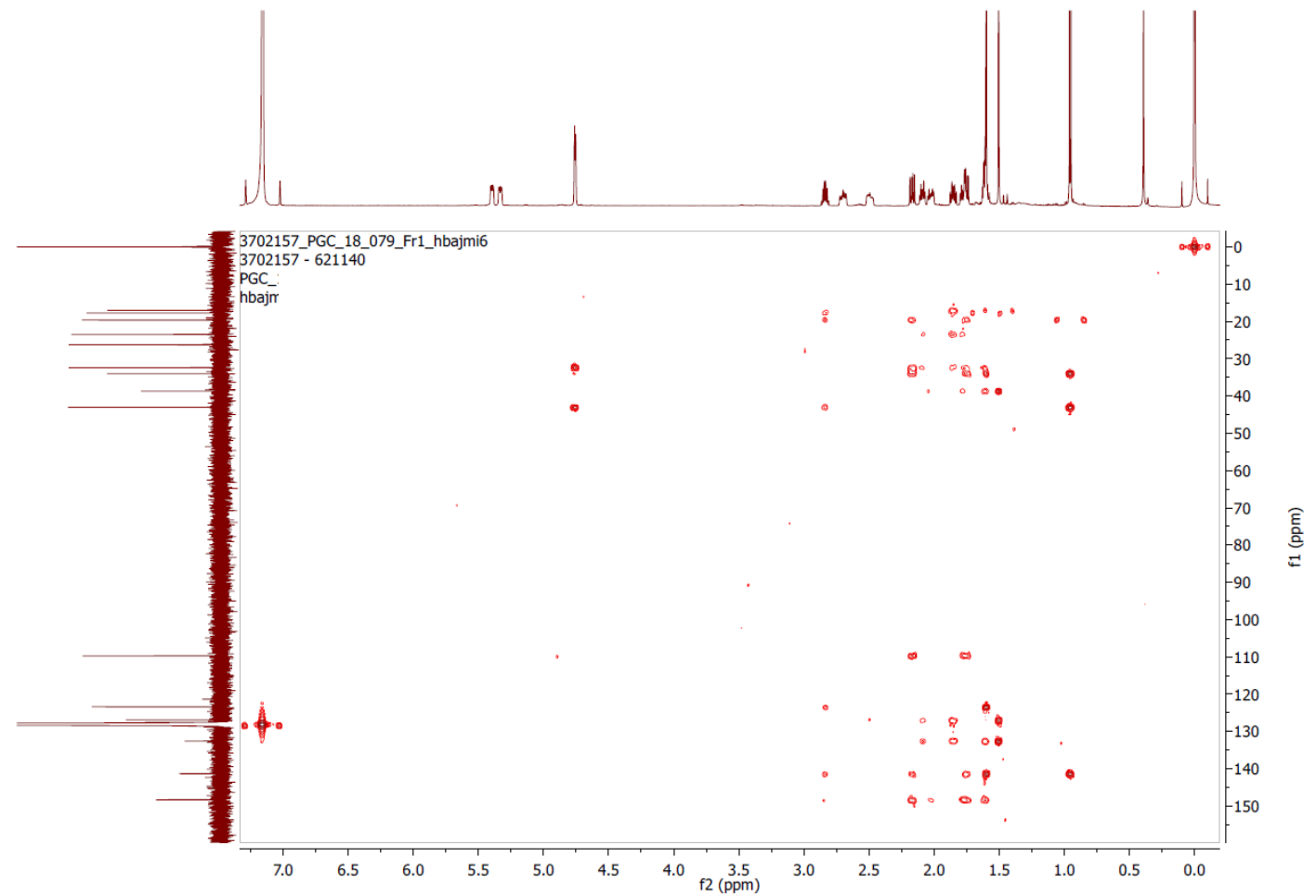


NOESY (27) - $600 \mathrm{MHz}, \mathrm{C}_{6} \mathrm{D}_{6}$

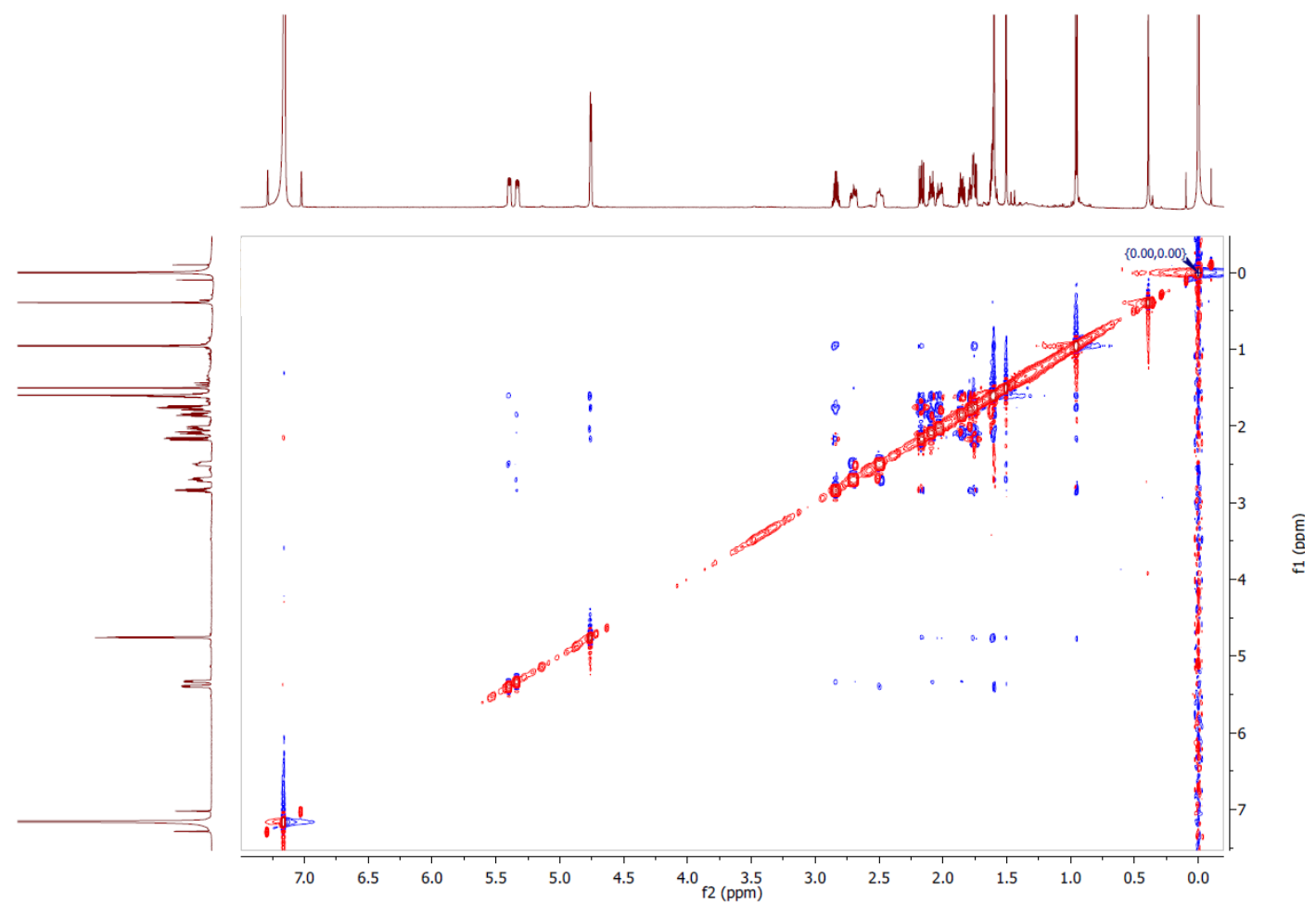

1d-NOE (27) - $600 \mathrm{MHz}, \mathrm{C}_{6} \mathrm{D}_{6}$

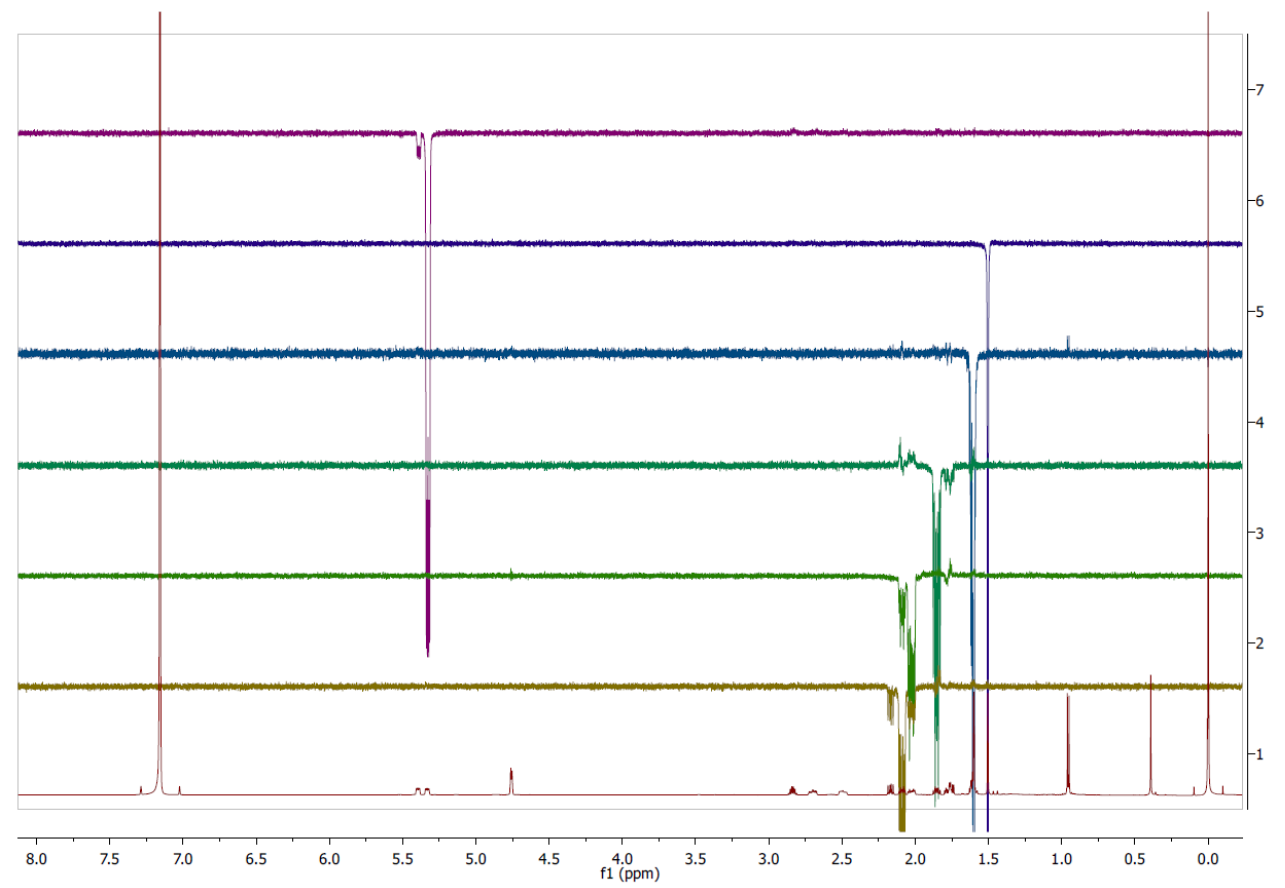




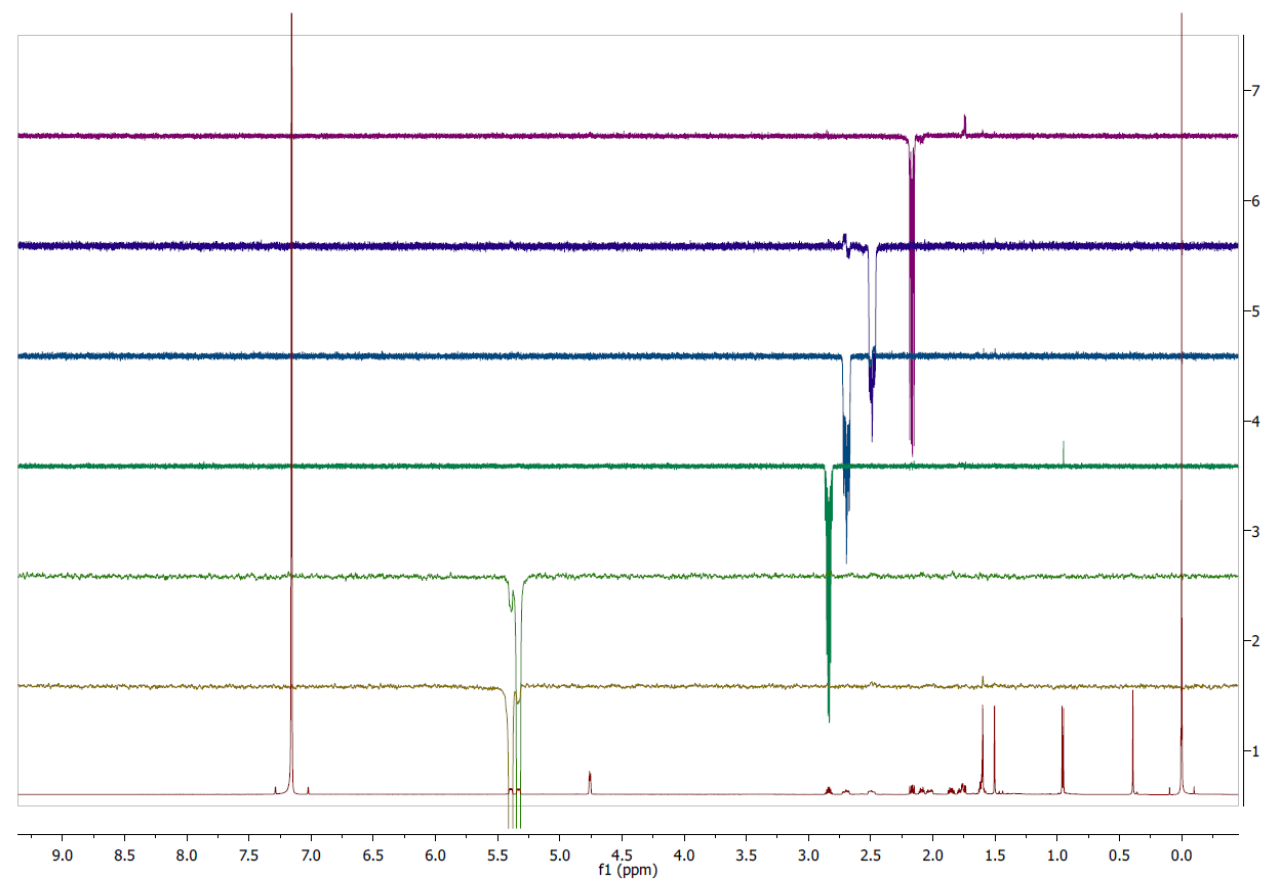

GC-IR (27)

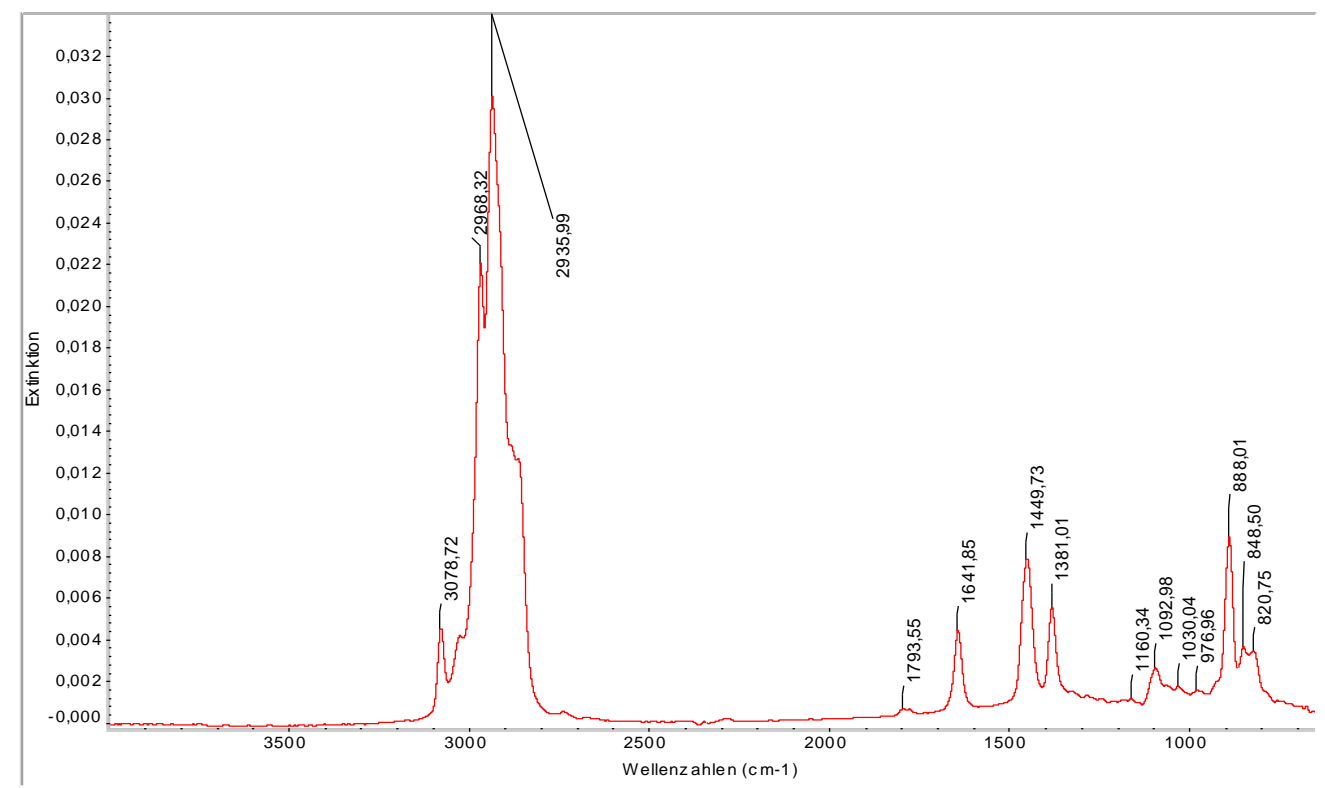

\subsection{Structure elucidation of 28}

Product 28 was isolated from the biotransformation of Bot 2 with non-natural substrate $\mathbf{1 2}$ in $30.6 \%$ yield. Purification was done by pHPLC. The purified product was solved in $\mathrm{C}_{6} \mathrm{D}_{6}$ for NMR at $325 \mathrm{~K}$ and in toluene- $\mathrm{d}_{8}$ for NMR at $255 \mathrm{~K}$, respectively. NMR measurement at 255 $\mathrm{K}$ revealed three sets of signals, which can be assigned to the three conformers DD, UD and UU.

HR-GC-MS: exact mass: 204.1878, found: 204.1876.

RIDB5: 1497 
Product 28 at 298K, solved in $\mathrm{C}_{6} \mathrm{D}_{6}$ :

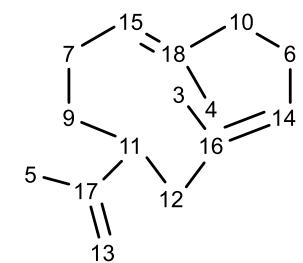

\begin{tabular}{|c|c|c|c|c|c|c|c|c|}
\hline $\mathrm{Nr}$ & $\delta^{13 c}$ & $\delta^{1 \mathrm{H}}$ & \#H & Multi & $\mathrm{J}(\mathrm{Hz})$ & cosy & HMBC & 1d-noe \\
\hline 3 & 16.7 & 1.34 & 3 & $\mathrm{~s}$ & & $12 b, 14,6 a$ & $12 a$ & $4,10 a, 12 b$ \\
\hline 4 & 16.8 & 1.44 & 3 & $\mathrm{~s}$ & & $7 b, 15$ & $10 a, 7 a$ & $3,10 b$ \\
\hline 5 & 20.0 & 1.64 & 3 & s & & $13 a, 13 b$ & $13 a, 13 b, 11$ & 13 \\
\hline $\begin{array}{l}6 a \\
6 b\end{array}$ & 26.8 & $\begin{array}{l}1.99 \\
2.15\end{array}$ & 2 & $\begin{array}{l}\mathrm{m} \\
\mathrm{dd}\end{array}$ & $4.7,12.2$ & $\begin{array}{c}6 b, 10 a, 10 b, 14 \\
6 a, 10 a, 14\end{array}$ & $10 a$ & \\
\hline $\begin{array}{l}7 a \\
7 b\end{array}$ & 29.0 & $\begin{array}{l}1.92 \\
7.22\end{array}$ & 2 & $\begin{array}{l}\mathrm{m} \\
\mathrm{dd}\end{array}$ & $6.7,14.4$ & $\begin{array}{c}7 b \\
4,7 a, 9 b\end{array}$ & $9 b, 11$ & \\
\hline $\begin{array}{l}9 a \\
9 b\end{array}$ & 37.8 & $\begin{array}{l}1.28 \\
1.86\end{array}$ & 2 & $\begin{array}{l}\mathrm{m} \\
\mathrm{m}\end{array}$ & & $\begin{array}{l}9 b, 11 \\
9 a, 7 b\end{array}$ & $11,12 a$ & $\begin{array}{c}9 b, 10 a, 15 \\
9 a\end{array}$ \\
\hline $\begin{array}{l}10 a \\
10 b\end{array}$ & 40.2 & $\begin{array}{l}1.92 \\
2.12\end{array}$ & 2 & $\begin{array}{l}\mathrm{m} \\
\mathrm{d}\end{array}$ & 9.5 & $\begin{array}{c}6 a, 6 b, 10 b \\
6 a, 10 a\end{array}$ & 4,6 & \\
\hline 11 & 47.4 & 2.09 & 1 & $\mathrm{~m}$ & & $9 a, 9 b$ & $13 a, 13 b, 5$ & 3 \\
\hline $\begin{array}{l}12 a \\
12 b\end{array}$ & 48.5 & $\begin{array}{l}2.09 \\
2.31\end{array}$ & 2 & $\begin{array}{l}\mathrm{m} \\
\mathrm{d}\end{array}$ & 10.2 & $\begin{array}{c}12 \mathrm{~b} \\
12 \mathrm{a}, 3\end{array}$ & $3,9 b$ & \\
\hline $\begin{array}{l}13 a \\
13 b\end{array}$ & 107.7 & $\begin{array}{l}4.66 \\
4.76\end{array}$ & 2 & s & & $\begin{array}{c}5,13 b \\
5\end{array}$ & 11,5 & 5 \\
\hline 14 & 127.6 & 4.76 & 1 & $\mathrm{~m}$ & & $6 a, 6 b, 3$ & $\begin{array}{l}3,6 b, 10 b, \\
12 a\end{array}$ & \\
\hline 18 & 127.8 & & & & & & $4,7 a, 10 a$ & \\
\hline 15 & 133.3 & 4.44 & 1 & $d$ & 10.8 & $4,7 a, 7 b, 10 a$ & $4,9 b, 10 a$ & \\
\hline 16 & 136.8 & & & & & & $3,6,12 a$ & \\
\hline 17 & 153.8 & & & & & & $5,9 b, 12 a$ & \\
\hline
\end{tabular}


${ }^{1} \mathrm{H}-\mathrm{NMR}\left(28-600 \mathrm{MHz}, \mathrm{C}_{6} \mathrm{D}_{6}\right)$

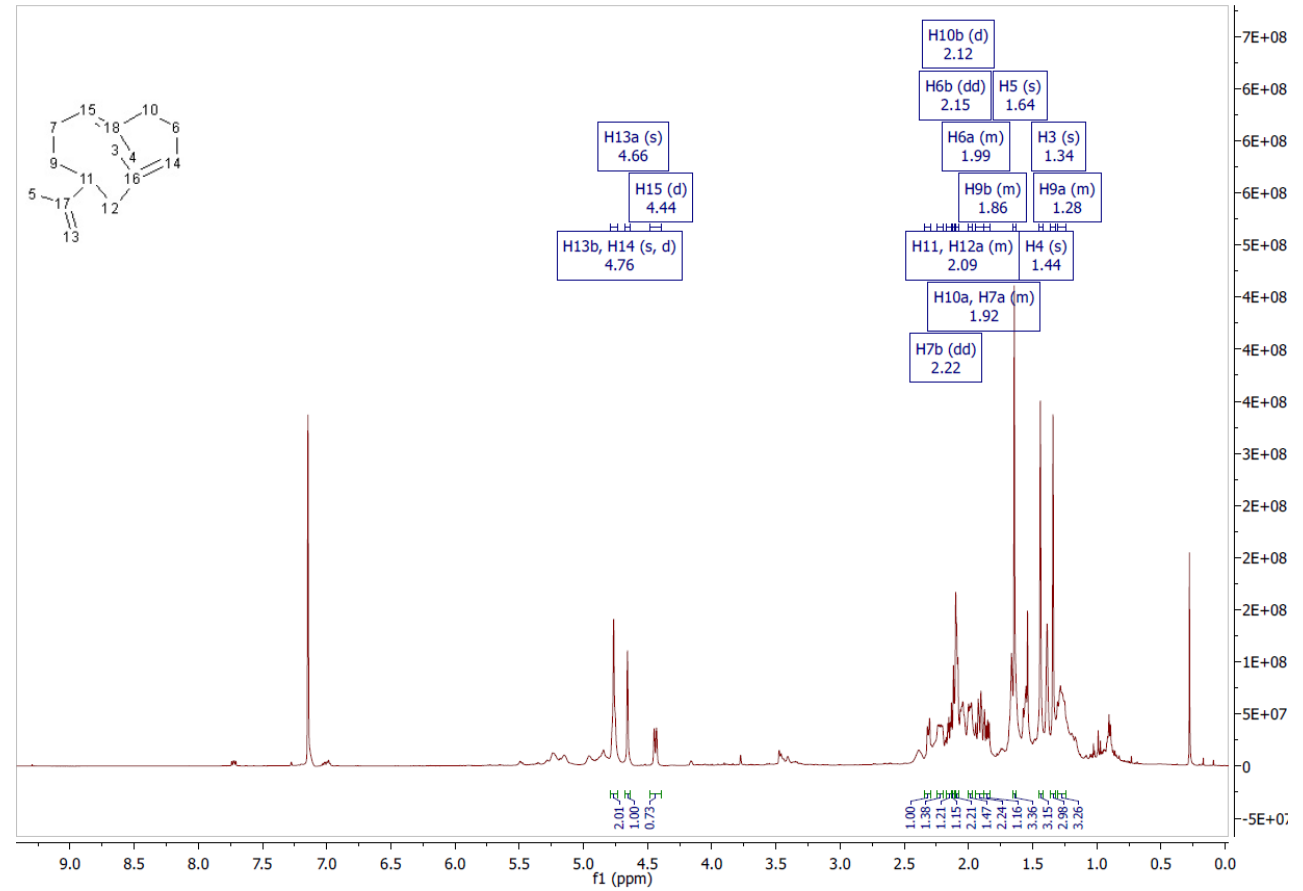

${ }^{13} \mathrm{C}-\mathrm{NMR}\left(28\right.$ - $\left.101 \mathrm{MHz}, \mathrm{C}_{6} \mathrm{D}_{6}\right)$

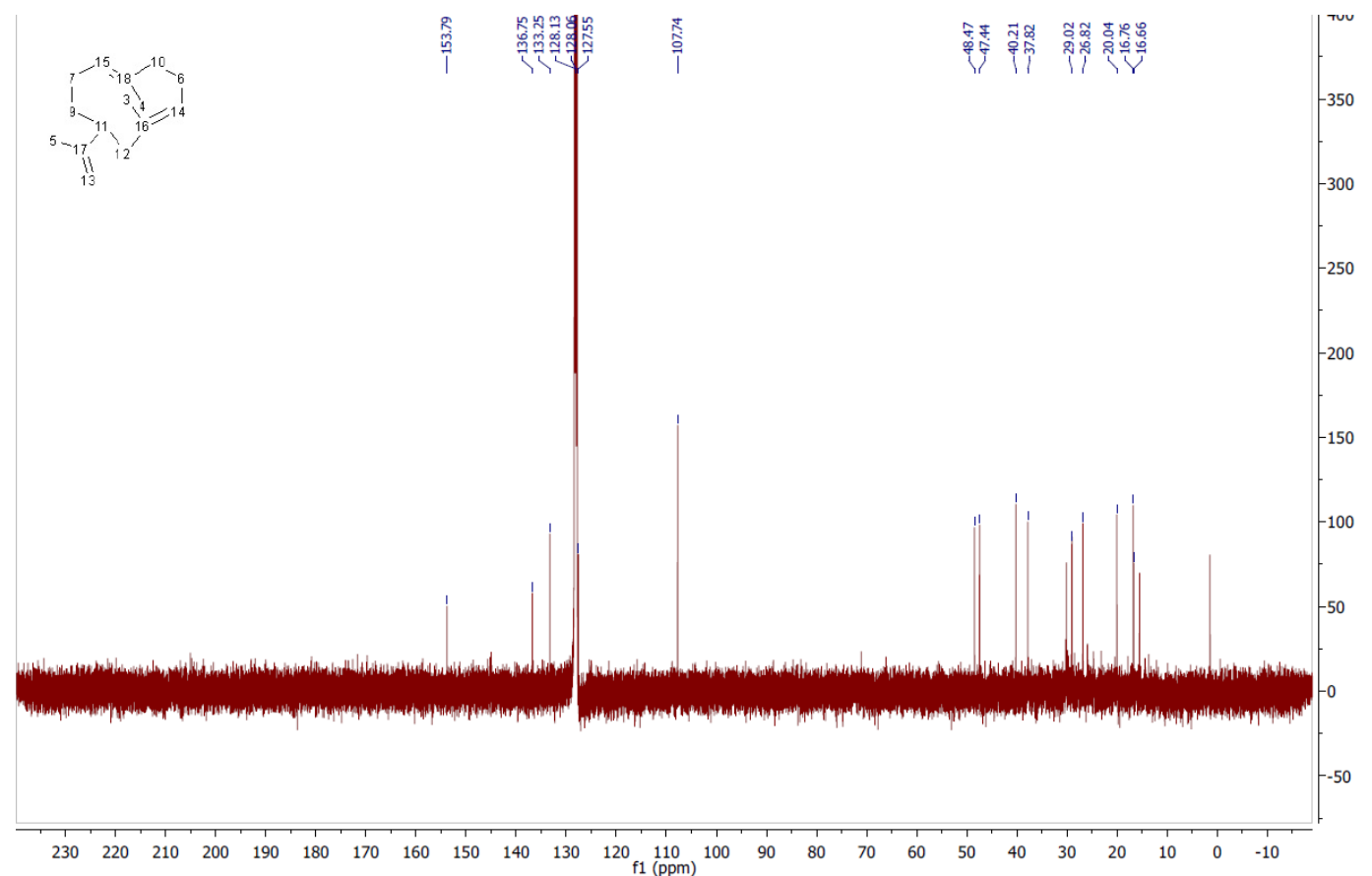


DEPT135 (28 - $\left.101 \mathrm{MHz}, \mathrm{C}_{6} \mathrm{D}_{6}\right)$

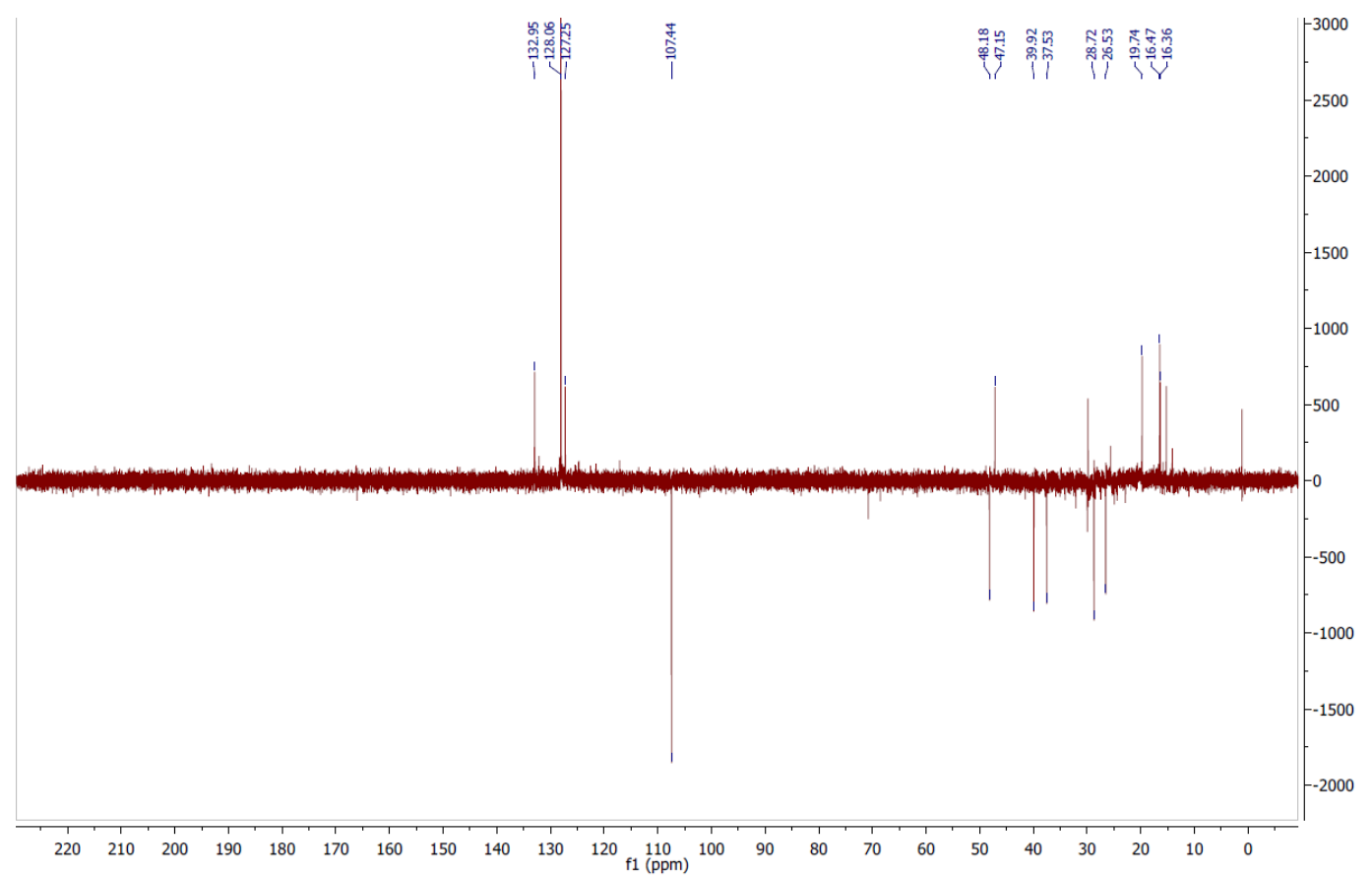

HSQC (28 - $\left.600 \mathrm{MHz}, \mathrm{C}_{6} \mathrm{D}_{6}\right)$

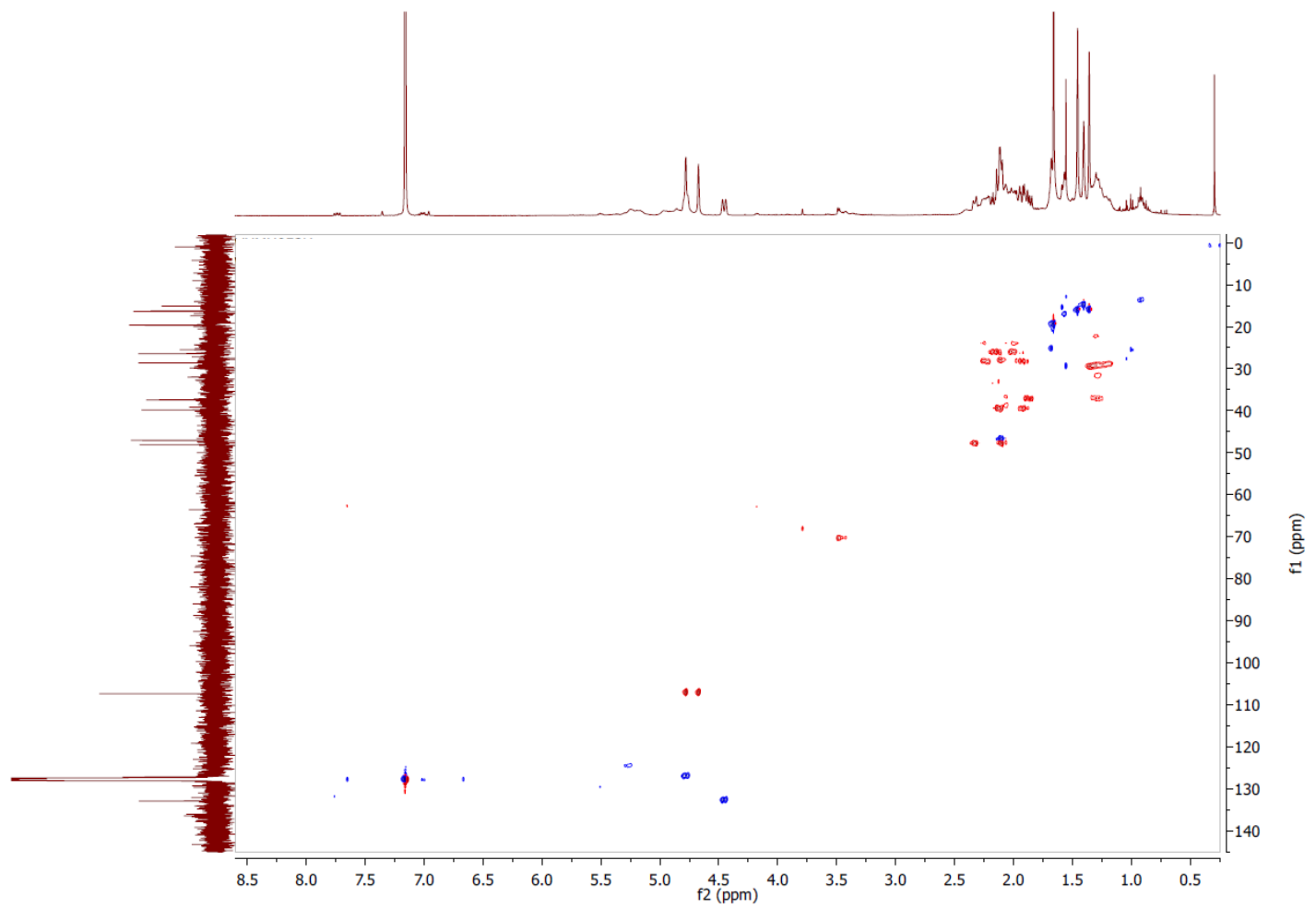


$\operatorname{COSY}\left(28-600 \mathrm{MHz}, \mathrm{C}_{6} \mathrm{D}_{6}\right)$

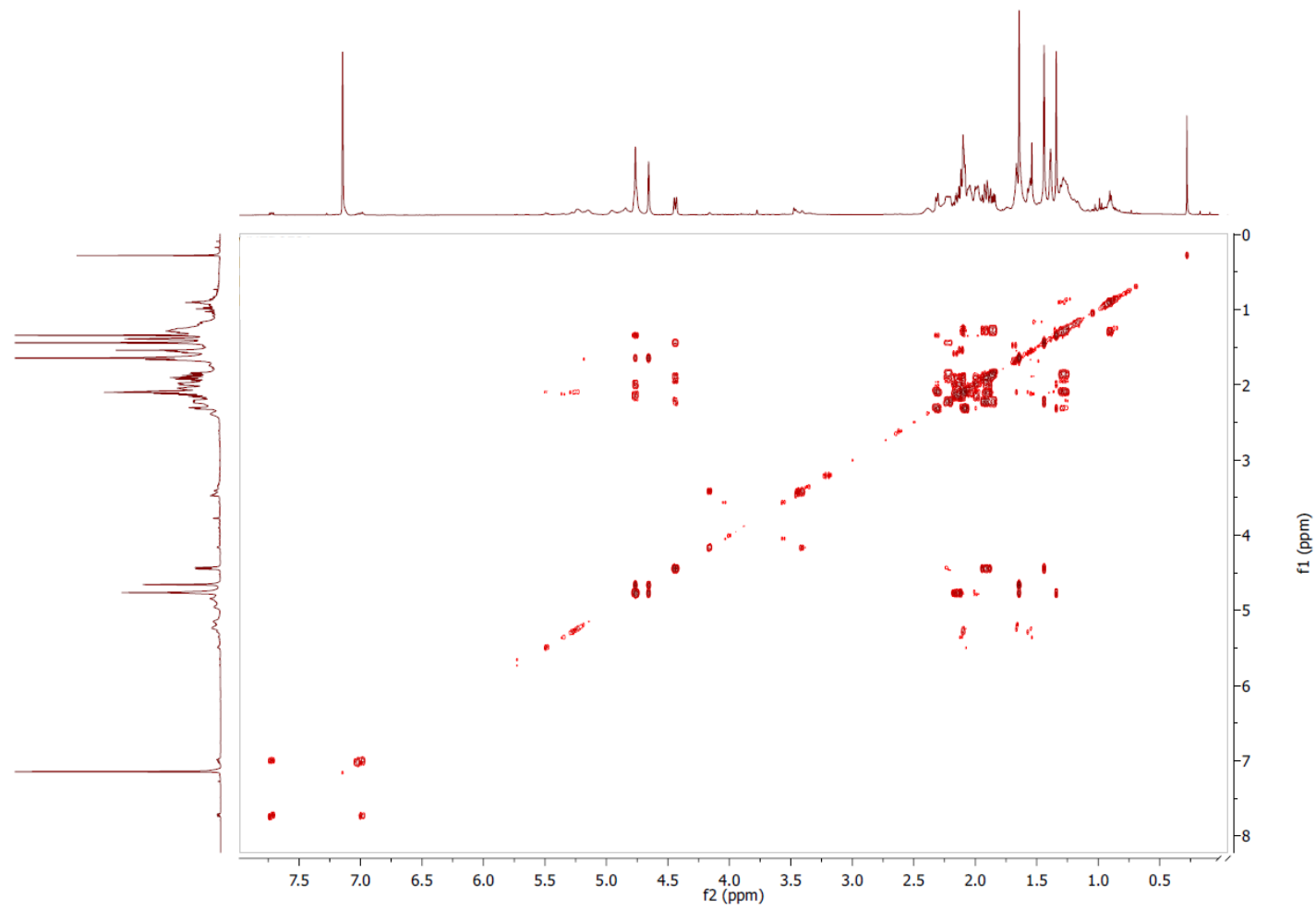

HMBC (28 - $\left.600 \mathrm{MHz}, \mathrm{C}_{6} \mathrm{D}_{6}\right)$

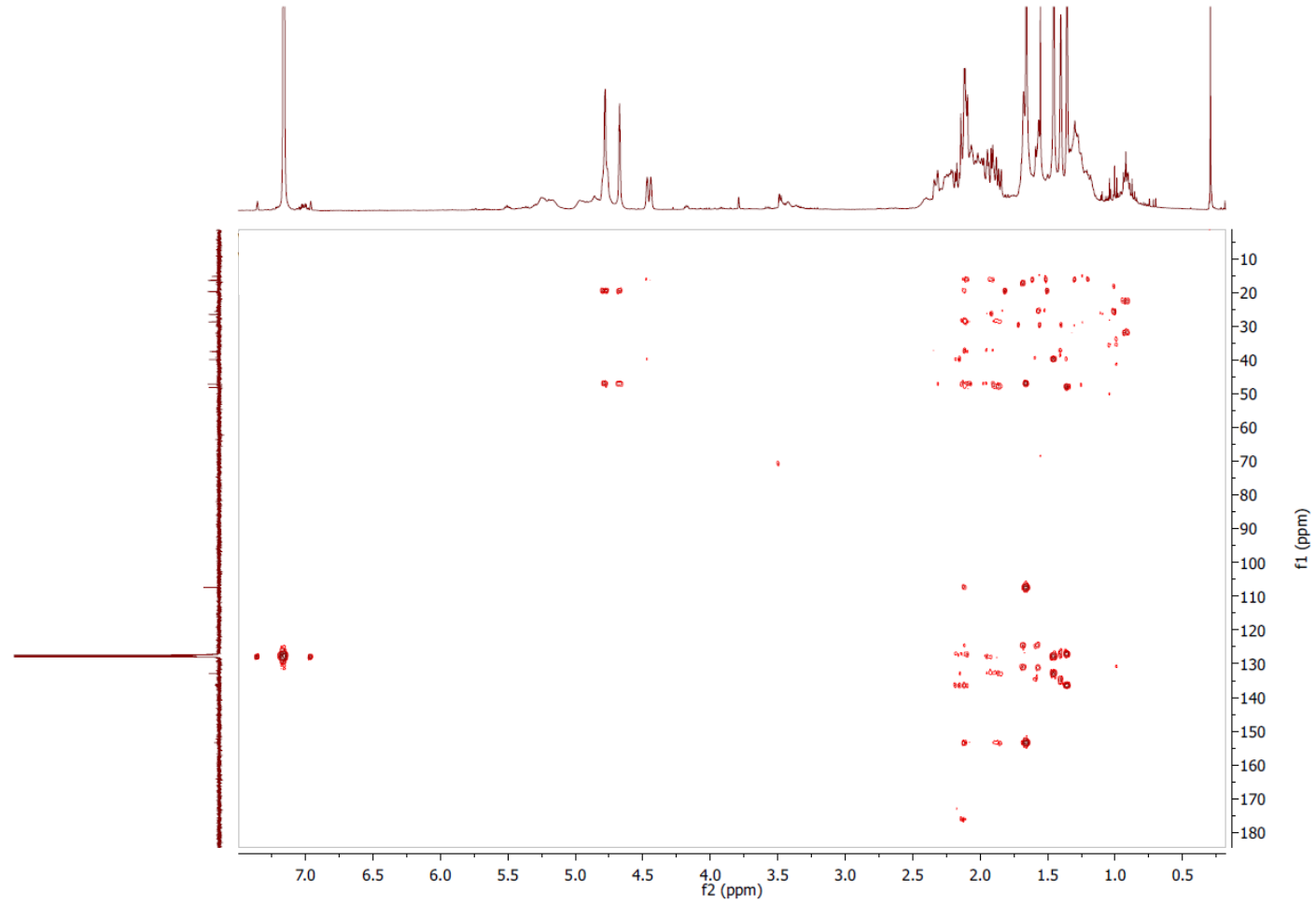


1d-NOE (28 - $\left.600 \mathrm{MHz}, \mathrm{C}_{6} \mathrm{D}_{6}\right)$
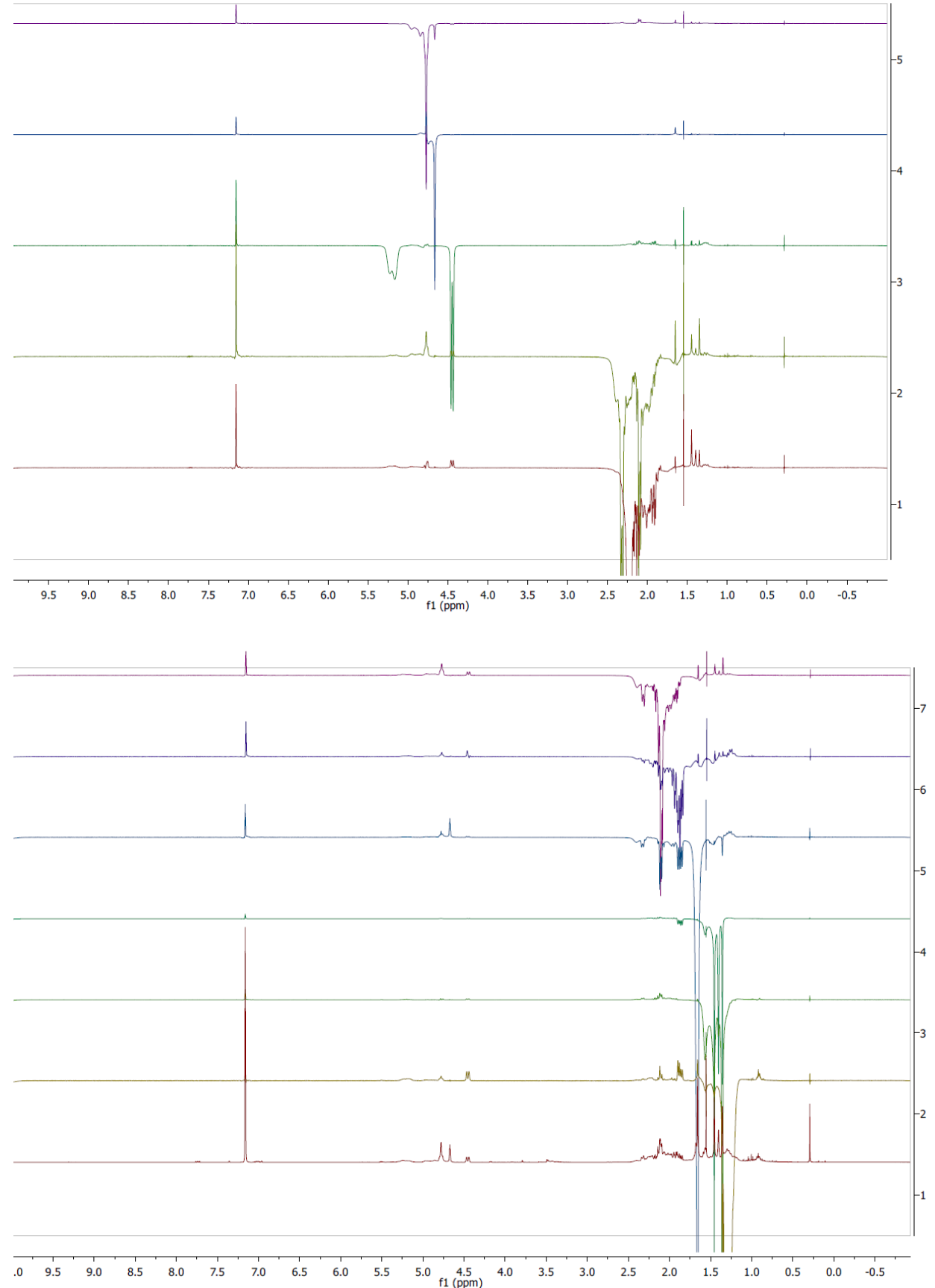

Conformers of 28 at $255 \mathrm{~K}$ in toluene- $\mathrm{d}_{8}$

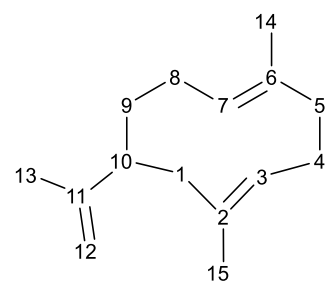<smiles></smiles> 


\begin{tabular}{|c|c|c|c|c|c|c|c|c|}
\hline $\mathrm{Nr}$ & $\delta^{13 c}$ & $\delta^{1 \mathrm{H}}$ & \#H & Multi & $\mathrm{J}(\mathrm{Hz})$ & cosY & HMBC & 1d-noe \\
\hline $\begin{array}{l}1 \mathrm{a} \\
1 \mathrm{~b}\end{array}$ & 48.6 & $\begin{array}{l}2.03 \\
2.26\end{array}$ & 2 & $\begin{array}{l}m \\
d\end{array}$ & 12.4 & $\begin{array}{c}1 b, 15 \\
1 a, 3,15\end{array}$ & $9 b, 10,15$ & \\
\hline 2 & 137.2 & & & & & & $1 a, 1 b, 4 a, 15$ & \\
\hline 3 & 127.7 & 4.69 & 1 & dd & $4.3,11.5$ & $1 b, 4 a, 4 b, 15$ & $\begin{array}{c}1 a, 1 b, 4 b \\
15\end{array}$ & $1 a, 4 a, 5 a, 7$ \\
\hline $\begin{array}{l}4 a \\
4 b\end{array}$ & 27.2 & $\begin{array}{l}1.96 \\
2.10\end{array}$ & 2 & $\begin{array}{l}\mathrm{m} \\
\mathrm{m}\end{array}$ & & $\begin{array}{c}3,5 a, 14 \\
3,5 b\end{array}$ & $3,5 a, 5 b, 14$ & \\
\hline $\begin{array}{l}5 a \\
5 b\end{array}$ & 40.5 & $\begin{array}{l}1.85 \\
2.08\end{array}$ & 2 & $\mathrm{t}$ & 16.3 & $\begin{array}{c}4 a, 4 b, 5 b \\
5 a\end{array}$ & $\begin{array}{c}4 a, 4 b, 7,14 \\
15\end{array}$ & \\
\hline 6 & 128.2 & & & & & & $\begin{array}{c}4 b, 8 a, 8 b \\
14\end{array}$ & \\
\hline 7 & 133.6 & 4.35 & 1 & $d$ & 10.9 & $8 a, 8 b, 14$ & $\begin{array}{c}5 b, 9 a, 9 b \\
14\end{array}$ & $1 a, 3,5 a, 8 b, 9 a$ \\
\hline $\begin{array}{l}8 a \\
8 b\end{array}$ & 29.2 & $\begin{array}{l}1.85 \\
2.18\end{array}$ & 2 & $\begin{array}{l}\text { ddd } \\
\text { dd }\end{array}$ & $\begin{array}{c}2.3,10.9 \\
11.3 \\
7.4,11.3\end{array}$ & $\begin{array}{c}7,8 b, 9 a \\
7,8 a, 9 a, 9 b \\
14\end{array}$ & $\begin{array}{c}7,9 a, 9 b, 10 \\
14\end{array}$ & \\
\hline $\begin{array}{l}9 a \\
9 b\end{array}$ & 37.9 & $\begin{array}{l}1.19 \\
1.79\end{array}$ & 2 & $\begin{array}{c}\mathrm{m} \\
\mathrm{ddd}\end{array}$ & $\begin{array}{c}0.9,7.4 \\
14.2\end{array}$ & $\begin{array}{c}8 a, 8 b, 9 b, 10 \\
9 a, 10\end{array}$ & $\begin{array}{c}1 a, 1 b, 7,10 \\
12 b\end{array}$ & $\begin{array}{c}1 a, 7,9 b, 12 b, 13 \\
8 b, 9 a, 12 a, 12 b \\
13\end{array}$ \\
\hline 10 & 47.5 & 2.08 & 1 & $\mathrm{~m}$ & & $9 a, 9 b$ & $\begin{array}{c}1 a, 1 b, 12 a \\
12 b, 13\end{array}$ & \\
\hline 11 & 154.0 & & & & & & $\begin{array}{c}1 b, 9 b, 10 \\
12 a, 12 b, 13\end{array}$ & \\
\hline $\begin{array}{l}12 a \\
12 b\end{array}$ & 108.1 & $\begin{array}{l}4.64 \\
4.75\end{array}$ & 2 & $\begin{array}{l}\text { dd } \\
\text { dd }\end{array}$ & $\begin{array}{l}1.4,2.0 \\
0.7,1.4\end{array}$ & $\begin{array}{l}12 b, 13 \\
12 b, 13\end{array}$ & 10,13 & \\
\hline 13 & 20.3 & 1.63 & 3 & $d d$ & $0.7,1.4$ & $12 a, 12 b$ & $10,12 a, 12 b$ & \\
\hline 14 & 17.2 & 1.41 & 3 & $\mathrm{t}$ & 1.6 & $4 a, 7,8 b$ & $5 a, 5 b, 7$ & $4 b, 8 a, 15$ \\
\hline 15 & 17.1 & 1.29 & 3 & s & & $1 a, 1 b, 3$ & $1 a, 1 b, 3$ & $\begin{array}{c}1 b, 4 b, 8 a, 10 \\
12 b, 14\end{array}$ \\
\hline
\end{tabular}
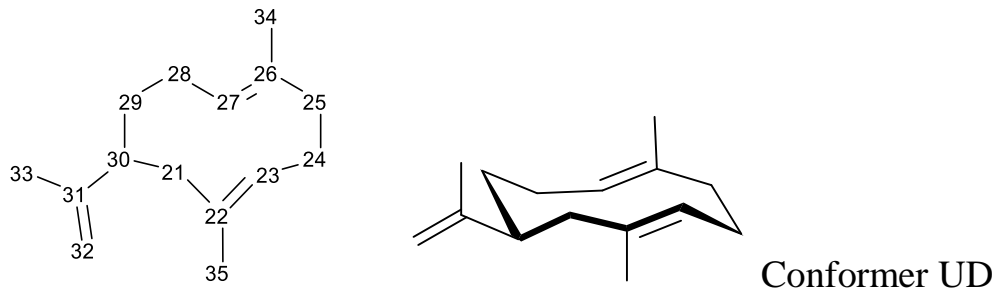

\begin{tabular}{|c|c|c|c|c|c|c|c|c|}
\hline $\mathrm{Nr}$ & $\delta^{13 c}$ & $\delta^{1 \mathrm{H}}$ & $\# \mathbf{H}$ & Multi & $\mathrm{J}(\mathrm{Hz})$ & cosy & HMBC & 1D- noe \\
\hline $21 a$ & 47.8 & 1.88 & 2 & $\mathrm{t}$ & 8.0 & $21 a, 35$ & $23,29 a, 35$ & \\
\hline $21 b$ & & 1.97 & & d & 11.4 & $21 b, 35$ & & \\
\hline 22 & 137.3 & & & & & & $21 a, 24 b, 35$ & \\
\hline 23 & 122.9 & 4.89 & 1 & $\mathrm{t}$ & 8.0 & $21 a, 24 a, 24 b, 35$ & $21 a, 24 a$ & $21 a, 24 a, 29$ \\
\hline
\end{tabular}




\begin{tabular}{|c|c|c|c|c|c|c|c|c|}
\hline & & & & & & & $24 b, 25 a, 35$ & 34 \\
\hline $\begin{array}{l}24 a \\
24 b\end{array}$ & $\begin{array}{c}24.9 / \\
25.0\end{array}$ & $\begin{array}{l}2.20 \\
1.69\end{array}$ & 2 & $\begin{array}{l}\mathrm{m} \\
\mathrm{m}\end{array}$ & & $\begin{array}{c}23,24 b \\
23,24 a, 25 a, 25 b\end{array}$ & $\begin{array}{c}23,25 a, 25 b, \\
35\end{array}$ & \\
\hline $\begin{array}{l}25 a \\
25 b\end{array}$ & 38.0 & $\begin{array}{l}2.01 \\
2.08\end{array}$ & 2 & $\begin{array}{l}d \\
d\end{array}$ & $\begin{array}{c}10.9 \\
9.3\end{array}$ & $\begin{array}{l}24 b, 25 b, 34 \\
24 b, 25 a, 27\end{array}$ & $\begin{array}{c}24 a, 24 b, 27, \\
28 a, 34\end{array}$ & \\
\hline 26 & & & & & & & $24 b, 28 a, 34$ & \\
\hline 27 & 127.2 & 5.09 & 1 & $d d$ & $6.6,9.4$ & $28 a, 28 b, 34$ & $28 a, 29 b, 34$ & $\begin{array}{c}25 b, 28 a, 29 b \\
30,35\end{array}$ \\
\hline $\begin{array}{l}28 a \\
28 b\end{array}$ & 28.0 & $\begin{array}{l}1.69 \\
2.24\end{array}$ & 2 & $\begin{array}{c}\mathrm{dtd} \\
\mathrm{m}\end{array}$ & $3.8,6.6,10.7$ & $\begin{array}{c}28 b, 29 a, 34 \\
27,28 a, 29 a, 29 b\end{array}$ & $\begin{array}{c}27,29 a, 30 \\
34\end{array}$ & $27,28 b, 29 b, 30$ \\
\hline $\begin{array}{l}29 a \\
29 b\end{array}$ & 35.6 & $\begin{array}{l}1.13 \\
1.55\end{array}$ & 2 & $\begin{array}{l}\mathrm{tt} \\
\mathrm{m}\end{array}$ & $3.4,13.6$ & $\begin{array}{l}28 a, 28 b, 29 b, 30 \\
28 a, 28 b, 29 a, 30\end{array}$ & $\begin{array}{c}21 a, 21 b, \\
28 a, 28 b, 34\end{array}$ & \\
\hline 30 & 45.3 & 1.93 & 1 & $d$ & 11.4 & $29 a, 29 b$ & $\begin{array}{c}21 a, 21 b, 23 \\
28 b, 32 a \\
32 b, 33,35\end{array}$ & \\
\hline 31 & 152.9 & & & & & & $\begin{array}{c}21 a, 32 a \\
32 b, 33\end{array}$ & \\
\hline $\begin{array}{l}32 a \\
32 b\end{array}$ & 108.2 & $\begin{array}{l}4.64 \\
4.75\end{array}$ & 2 & $\begin{array}{c}d d \\
t\end{array}$ & $\begin{array}{c}1.4,2.4 \\
2.4\end{array}$ & $\begin{array}{l}32 b, 33 \\
32 a, 33\end{array}$ & 30,33 & \\
\hline 33 & 20.2 & 1.61 & 3 & s & & $32 a, 32 b$ & $32 a, 32 b$ & \\
\hline 34 & 15.8 & 1.35 & 3 & s & & $25 a, 27,28 a$ & $25 b, 27$ & $\begin{array}{c}23,25 a, 28 b \\
29 a\end{array}$ \\
\hline 35 & 17.4 & 1.40 & 3 & s & & $21 a, 21 b, 23$ & $21 a, 21 b, 23$ & $\begin{array}{c}21 b, 24 b, 25 b \\
27,30\end{array}$ \\
\hline
\end{tabular}
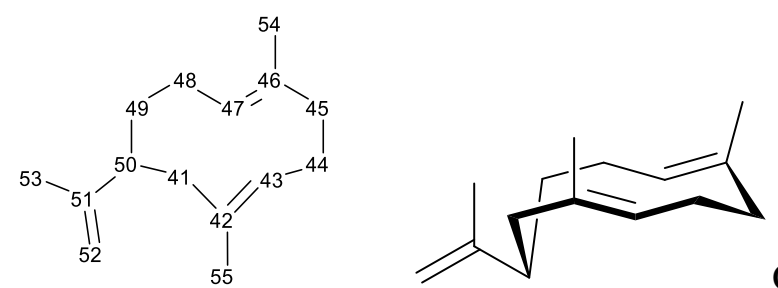

Conformer UU

\begin{tabular}{|c|c|c|c|c|c|c|c|c|}
\hline $\mathrm{Nr}$ & $\delta^{13 c}$ & $\delta^{1 \mathrm{H}}$ & $\# \mathbf{H}$ & Multi & $\mathrm{J}(\mathrm{Hz})$ & $\cos Y$ & HMBC & 1d-noe \\
\hline $\begin{array}{l}41 a \\
41 b\end{array}$ & 40,7 & $\begin{array}{l}1,58 \\
2,32\end{array}$ & 2 & $\begin{array}{l}\mathrm{t} \\
\mathrm{m}\end{array}$ & 10.2 & $\begin{array}{c}41 b \\
41 a, 43\end{array}$ & $\begin{array}{c}43,44 b, 49 a, 50 \\
55\end{array}$ & $\begin{array}{c}41 b, 52 a, 52 b \\
52 b, 53\end{array}$ \\
\hline 42 & 133,8 & & & & & & $\begin{array}{c}41 a, 41 b, 44 a \\
44 b, 55\end{array}$ & \\
\hline 43 & 129,7 & 4,76 & 1 & $\mathrm{~m}$ & & $41 a, 44 a, 44 b, 55$ & $41 a, 41 b, 44 b, 45 a$ & $44 b$ \\
\hline $\begin{array}{l}44 a \\
44 b\end{array}$ & $\begin{array}{c}25,0 / \\
24,9\end{array}$ & $\begin{array}{l}1,92 \\
2,20\end{array}$ & 2 & $\begin{array}{l}\mathrm{m} \\
\mathrm{m}\end{array}$ & & $\begin{array}{c}43,44 b \\
43,44 a, 45 a, 45 b\end{array}$ & $41 b, 45 a, 45 b, 54$ & \\
\hline $\begin{array}{l}45 a \\
45 b\end{array}$ & 39,2 & $\begin{array}{l}1,96 \\
2,02\end{array}$ & 2 & $\begin{array}{l}\mathrm{m} \\
\mathrm{d}\end{array}$ & 3.3 & $\begin{array}{c}44 b \\
44 b, 54\end{array}$ & $44 b, 47,54,55$ & \\
\hline 46 & 136,3 & & & & & & $45 a, 48 a, 54$ & \\
\hline 47 & 128,4 & 5,18 & 1 & $\mathrm{t}$ & 7.8 & $48 a, 48 b, 54$ & $45 a, 49 a, 54$ & $\begin{array}{c}43,45 a, 48 b \\
49 b, 50\end{array}$ \\
\hline
\end{tabular}




\begin{tabular}{|c|c|c|c|c|c|c|c|c|}
\hline $\begin{array}{l}48 a \\
48 b\end{array}$ & 25,9 & $\begin{array}{l}1,80 \\
2,13\end{array}$ & 2 & $\begin{array}{l}\mathrm{m} \\
\mathrm{td}\end{array}$ & $2.2,4.4$ & $\begin{array}{l}47,48 b, 49 a, 49 b \\
47,48 a, 49 a, 49 b\end{array}$ & $47,49 a, 50$ & $48 b, 47$ \\
\hline $\begin{array}{l}49 a \\
49 b\end{array}$ & 32,8 & $\begin{array}{l}1,18 \\
1,37\end{array}$ & 2 & $\begin{array}{l}\mathrm{m} \\
\mathrm{m}\end{array}$ & & $\begin{array}{c}48 a, 48 b, 49 b \\
48 a, 48 b, 49 a, 50\end{array}$ & $\begin{array}{c}41 a, 41 b, 48 a, 50 \\
54\end{array}$ & \\
\hline 50 & 47,6 & 2,33 & 1 & $\mathrm{~m}$ & & $\begin{array}{c}49 a, 49 b, 52 a \\
52 b\end{array}$ & $\begin{array}{c}41 a, 41 b, 48 b \\
49 a, 52 a, 52 b, 53\end{array}$ & $\begin{array}{c}41 a, 43,47 \\
52 a, 52 b\end{array}$ \\
\hline 51 & 151,1 & & & & & & $41 b, 52 a, 52 b, 53$ & \\
\hline $\begin{array}{l}52 a \\
52 b\end{array}$ & 109,3 & $\begin{array}{l}4,74 \\
4,84\end{array}$ & 2 & $\begin{array}{l}\mathrm{t} \\
\mathrm{d}\end{array}$ & $\begin{array}{l}2.4 \\
2.4\end{array}$ & $\begin{array}{l}52 b, 53 \\
52 a, 53\end{array}$ & 50,53 & \\
\hline 53 & 20,9 & 1,66 & 3 & $\mathrm{~m}$ & & $52 a, 52 b$ & $41 b, 52 b$ & \\
\hline 54 & 15,8 & 1,35 & 3 & s & & $45 a, 47$ & $45 a, 47$ & $\begin{array}{c}41 b, 45 b, \\
48 b, 49 a, 53 \\
55\end{array}$ \\
\hline 55 & 22,3 & 1,53 & 3 & $\mathrm{~s}$ & & 43 & $41 a, 41 b, 43$ & \\
\hline
\end{tabular}

${ }^{1}$ H-NMR (28 - $600 \mathrm{MHz}$, toluene-d 8 )

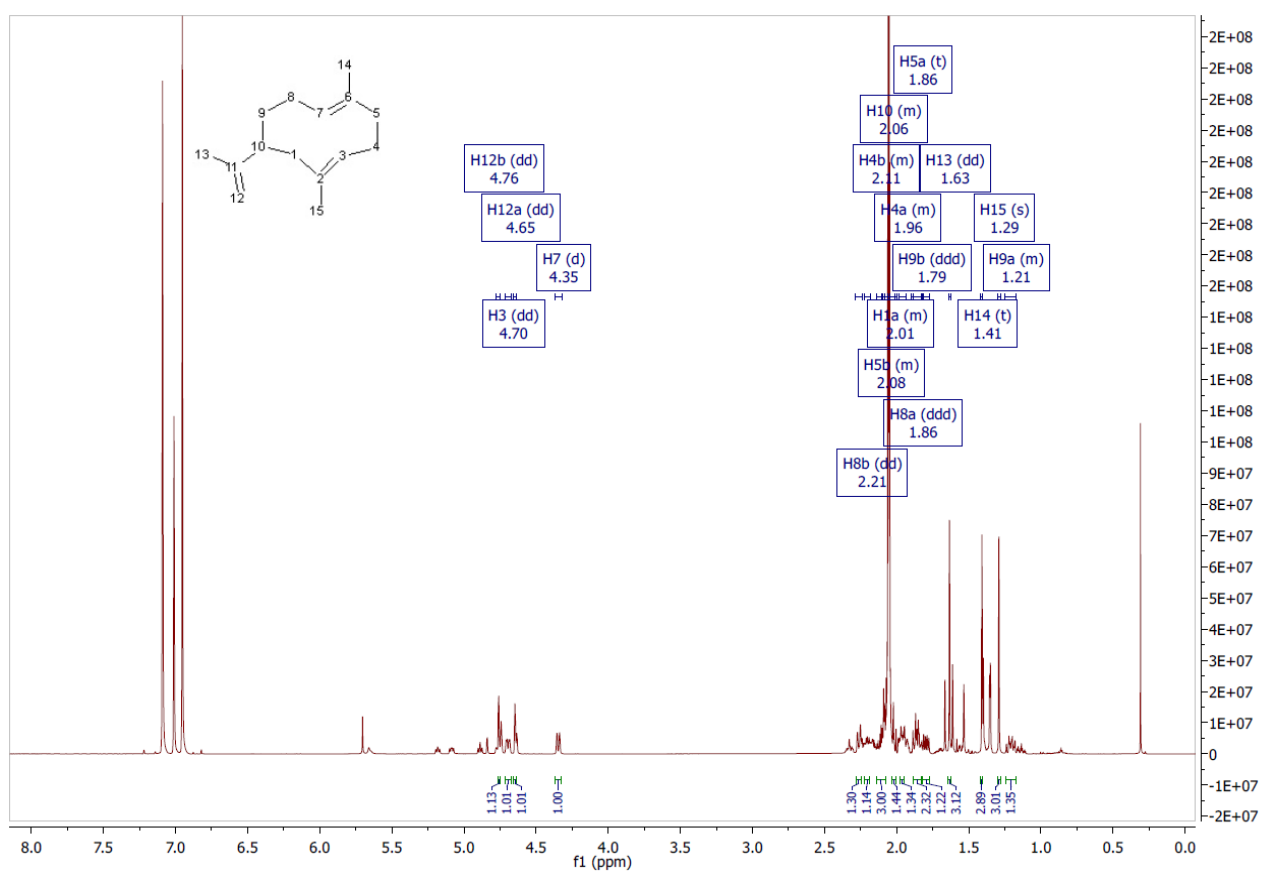




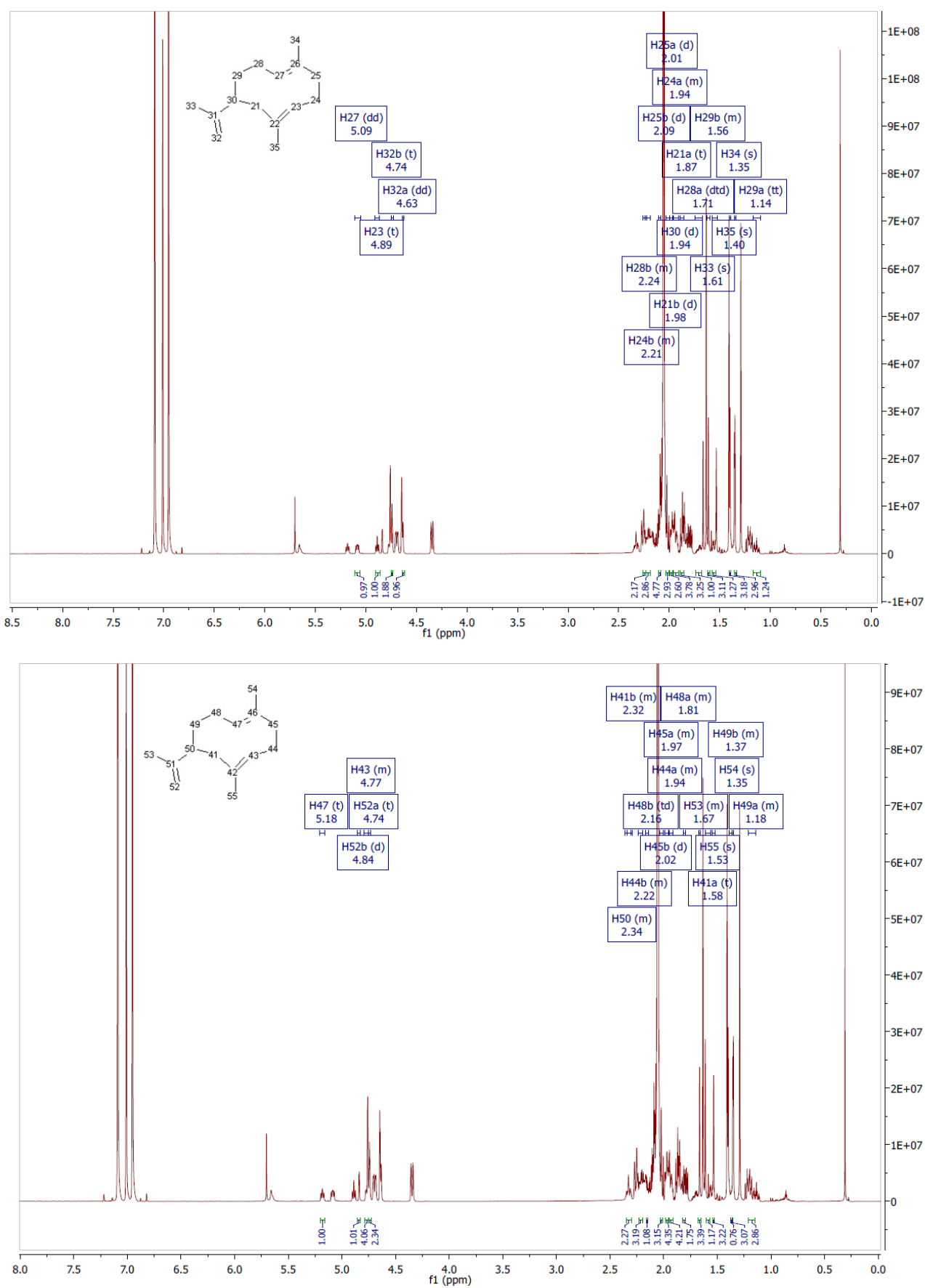


${ }^{13} \mathrm{C}-\mathrm{NMR}\left(\mathbf{2 8}-151 \mathrm{MHz}\right.$, toluene- $\left.\mathrm{d}_{8}\right)$

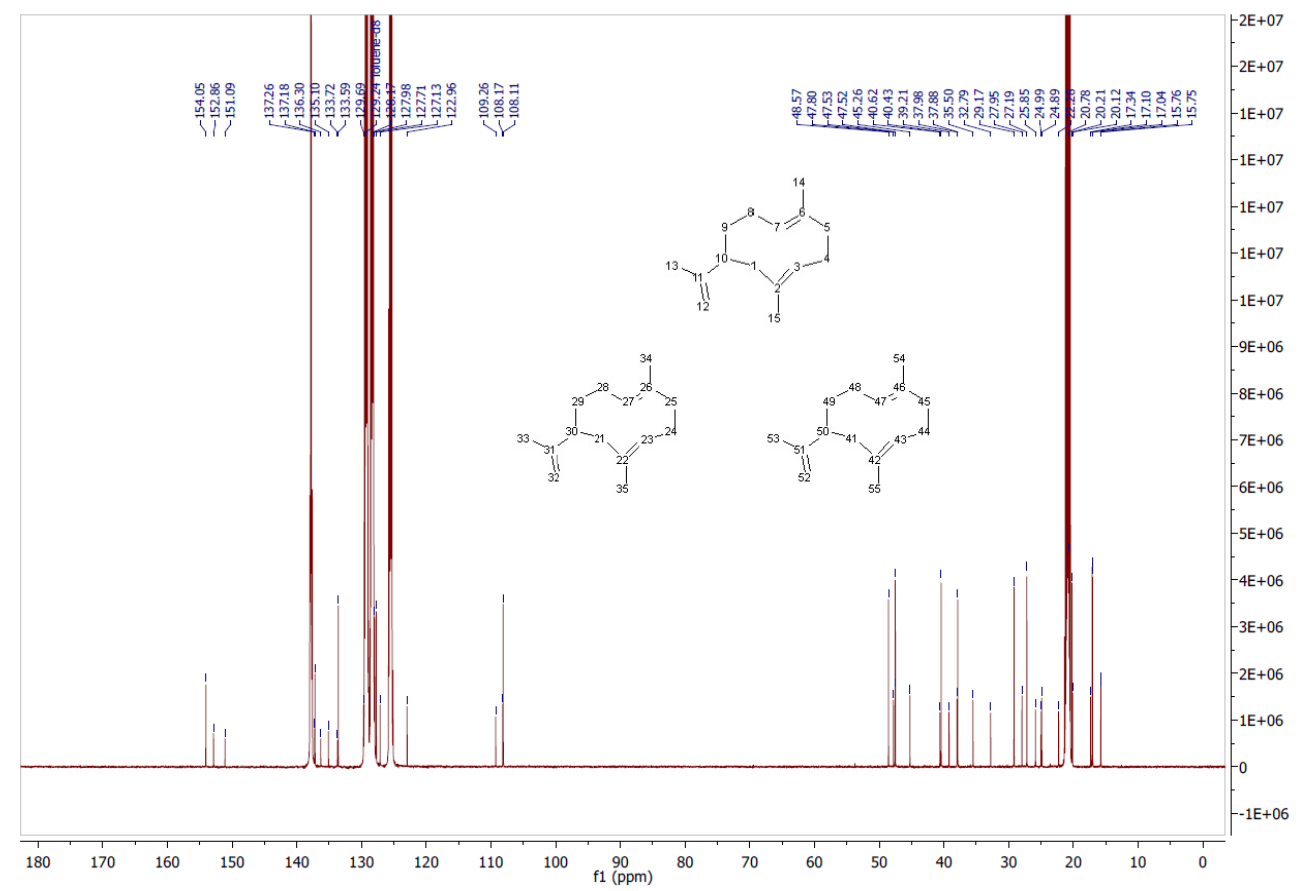

HSQC (28 - $600 \mathrm{MHz}$, toluene- $\left.\mathrm{d}_{8}\right)$

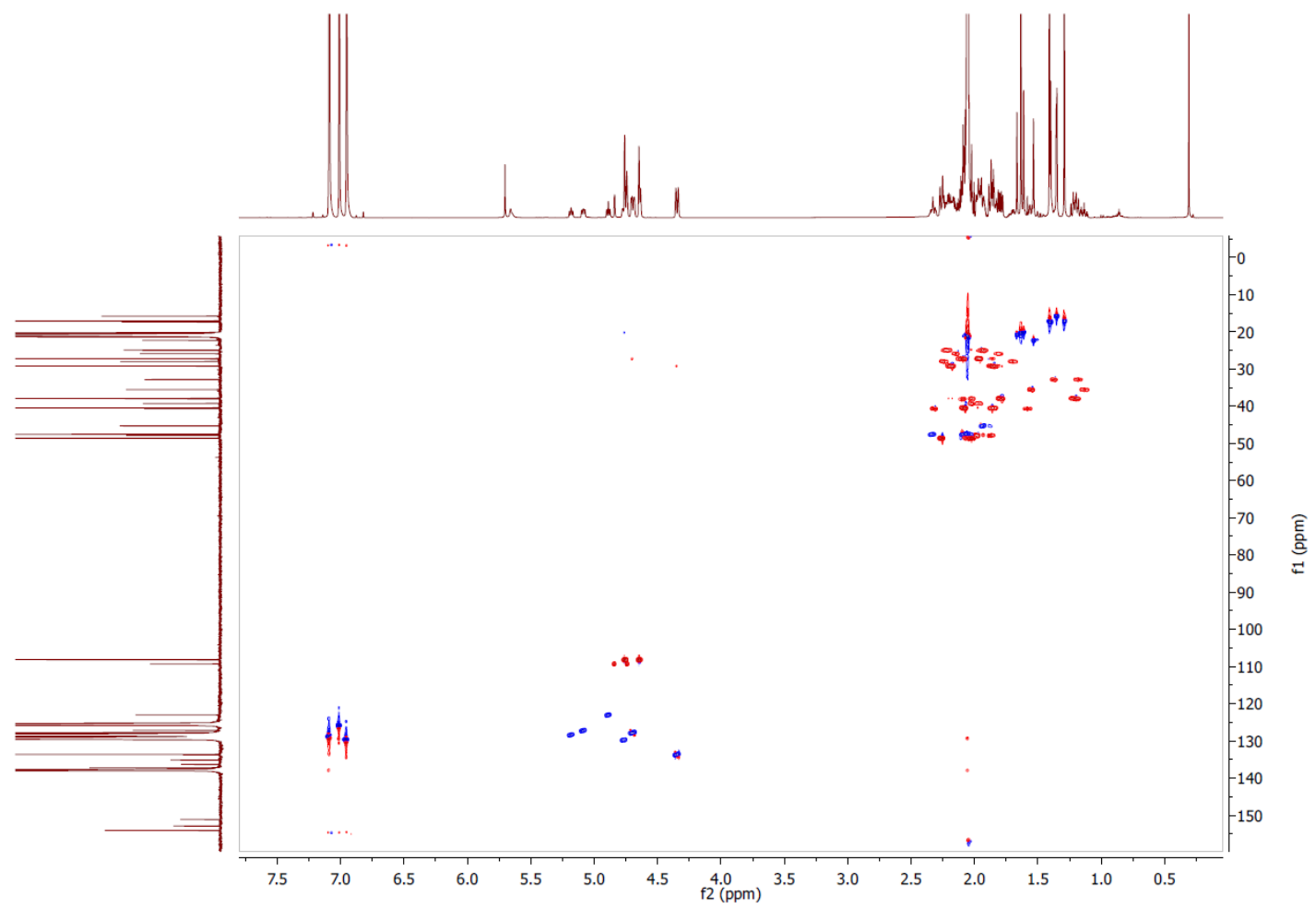


COSY $\left(28-600 \mathrm{MHz}\right.$, toluene- $\left.\mathrm{d}_{8}\right)$

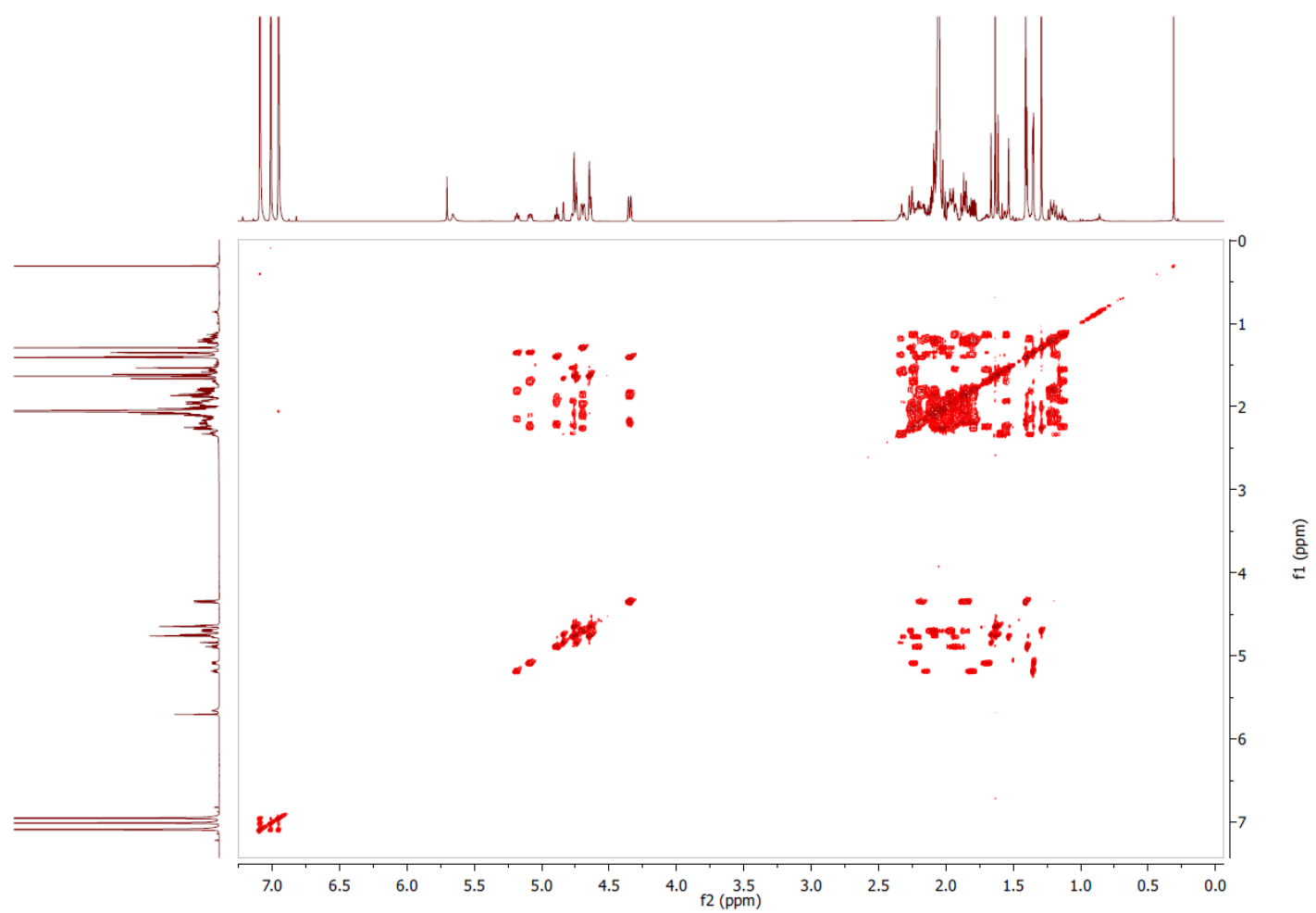

HMBC $\left(28-600 \mathrm{MHz}\right.$, toluene- $\left.\mathrm{d}_{8}\right)$

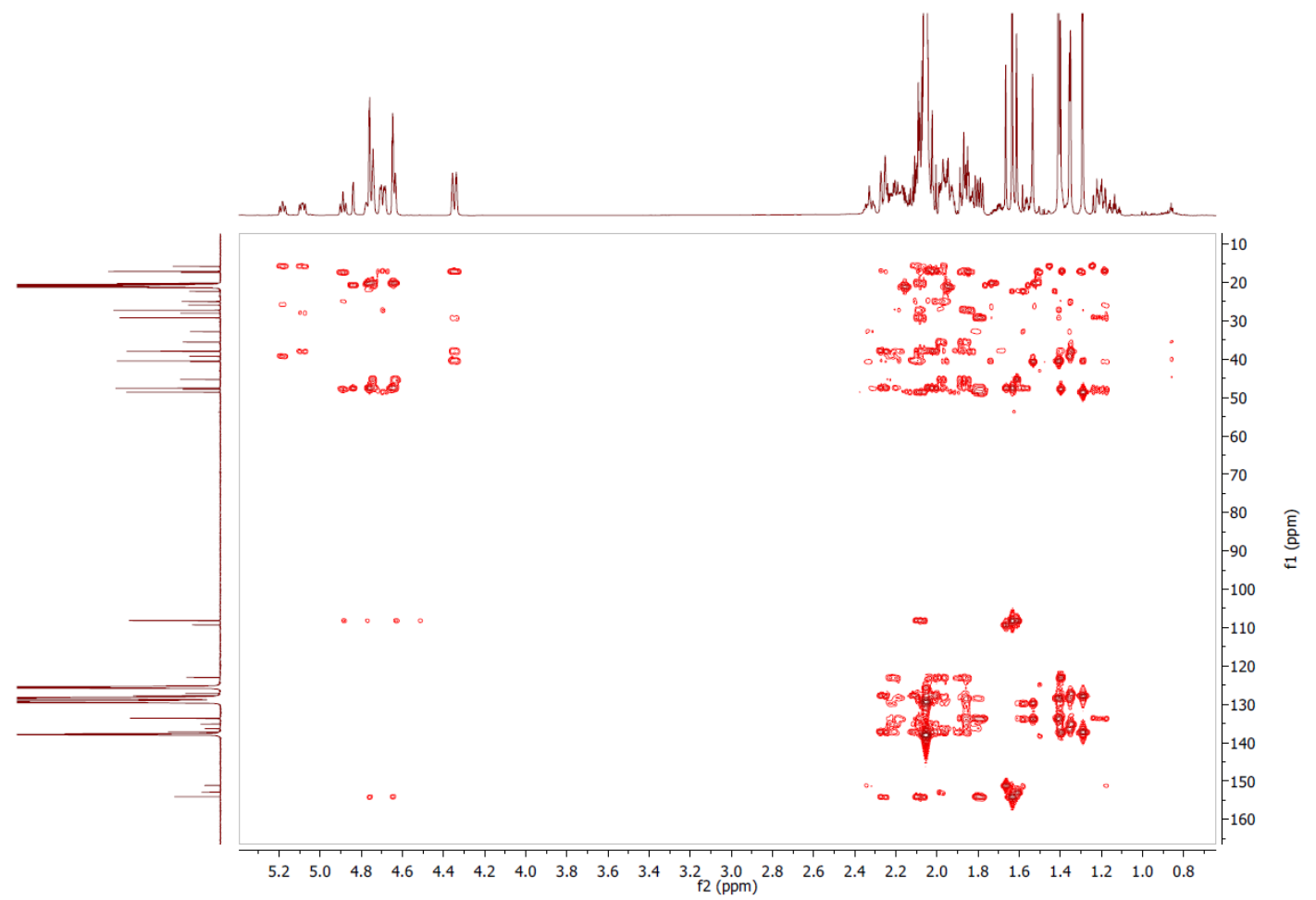


1d-NOE (28 - $600 \mathrm{MHz}$, toluene-d 8 )
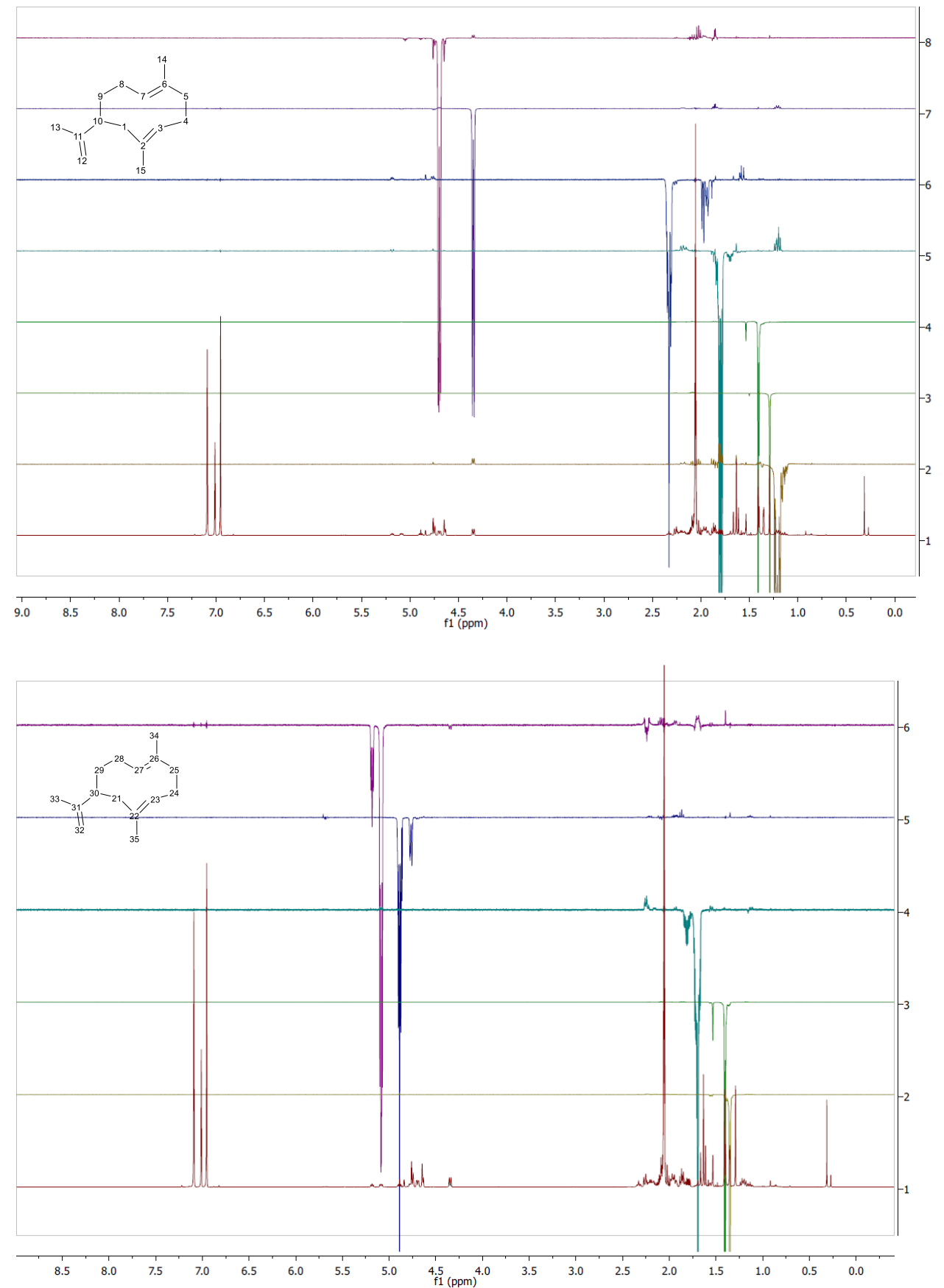


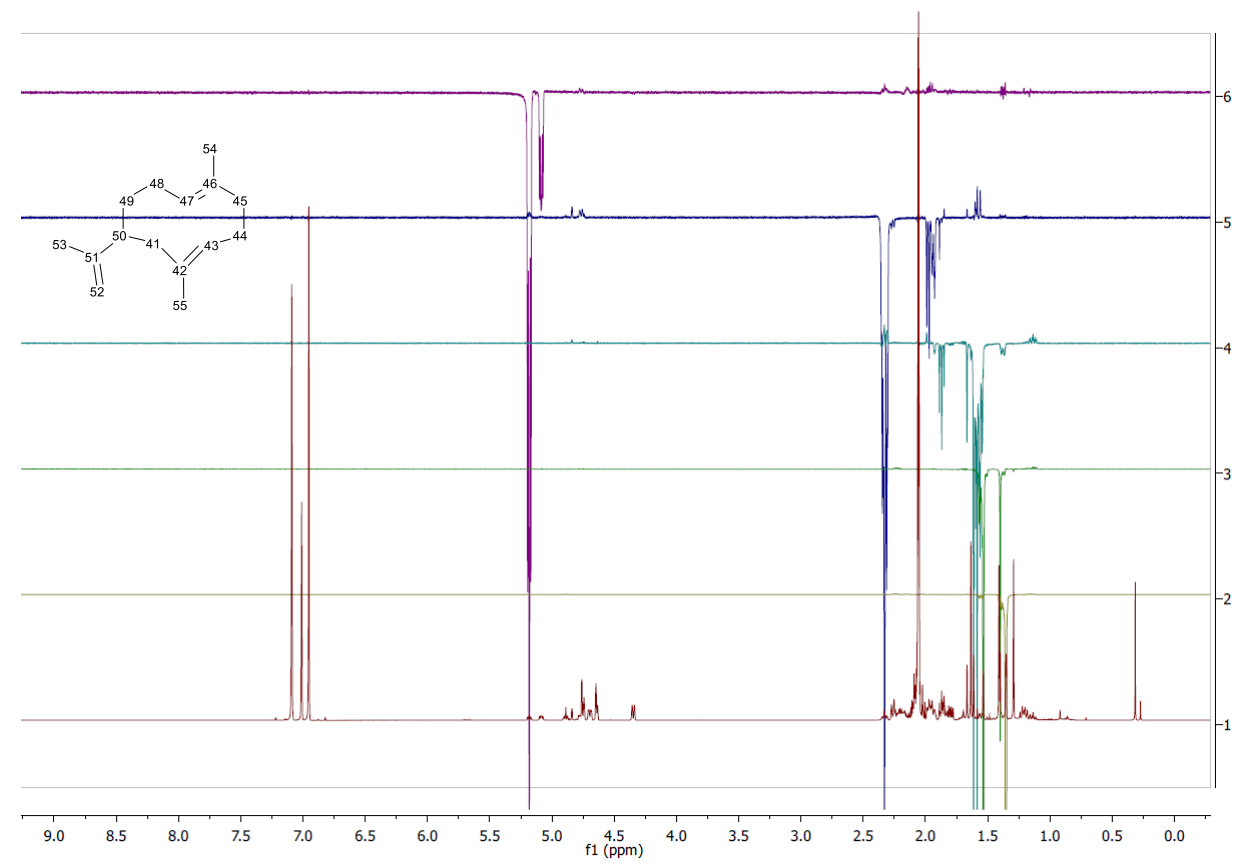

\subsubsection{Energy calculations for conformers of 28}

Energy calculations were performed with Schrödinger Maestro 9.8 using the software module 'conformational search' with MM3 force field and a dielectric constant of 2.4 for toluene. 18.000 starting structures were generated and optimized to identify 44 indepentant conformers. The best 15 conformers were analysed and the fractional population distribution was calculated based on the Boltzmann distribution.

Table S5: 15 most likely conformations of 28 at $255 \mathrm{~K}$, solved in toluene. Possible conformers were assigned to the four conformers (DD, UD, UU and DU), whereby the orientation of the isopropylen group varies for each calculated conformation

\section{structure Calculated G Population distribution of conformer $[\mathrm{kJ} / \mathrm{mol}] \quad$ conformers [\%]}

\begin{tabular}{|cccc}
\hline $\mathbf{1}$ & 132,83 & 89,01 & DD \\
\hline $\mathbf{2}$ & 139,46 & 3,90 & UD \\
$\mathbf{3}$ & 140,24 & 2,70 & DD \\
$\mathbf{4}$ & 140,87 & 2,00 & DD \\
$\mathbf{5}$ & 143,08 & 0,71 & DD \\
$\mathbf{6}$ & 143,31 & 0,63 & UU \\
$\mathbf{7}$ & 144,23 & 0,41 & UD \\
$\mathbf{8}$ & 144,83 & 0,31 & UU \\
$\mathbf{9}$ & 146,63 & 0,13 & DU \\
$\mathbf{1 0}$ & 147,48 & 0,09 & UD \\
$\mathbf{1 1}$ & 147,65 & 0,08 & UD \\
\hline $\mathbf{1 2}$ & 152,05 & 0,01 & DU \\
$\mathbf{1 3}$ & 152,10 & 0,01 & UU \\
\hline $\mathbf{1 4}$ & 152,19 & 0,01 & UU \\
$\mathbf{1 5}$ & 153,38 & 0,01 & DD \\
\hline
\end{tabular}

\subsubsection{COPE rearrangement product 31}

HR-GC-MS: exact mass: 204.1878 found: 204.1878 


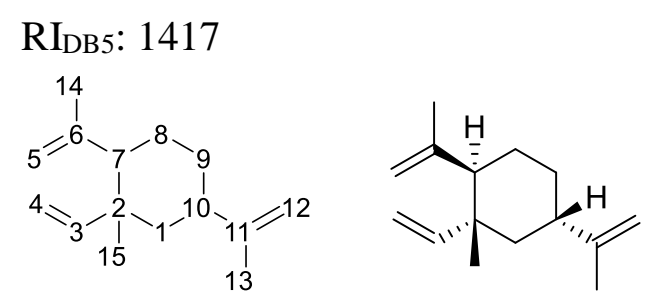

\begin{tabular}{|c|c|c|c|c|c|c|c|c|}
\hline $\mathrm{Nr}$ & $\delta^{13 C}$ & $\delta^{1 \mathrm{H}}$ & \#H & Multi & $\mathrm{J}(\mathrm{Hz})$ & COSY & HМBC & NOESY \\
\hline $\begin{array}{l}1 a_{a x} \\
1 b_{e q}\end{array}$ & 45.5 & $\begin{array}{l}1.31 \\
1.49\end{array}$ & 2 & $\begin{array}{c}\mathrm{t} \\
\mathrm{dd}\end{array}$ & $\begin{array}{c}12.7 \\
3.1,13.1\end{array}$ & $\begin{array}{c}1 b, 10,15 \\
1 a, 10\end{array}$ & $\begin{array}{c}3,4 a, 4 b, 7 \\
9 a, 10,15\end{array}$ & $\begin{array}{c}1 b, 3,4 a, 4 b, 7,12,13 \\
1 a, 8,9 a, 10,12\end{array}$ \\
\hline 2 & 40.8 & & & & & & $3,4 a, 4 b, 7,15$ & \\
\hline 3 & 150.6 & 5.77 & 1 & dd & $\begin{array}{l}10.8 \\
17.5\end{array}$ & $4 a, 4 b$ & $\begin{array}{c}1 a, 4 a, 4 b, 7 \\
15\end{array}$ & $1 a, 4 a, 7$ \\
\hline $\begin{array}{l}4 a \\
4 b\end{array}$ & 110.0 & $\begin{array}{l}4.90 \\
4.93\end{array}$ & 2 & $\begin{array}{l}\text { dd } \\
\text { dd }\end{array}$ & $\begin{array}{l}10.8,1.3 \\
17.5,1.3\end{array}$ & $\begin{array}{l}3 \\
3\end{array}$ & 15 & $\begin{array}{c}1 a, 3 \\
1 a, 14,15\end{array}$ \\
\hline $\begin{array}{l}5 a \\
5 b\end{array}$ & 112.5 & $\begin{array}{l}4.68 \\
4.93\end{array}$ & 2 & $\begin{array}{l}\mathrm{m} \\
\mathrm{dd}\end{array}$ & $1.0,2.5$ & $\begin{array}{c}5 b, 14 \\
5 a, 7,14\end{array}$ & 7,14 & $\begin{array}{c}5 b, 7,8 \\
5 a, 14\end{array}$ \\
\hline 6 & 147.7 & & & & & & $5 a, 5 b, 7,14$ & \\
\hline 7 & 52.7 & 1.80 & 1 & dd & $4.2,12.2$ & $5 b, 8$ & $\begin{array}{c}1 a, 1 b, 3,5 a \\
5 b, 8,9 a, 9 b \\
14,15\end{array}$ & $1 a, 3,5 a, 8,13$ \\
\hline 8 & 28.1 & 1.51 & 2 & $\mathrm{~m}$ & & $7,9 a, 9 b$ & $7,9 a$ & $1 b, 5 a, 7,9 a, 9 b, 15$ \\
\hline $\begin{array}{l}9 a_{\text {eq }} \\
9 b_{a x}\end{array}$ & 32.2 & $\begin{array}{l}1.10 \\
1.72\end{array}$ & 2 & $\begin{array}{l}\text { ddd } \\
\mathrm{m}\end{array}$ & $4.4,12.5$ & $\begin{array}{l}8,9 b, 10 \\
8,9 a, 10\end{array}$ & $1 a, 1 b, 7,8,10$ & $\begin{array}{c}1 b, 7,8,9 b, 10,12 \\
9 a\end{array}$ \\
\hline 10 & 40.4 & 2.05 & 1 & $\mathrm{tt}$ & $3.1,12.7$ & $\begin{array}{c}1 a, 1 b \\
9 a, 9 b, 12\end{array}$ & $\begin{array}{c}1 a, 1 b, 9 a, 9 b \\
12\end{array}$ & $1 b, 9 a, 12,14,15$ \\
\hline 11 & 150.2 & & & & & & $\begin{array}{c}1 b, 9 a, 10,12 \\
13\end{array}$ & \\
\hline 12 & 108.9 & 4.81 & 2 & $\mathrm{~m}$ & & 10,13 & 10,13 & $1 a, 1 b, 9 a, 10,13$ \\
\hline 13 & 21.1 & 1.65 & 3 & $\mathrm{t}$ & 0.9 & 12 & 10,13 & $1 a, 7,12$ \\
\hline 14 & 25.2 & 1.71 & 3 & dd & $0.8,1.4$ & $5 a, 5 b$ & $5 a, 5 b, 7$ & $4 b, 5 b, 10,15$ \\
\hline 15 & 17.2 & 0.99 & 3 & $\mathrm{~s}$ & & $1 a$ & $1 a, 1 b, 3,7$ & $1 b, 4 a, 4 b, 8,10,14$ \\
\hline
\end{tabular}


${ }^{1} \mathrm{H}-\mathrm{NMR}(\mathbf{3 1})-500 \mathrm{MHz}, \mathrm{C}_{6} \mathrm{D}_{6}$

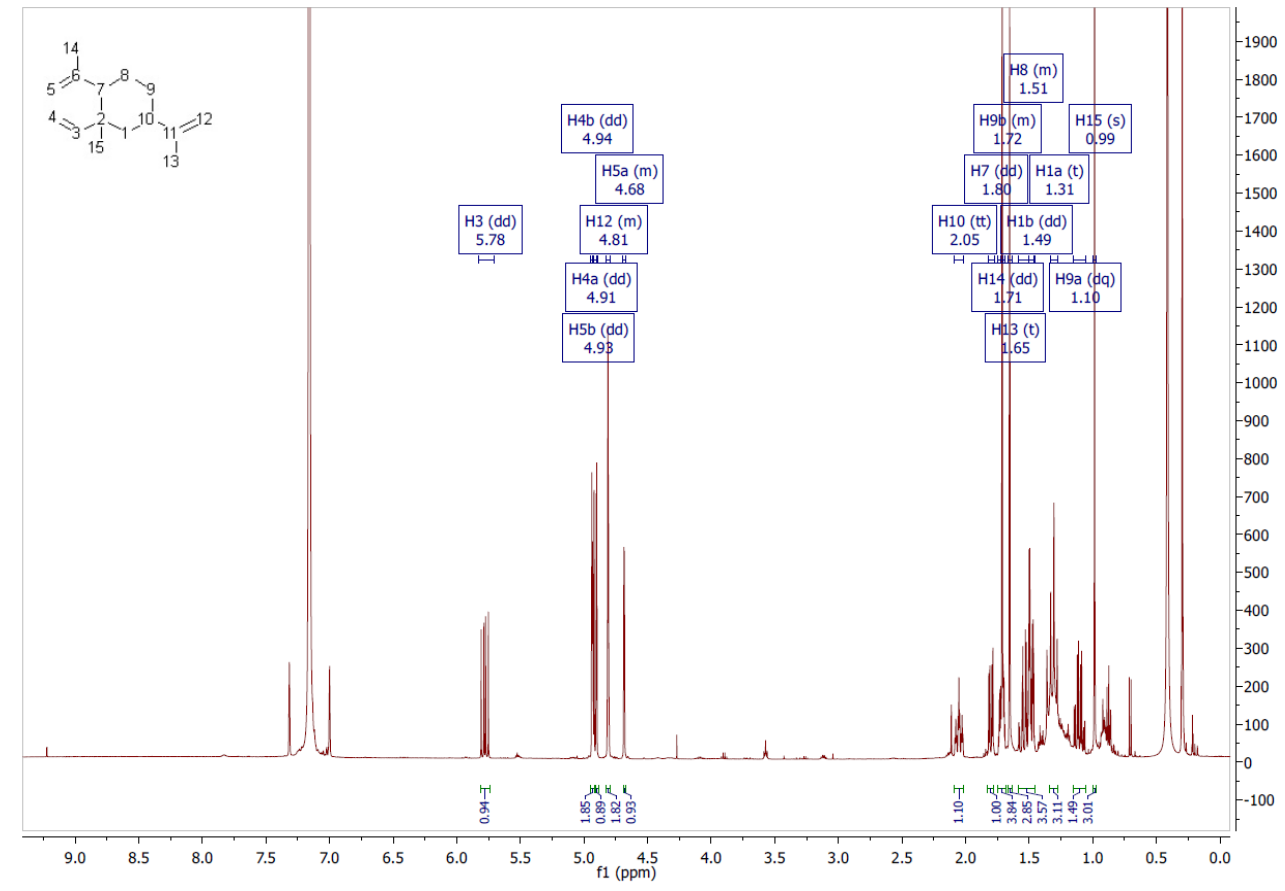

${ }^{13} \mathrm{C}-\mathrm{NMR}(\mathbf{3 1})-121 \mathrm{MHz}, \mathrm{C}_{6} \mathrm{D}_{6}$

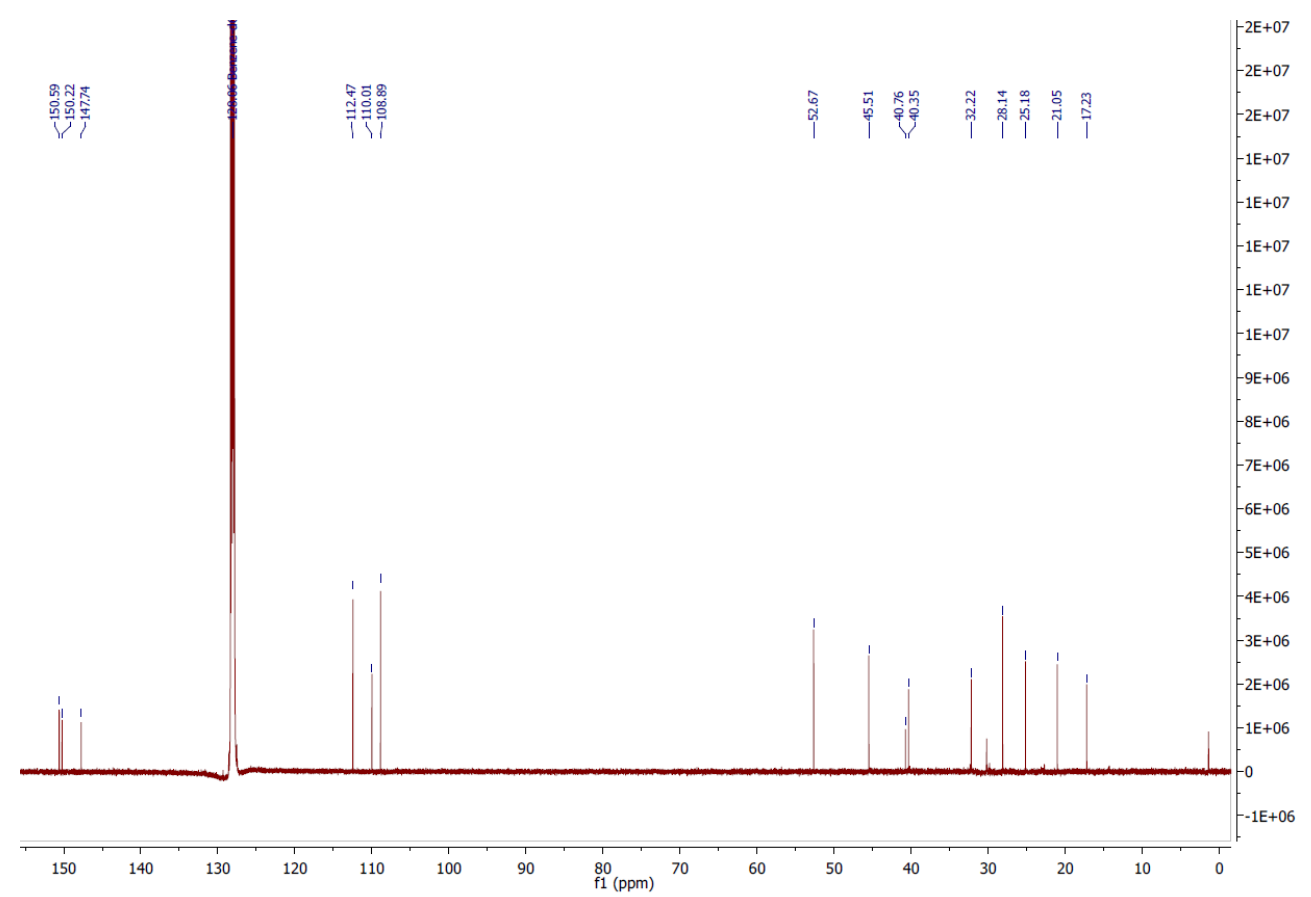


HSQC (31) - $600 \mathrm{MHz}, \mathrm{C}_{6} \mathrm{D}_{6}$

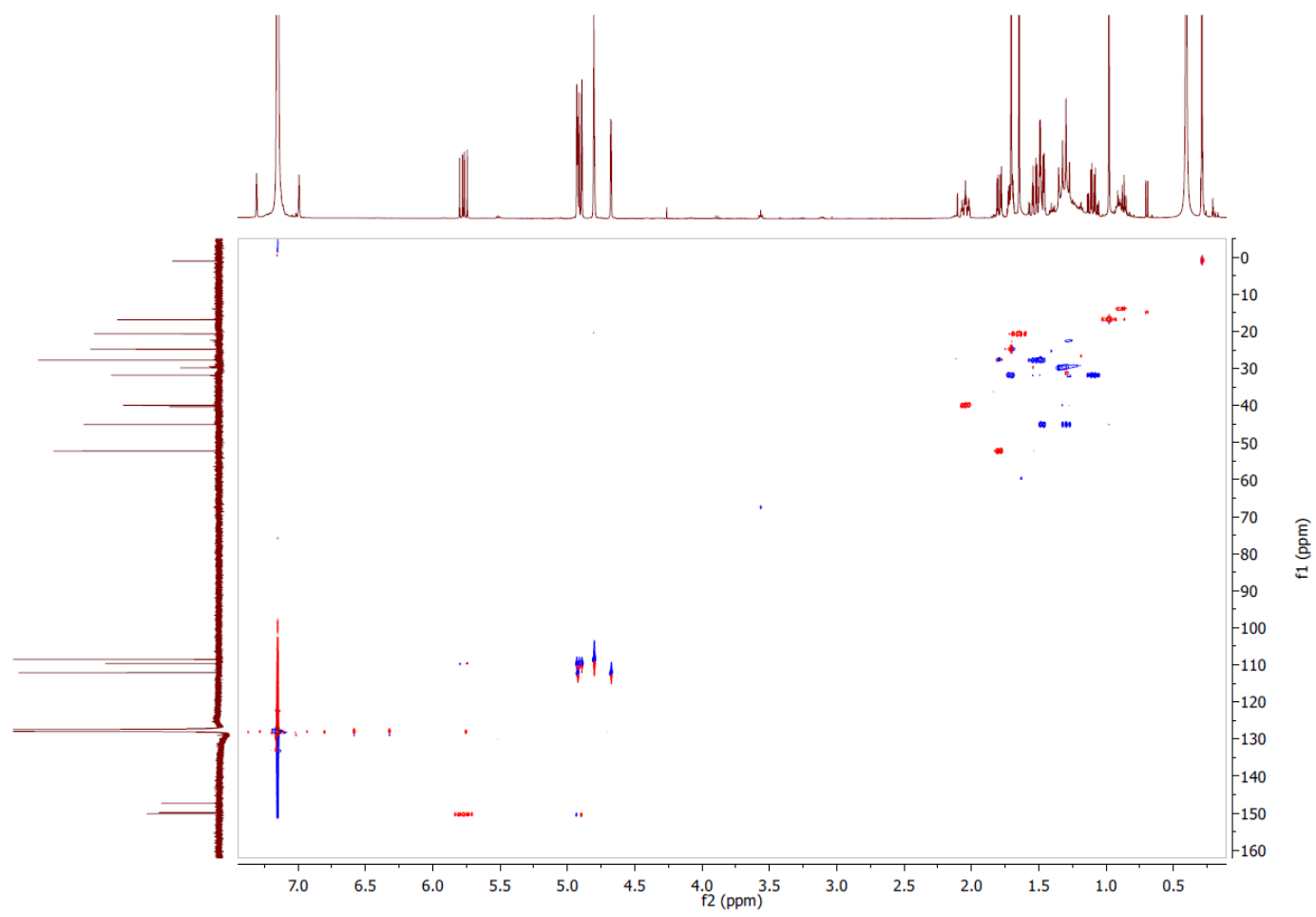

$\operatorname{COSY}(31)-600 \mathrm{MHz}, \mathrm{C}_{6} \mathrm{D}_{6}$

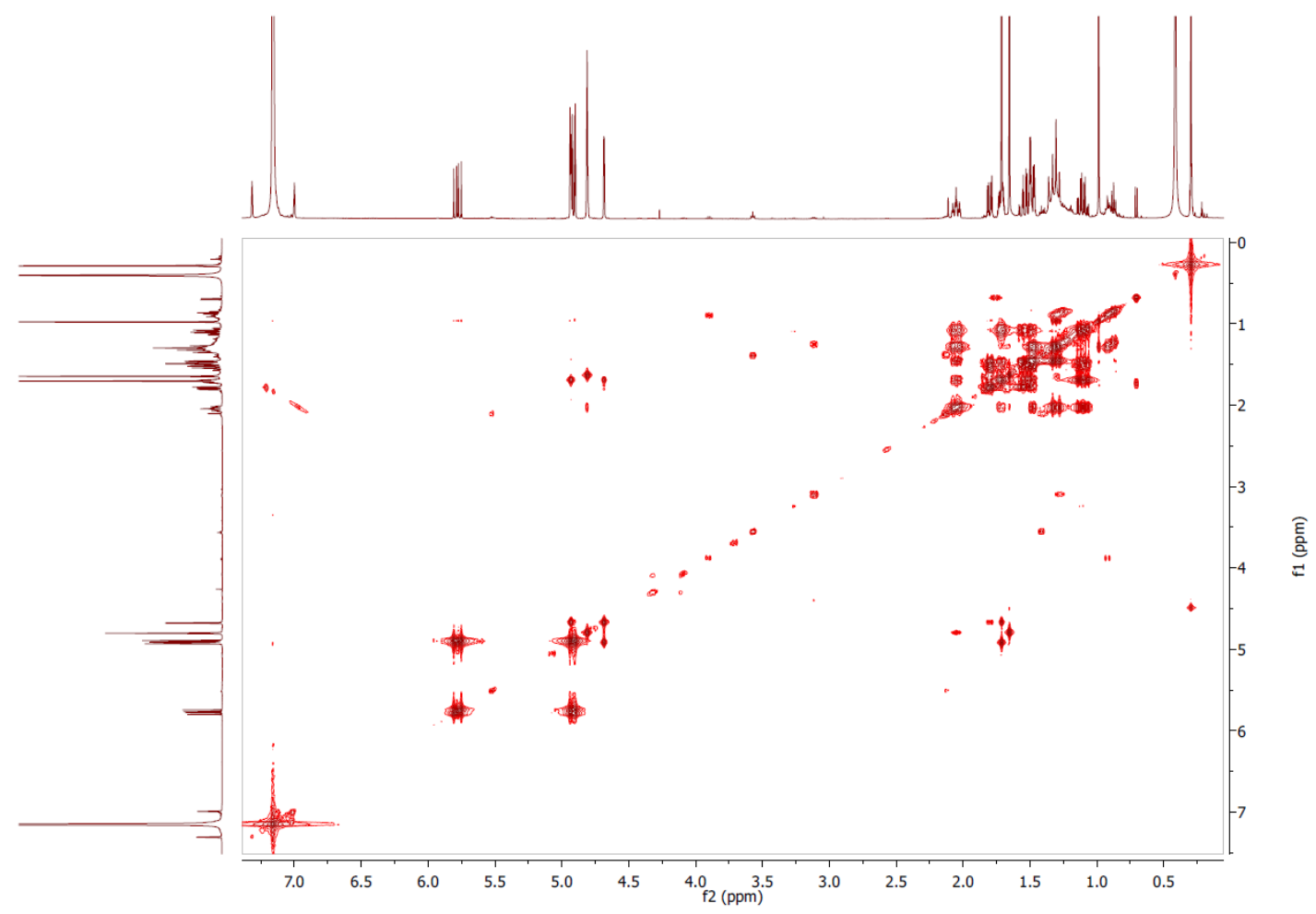


HMBC (31) - $600 \mathrm{MHz}, \mathrm{C}_{6} \mathrm{D}_{6}$

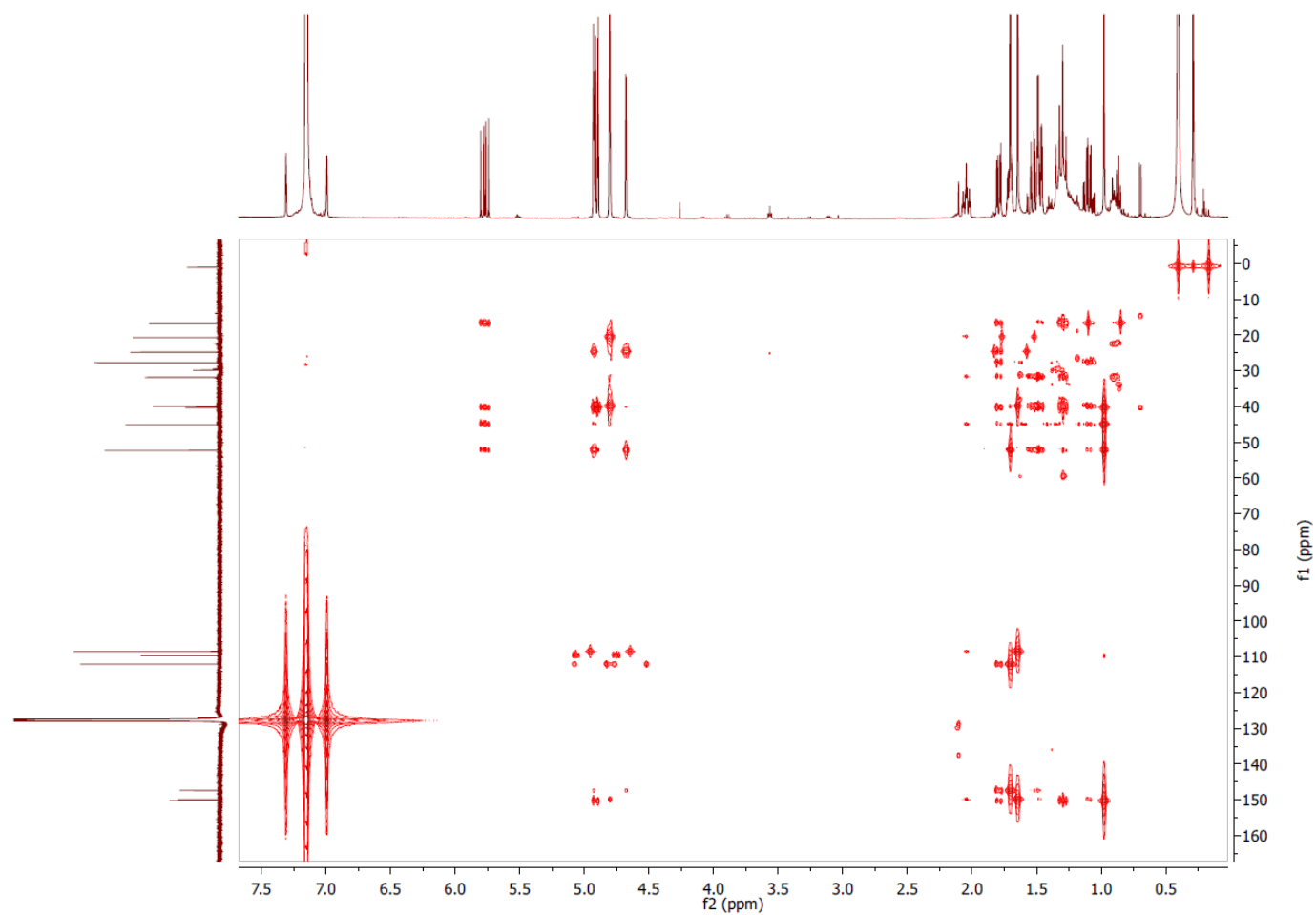

NOESY (31) - $600 \mathrm{MHz}, \mathrm{C}_{6} \mathrm{D}_{6}$

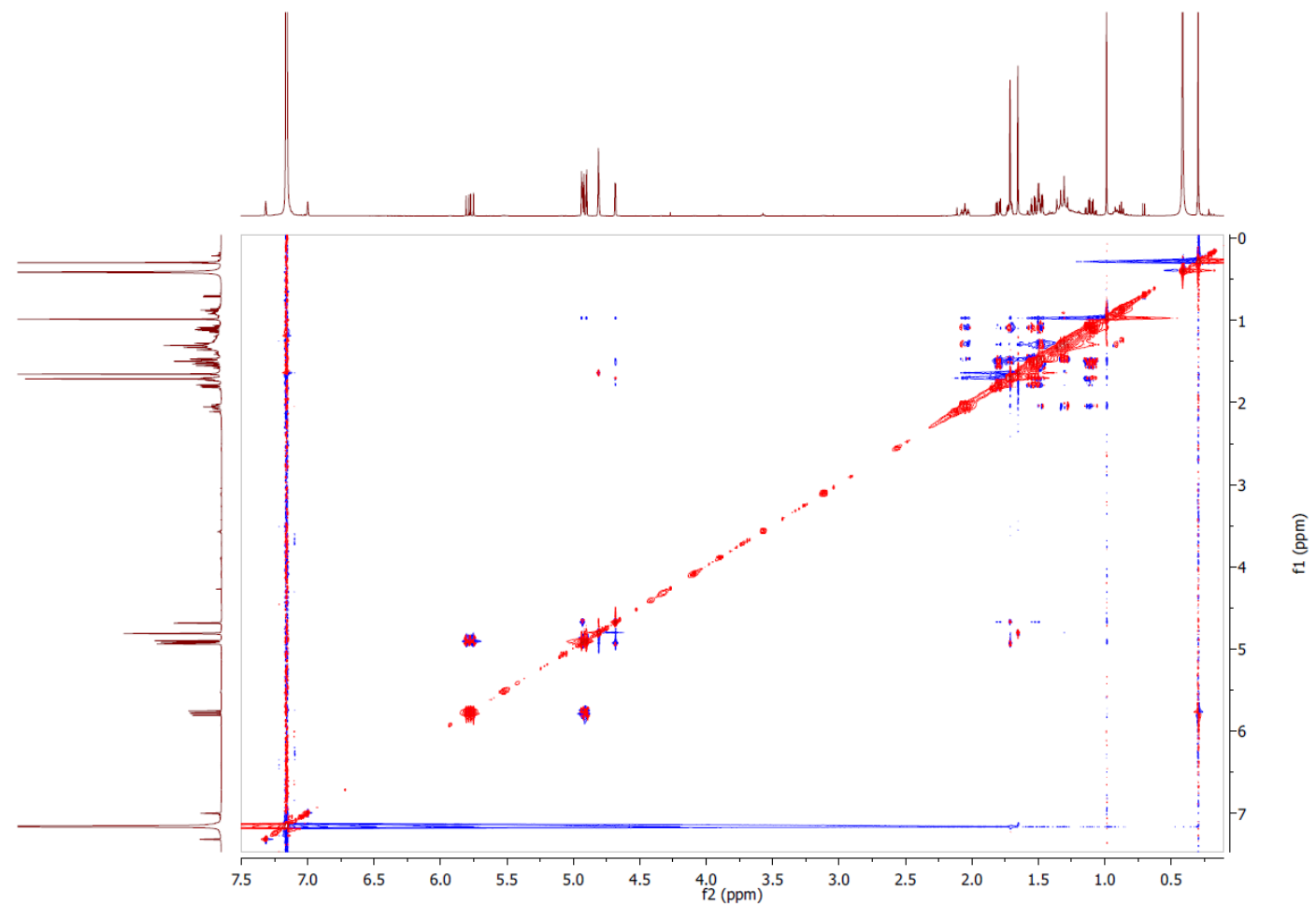




\section{References supporting information}

[S1] J. Leonard, B. Lygo, G. Procter, Praxis in der organischen Chemie, VCH, Weinheim, 1996 (ISBN 3-527-29411-2).

[S2] P. B. Shrestha-Dawadi, J. Lugtenburg, Eur. J. Org. Chem. 2003, 23, 4654-4663.

[S3] G. D. Coxon, B. L. Furman, A. L. Harvey, J. McTavish, M. H. Mooney, M. Arastoo, A. R. Kennedy, J. M. Tettey, R. D. Waigh, J. Med. Chem. 2009, 52, 3457- 3463.

[S4] C. M. Wang, R. Hopson, X. Lin, D. E. Cane, J. Am. Chem. Soc. 2003, 131, 8360-8361.

[S5] a) V. J. Davisson, A. B. Woodside, T. R. Neal, K. E. Stremler, M. Muehlbacher, C. D. Poulter, J. Org. Chem. 1986, 51, 4768-4779; b) A. B. Woodside, Z. Huang, C. D. Poulter, Org. Synth. 1988, 66, 211.

[S6] Y. Lee, E. M. Rochette, J. Kim, D. Y.-K. Chen, Angew. Chem. Int. Ed. 2017, 56, 12250.

[S7] K. M. Foote, M. John, G. Pattenden, Synlett 2001, 2001, 365.

[S8] G. Pattenden, S. J. Teague, J. Chem. Soc., Perkin Trans. 1 1988, 1077.

[S9] J. C. Cochran, V. Prindle, H. A. Young, M. H. Kumar, S. Tom, N. D. K. Petraco, C. Mohoro, B. Kelley, Synth. React. Inorg. Meth.-Org. Chem. 2002, 32, 885.

[S10] C. Cook, X. Guinchard, F. Liron, E. Roulland, Org. Lett. 2010, 12, 744.

[S11] E.-i. Negishi, S.-Y. Liou, C. Xu, S. Huo, Org. Lett. 2002, 4, 261.

[S12] F. G. Bordwell, P. S. Landis, J. Am. Chem. Soc. 1957, 79, 1593.

[S13] E. Negishi, G. Wang, Science of Synthesis, 2010, 47, 979.

[S14] M. Jegelka, B. Plietker, Org. Lett. 2009, 11, 3462-3465.

[S15] S. Combemale, J. Assam-Evoung, S. Houaidji, R. Bibi, V. Barragan-Montero, Molecules 2014, $19,1120-1149$. 


\section{Attachments}

\section{A. GC chromatograms and MS spectra}

Product 25
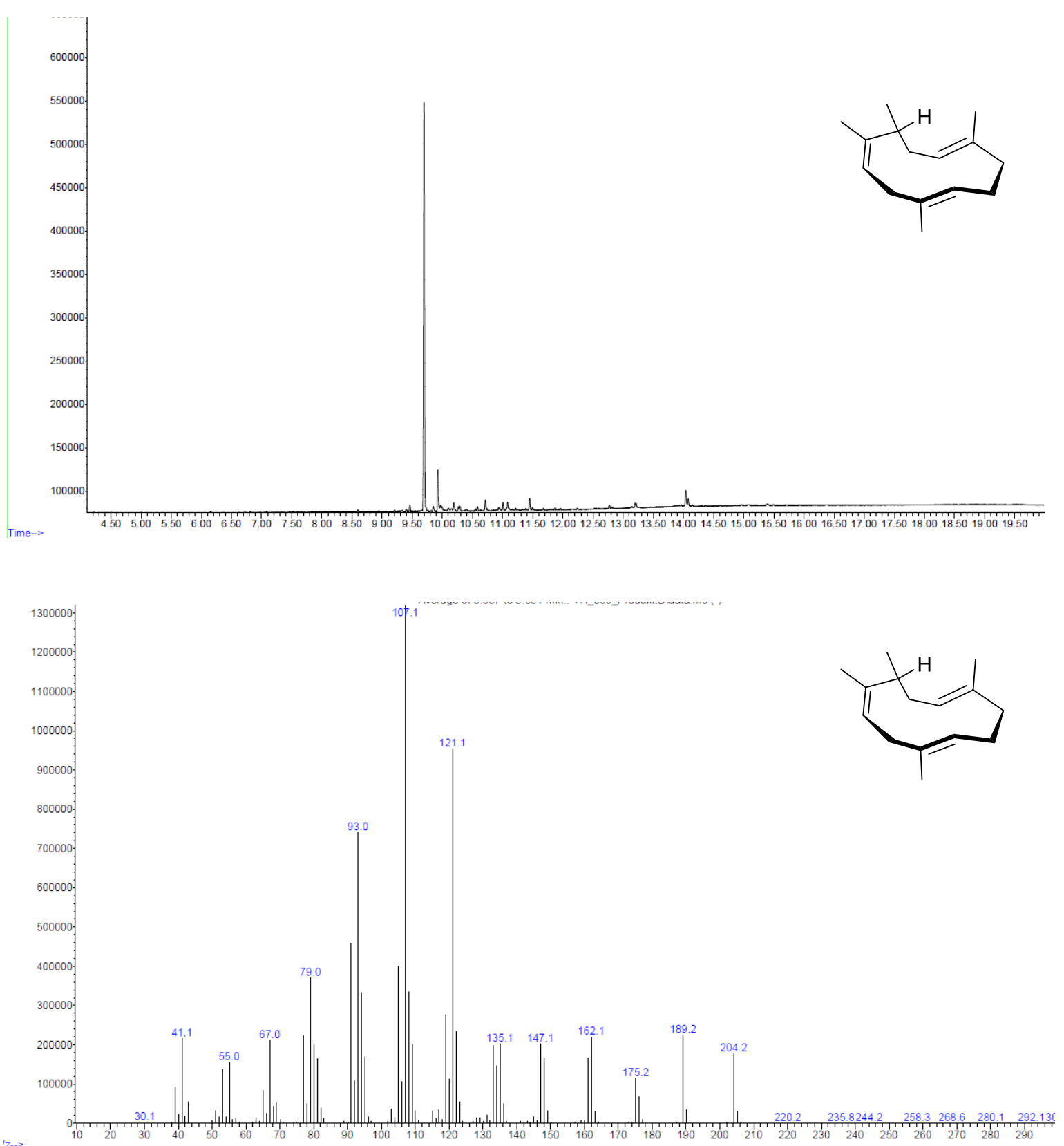
Product 26
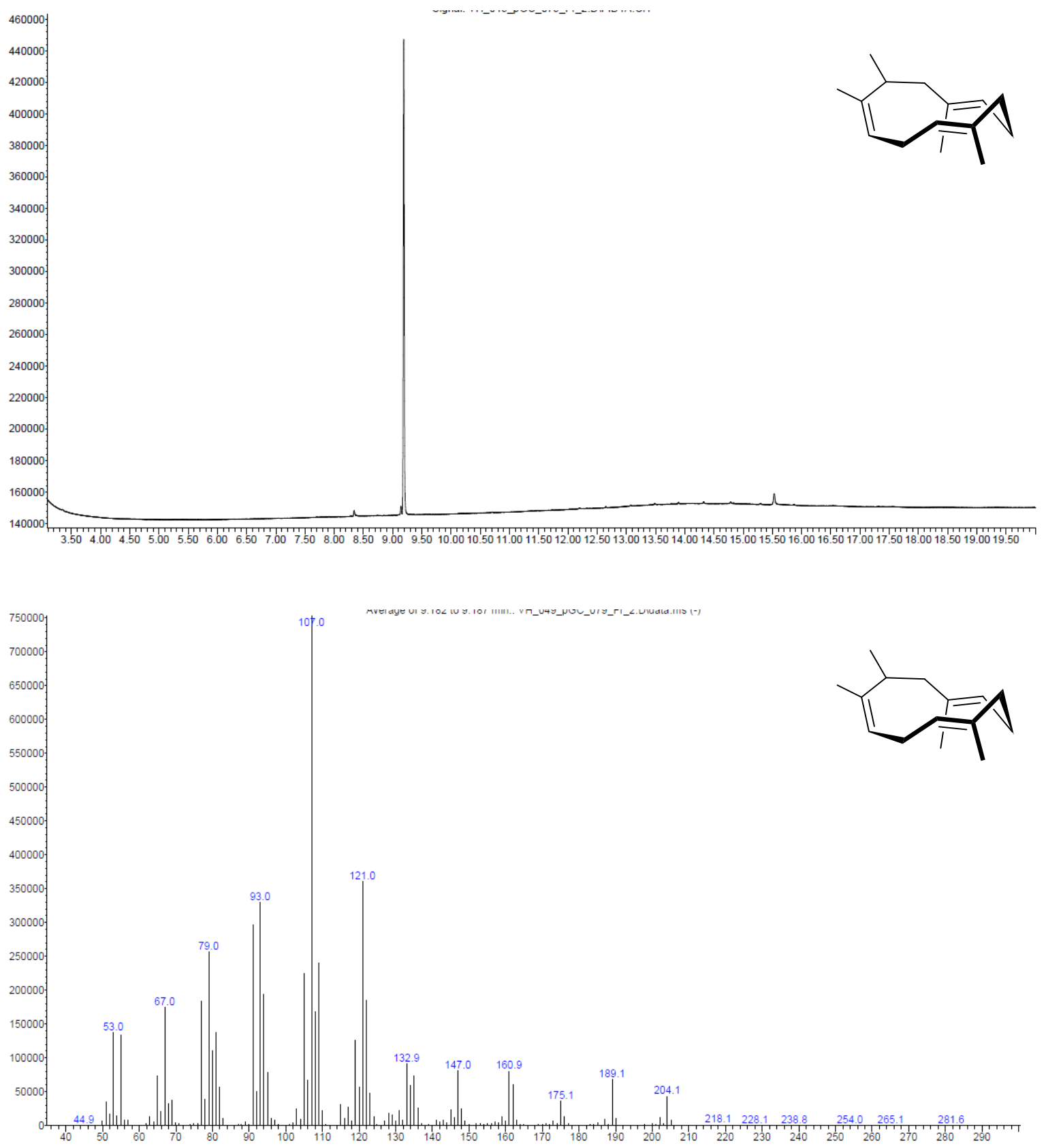


\section{Product 27}
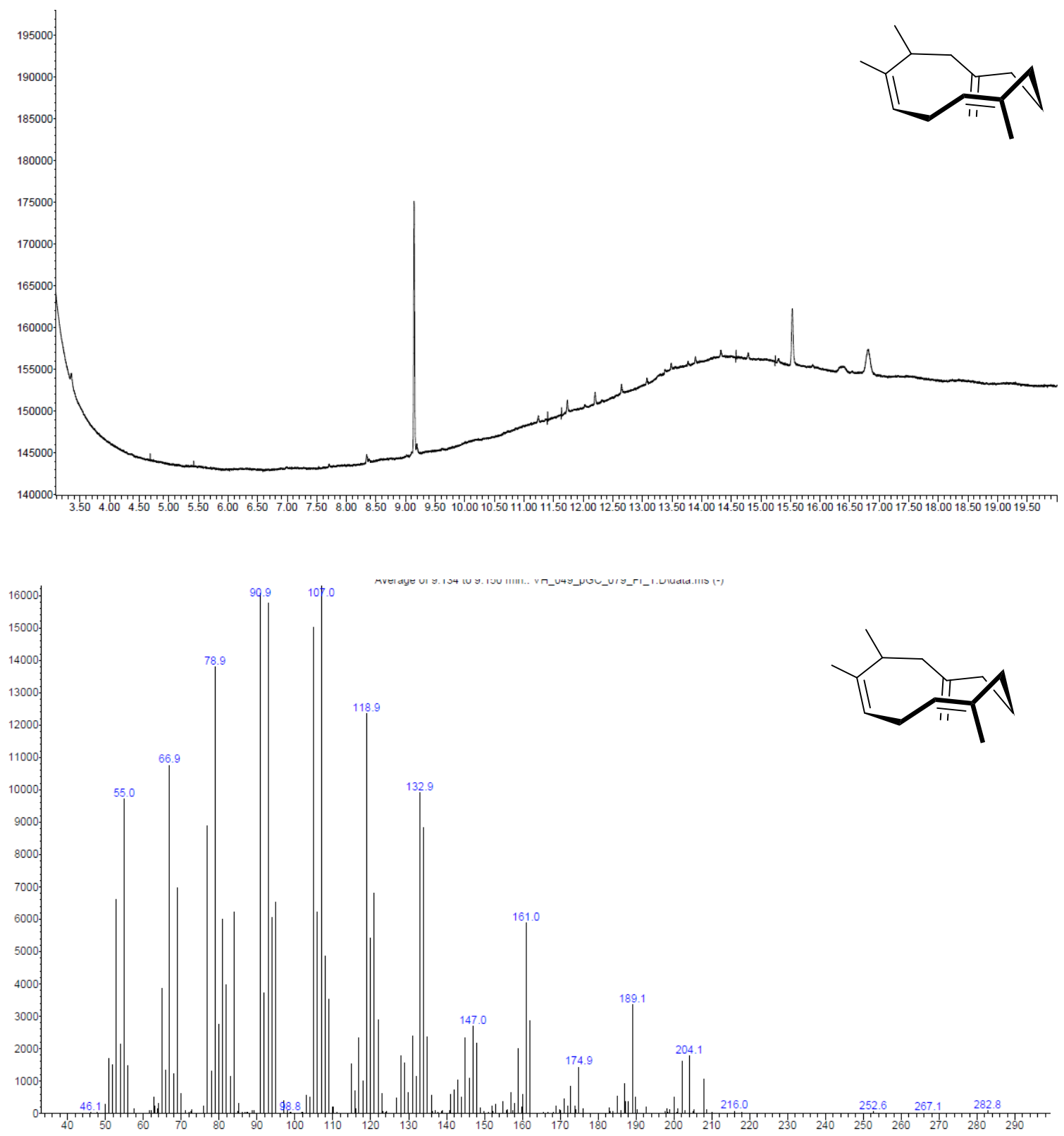
Product 28
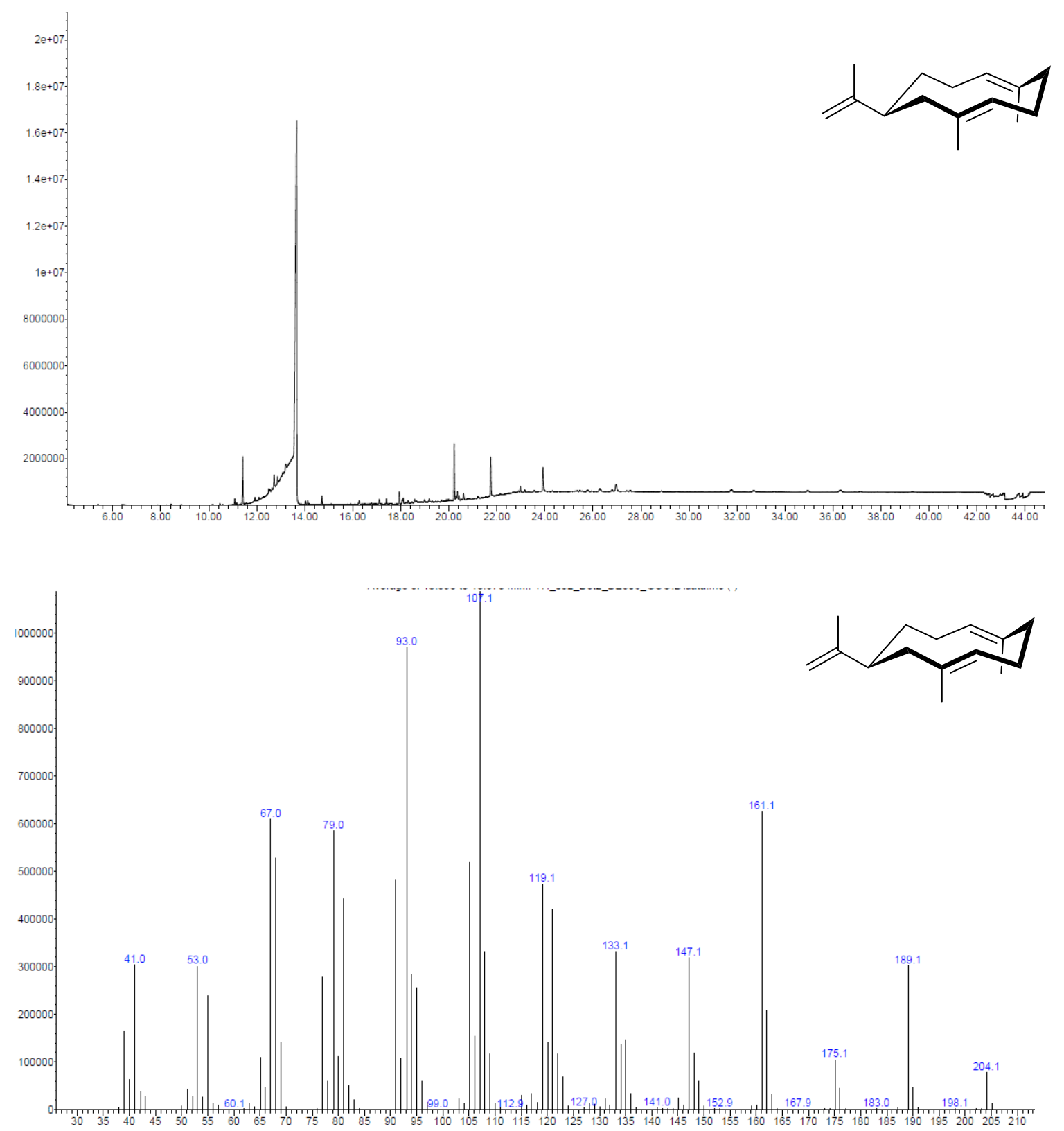


\section{Product 31}
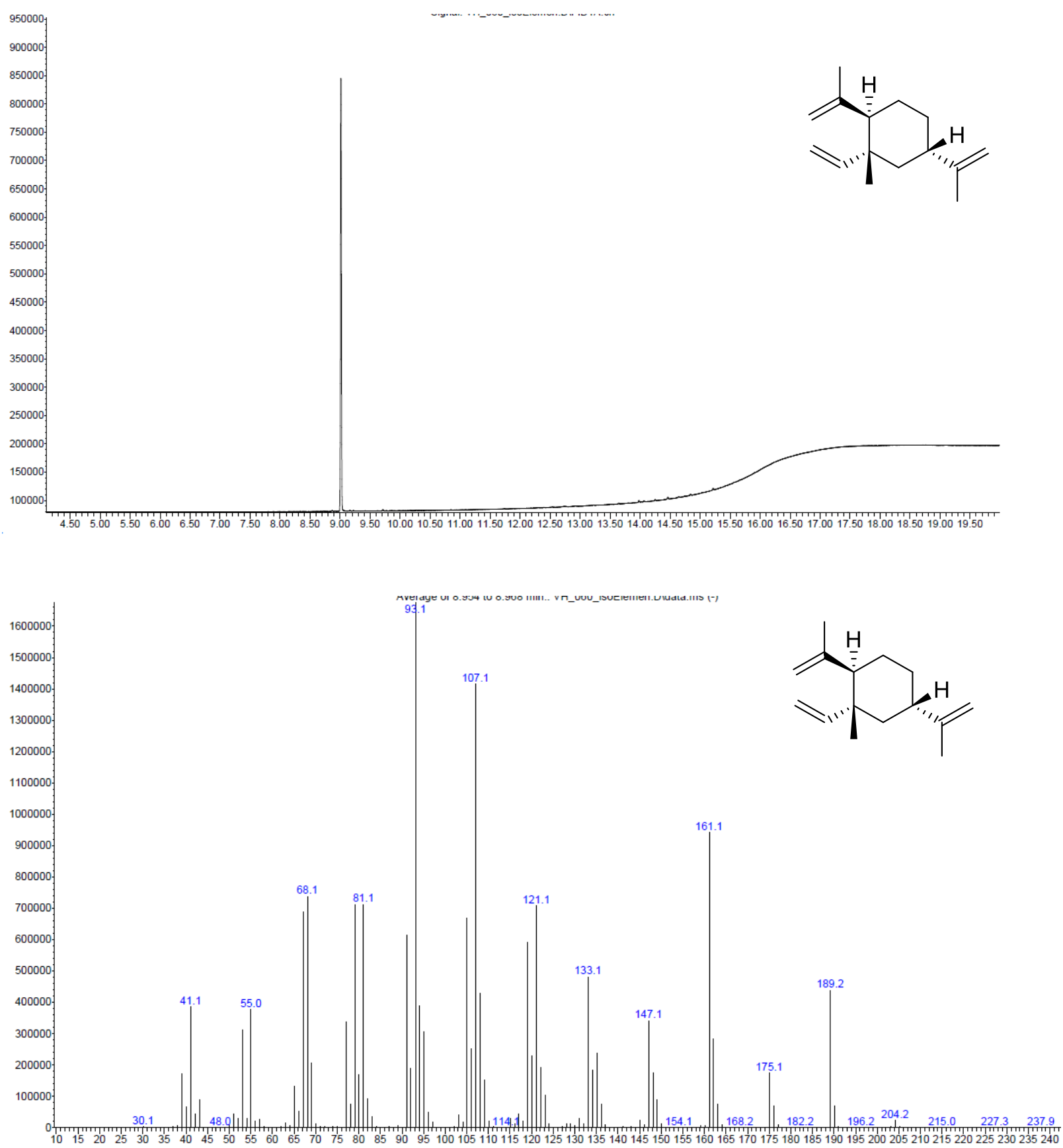
B. Copies of ${ }^{1} \mathrm{H}$ - and ${ }^{13} \mathrm{C}$-NMR spectra (chemical synthesis)

${ }^{1} \mathrm{H}-\mathrm{NMR}\left(400 \mathrm{MHz}, \mathrm{CDCl}_{3}\right)$ :
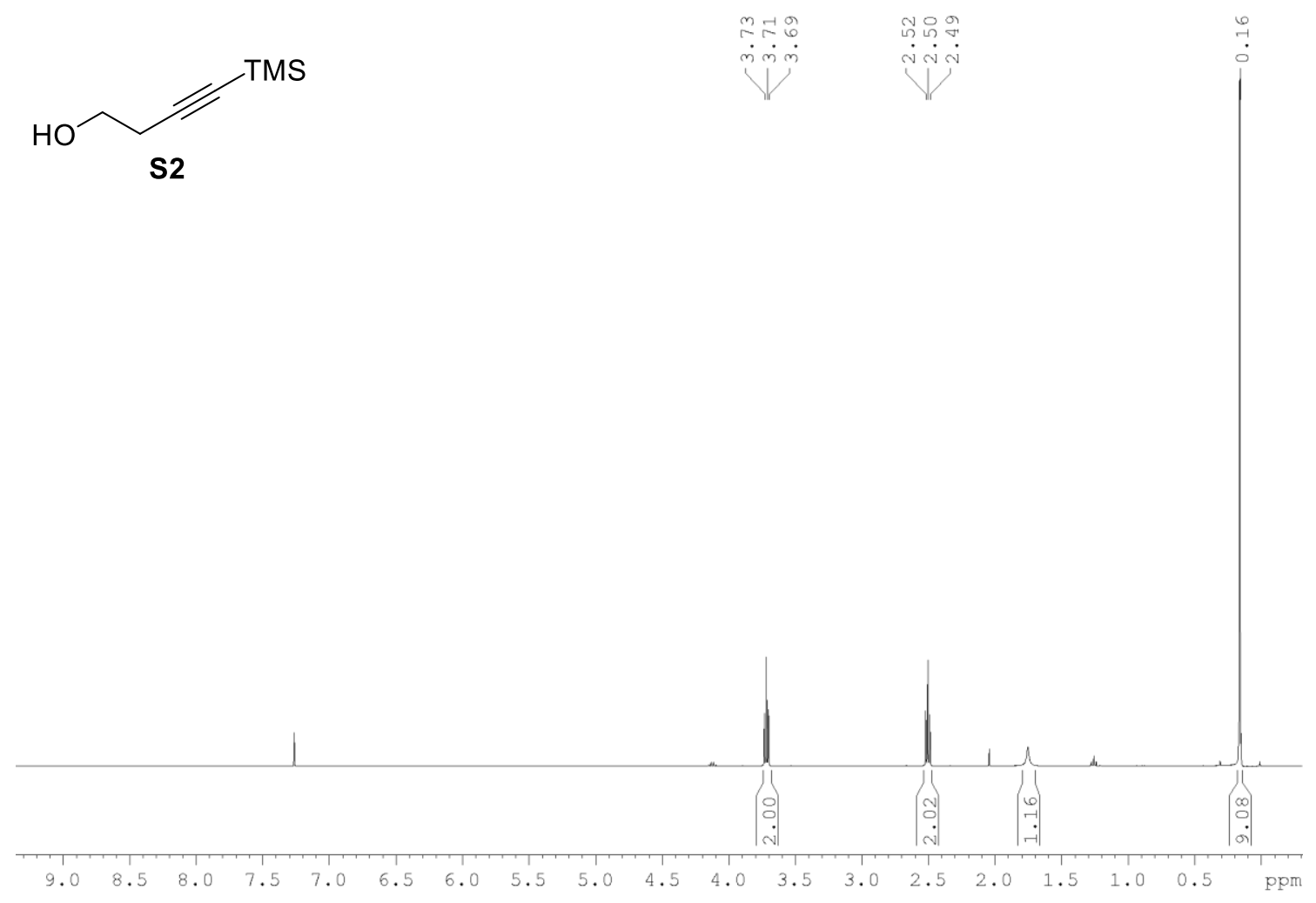

${ }^{13} \mathrm{C}-\mathrm{NMR}\left(100 \mathrm{MHz}, \mathrm{CDCl}_{3}\right)$ :
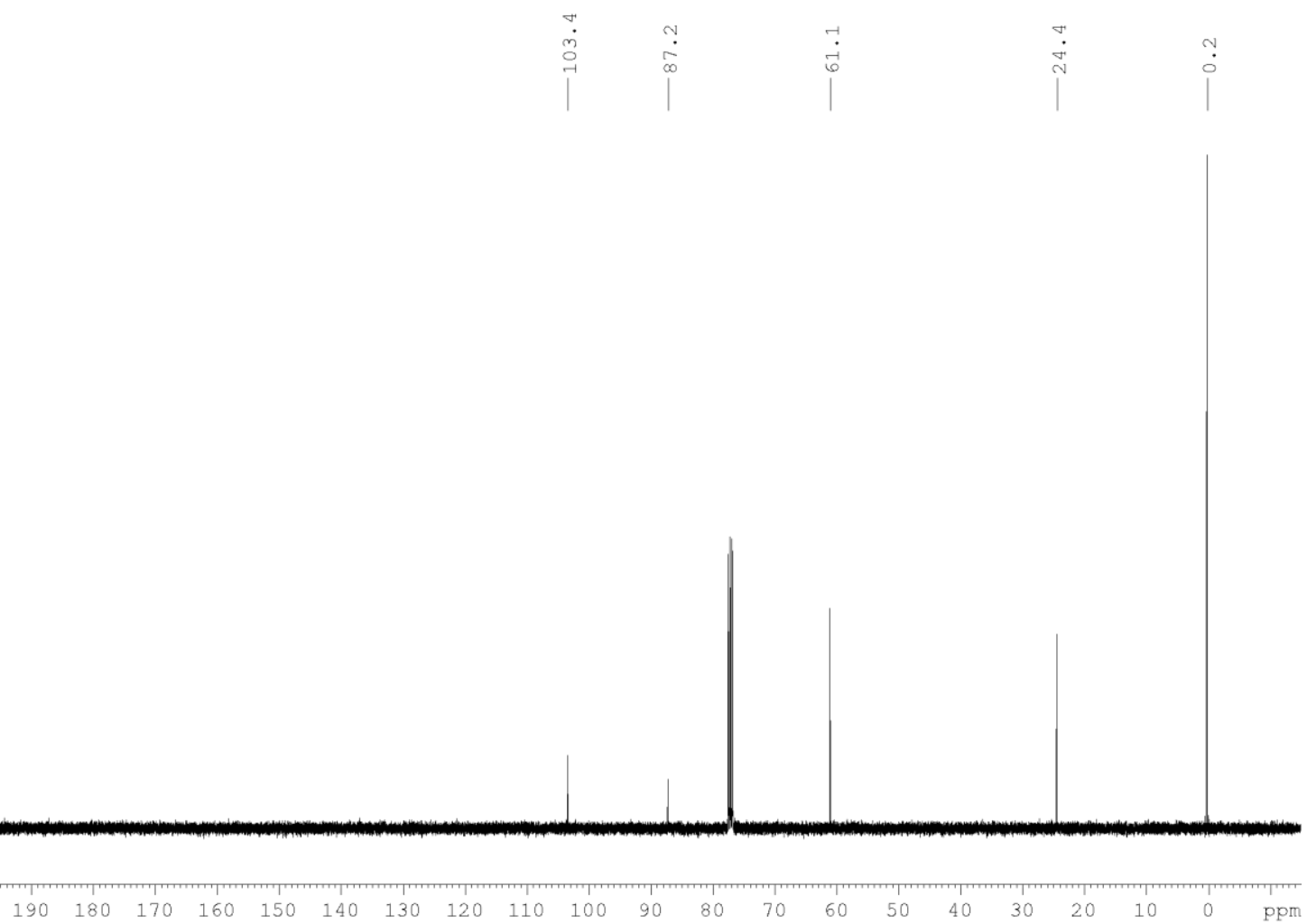
${ }^{1} \mathrm{H}-\mathrm{NMR}\left(400 \mathrm{MHz}, \mathrm{CDCl}_{3}\right)$ :

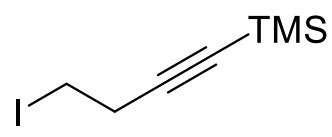

14

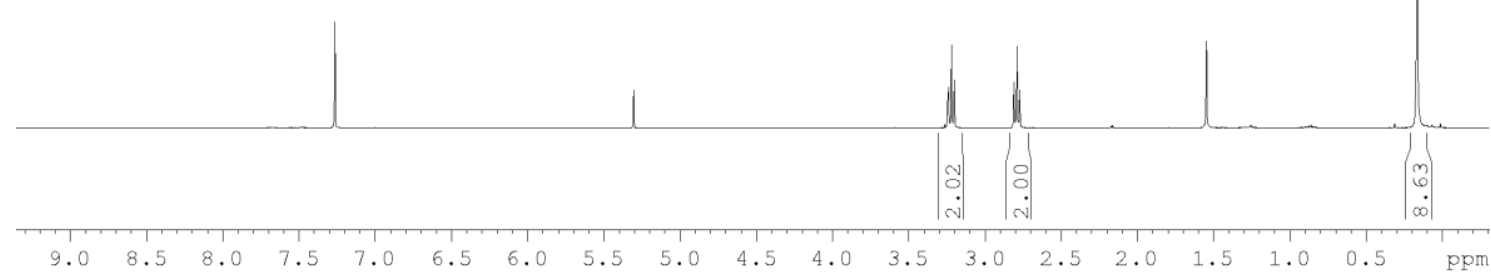

${ }^{13} \mathrm{C}-\mathrm{NMR}\left(100 \mathrm{MHz}, \mathrm{CDCl}_{3}\right)$ :

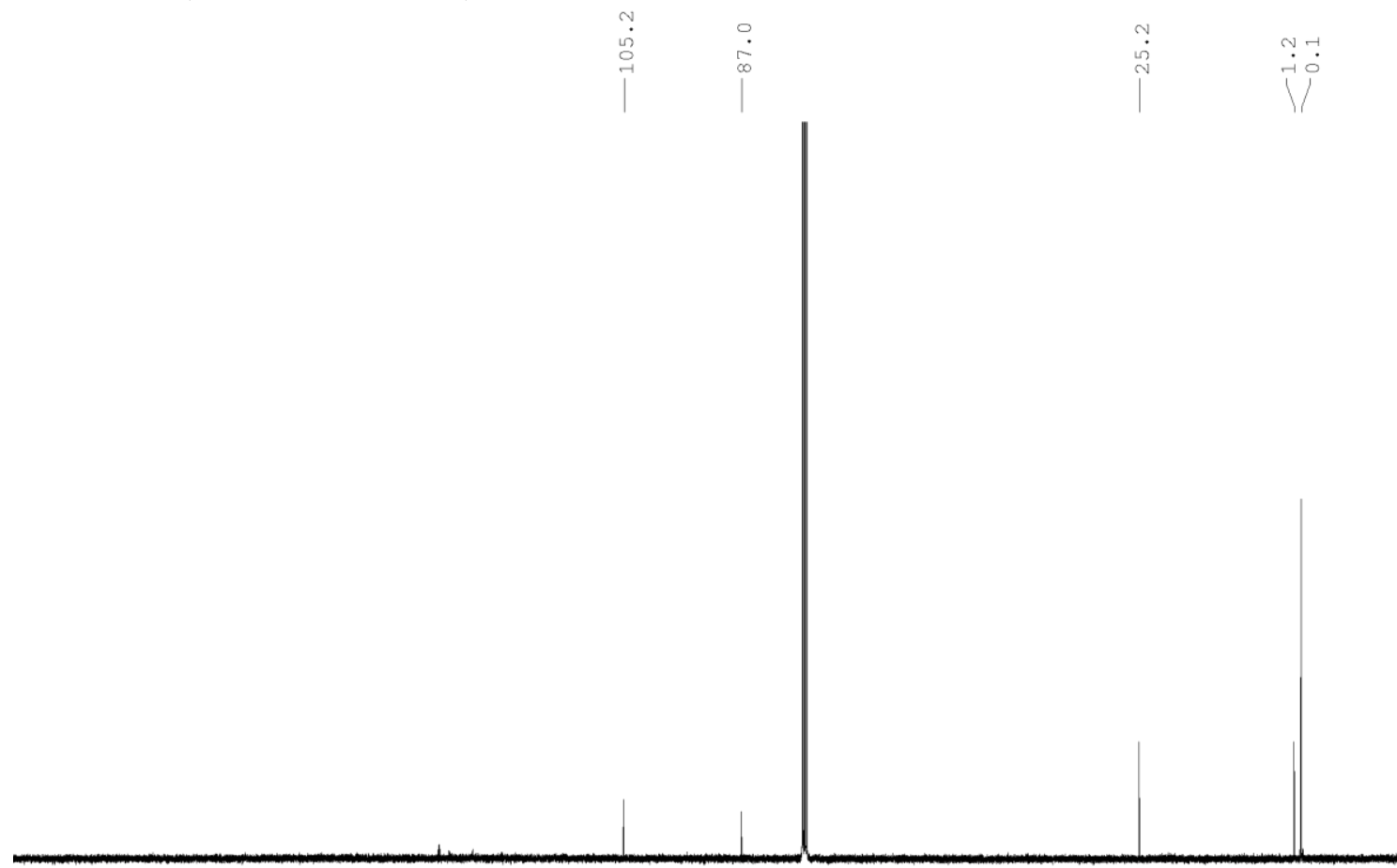


${ }^{1} \mathrm{H}-\mathrm{NMR}\left(400 \mathrm{MHz}, \mathrm{CDCl}_{3}\right)$ :<smiles>C/C(=C\I)CCO</smiles>

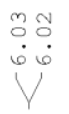

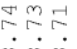

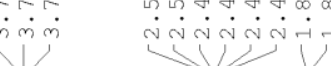

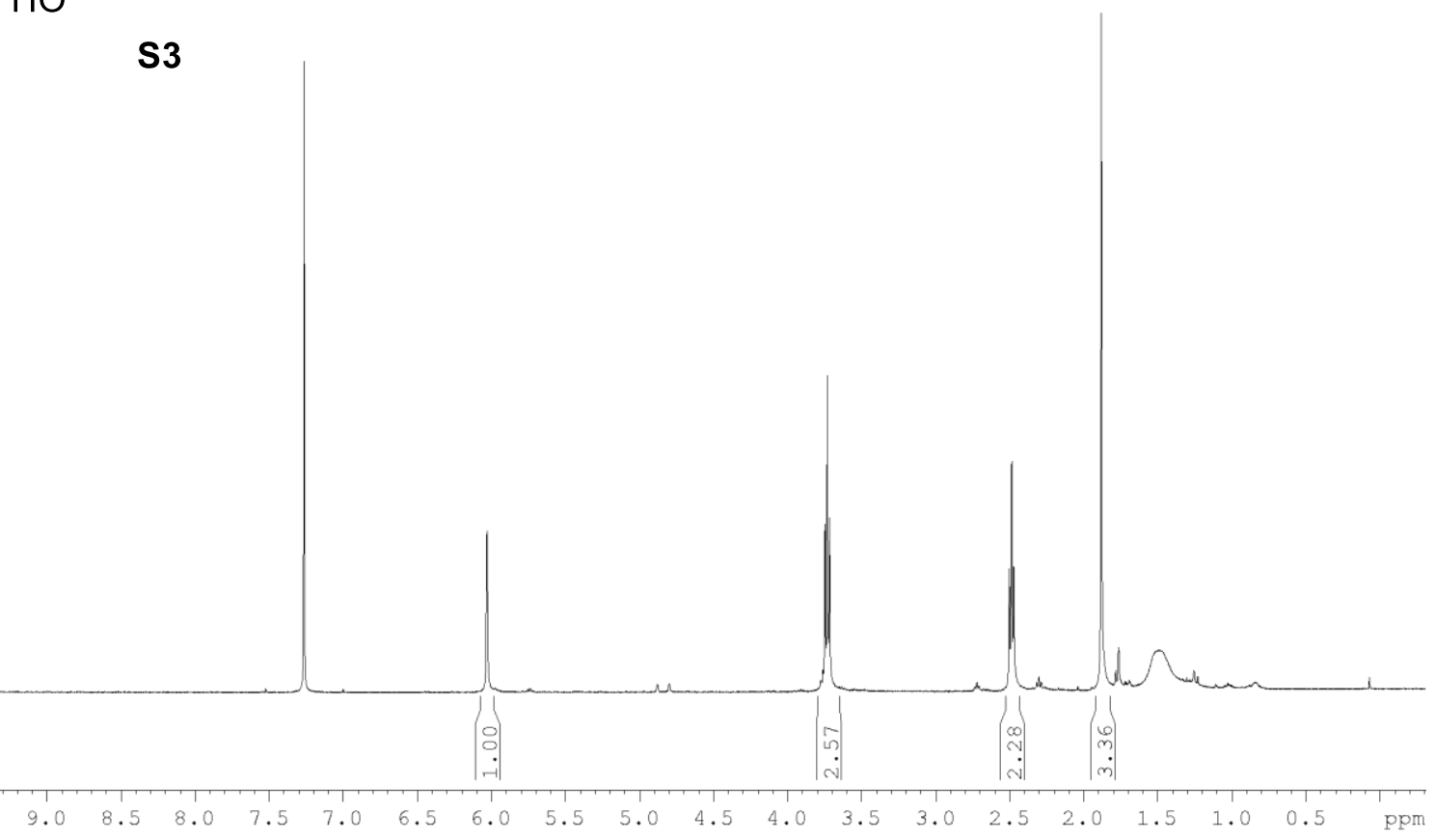

${ }^{13} \mathrm{C}-\mathrm{NMR}\left(100 \mathrm{MHz}, \mathrm{CDCl}_{3}\right)$ :

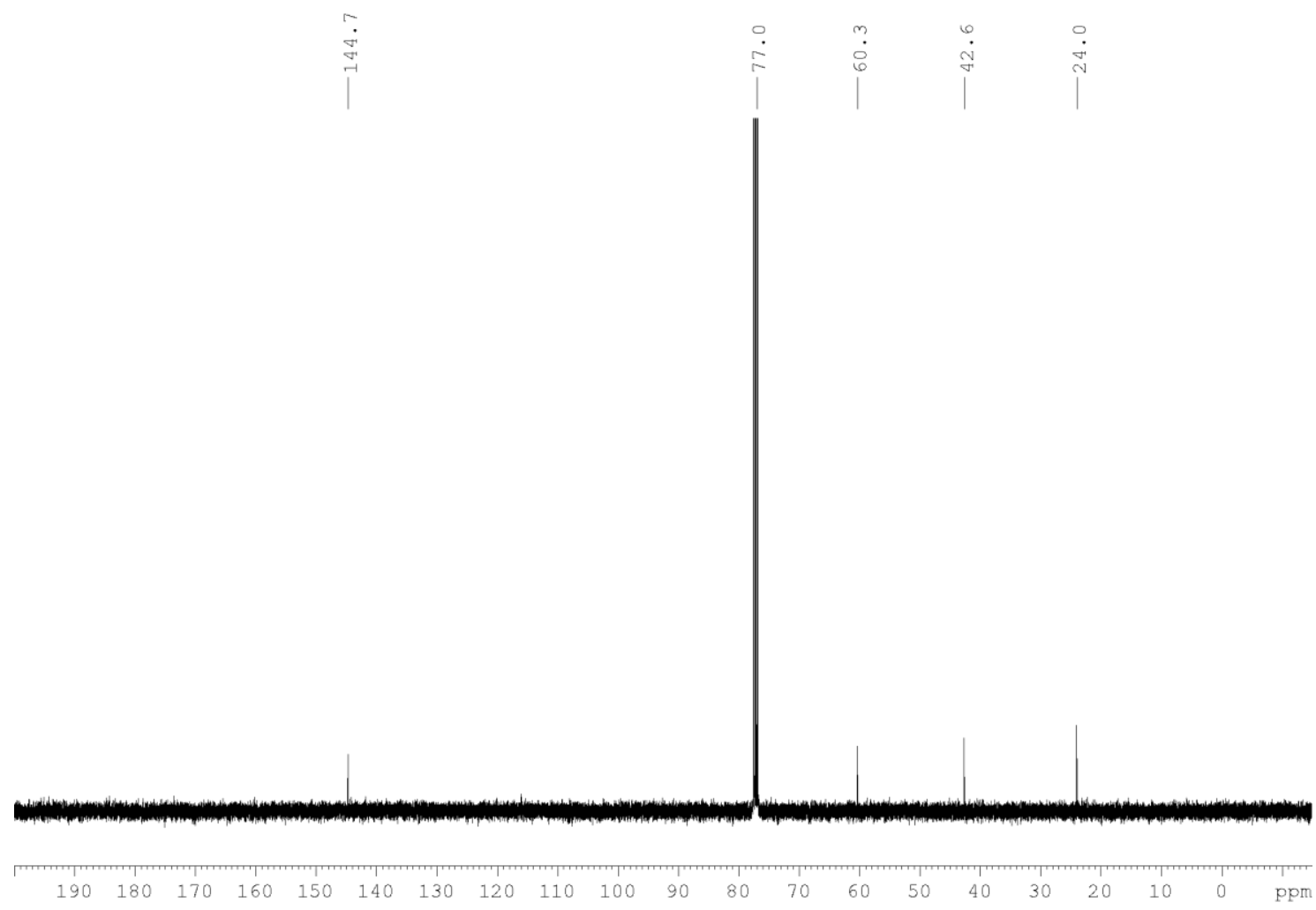


${ }^{1} \mathrm{H}-\mathrm{NMR}\left(400 \mathrm{MHz}, \mathrm{CDCl}_{3}\right)$ :<smiles>C/C(=C\I)CCI</smiles>

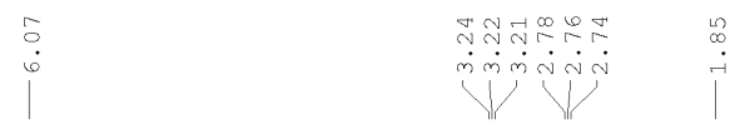

15

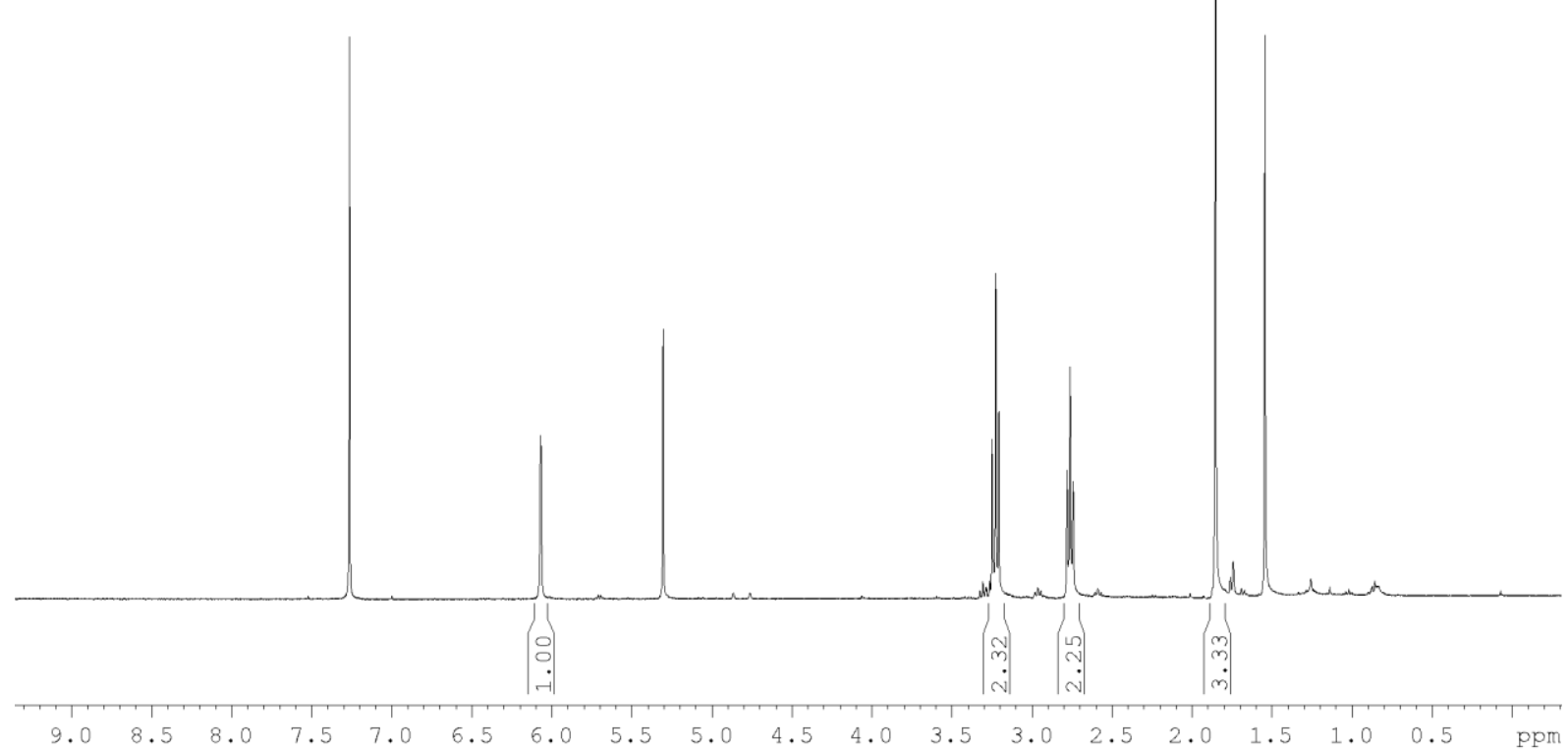

${ }^{13} \mathrm{C}-\mathrm{NMR}\left(100 \mathrm{MHz}, \mathrm{CDCl}_{3}\right)$ :

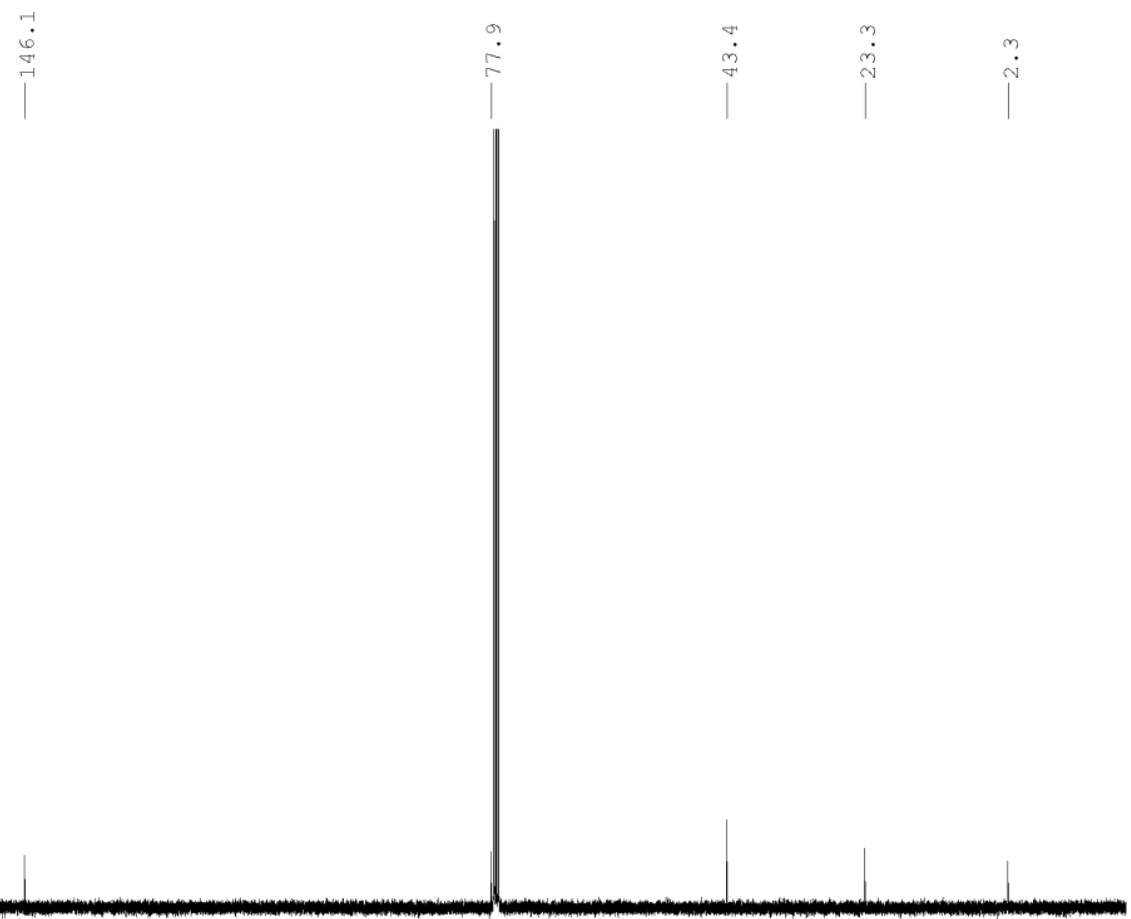

$\begin{array}{llllllllllllllllllll}190 & 180 & 170 & 160 & 150 & 140 & 130 & 120 & 110 & 100 & 90 & 80 & 70 & 60 & 50 & 40 & 30 & 20 & 10 & 0\end{array}$ 
${ }^{1} \mathrm{H}-\mathrm{NMR}\left(400 \mathrm{MHz}, \mathrm{CDCl}_{3}\right)$ :

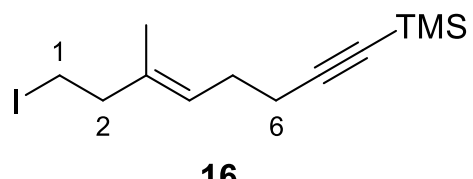

16

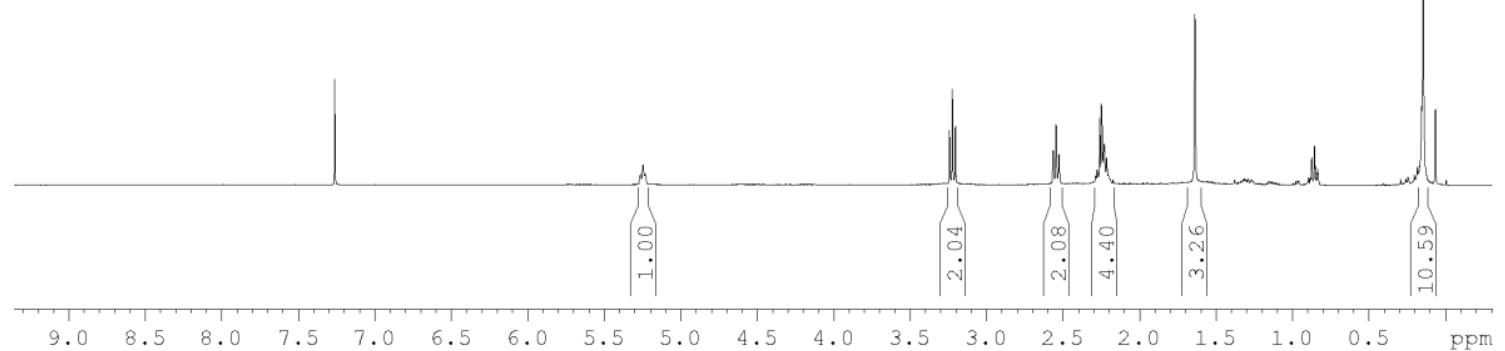

${ }^{13} \mathrm{C}-\mathrm{NMR}\left(100 \mathrm{MHz}, \mathrm{CDCl}_{3}\right)$ :
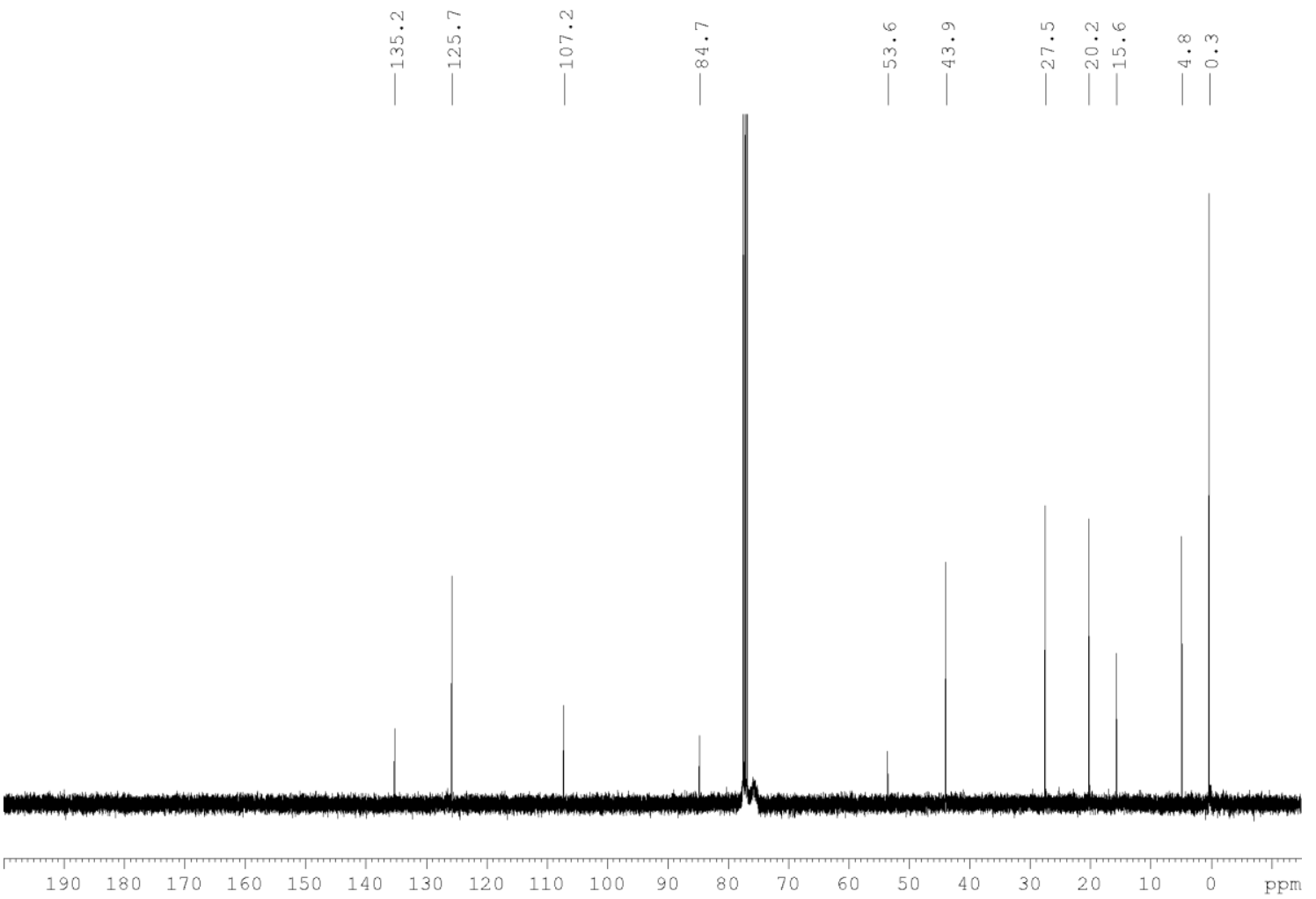
${ }^{1} \mathrm{H}-\mathrm{NMR}\left(400 \mathrm{MHz}, \mathrm{CDCl}_{3}\right.$ ):<smiles>CC=C(C)Br</smiles>

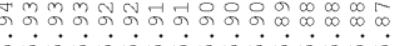

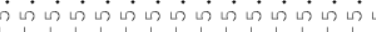

17
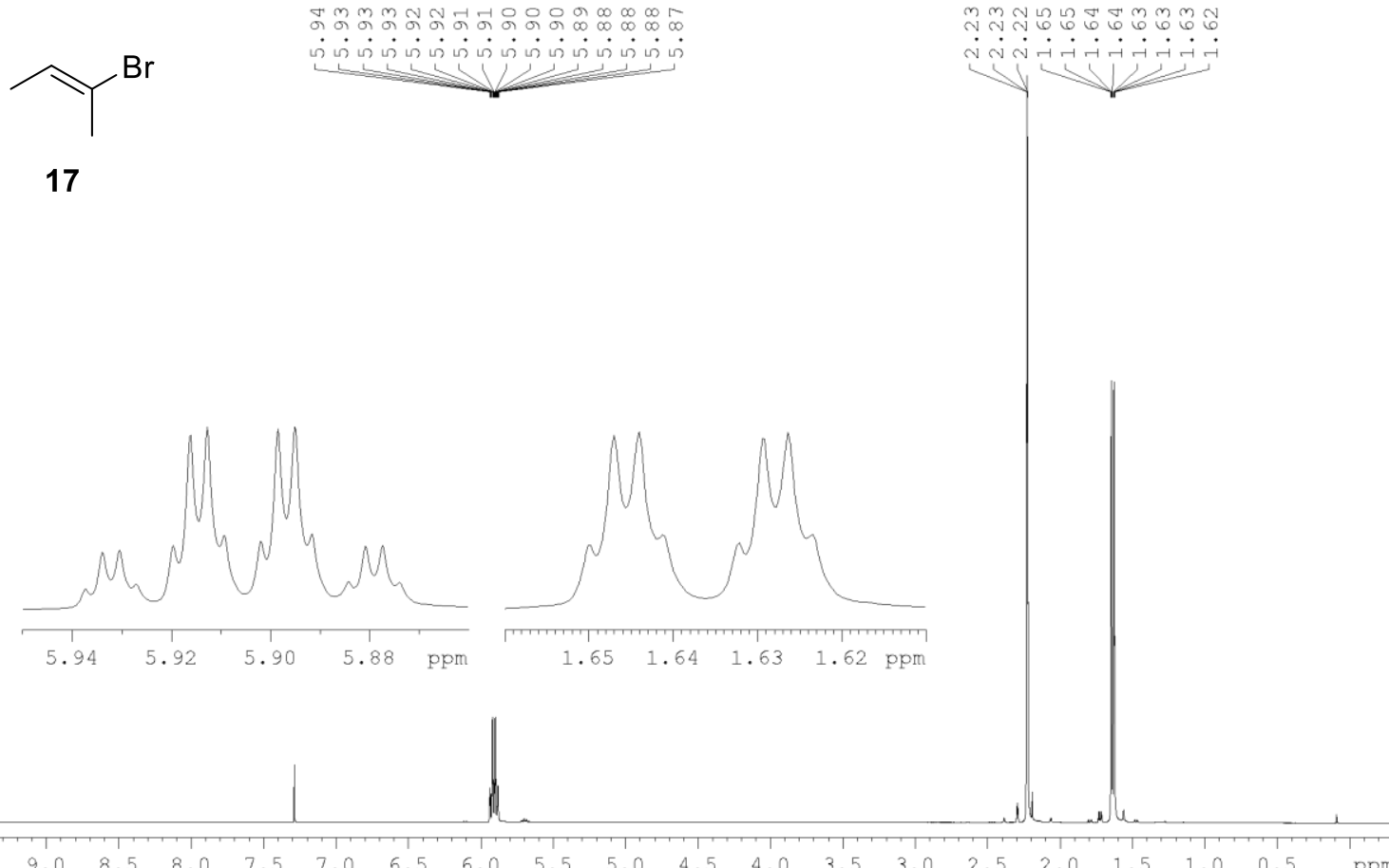

${ }^{13} \mathrm{C}-\mathrm{NMR}\left(100 \mathrm{MHz}, \mathrm{CDCl}_{3}\right)$ :
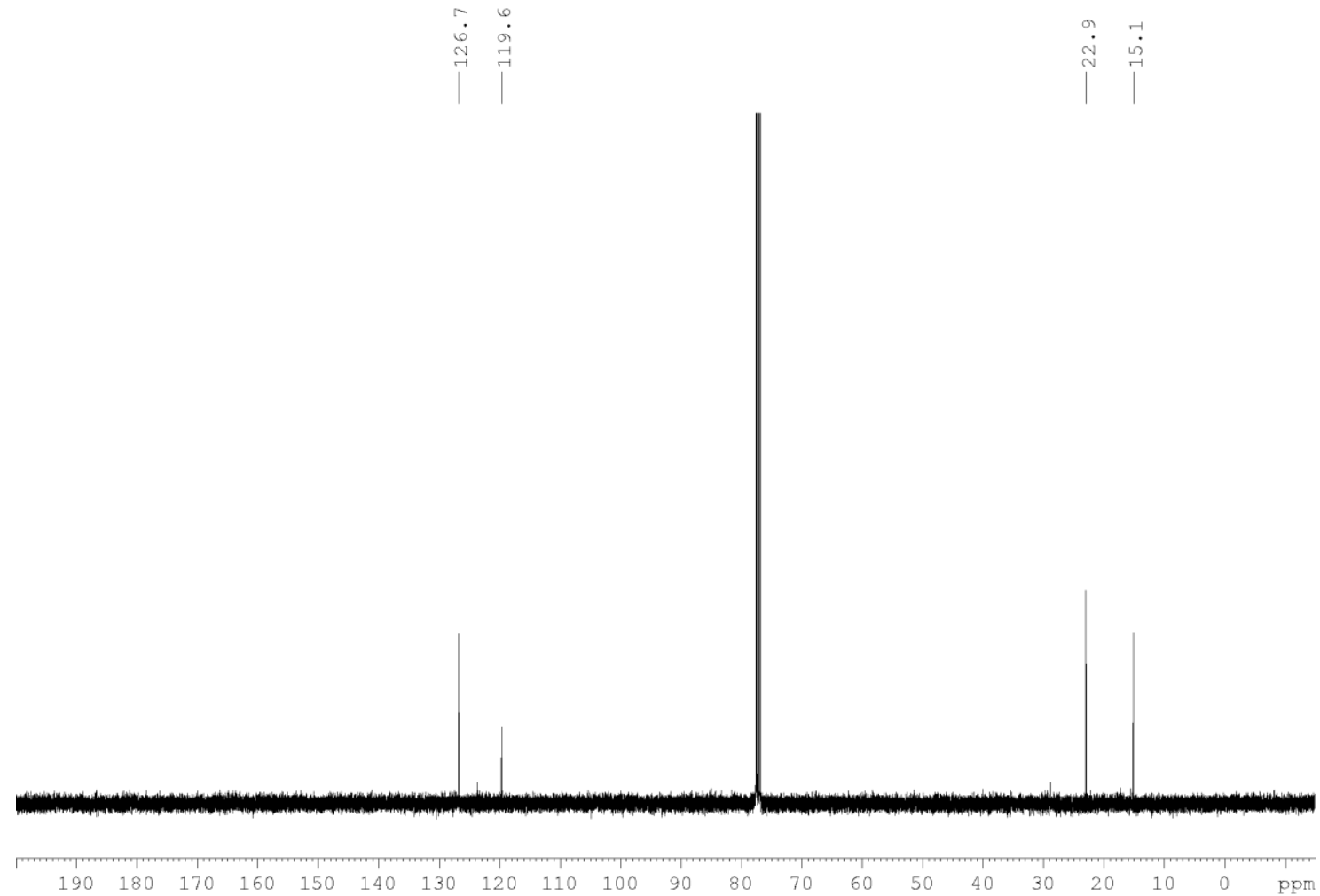
${ }^{1} \mathrm{H}-\mathrm{NMR}\left(400 \mathrm{MHz}, \mathrm{CDCl}_{3}\right.$ ):

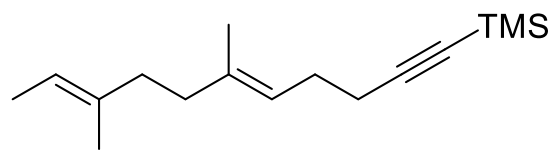

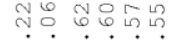

ำำ

ij $\dot{j} \dot{j}$
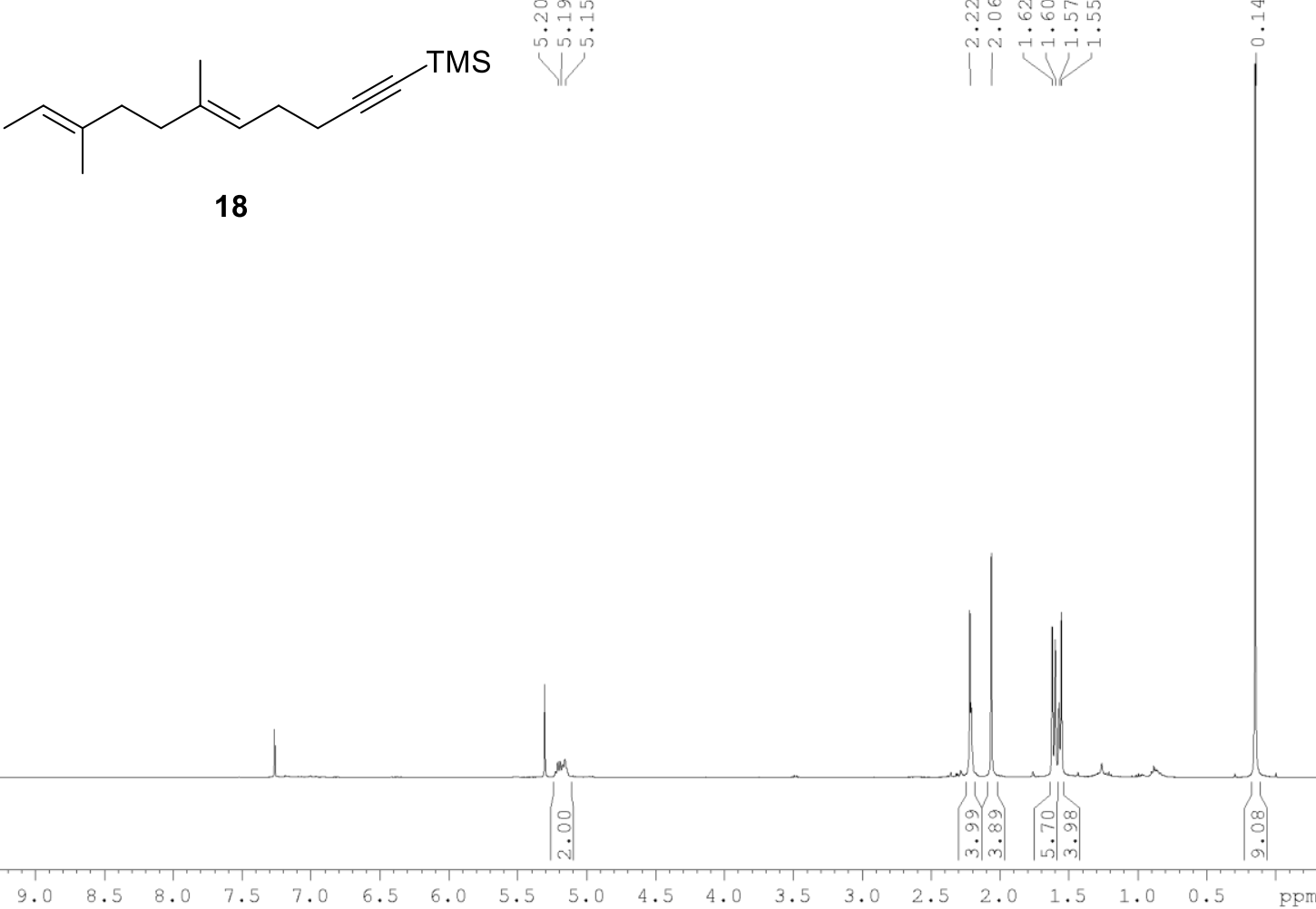

${ }^{13} \mathrm{C}-\mathrm{NMR}\left(100 \mathrm{MHz}, \mathrm{CDCl}_{3}\right)$ :
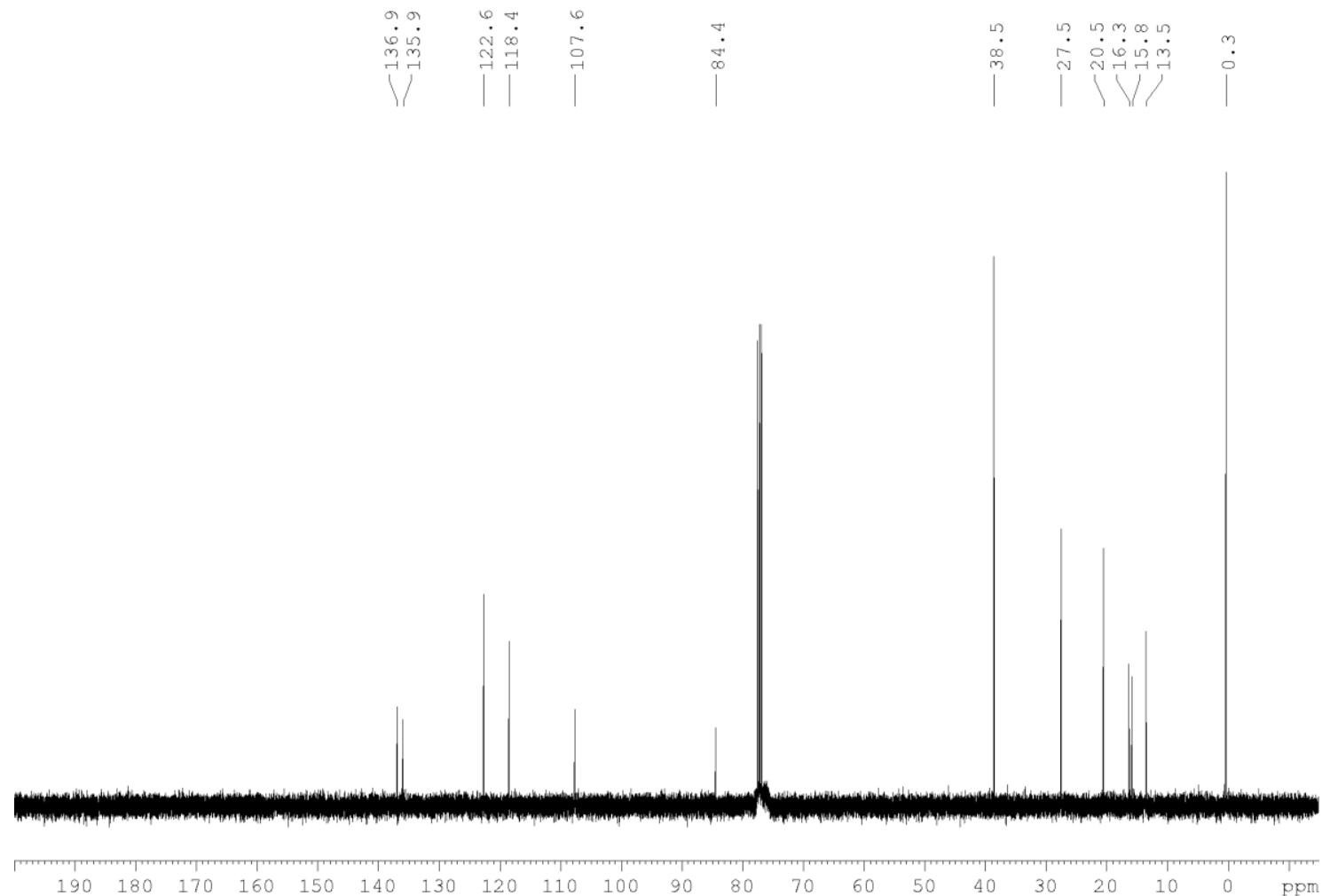
${ }^{1} \mathrm{H}-\mathrm{NMR}\left(400 \mathrm{MHz}, \mathrm{CDCl}_{3}\right.$ ):

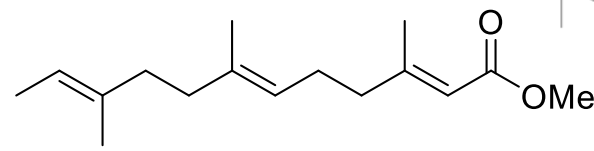

S5

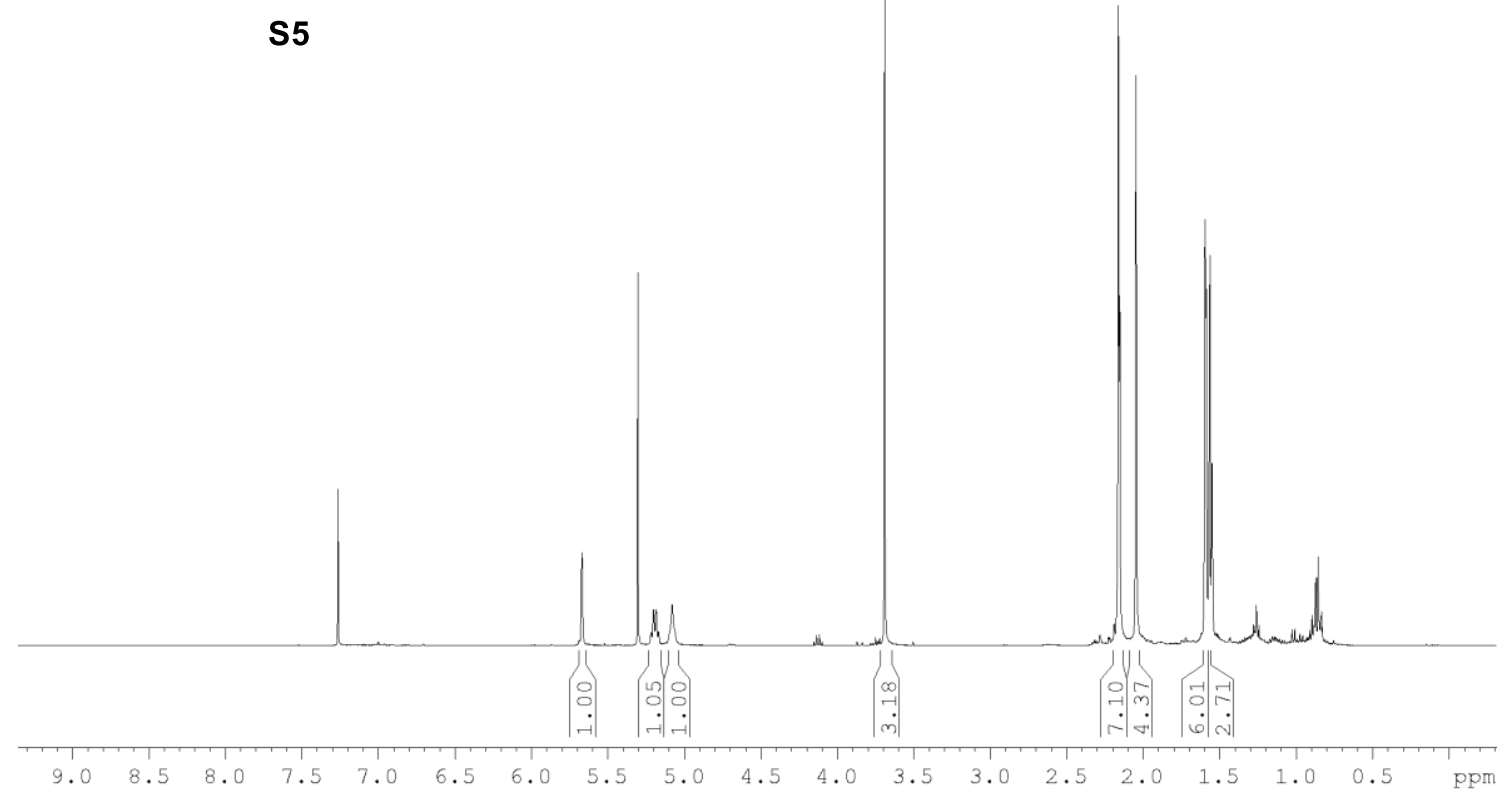

${ }^{13} \mathrm{C}-\mathrm{NMR}\left(100 \mathrm{MHz}, \mathrm{CDCl}_{3}\right)$ :

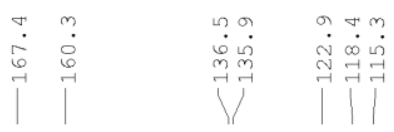

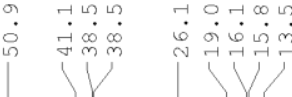

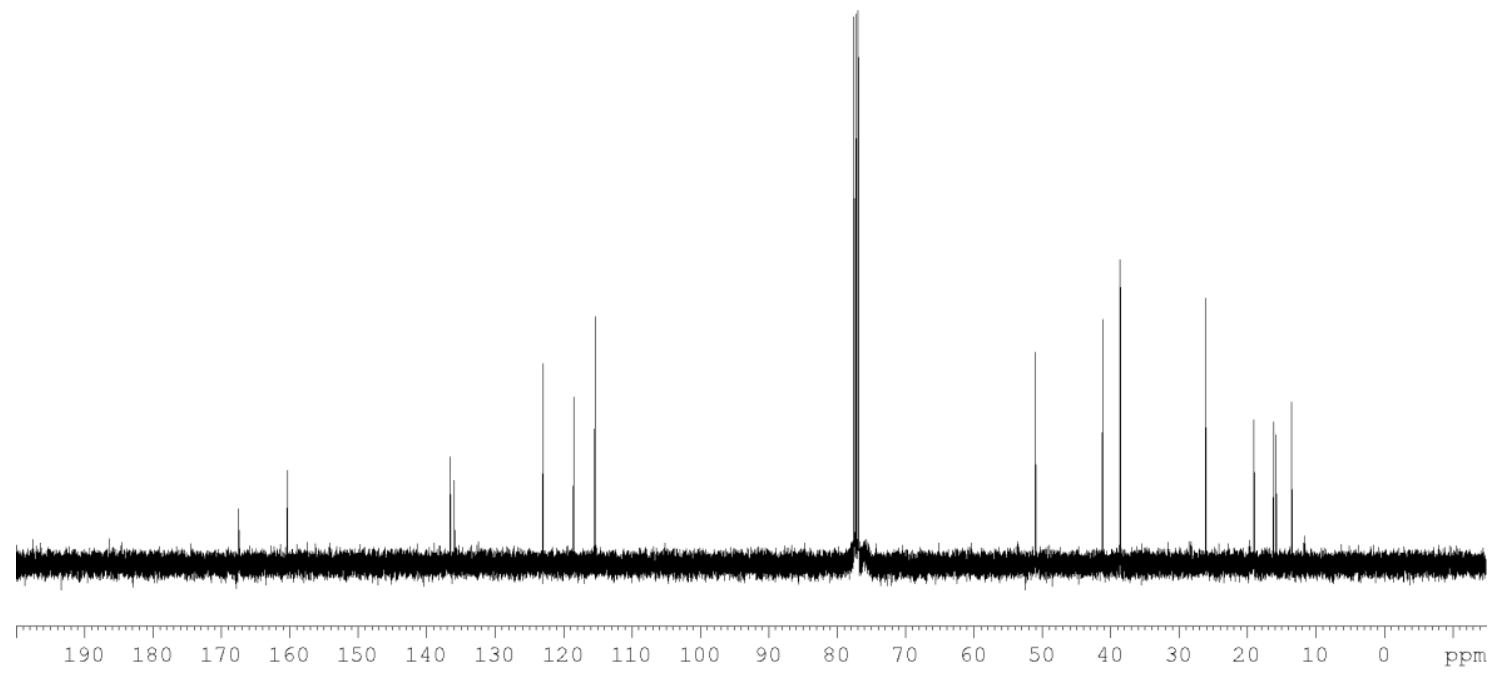


${ }^{1} \mathrm{H}-\mathrm{NMR}\left(400 \mathrm{MHz}, \mathrm{CDCl}_{3}\right.$ ):

7.

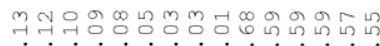

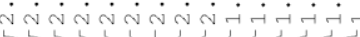

$\overbrace{}^{1} \overbrace{\mathrm{OH}}$

S6
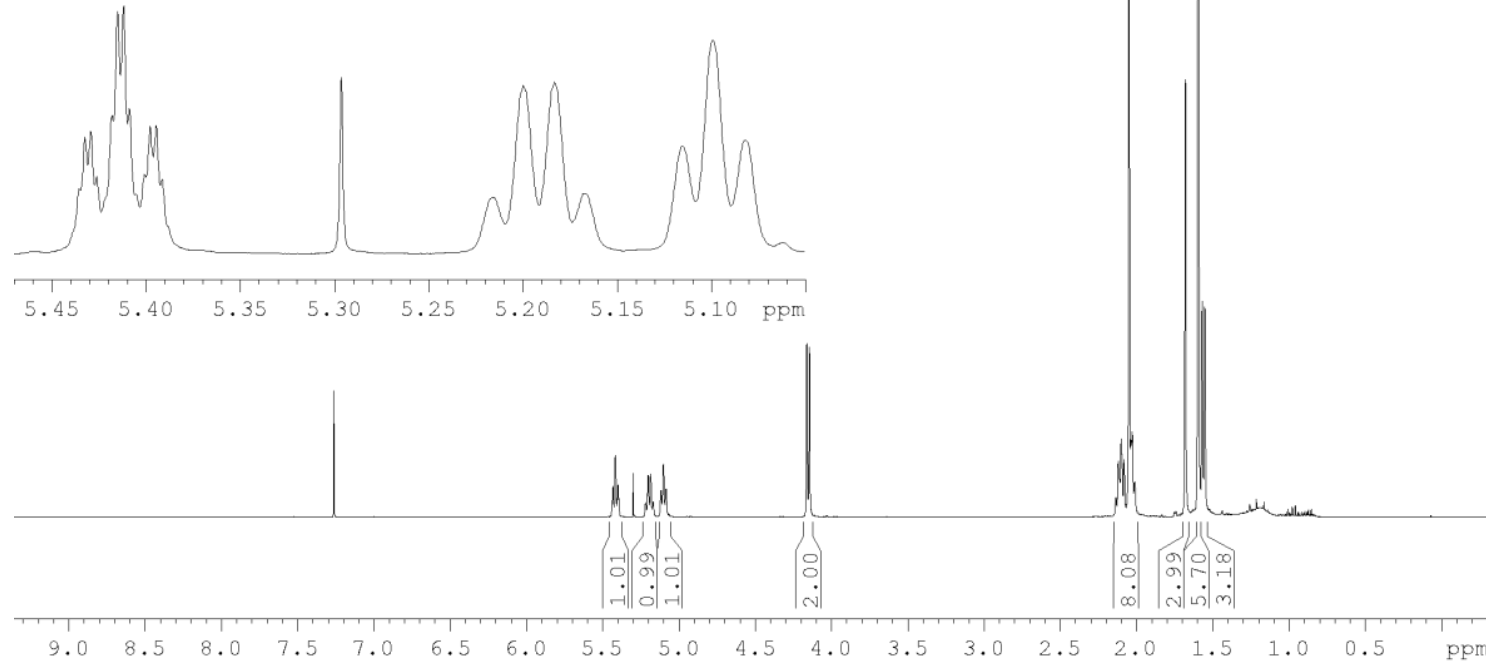

${ }^{13} \mathrm{C}-\mathrm{NMR}\left(100 \mathrm{MHz}, \mathrm{CDCl}_{3}\right)$ :
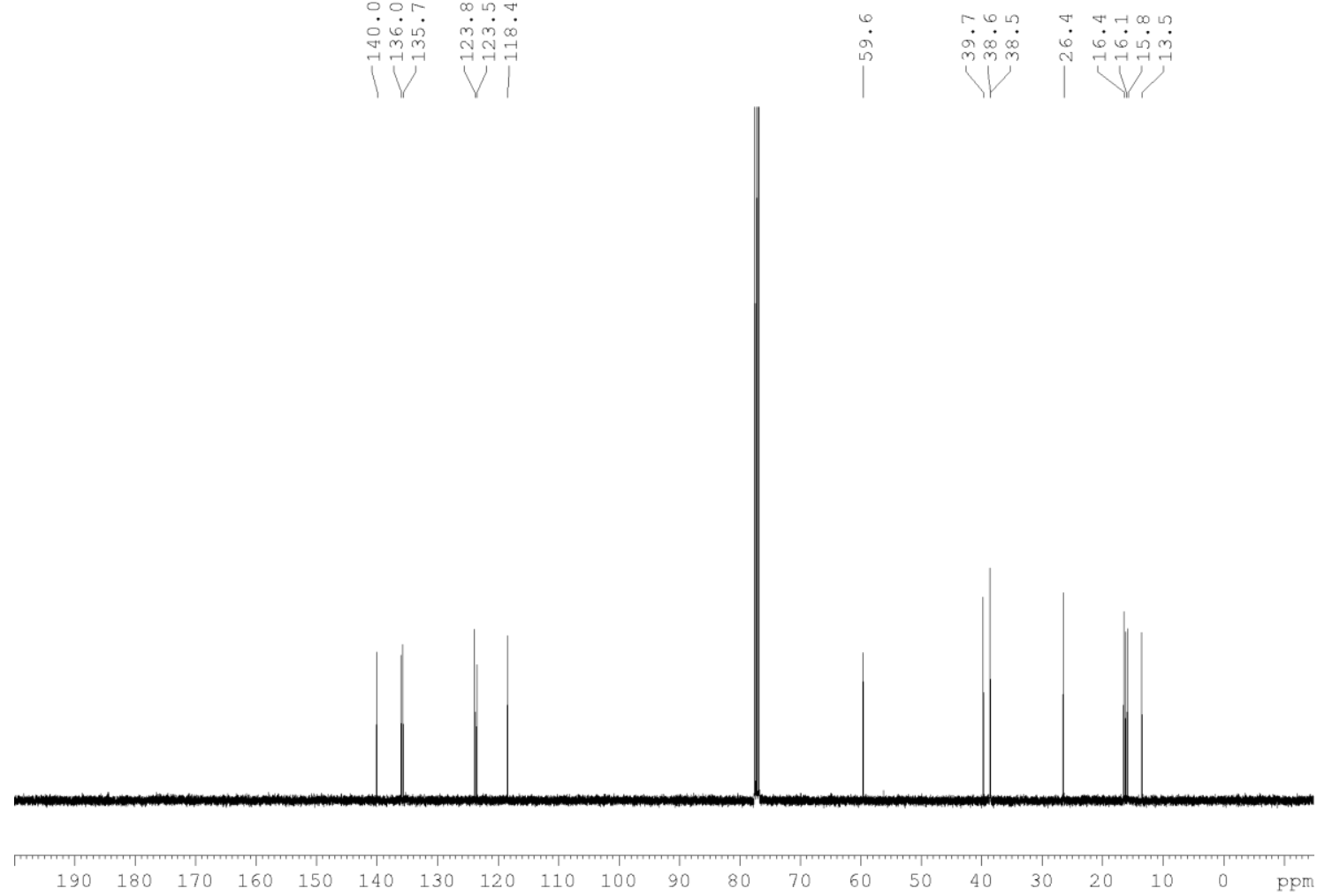
${ }^{1} \mathrm{H}-\mathrm{NMR}\left(400 \mathrm{MHz}, \mathrm{D}_{2} \mathrm{O}\right)$ :

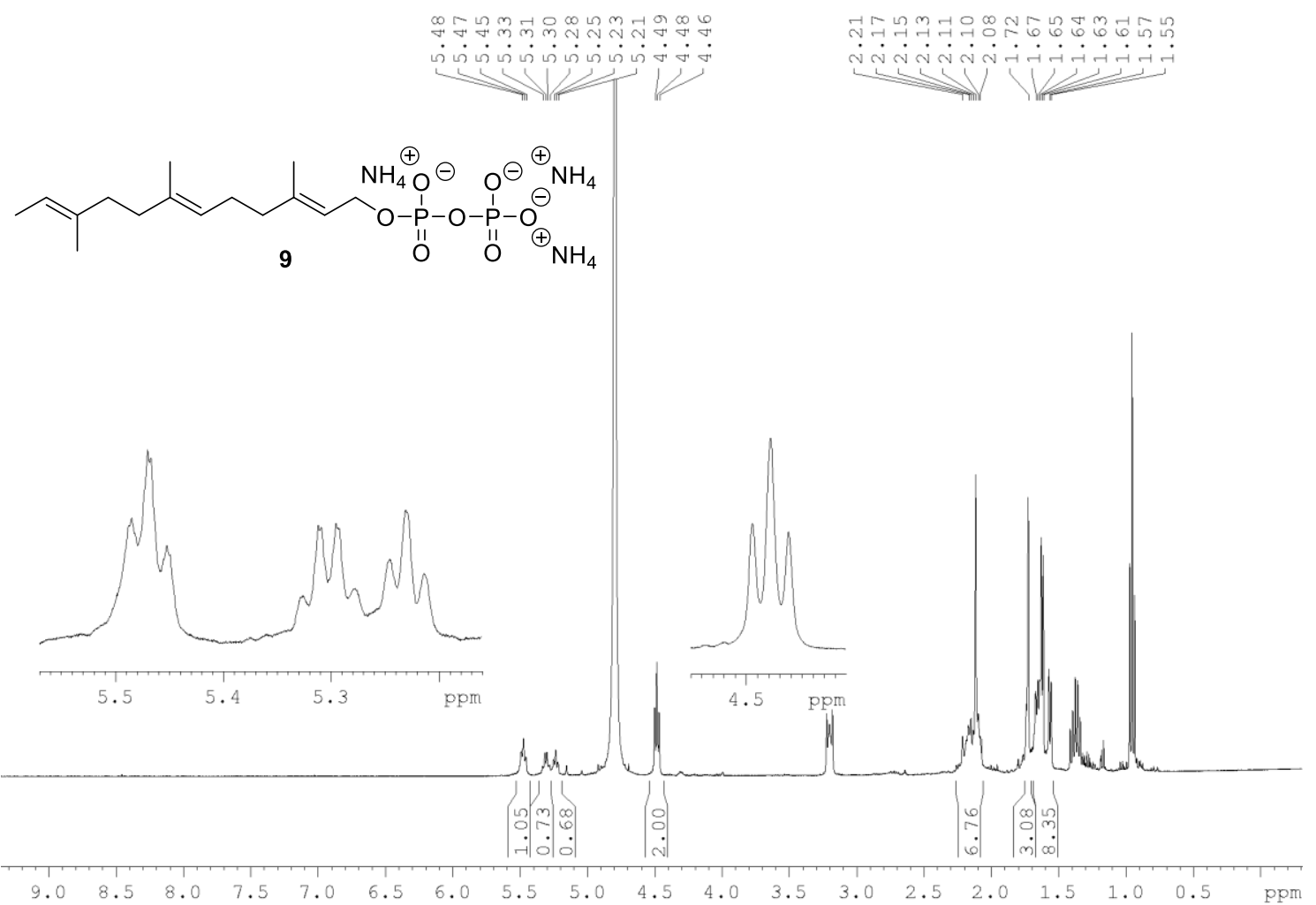

${ }^{13} \mathrm{C}-\mathrm{NMR}\left(100 \mathrm{MHz}, \mathrm{D}_{2} \mathrm{O}\right)$ :

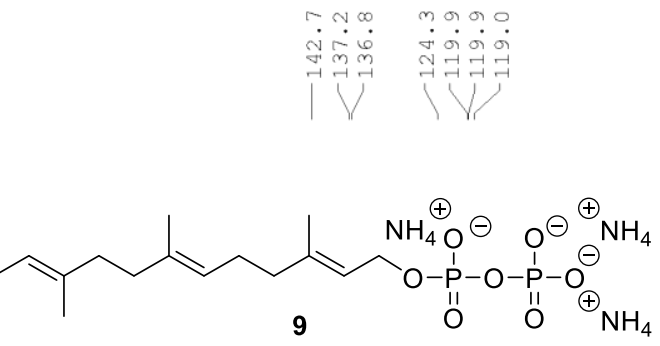


${ }^{1} \mathrm{H}-\mathrm{NMR}\left(400 \mathrm{MHz}, \mathrm{CDCl}_{3}\right.$ ):

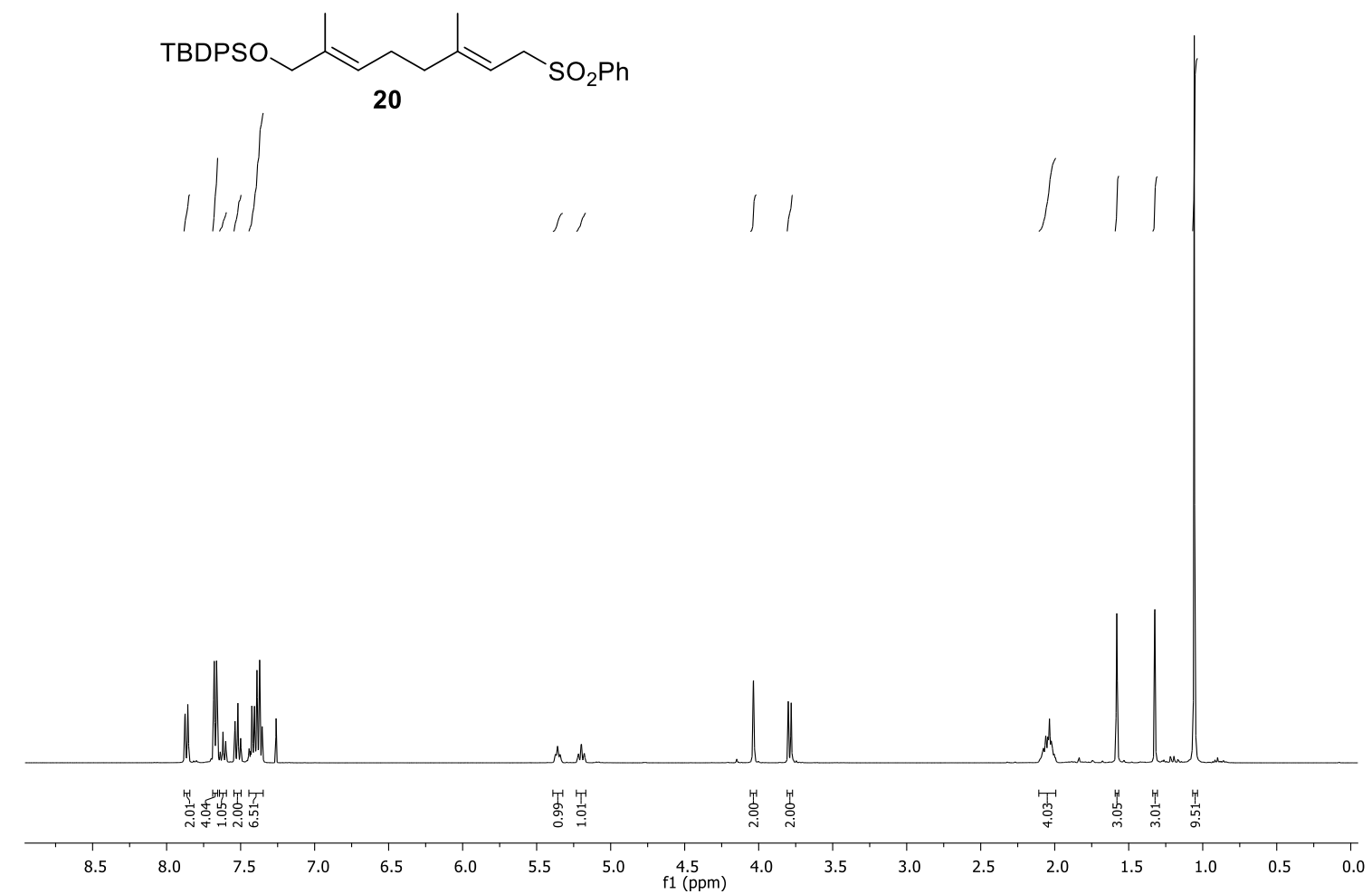

${ }^{13} \mathrm{C}-\mathrm{NMR}\left(100 \mathrm{MHz}, \mathrm{CDCl}_{3}\right)$ :

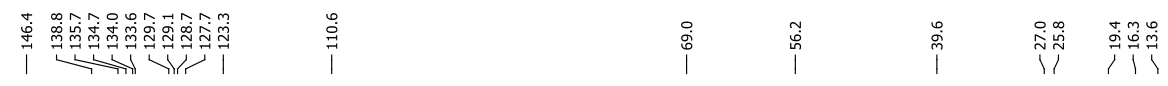
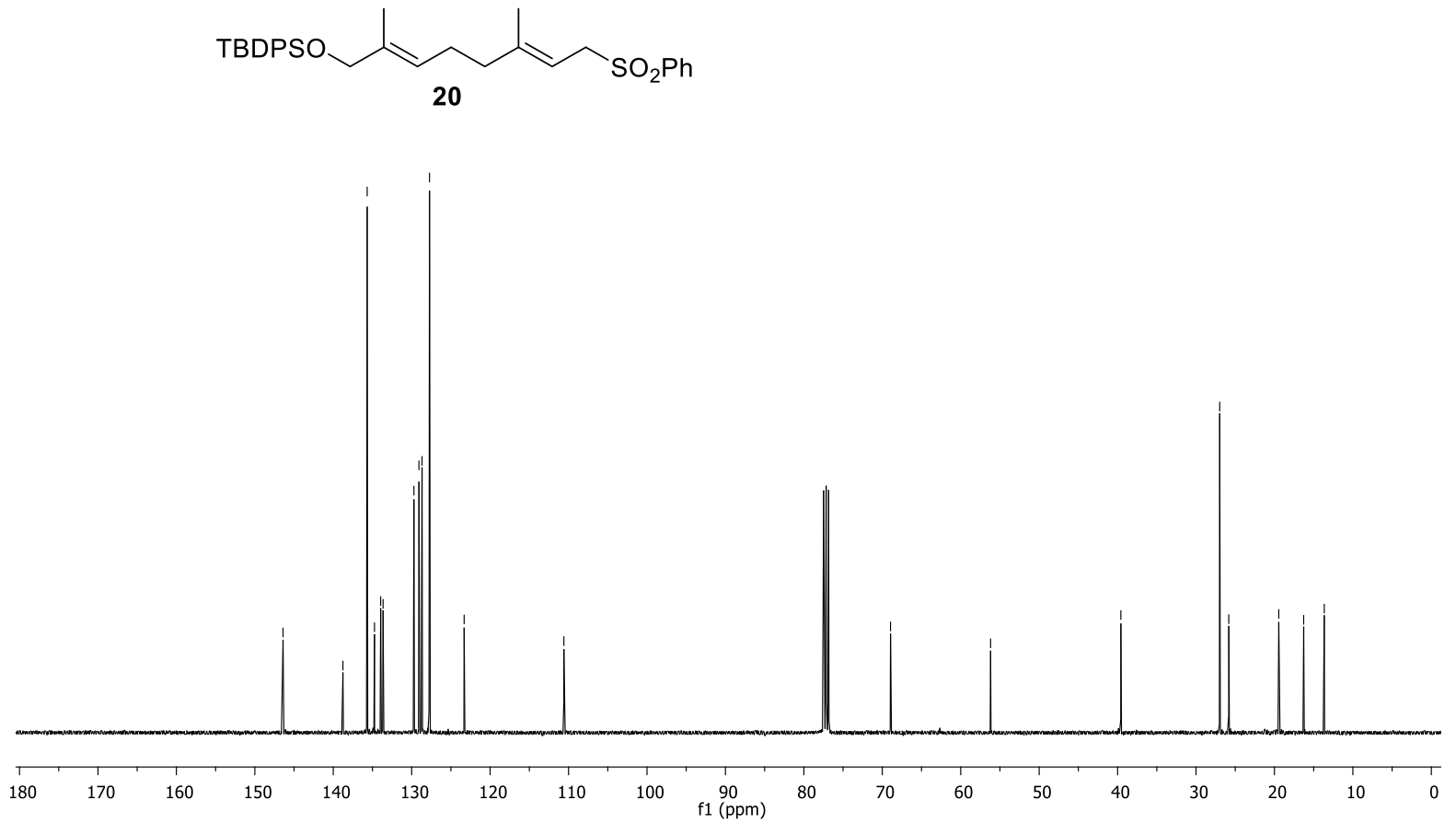
${ }^{1} \mathrm{H}-\mathrm{NMR}\left(400 \mathrm{MHz}, \mathrm{CDCl}_{3}\right)$ :

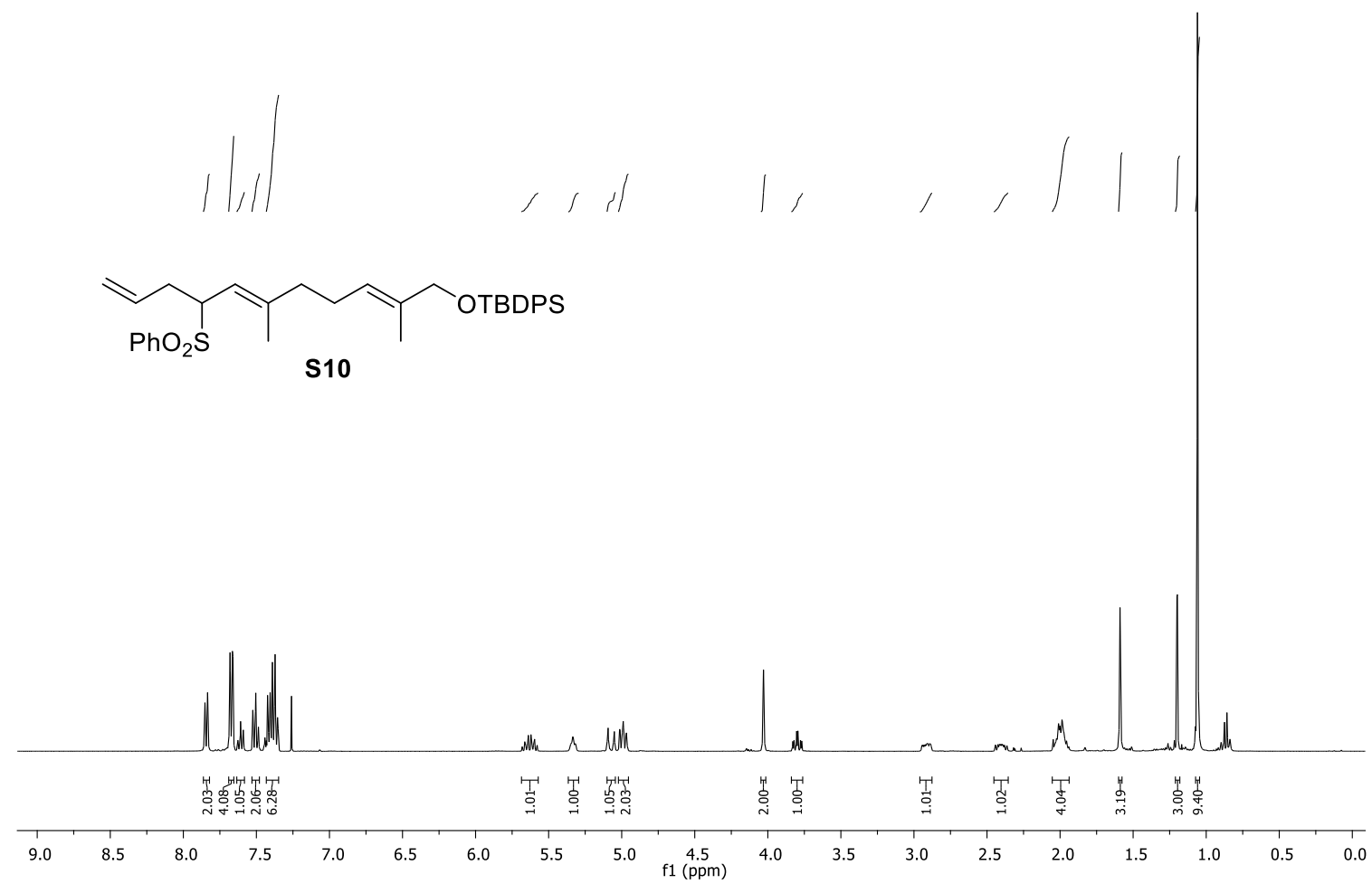

${ }^{13} \mathrm{C}-\mathrm{NMR}\left(100 \mathrm{MHz}, \mathrm{CDCl}_{3}\right)$ :

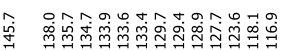

눈

金苦
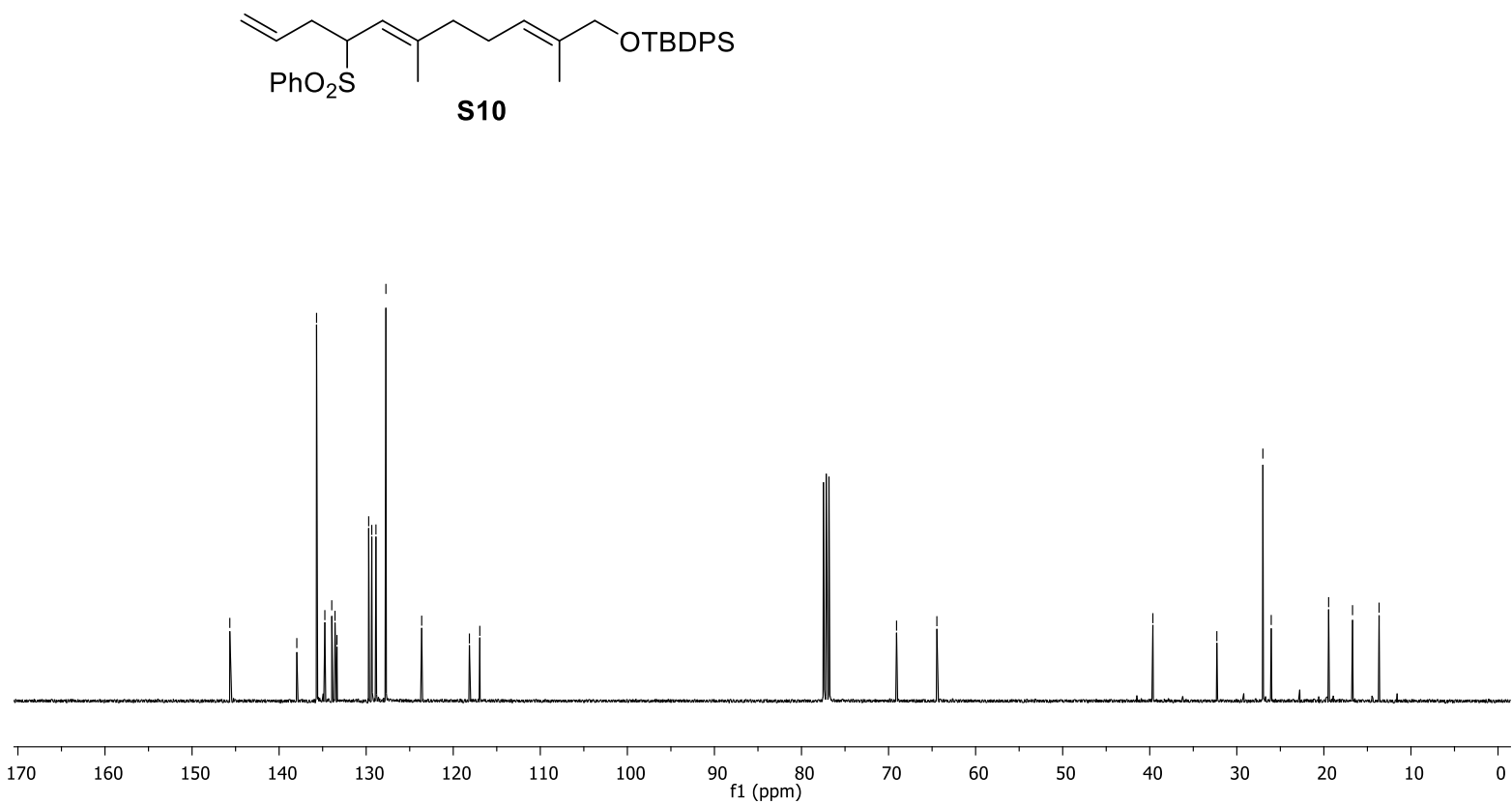
${ }^{1} \mathrm{H}-\mathrm{NMR}\left(400 \mathrm{MHz}, \mathrm{CDCl}_{3}\right)$ :
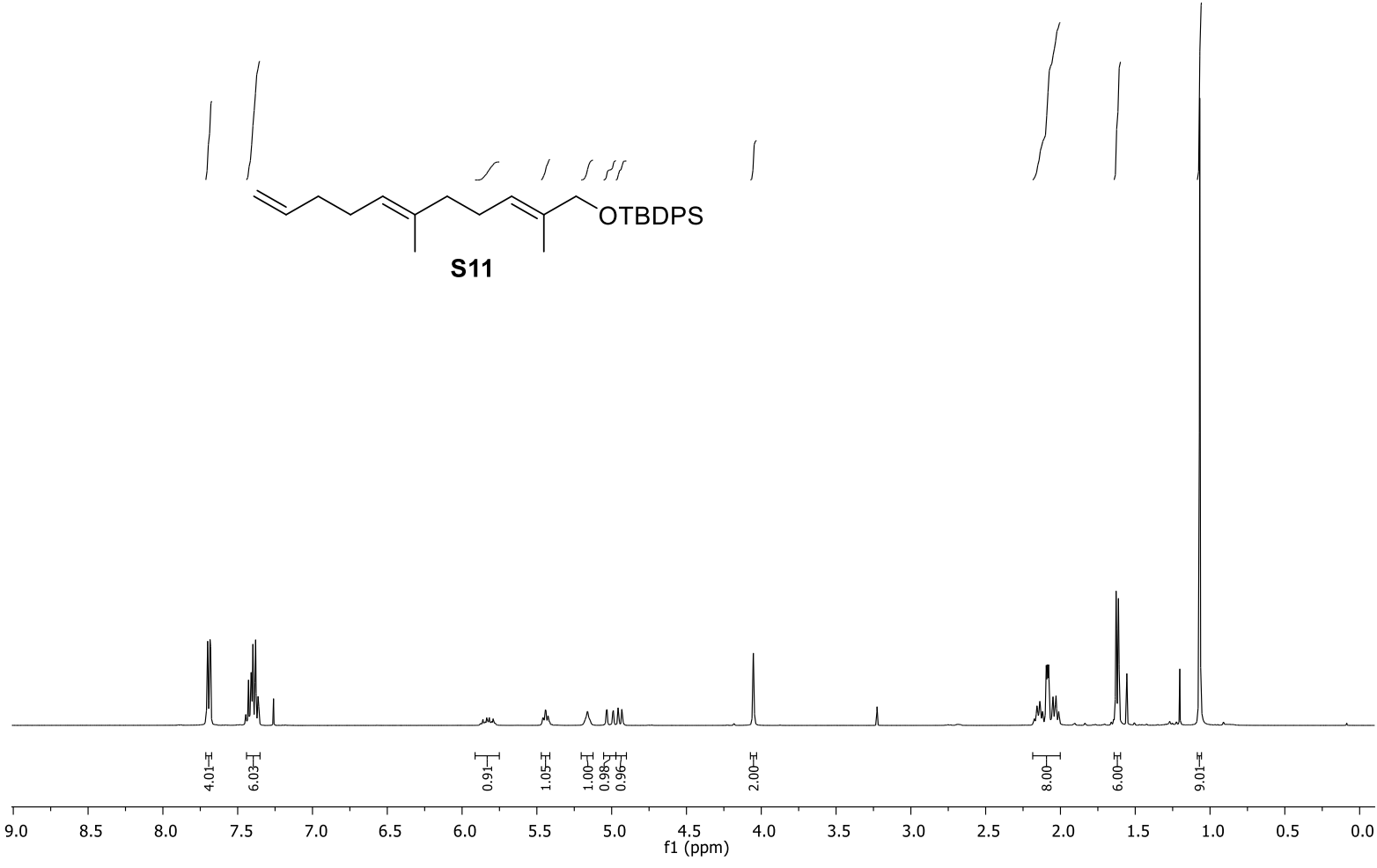

${ }^{13} \mathrm{C}-\mathrm{NMR}\left(100 \mathrm{MHz}, \mathrm{CDCl}_{3}\right)$ :
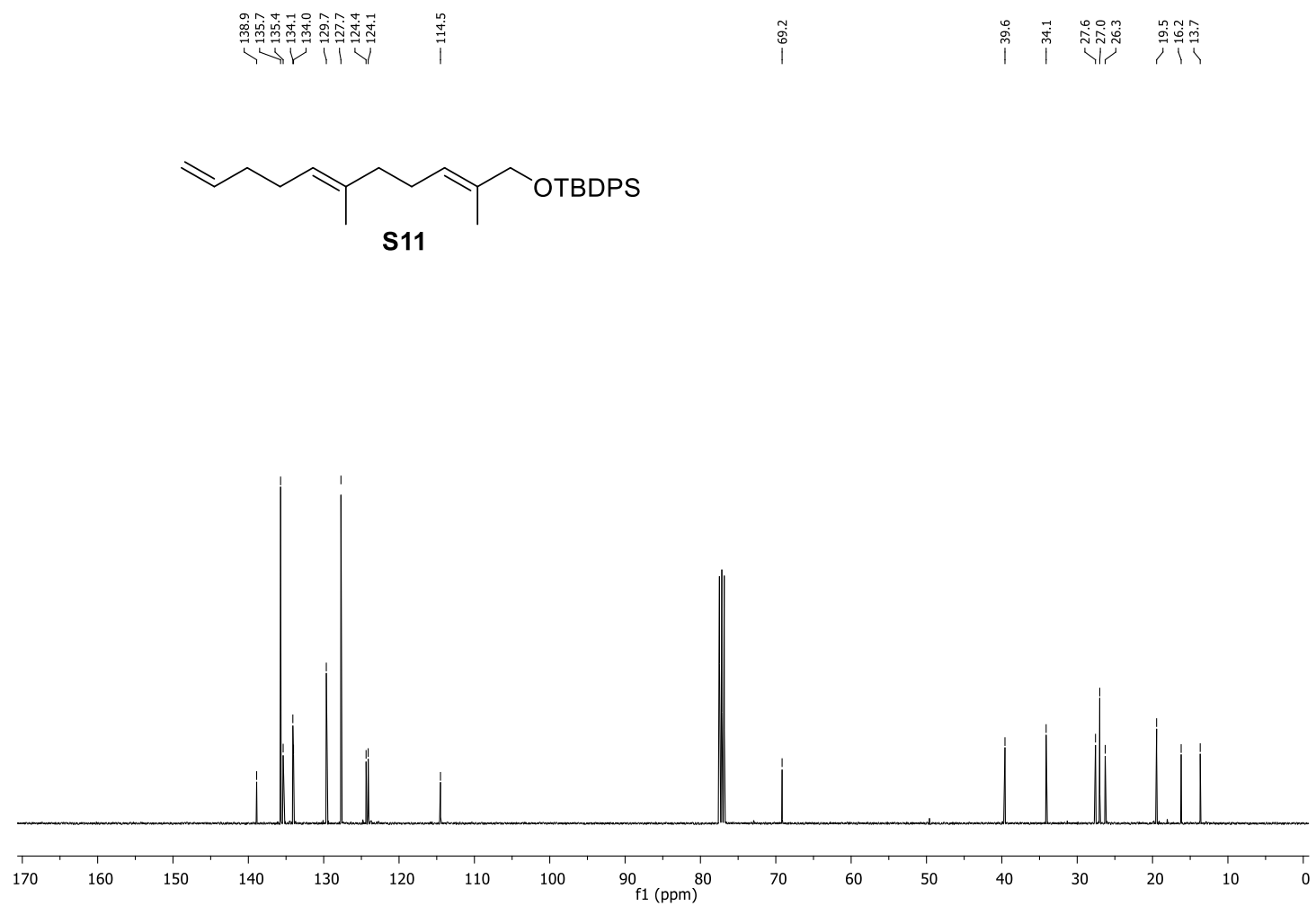
${ }^{1} \mathrm{H}-\mathrm{NMR}\left(400 \mathrm{MHz}, \mathrm{CDCl}_{3}\right.$ ):<smiles>C=CCC/C=C(\C)CC/C=C(\C)CO</smiles>

21
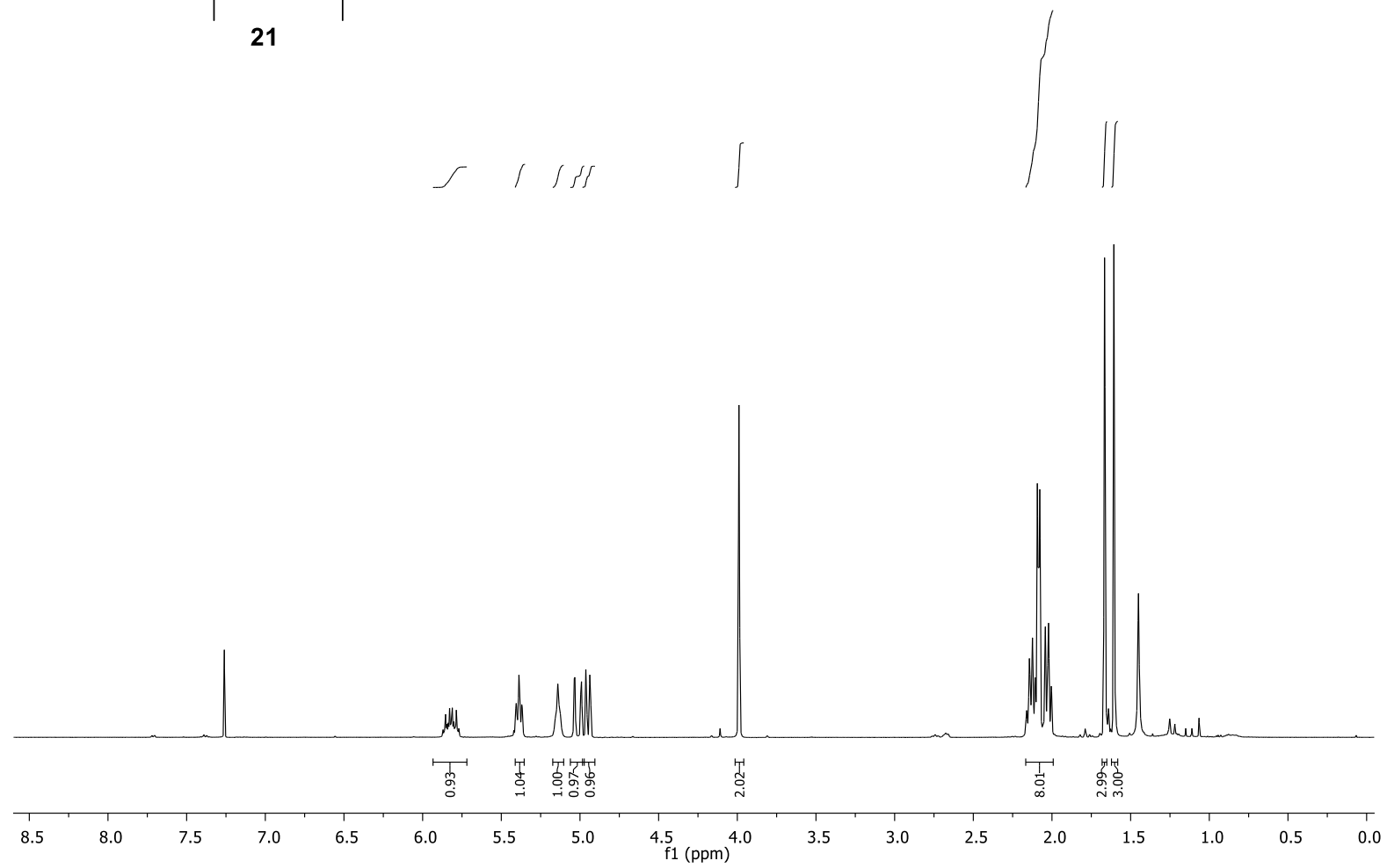

${ }^{13} \mathrm{C}-\mathrm{NMR}\left(100 \mathrm{MHz}, \mathrm{CDCl}_{3}\right)$ :
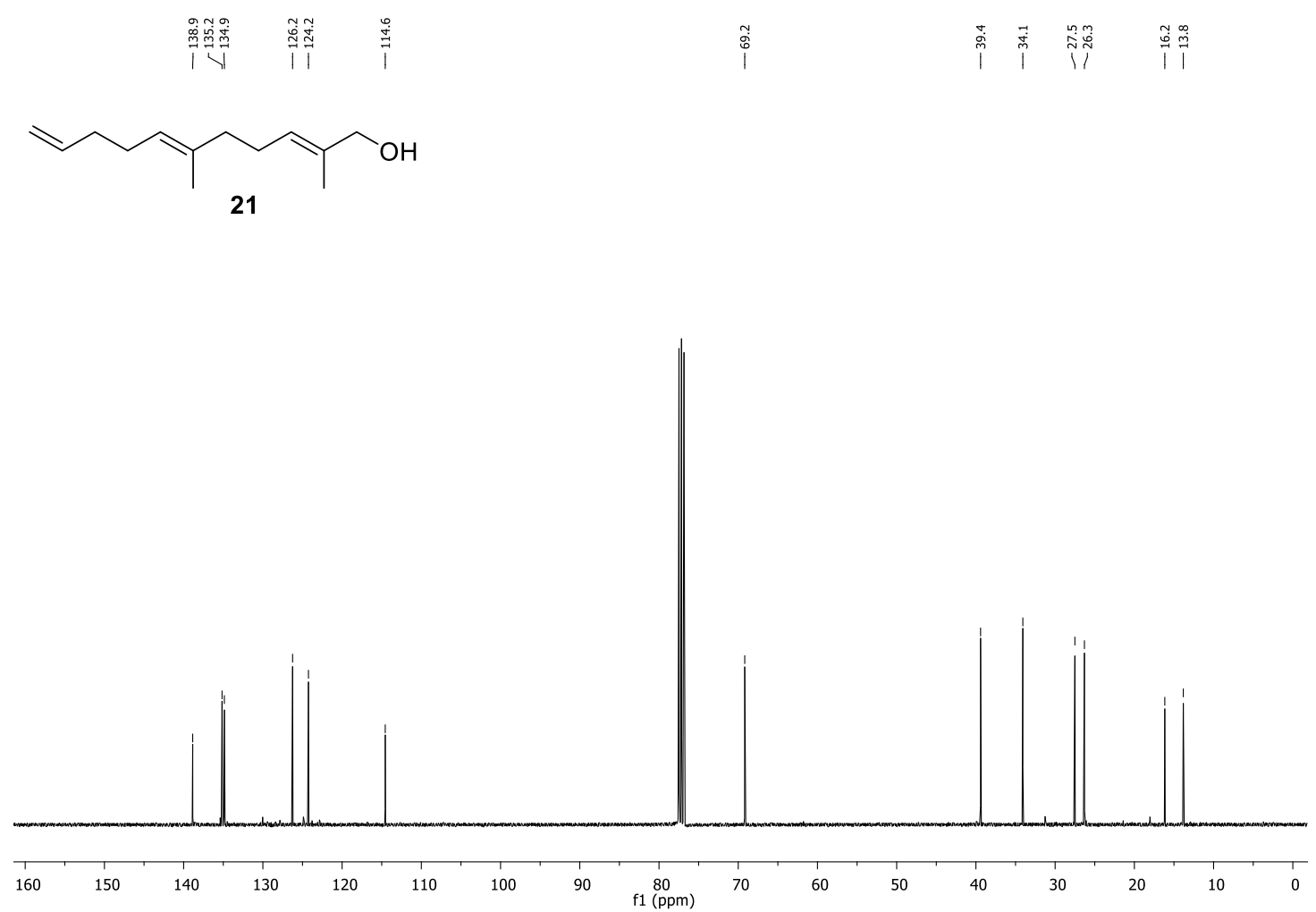
${ }^{1} \mathrm{H}-\mathrm{NMR}\left(400 \mathrm{MHz}, \mathrm{D}_{2} \mathrm{O}\right)$ :

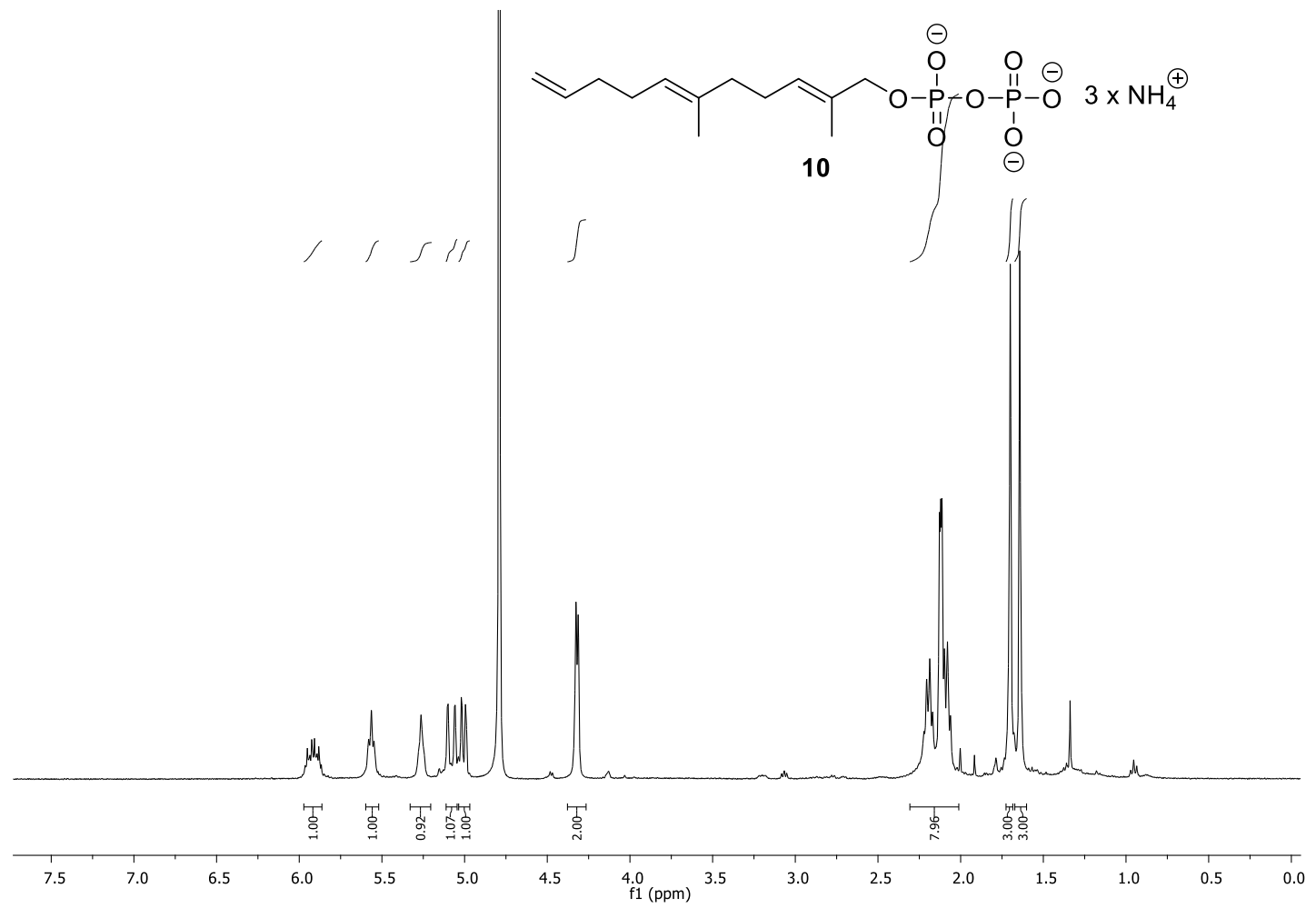

${ }^{13} \mathrm{C}-\mathrm{NMR}\left(100 \mathrm{MHz}, \mathrm{D}_{2} \mathrm{O}\right)$ :

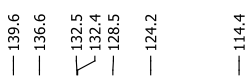
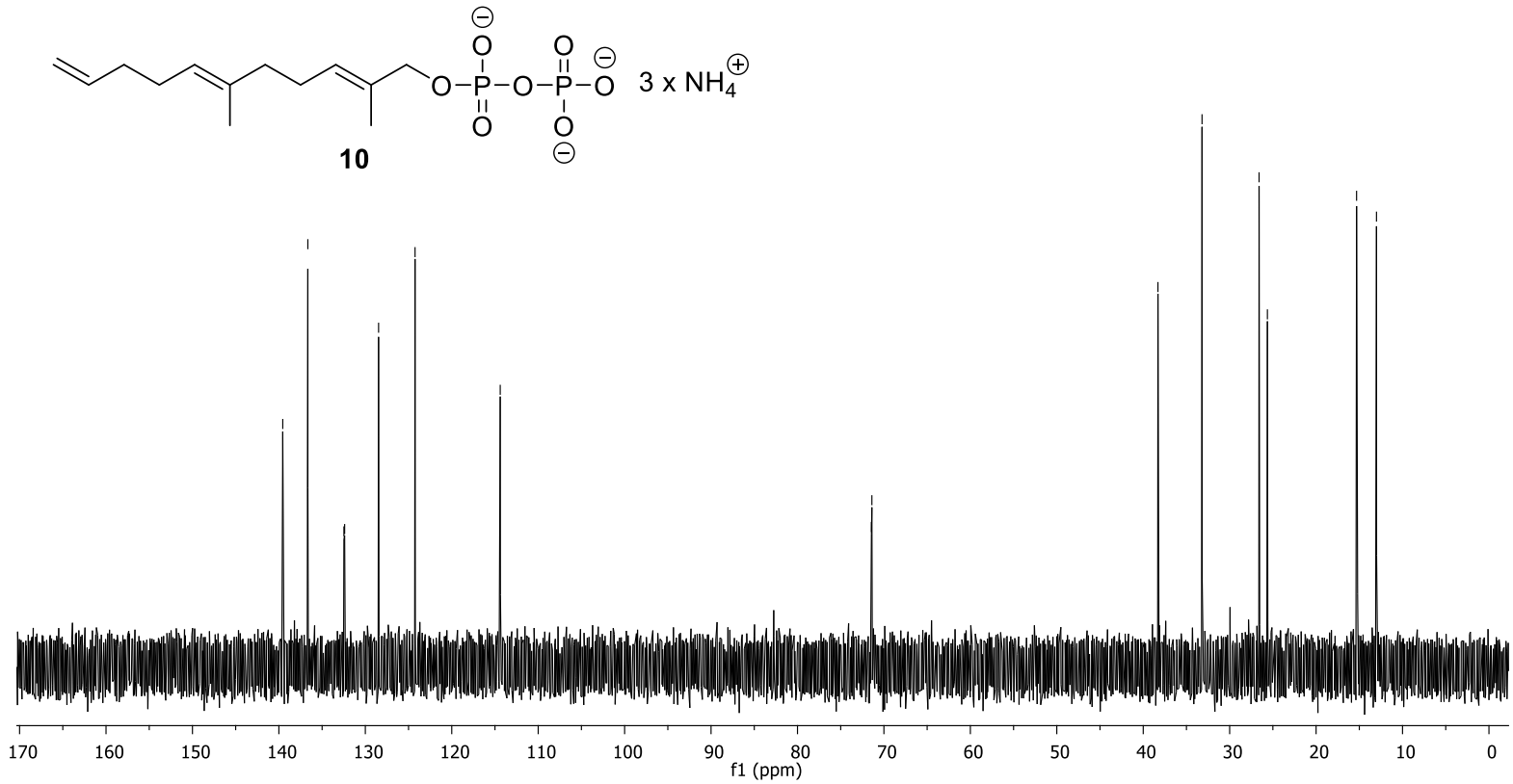
${ }^{1} \mathrm{H}-\mathrm{NMR}\left(400 \mathrm{MHz}, \mathrm{CDCl}_{3}\right.$ ):

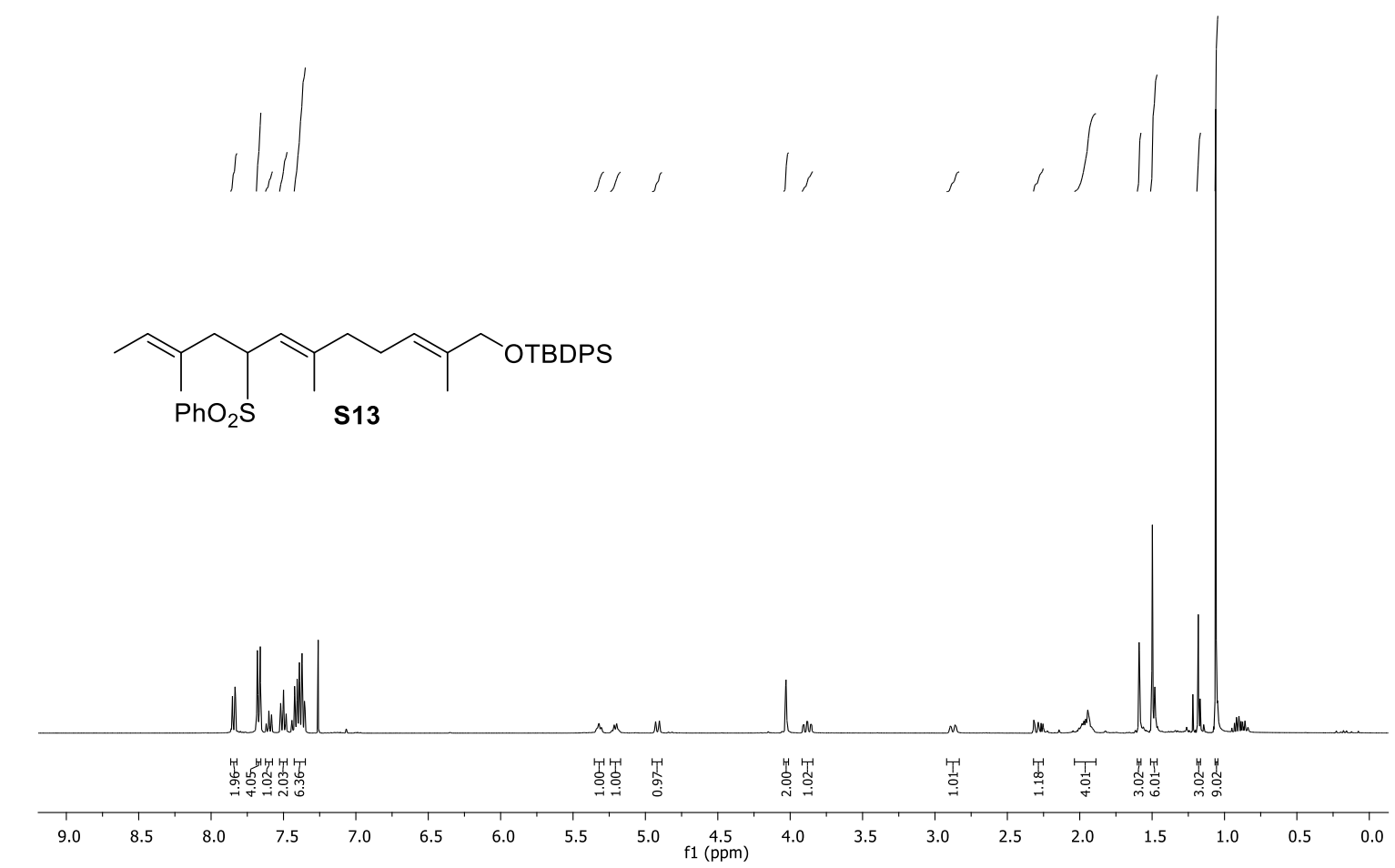

${ }^{13} \mathrm{C}-\mathrm{NMR}\left(100 \mathrm{MHz}, \mathrm{CDCl}_{3}\right)$ :

|

궁

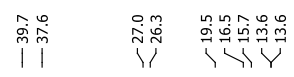
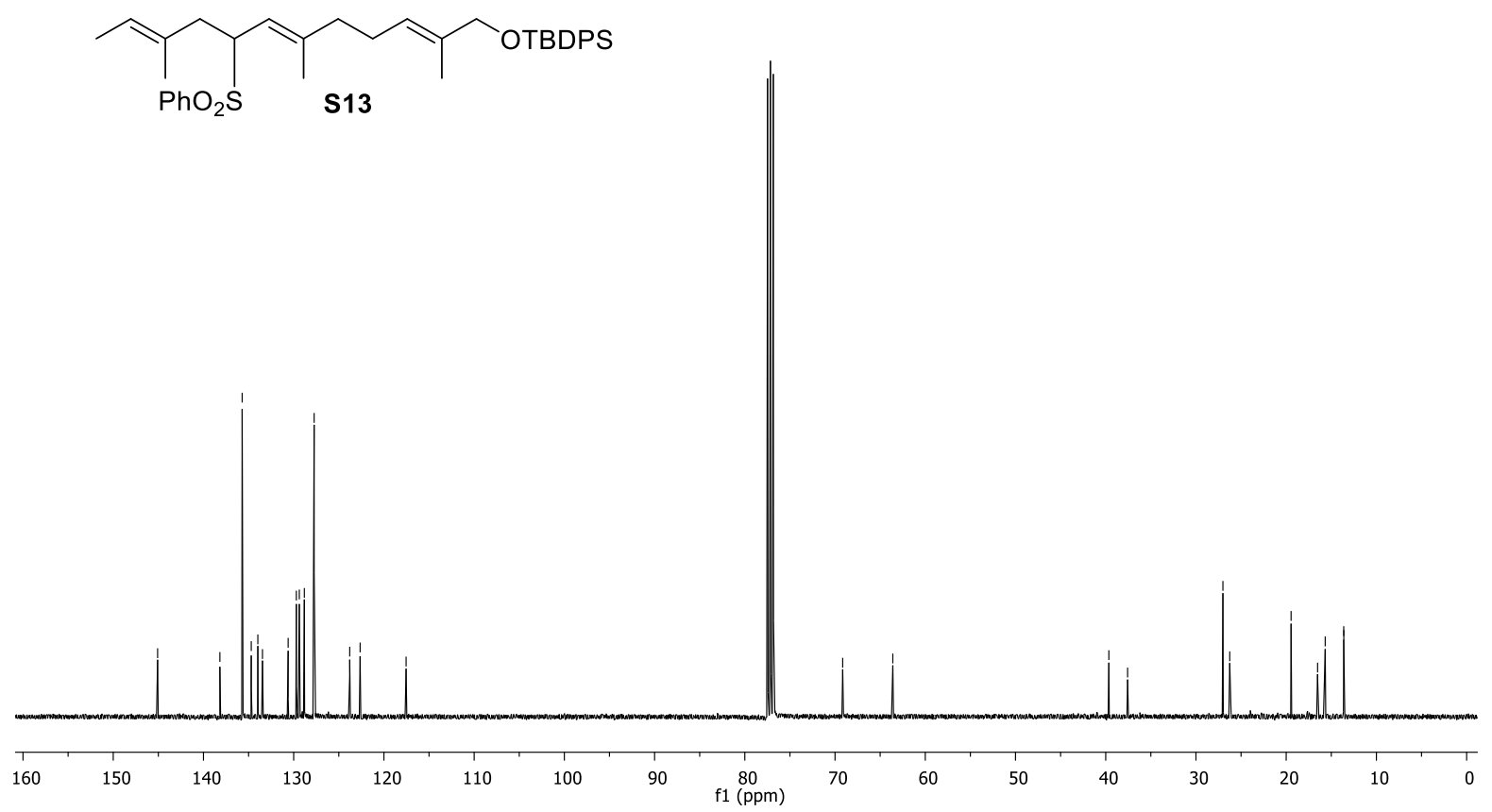
${ }^{1} \mathrm{H}-\mathrm{NMR}\left(400 \mathrm{MHz}, \mathrm{CDCl}_{3}\right.$ ):

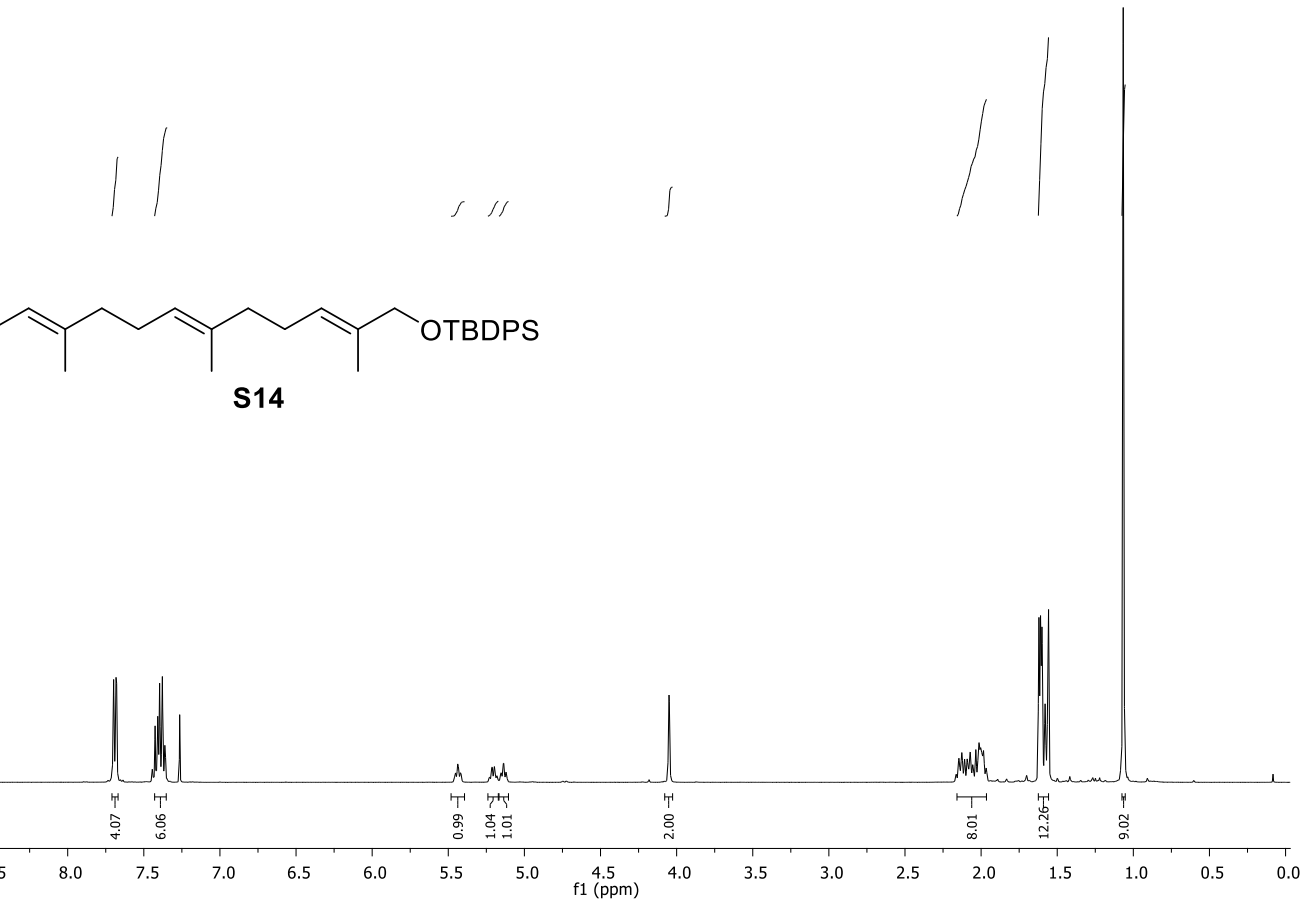

${ }^{13} \mathrm{C}-\mathrm{NMR}\left(100 \mathrm{MHz}, \mathrm{CDCl}_{3}\right)$ :
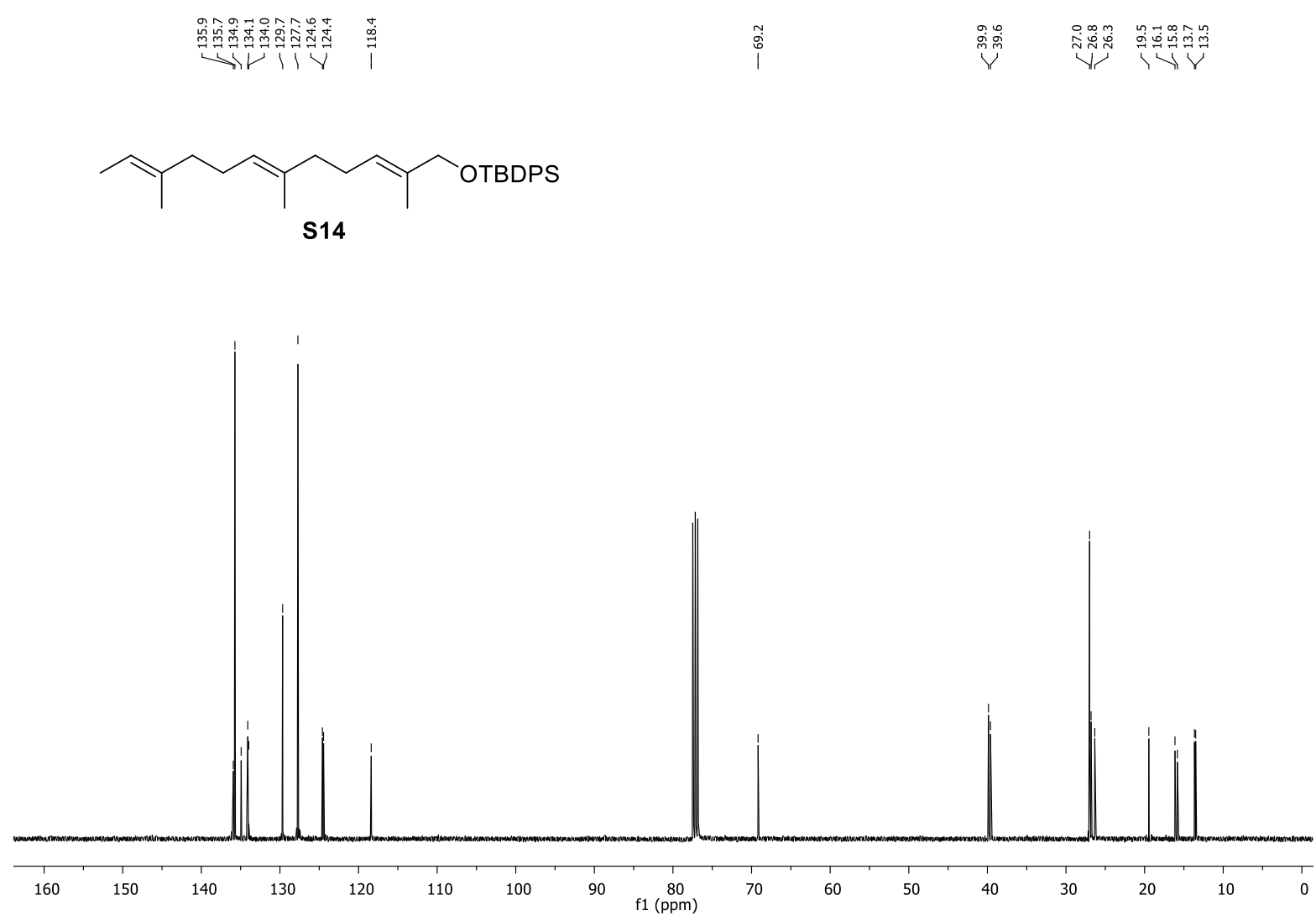
${ }^{1} \mathrm{H}-\mathrm{NMR}\left(400 \mathrm{MHz}, \mathrm{CDCl}_{3}\right.$ ):

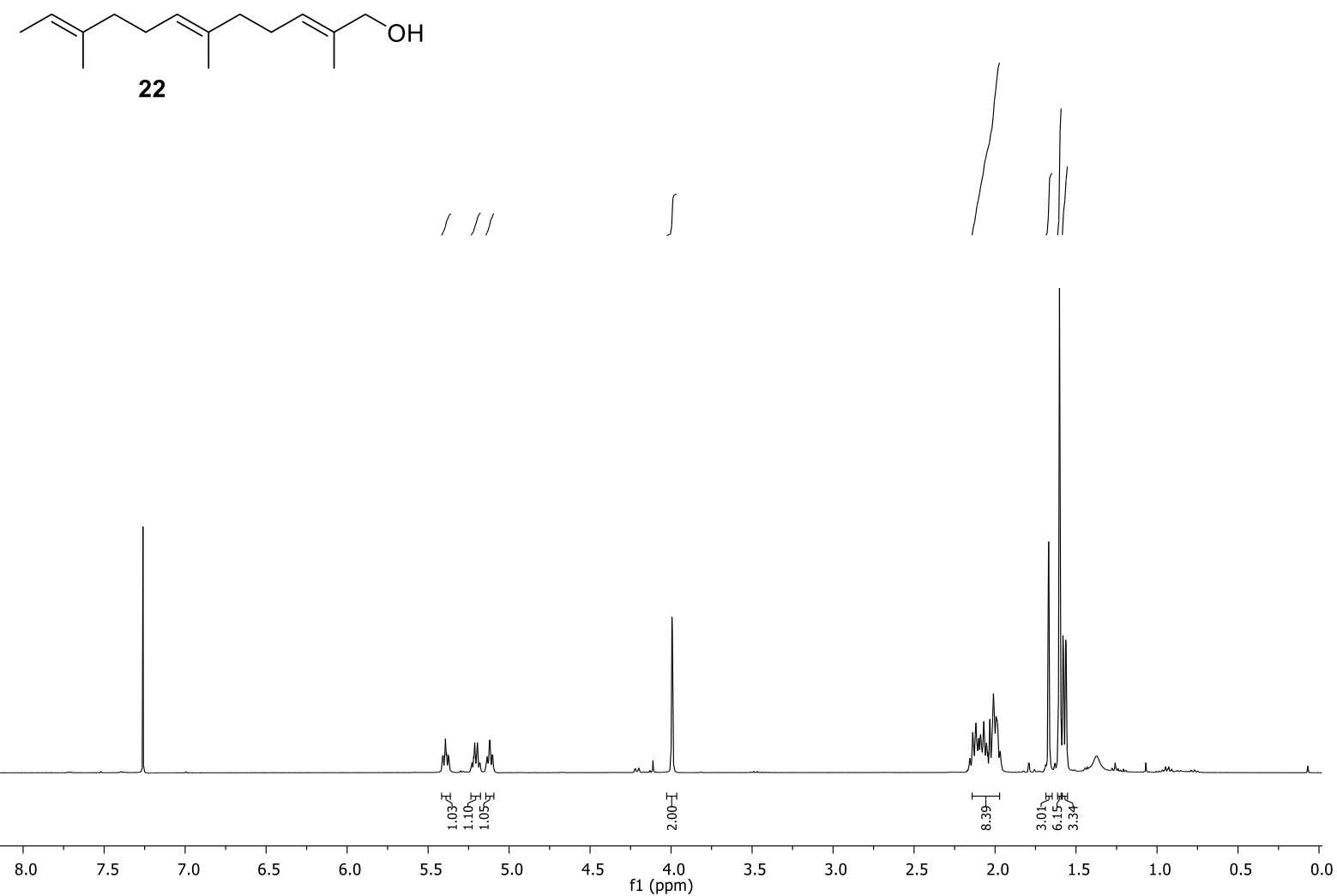

${ }^{13} \mathrm{C}-\mathrm{NMR}\left(100 \mathrm{MHz}, \mathrm{CDCl}_{3}\right)$ :

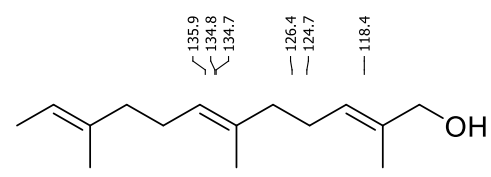

Vy

22

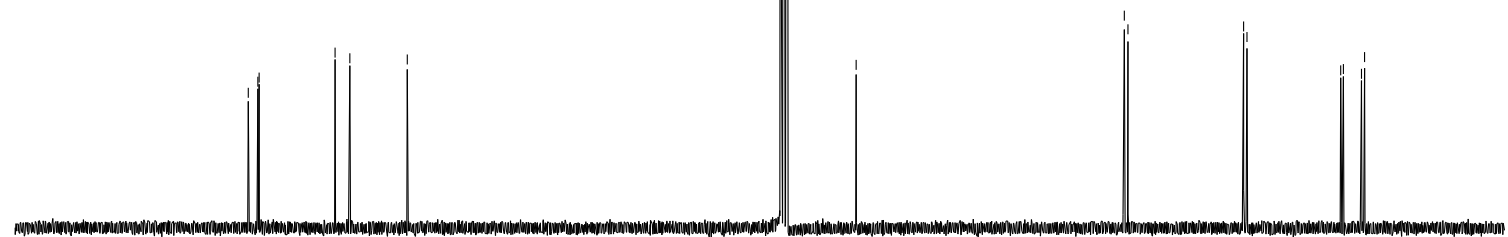

160

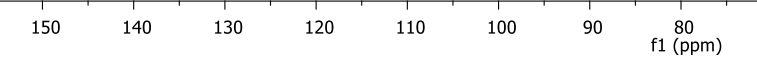

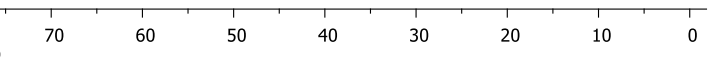


${ }^{1} \mathrm{H}-\mathrm{NMR}\left(400 \mathrm{MHz}, \mathrm{D}_{2} \mathrm{O}\right)$ :

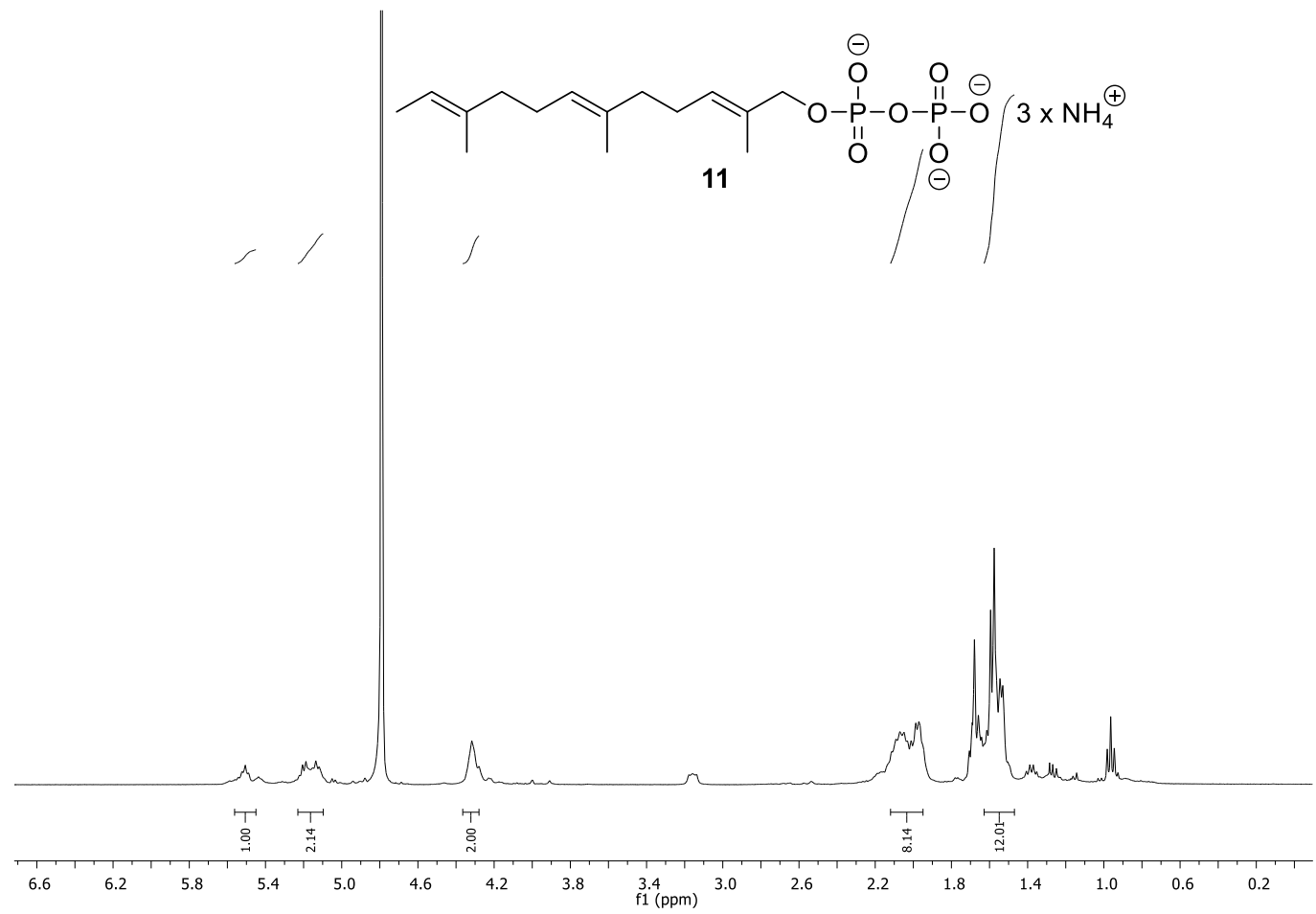

${ }^{13} \mathrm{C}-\mathrm{NMR}\left(100 \mathrm{MHz}, \mathrm{D}_{2} \mathrm{O}\right)$ :

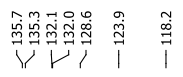
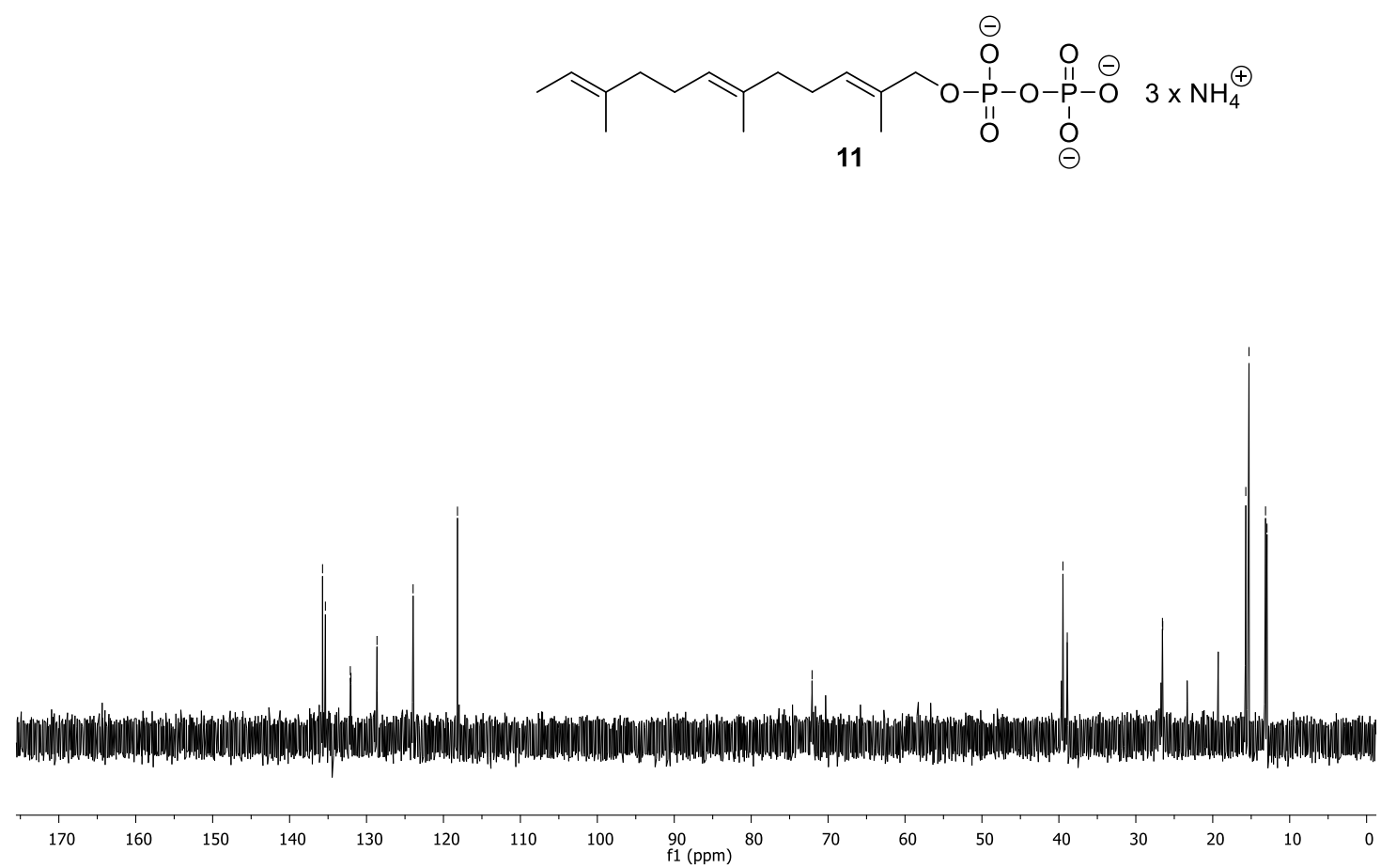
${ }^{1} \mathrm{H}-\mathrm{NMR}\left(400 \mathrm{MHz}, \mathrm{CDCl}_{3}\right.$ ):

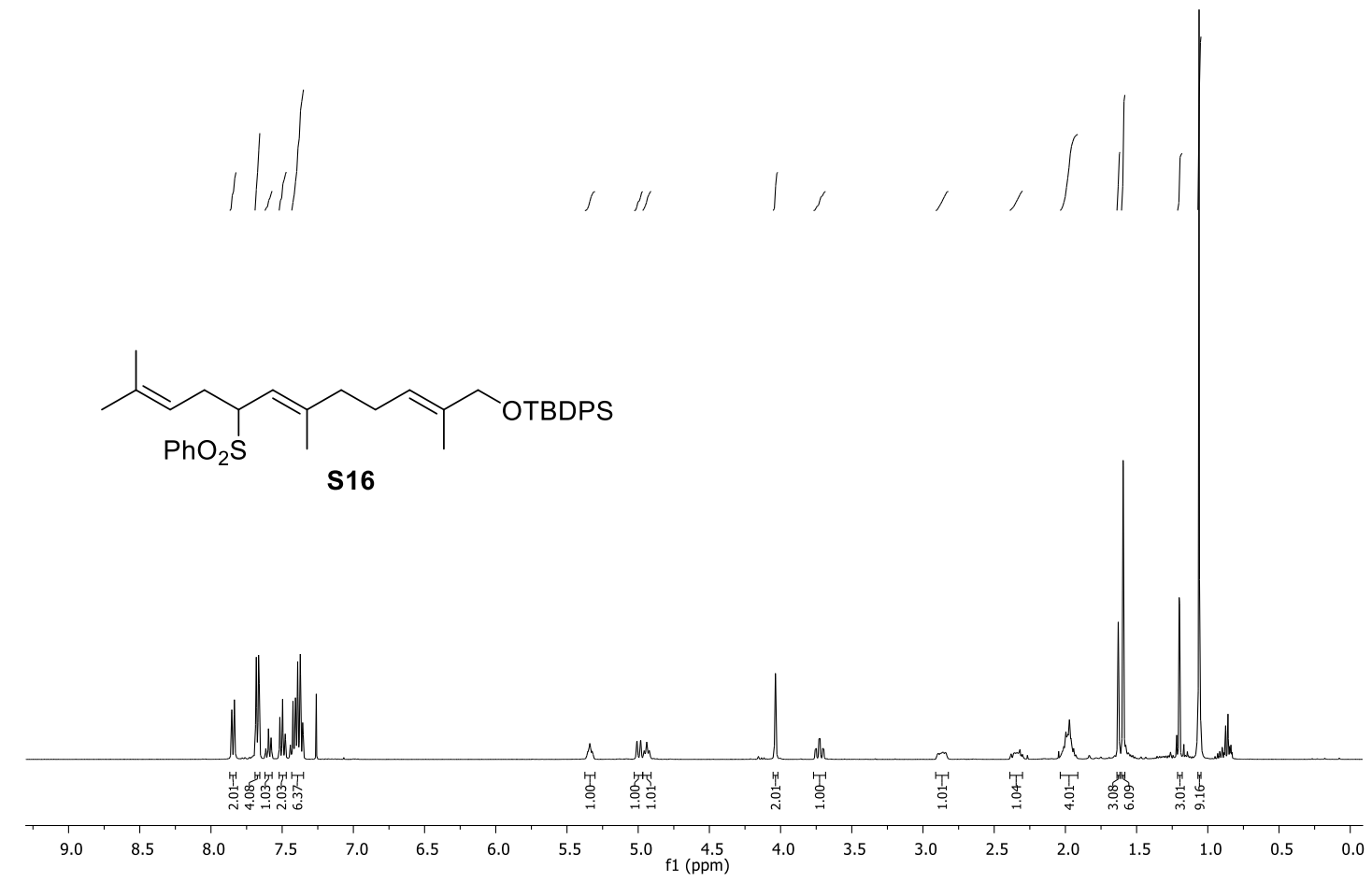

${ }^{13} \mathrm{C}-\mathrm{NMR}\left(100 \mathrm{MHz}, \mathrm{CDCl}_{3}\right)$ :

|

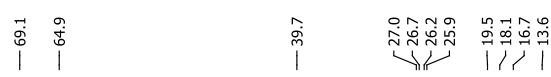
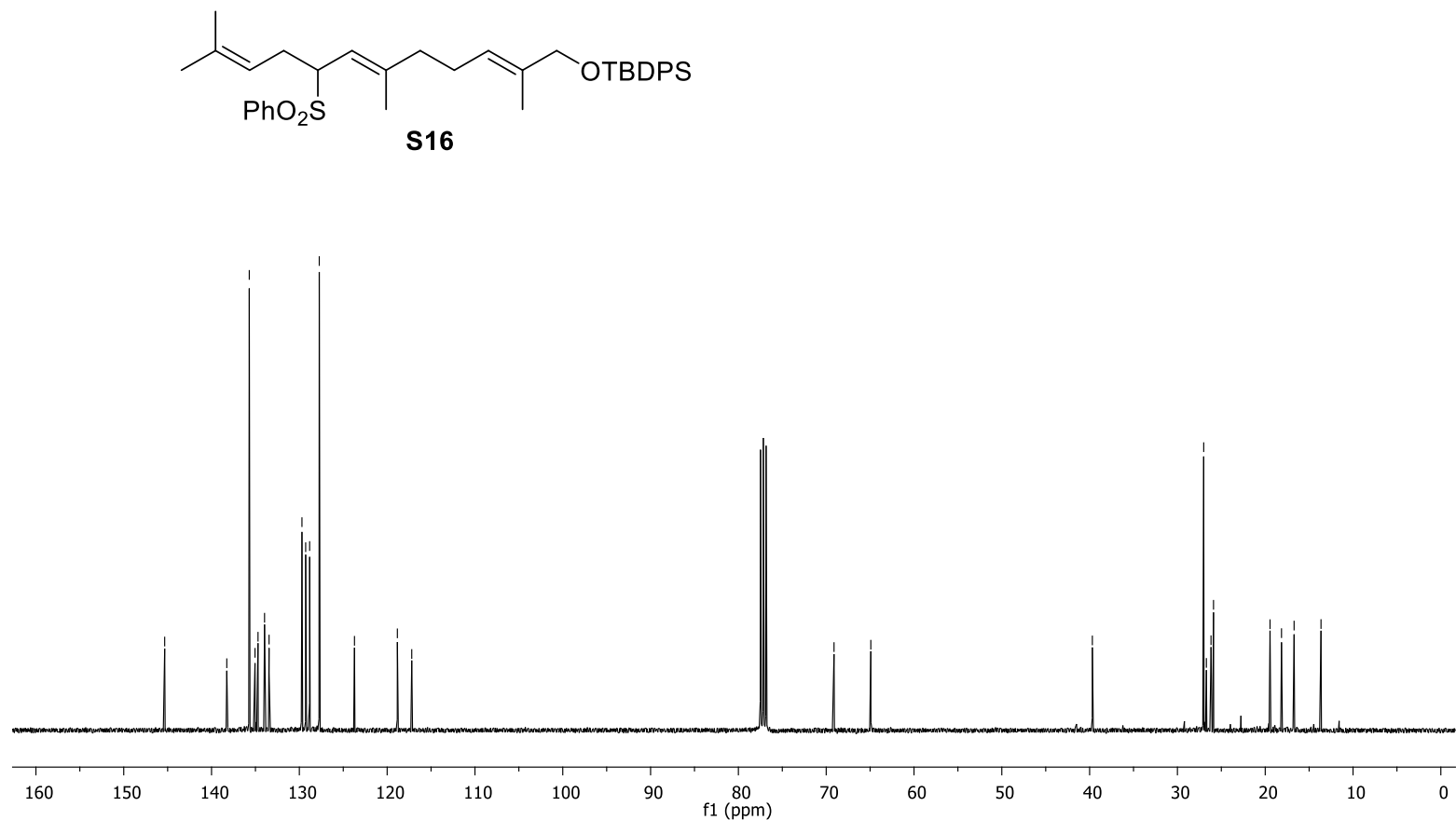
${ }^{1} \mathrm{H}-\mathrm{NMR}\left(400 \mathrm{MHz}, \mathrm{CDCl}_{3}\right.$ ):
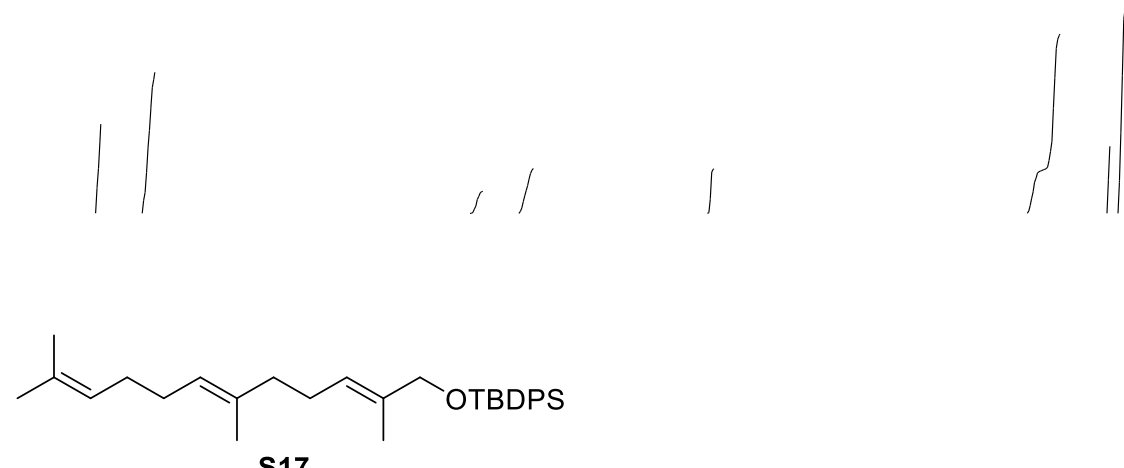

S17

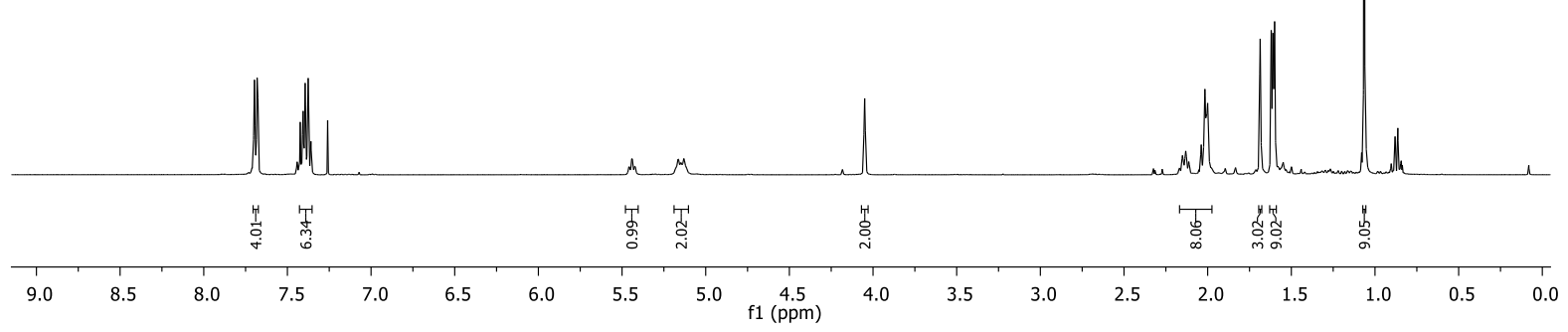


${ }^{1} \mathrm{H}-\mathrm{NMR}\left(400 \mathrm{MHz}, \mathrm{CDCl}_{3}\right.$ ):
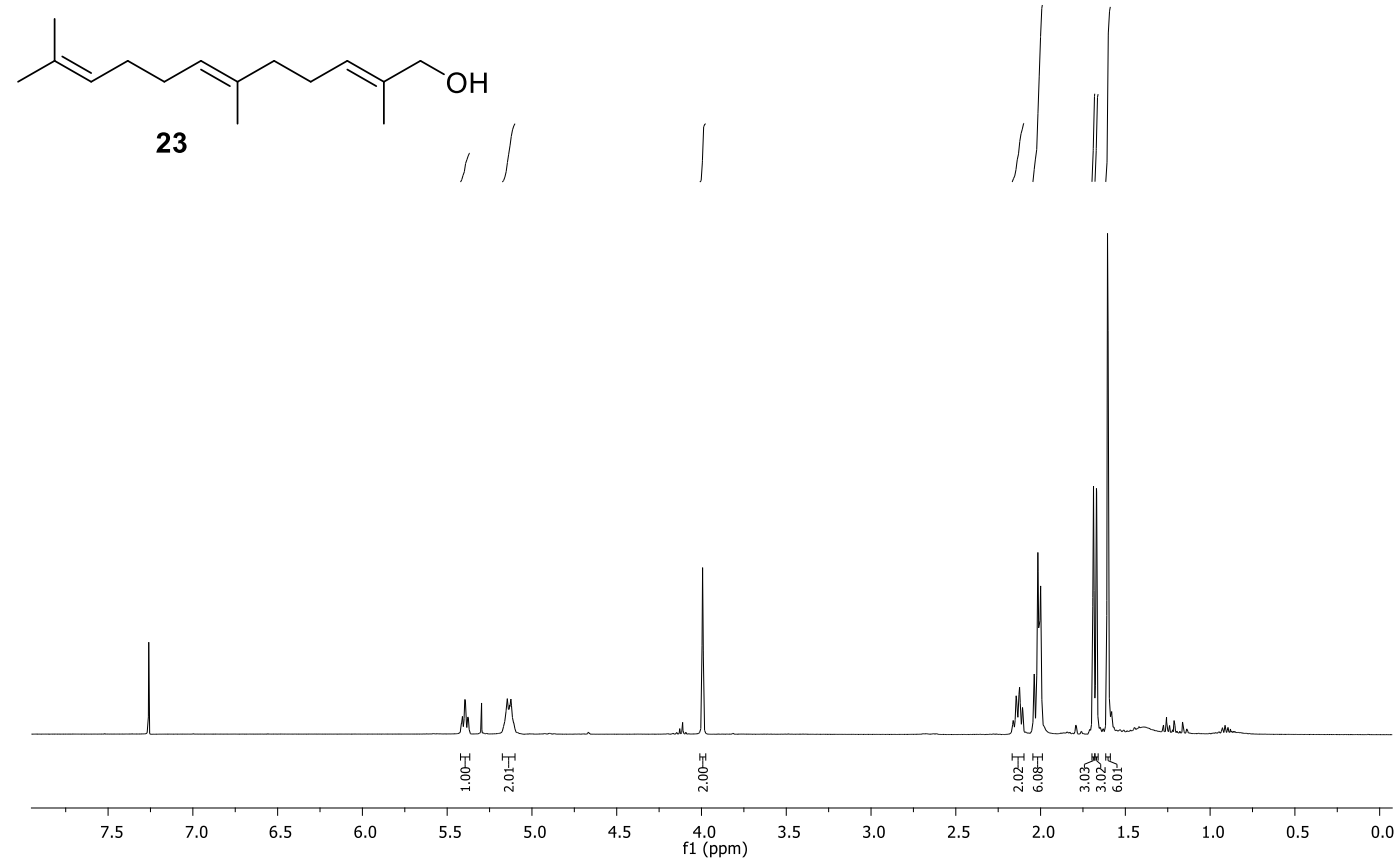

${ }^{13} \mathrm{C}-\mathrm{NMR}\left(100 \mathrm{MHz}, \mathrm{CDCl}_{3}\right)$ :
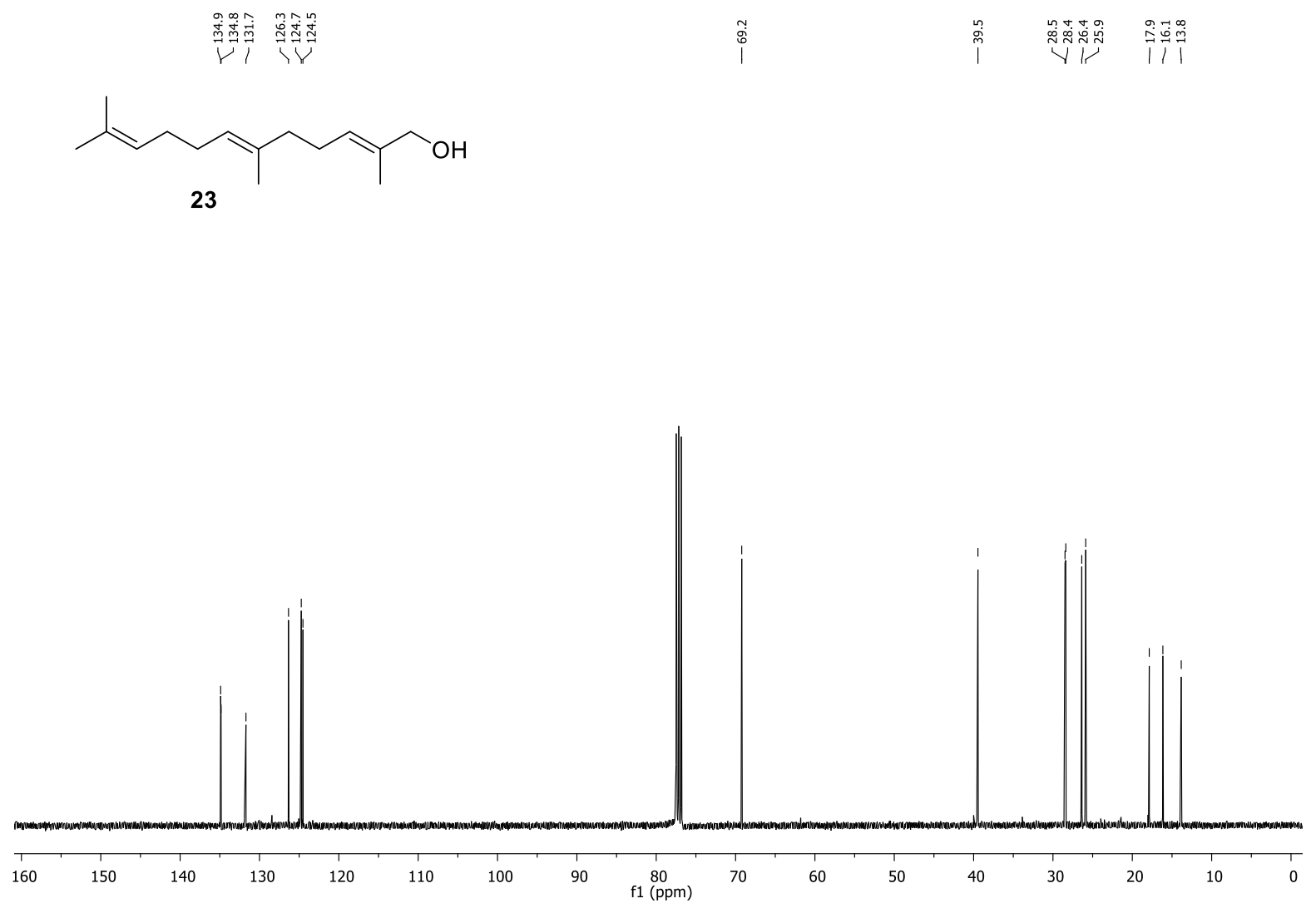
${ }^{1} \mathrm{H}-\mathrm{NMR}\left(400 \mathrm{MHz}, \mathrm{D}_{2} \mathrm{O}\right)$ :
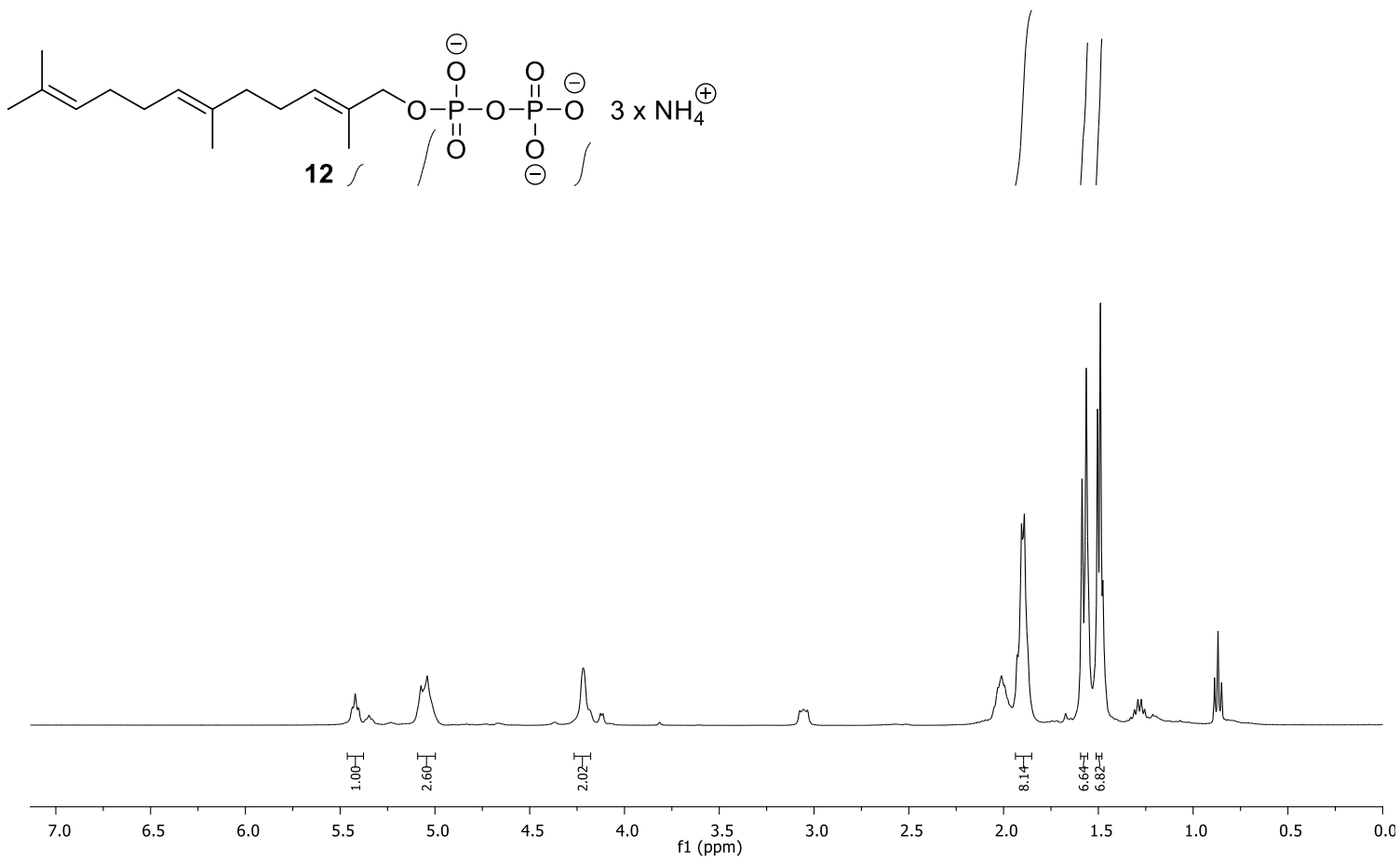

${ }^{13} \mathrm{C}-\mathrm{NMR}\left(100 \mathrm{MHz}, \mathrm{D}_{2} \mathrm{O}\right)$ :

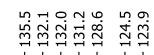

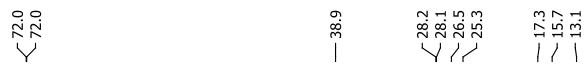<smiles></smiles>

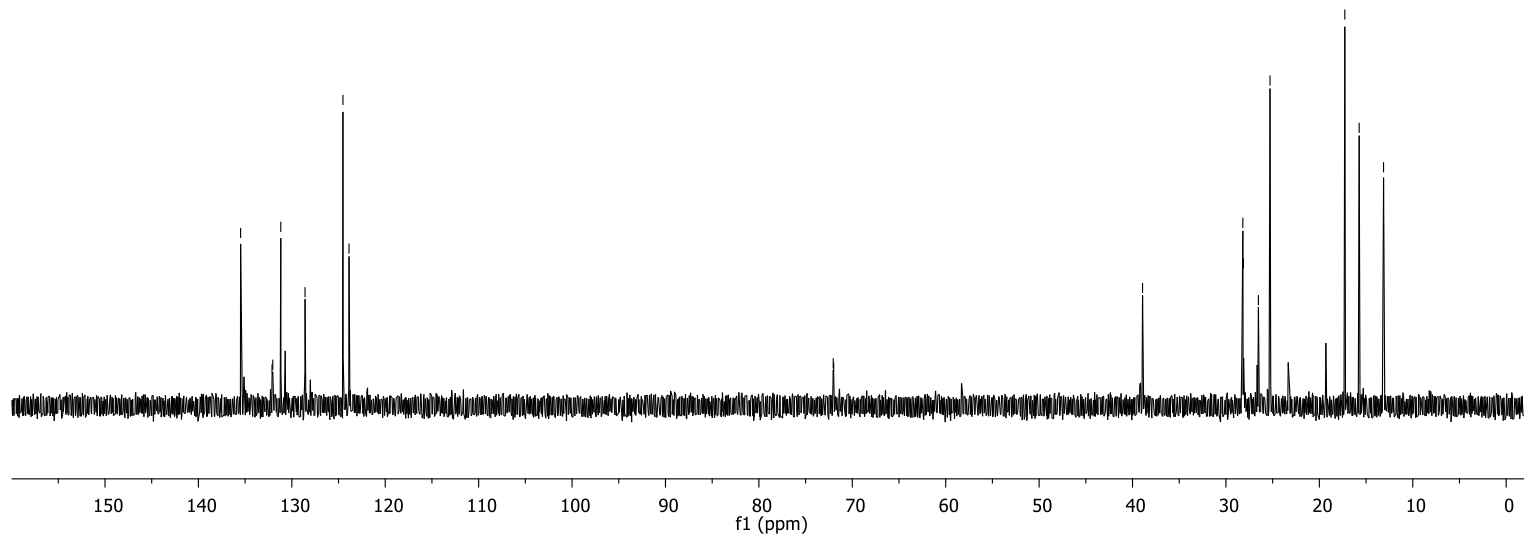

